


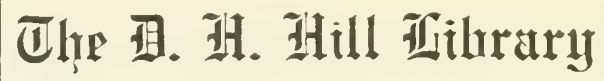
Ainth Caralima state Aluiuersity

Special

Collections

SF 523

D4

v. 1 
THIS BOOK MUST NOT BE TAKEN FROM THE LIBRARY BUILDING.

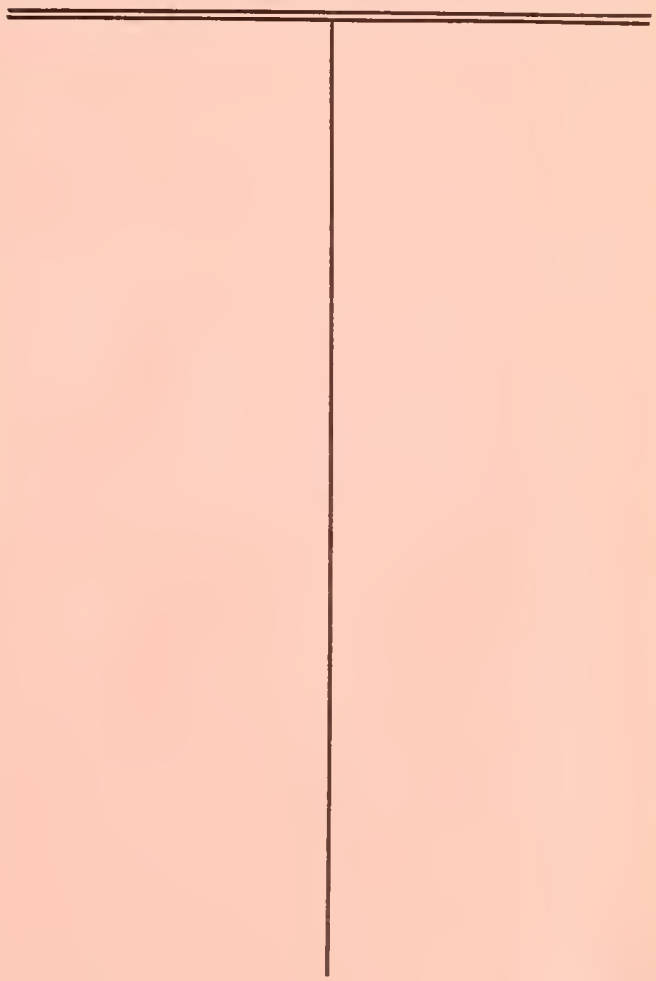


Digitized by the Internet Archive in 2009 with funding from NCSU Libraries 






\section{TRAITE COMPLET}

S U R

\section{LES A B E L LES,}

A V E C

Une Méthode nouvelle de les gouverner, telle qu'elle se pratique à Syra, île de l'Archipel;

Précédé d'un Précis historique et économique de cette île.

$$
\text { DÉDIÉ A M A ME. }
$$

Par M. l'abbé De l a R occ a, Vicaire-Général de Syra.

Admiranda tibi levium spectacula rerum:

Magnanimosque duces, totiusque ex ordine gentis,

Mores et studia, et populos, et prælia dicam.

In tenui labor, at tenuis non gloria..... VIR G: Georg. 4 .

TOME PREMIER.

DE L'IMPRIMERIE DE MONSIEUR.

$$
\text { A P A R I S, }
$$

Chez B L E UET père, Libraire, pont Saint-Michel. 
Torstoment 


\section{A M A D A M E.}

$J_{A I}$ dì à l'intérêt que $M I D A$ A ME daigne prendre à tous les détails de l'économie rurale, principalement à l'entretien des abeilles, l'ordre qui m'a élé donné de construire un nucher, à la mainière des Orientaux, dans ses pittoresques jardins de Montrenil.

Anjourithi, jose esperrer des bontés de MA D A ME, la permission de faire paroître sous ses auspices un ouvrage dans lequel j'ai développé mes principes sur le résrime des abeilles.

Convaincudu vice de la méthode adoptée par les cultivateurs francois, je n'ai pui részster à l'envie de leur faire connoître les avantages des procédés suivis dans le Levant ma patrie, ois cet objet fait une branche de conmerce considerable. 
Des obsereations exactes et des expériences multipliées m'ont mis en élat de composer ce Traité élémentaire, que j'ai tâché de rendre aussi complet qu'il m'a été possible. Heureux si mon travail est un jour de quelque utilité, et si je puis ainsi payer à la Nation françoise la delle que mon allachement et ma reconnoissance m'ont imposée envers elle!

Honoré de la protection de $M A D A M E$, quel succès n'ai-je pas lieu d'attendre pour mon livre! Le public, en le voyant décoréd'un nom qui lui est si cher, en sera sans doute plus disposé à m'accorder son suffrage.

Je suis ayec le plus profond respect,

\section{$M A D A M E_{3}$}

Votre très-humble et trèsobéissant serviteur,

L'abbé Del L A Rocca,

Vicaire-général de l'île de Syra dans l'Archipel. 


\section{A VAN T-P R OPOS.}

Au titre seul d'un Traité sur les abeilles, composépar un Lcvantin, il se trouvera surement quelqu'un qui ne manquera pas d'objecter,

$1^{\circ}$. Qu'il a déja paru un si grand nombre d'ouvrages sur cette natière, qu'il est fastidienx d'en présenter de nouveaux;

$2^{\circ}$. Qu'il n'est guère possible que dans l'Archipel, où règne l'ignorance sur tout ce qui est relatif aux arts, il puisse exister des pratiques agricoles capables d'instruire l'Europe ( sur-tout la France), et assez parfaites pour lui en faire adopter de nouvelles dans cette branche d'économie rurale.

Quant à la première objection, je répondrai que la multiplicité des ouvrages sur cette matière, n'est pas une preuve que le sujet soit épuisé. Ce que les esprits superficiels regardent comme une grande 
opulence, n'est souvent que la preuve d'une disette réelle. Ce raisonnement est confirmé par ce grand nombre de traités sur les abeilles, dont la littérature est inondée. Ils ont presque tous répété les mêmes erreurs, les mêmes préjugés.

J'observerai, quant à la seconde, qu'en cherchant chez les écrivains de l'antiquité, plutôt des comnoissances utiles que curienses, on a vu combien les Grecs aroient excellé dans l'agriculure et daus l'économie domestique. En vain prétendroit - on que leurs lumières sont absolument perdues pour wous, et que leurs pratiques sont tombées en désuétude. Des conquérans peuvent changer la face des empires; mais les arts de première nécessité sont immuables, et les peuples conquis continuent de les cultiver d'après les mêmes principes. Cctattachement pour les anciens usages est. constant dans les îles, à cause du peu de relation de leurs habitans arec les peuples du continent. Celles de l'Archipel, habitées parles 


$$
\text { A } \dot{\mathrm{V}} \mathrm{A} \text { N T-P R O P O } 3 .
$$

Grecs qui se rendirent si célèbres dans tous. les arts à l'époque du règne d'Alexandre, furent subjuguées par les Romains ; mais le peuple conquis conserva ses anciemnes pratiques. Il en fut de même lors de l'invasion par les Turcs; ce qui paroît démontrer que nos principes en économic rurale sont encore, en grande partie, le résultat des réflexions et de l'expérience des plus grands hommes de l'antiquité.

Pénétré de ces vérités, et jaloux de biers. mériter de la Nation francoise et de toute l'Europe, j’ai cru devoir m'élever contre des erreurs préjudiciables à l'agriculture, et indiquer les moyens de s'en garantir ì l'avenir, en présentant une manière nouvelle de soiguer lesabcilles d'après la méthode pratiquée dans l'ile de Syra, me des Cyclades.

Levantin, né à Constantinople, j’ai fait un très-long séjour daus cette même île, où j’ai toujours си un goût singulier pour tout ce qui concerne les travaux de la campagne, et sur-tout pour l'éducatiour des: a is 
abeilles. Je me suis livré à l'étude de ces précieux insectes avec toute la constance imaginable. Je n'ai épargné ni dépenses, ni peines, ni fatigues ; j'y avois rasscmblé un nombre suffisant de ruches, pour me procurer toutes les connoissances dont je faisois la recherche; je voulois savoir élever les abeilles, et les préserver de tous leurs ennemis. Privé de livres dont la lecture auroit pu m'instruire dans cettc partie de l'économie rurale, j’ai consulté les vieillards : leur expérience et mes travaux m'ont mis à portée de perfectionner à Syra l’éducation des abeilles. J'étois livré à ces occupations, lorsque j'ai entrepris mon voyage en France. J'ai passé une seconde fois par l'Italie (1) ; et ce voyage m'a fourni l'occasion d'acquérir de plus grandes lumières. A mon arrivée en France, j'ai ln tous les auteurs qui ont traité cette matière; tels

(1) Dans ma jeunesse, j’avois déja séjournéà Rome, les huit ou neuf années employées à mes études de collegge. 
que MM. de Réaumur, Bonnet de Genève, Ducarnede Blangis, La Grenéc, Pingeron, Duchet, Wildman avec les notes de Contardi, l'Encyclopédie ancienne etmoderne, et quelques autres anteurs francois et italicns. J'ai lu anssi quelques fragmens de M. de Buffon, qui regardent nos insectes.

Après toutes ces lectures, je me suis confirmé dans la persuasion où j’étois déja, que nous avions à Syra, pour la culture des abcilles, une méthode préférable à celles qui sont indiquées par ces différens auteurs.

En effet, de tous les amateurs à qui j'ai fait part de ma facon de penser à ce sujet, et à qui j’ai communiqué quelques-uns de nos principes, il ı'en est pas un qui ne soit de mon sentiment, et qui ne m'ait, en quelque sorte, engagé à faire un traité sur les abeilles. Ils mont tous assuré qu'il seroit favorablement accueilli et fort utile en France, où l'éducation des abeilles est si négligée, et où la cire est si rare. J'ai 
saisi avec empressement cette ocasion de témoigner à la France l'attachement que je lui ai vouée dans tous les momens de ma vie.

Mon intention d'abord n'avoit été que de faire un petit traité, pour exposer ce qui se pratique dans l'ile de Syra, sur l'éducation des abeilles; et pour mieux remplir mon projet, javois la avec la plus grande attention tout ce yưn avoit déja écrit sur cette matière. Le résultat de cette lecture fut qu'il y avoit me infunité de particularités concernant l'histoireéconomicpuc et l'histoire naturelle desabeilles à vérifier, et que tontes ces particularités étoient ou mal entendues on mal appliquées par les auteurs. Insensiblement mon goût pour l'éduration des abeilles, mon amour pour la vérité et mon zèle pour le bien public, mont entrainé plus loin que je ne me l'étois proposé. Je me suis trouvé avoir fait un Traité complet sur les abeilles.

M. Pingeronérrivoit, il y a quelrquesan- 
nées, quaprès les diverses questions qui ont été proposées dans les ouvrages périodiques, quaprès les réponses et les mémoires amxquels ces questions ont domné lieu, on devoit espérer que tous ces matérianx exciterojent le zèle de quelque amateur, et l'engageroicutà composerum Traité complet sur cetiuportant sujet. Lionvrage que je présente aujomrl'hui est donc nécessaireàla sociétéentière, et d'autant plus nécessaire, quil non existe aucun de complet sur cetle matière, quoique nous ayons déja plusieurs productions qui portent le titre de Traités complets : d'ailleurs, ces traités sont imparfaits et défectuenx dans tout ce qui regarde la pratique. Maiş pour ne point être accusé de vouloir trop les déprimer, je vais rapporter à ce sujet le seniment desauteurs les plus modernes.

M. Paltean, cité par M. Ducarne, dans la préface de son Traité sur les abeilles, parle ainsi de tous ceux qui ont écrit avant lui : L'histoire des abeilles, dit-il, 
* et la manière de les gouverner, ont exercé " une infinité d'Ecrivains de toutes les clas" ses et de tous les siècles. Les uns se sont "principalement appliqués à étudier leur * police, à observerleur manœuvre, à suivre " leurs procédés; et parniceux-lá, on peut " compter des anciens, et même quelques " modernes qui ont mêlé beaucoup de fa* bles avec un assez petit nombre de vé"rités. D'autres n'ont presque parlé des " abeilles que relativement à la manière " de les élever et d'en tirer un bon parti. " Si leurs onvrages renferment quelques " traits de l'histoire de ces insectes, ce sont “ des anecdoles romanesques, des prodiges, " des merveilles dont ils ne domment an* cune prenve, et qui n’ont jamais existé * que dans les Géorgiques de Virgile, ou * dans l’imagination séduite de quelques "anciens, dont ces compilateurs ne sout " que les fidèles échos. Ceux-là sont les au" teurs de cette mulitude de petits traités * et de méthodes, dont tout le mérite ne 
* consiste souvent que dans la répétition

* de ce que l'on savoit déja, ou dansl'énu* mération de quelques expériences, de " quelques observations particulières sur * la manière ordinaire de gonverner les * abeilles. Quelques-uns enfin ont heureu* sement réuni ces deux objets, la prati" que et l'histoire, l'usage et la spécula* tion. Des académiciens, aussi habiles ob. * servateurs que citoyens zélés, se sont mis " sur les rangs, et nous ont donné tout ce * qu'on pouvoit espérer de mieux alors; * mais ces auteurs, quelque habiles qu'ils * fussent, n'ayant pu donner à cette étude - tout le temps et toute l'application qu'elle " exige, ont laissé encore à desirer une in" finité de choses, dont un grand nombre " d'expériences répétées plusieurs fois pou" voient les instruire. Ce n'est pas que, sur " l'histoire naturelle de ces insectes, oni " n'ait lieu d'être satisfait. M. de Réaumur - sur-tout, aidé des expériences de ses pré- décesseurs, a presque porté cette partie 
« àsaperfection, autant aumoins que pou" voit le faire un homme qui ne s'y est ap" pliquéque pendant cinq ou six ans; car, " malgré toutes les lumières qu'on trouve " dans son ouvrage, il y manque encore " quelque chose, que peut-être on décou" vrira par la suite. Mais il n'en est pas de “ même des préceptes qu'on nous a don"nés jusqu’ici relativement à la manière " de les conduire, et d'en tirer un bon parti. "On est encore, sur ce demier objet, bien * éloigné de la perfection; et malgré " les louables efforts de tous ccux qui se "sont intéressés au sort de ces insectes, " lacire etle miel n'en sont pas moins chers, * ni les abeilles plus communes. »

Quelque estimable, ajoute M. Ducarne, et quelque bien écrit que soit l'onvrage de M. Palteau, intitulé, Nonselle construction de ruches de bois, on n'en est gruère plus avancé.

"Nous en arous d'antres encore; mais * ce sont, la plupart, des mémoires déta- 
* chés qui ne traitent guère que de quel"que objet particulier. M. de Massac " pourtant, et M. du Houx, ont donné * chacun un traité qui embrasse toute l'éco- nomie de cette instruction; mais ils sont " si abrégés l'un et l'autre, qu'on ne peut " les regarder que comme des espèces de " manuels journaliers. D'ailleurs, l'un et “l'autre n'ayant fait qu'abréger M. Pal" teau, qu'ils ont cru pouvoir suivre, et * leur dessein n'étant guère que de faire " part au public, chacun, d'une nouvelle " construction de ruches, entées sur celles “ de M. Palteau, ces ourrages ne peuvent "satisfaire un homme qui veut des preuves " et du détail, et qui cherche à s'instruire; " sans compter que, dans bien des circons“ tances, leursinstructions ne se trouvent " point toujours d'accord avec l'expérience. - Enfin, pour ce qui regarde l'ouvrage de * M. Pingeron sur l'éducation des abeilles, “ ajoute M. Ducarne, l'auteur de cet ou\& vrage y a rassemblé ce qu'on avoit dit de 
" mieux jusqüici sur les abeilles; mais “ n’étant pas, comme l’on dit, du métier, * il n’a pu faire mieux, et savoir par lui" même ce qüil eût fallu prendre ou re" jeter. Cet ouvragre, quelque abrégé qu’il * soit, m’a paru pourtant le meilleur de " beaucoup de tous cèx qui ont été écrits * jusquaujourd'hui. On est donc encore, * conclut M. Ducarne, réduit à demander " un bon ouvrage sur l'éducation des " abeilles."

Voilà le sentiment de M. Ducarne sur les auteurs qui l'ont précédé, et qui ont traité cette matière.

M. La Grenée, qui a écrit en 1783 , reproche aussi à presque tous les auteurs modernes d'avoir tellement chargé leurs ouvrages de préceptes, ou dispendieux, ou difficiles, ou même impossibles dans la pratique, qu'ils ne sont propres qu’à faire perdre toute envie d'élever des abeilles, à ceux qui en avoient déja formé le projet. Parlant ensuite de l'ourrage de M. Du- 
carne, il dit: " 11 s'en fant de beaucoup que " tous les paysans à yui on voudroit con" seiller d'avoir des abeilles, soient aussi "pénétraus que le persomngge auquel M. "Ducarne adresse ses lecons."

Quelques anteurs Italicns n'en jugent pas différemment, et voici ce qu'en pense M. Contardi. "L'Italic, dit-il, n’a pas eu " jusqu’à nos jours, sur cette partie d'éco" nomie, un ouvrage qui puisse être re"gardé comme élémentaire. Je ne compte " pas ce qu'ont 'écrit Crescentio, Gallo, "Falconi, Tanara, et autres semblables " anteurs, qui tous n'ont traité des abeilles " qu'en passant et accidentellement; car il. " est d'un usage constant que ceux qui "écrivent sur l'agriculture, ne manquent * pas de faire un long chapitre sur l'é" ducation des abeilles. Nous avons vu * deux seuls petits ouvrages sur cette ma* tière; l'un est de Joseph Falchini, Flo* rentin, intitulé : Nouvelle el agréable ins"Iruction sur les abeilles, imprimé à Flow Tome $I$. 
a reuce en 1747 ; l'autre est de Jérome "Maroni, de Vérone, in-4”. imprimé à “Vérone en 1761: mais l'un et l'autre sont " de pauvres ouvrages; on n'y trouve au« cums raisonnemens justes; ils ne sont rem"plis que des fables des anciens qui adop" toient aveuglément tous les préjugés du. “ temps, et ne présentoient danslcursécrits " qu’un faux mervcilleux qui séduisoit les "lecteurs, quoique ricu ne lût plus opposé " auxfaits et à l’expérience. " Je n'en dirai. pas autant des notes de M. Contarlisurl’ouvrage de Wildman; elles sonit judicieuses, et prouvent quil avoit en effet de grandes commoissances, fruit de l'étude et de l'expérience sur l'éducation des abeilles.

Après les éloges que.jagvois entendu faire de Wildman, (c'est-i-dire homme sauvage), je pensois que son ouvrage étoit un chefd'œuvre; mais celui que j'ai lı, traduit en italien par Contardi, m’a bien détrompé. '

La France, l'Italic et l'Angletere n'ayant produit aucun bou ouvrage sur l'éducation 
des abeilles, on ne doit gguère l'attendre des autres parties dumonde. Au surplus,s silexistoit en Europe quelque bon traité sur les abeilles, en quelque langue que ce fît, il auroit indubitablensent été traduit aussitôt en francois.

Ainsi, je puis répéter arec MI. Ducarne: "On est donc encore réduit à demander “ un bon ouvrage surl'éducation des abeil" les, tant pour la France que pour le reste " de l'Europe. "

Quant à celui que je présente au public, ce n'est pas à moi d'en faire l'élogre. Les connoisseurs, après l'avoir lu avec attention, décideront sil est de nature à remplir l'objet que je me suis proposé, et s̈il peut être profitable à la Nation Francoise.

Les auteurs dont jai d'abord fait le plus grand usage, sont MM. Ducame, La Grenée, Contardi, de Bomare, Pingeron et Duchet.Dès quejai eu comoissance de l'ouvrage de ce dernier, j’en ai tiré le meilleur porri possible, ainsi que de l'article abeilles de b) $\mathrm{ij}$ 
M. l'abbé Teissier dansl'Encyclopédie méthodique.J'ai lucet article avec le plus grand plaisir, et il ma beaucoup servi, quoiqu'il fût le demier écrit que j'eusse découvert sur les abeilles.

Puisque la manière d'élever les abeilles, que je présente au public, est d'après la méthode qui se pratique à Syra, île de l'Archipel, on ne trouvera sans doute pas déplacé qu'avant tout je doune un précis de cette même île, et que je lasse comnoître plusieurs autres pratiques économiques et rurales qui y ont lieu.

Je commencerai ce précis par un tableau rapide de tontes les îles de l'Archipel. Dans ce tablean, je parlerai, entr'autres objets, dc la manière dont se formèrent aur temps de la conquête de Constantinople par Jes Francs, le cluché de l'Archipel ou de Naxie, ainsi que plusieurs autres petites sonveranetés qui furent possédées par des scigncurs Francois; et je dirai comment ce mêne duché de Naxie passa sous la domi- 


$$
\Lambda F M T-P R O P O S .
$$

mation des Turcs, avec un très-court détail sur les ducs de Naxic; ensuite je parlerai de l'état ancien et moderue de Syra. et d'un monument astronomique qui marquoit les comrersions du soleil dès le temps d'Homère. Jexposerai la manière dont cette ile est gouvलnée, tant pour ce qui regarde le temporel que le spirituel. On verra aprés la protection que le Roi de France acorde à torts les catholiques de la domination des Thures, et sur-tout aux Syriotes, et la recomnoissance de ceux-ci envers les sujets du Roi. En parlant du caractère et du génic dese Syriotes, je ferai roir que les Grees out toutes les dispositions de leurs ancêtres pour les arts et pour les sciences; de sorte que sils étoient en état de s'y appliquer sérieusement, ils pouroient dérenir ce que les anciens Grecs étoicnt, malgré tout ce que MT. Paw dit sur la dégradatios des Grees modernes, dausses recherches philosophiques sur les Grecs anciens. Je passe 
ensuice à la langue des Syriotes et des Grecs modernes. Je fais roir qu'elle est une vraic langue grecque, qui nous a été transmise par nos ancêtres; de plus, je fais comnottre que la vraie prononciation de celte langue doit être celle qui est en usage chez les Grecs modernes. Après ces détails, je viens à parler de plusieurs pratiques économiques et rurales, qui peuvent être très-intéressantes pour le public, telles que la manière de conserver les bleds, de semer le coton, elc. Je métends sut-tont sur la cappification des figniers, qui est un point qui peut intéresser beaucoup l'histoire naturelle et la botanique.

Limné et quelques autres autcurs ont trailécette matière; mais, n’étant pas sur les lieux on ceute méthode de caprifier les figniers est en usage, plusicurs circonstances très-intéressantes ont été ignorées par eux; ce qui rendoit cette partie d'histoire naturelle moins déreloppée et moins - Gire. Etant né clans lo pays où la 
caprification est muiversellenent pratiquéc, et l’avant noi-mênue cxécutée plusicurs fois, el observée arec aftention, jai fait des découvertes qui répandrout 113 grand joursur tout ce que ces autems ont dil à ce sujet. Eufiu jexpose au public m moyeui simple, mais très-sûr, que nous pratiquons dans le Ierant, ponr guérir la jaunisse la plus invétérée. J'ai fait encore à ce sujet quelques recherches pour voir si ce procédé, ainsi que colui de M. dOr dans la formation de son eau (appelée de son $110 m$ l'eau d'Or, et qui a beaucoup d'aualogie arec notre procédé pour la gutérison de la jaunisse), étoit fondé dans les principes de la chimie et de la médecine; et je soumets au jugement du public, surtout à celui dés persomnes qui par état sont à portée d'en faire une juste appréciation, les décourertes que jecroisaroir faites à cet égard.

J'entre ensuite dans mon Traité sur les abeilles, divisé én 7 livres. Le premier, qui 
comprend la culture des abeilles en général, avec le précis historique de Syra, formera le premicr volume. Le second sera composé du livre denx, qui traite des ruches; du troisième, qui traite des différentes espèces dle mouches qui les peuplent; et du quatrième, consacré entièrement aux essaims.

Le troisième volume sera composé des trois derniers livres; l'un, sur les travaux intérieurs de nos insectes, et sur les différentes matières qu'ils rapportent dans leur ruche ; l'autre, sur leurs ennemis, sur leurs maladies et lesremèdesquileur convienuen!; et le dernier, sur la manière de dégraisser les ruches et de tirer le miel et la cire des rayons.

J'avois presque achevéce 'Traité sur les abeilles, et je ne comnoissois ni les découvertes, ni les expériences des sociétés des abeilles, établics en Lusace, daus le Palatiuat, à Francfort, et daus d’autres villes d'Allemagne. J'ignorois sur-tont la fameuse décourcrie de M. Schirach, dont 


$$
\text { A V A N T - PROPOS. }
$$

je n'avois d'autre commoissance que ce que M. Ducarue nous a dit à ce sujet, et que je rapporte vers la fin du quatrième livre. Je ne connoissois pas non plus les expériences intéressantes de M. Ricms, celles de M. Mattorl, et d'autres auteurs dont je n’avois aucune idée : je savois seulement, d’après Contardi, que ces amateurs avoient travaillé sur l'histoire naturelle des abcilles.

Il m'est enfin tombé dans les mains Ia Contemplation de la Nature, ouvrage de.M. Bonnet, oû se trouvent détaillées, dans cinq mémoires, les recherches de tous ces savaus sur les abcilles; ainsi que cclui de M. Schirach, qui conticut sa fameuse découverte sur la production de la reineabeille.

Malheureusement on en pent dire presque antant de toutes ces découvertes, que de celle de M. Schirach, qui n’a pas été mieux connue de son auteurque de M. Bonnet. La plupart des Naturalistes leur ont 
XYvj

A V A N T-PROPOS.

donné des explications forcées, qui contrarient et révoltent la saine raison, en bouleversant tomles les commoissances consacrées par la main du temps et de l'expérience.

Toila pourquoi je me suis déterminé à domner un quatriène rolume, oì jai inséré la dissertation que M. Schirach a Ine dans la snciété économique de Klein-Bautzen, les cing mémoires de M. Bonnet sur la découverte de M. Schirach, et sur cellesdes autres sociétés économiques d'Allemagne, avec des explications. J'y ai pareillement ajonté la dissertation de $\mathbf{M}$. Haturf, qui contient des recherches physiques pour savoir si la reine-abeille doit. être fécondée par les faux-bourdous.

l'ai accompagné tout cela d'un grand nombre d'observations, tendantes à réfuter lesdifférentes opinions de ces savans. Desexplications claires et simples metront lihistoire natmelle desabeilles dans le jour le plus limineux, et tel que pent-être l'histoire des 
autres insectes en fournit pen d'exemples.

Dans me desobservalions de M. Bommet, quisontà la suite de sesmémoires, on trouve une nouvelle opinion sur l'originc de la cire, entièrement opposéc à celle de M. de Réaumur. M.Duchce, chapelain de Remaufeus, canton de Fribourg en Suisse, a parlé diffusément de cette nouvelle opinion dans son Traité sur les abeilles. Je me stis entpressé de me procurer lonvage de M. Duchet; et jai fait des notes qui détruisent tont son systême. Je dois cependant convenir que sa dissertation contient des recherches trè-curicuses sur les abeilles: il combat arec beancoup rle force l'opinion de M. de Réamur qui soutient que la cire provient de la poussière des étanines, digérée dans l'estomac des abeilles : sentiment que nons réfutons aussi au cinquieme lirre. Après les peines que je me suis domnées pour rendre ces matières éridentes et les appurer des raisomeneus les plus solides, et les plas propres à persuader éà conrain- 
XXviij A VANT-PROPOS:

cre, jai quelque lieu die me flatter que le public recerra favorablement cet ouvage, d'autant plusqu'il pourra y rencontrer quelques idées nourclles, et rapables de piquer sa curiosité ; ainsi que plusieurs anecdotes inconmmes. J'ose espérer quiil ama assez d'indulgence pourme passer quclques fautes de style. J'ai écrit daus une langue qui m'est étrangère, et par couséquent je me suis vu forcé de me servir de trucheman (1). Malheureusement il n'est pas aussi facile qu'on le peuse de s'en procurer de la nature de ceux qui mamoient été nécessaires. La France abonde en gens habiles sans doute; mais il en est pen qui roulussent consacrer icurs momeus à revoir avecsoin un ouvragge Vaussilongue haleme, et guidemandeautant de patience. Dailleurs, il cu est beauroup

(1) L.es Tergimans, (expression turque), d'où les François ont formé leur mot Trucheman, sont des interprètes des François dans les États du Grand-Seigneur. Ill est bien naturel qu’à sor tour un sujet turc ait besoin de trucheman en France. 
A V A N T-P RO POS.

pour qui desouvrages de lanaturedecelui-ci ont peu de charmes: on est donc sonvent obligé de faire usage des persommes que le hasard présente; et ces choix ne sont pas toujours également hemeux. On s'appercevraaisément quemon ouvrage a passé par différentes mains; et qu'il est écrit plus ou moins mal, selon que j’ai été bien ou mal secondé. Une pareille gêne a dî̀ nccessairement produire des répétitions, des redites; je les ai fait disparoître autant que je l'ai pu : mais, malgré tous mes soins, je n’ai pas toujours été le maître de les éviter. Je pric donc le lecteur de vouloir bien me les pardonner, et je me flatte qu'il s'y prêtera d'autant plus volontiers, que j’ai moins eu en vue son amusement que son instruction et son intérêt.

Voilà pourquoi je me suis particulièrement attaché à être clair, persuadé que, sans le mérite de la clarté, les meilleures productions perdent infiniment de leur prix. Pour moi, j’ai été d'autant plus sur la dé- 
$\mathrm{XXX}$ A V A N T - P R O P O S.

fensive à cet égard, que je sentois à merveille que çanroit été manqquer totalement mon but, que de ne pas ne mettre à portée d'être cutendu par toutes sortes de personncs. En conséquence jai cherché, autant que je l'ai pu, à racheter par lá mes autres défauts. C'est au public à juger si le succès a courommé mes efforis.

On pourra me reprocher d'aroir fait un onvrage trop voluminenx, et pent-être diffus. Je répondrai àcela, que je domne me méthode de gonverner les abeillcs, entièrement nonvelle. Il falloit combattre les ancicunes pratiques, faire voir plusieurs de leurs incouvénicus, lesmettre souvent cucomparaison avec nos procédés, et démontrer l'arantage de ceux-ci sur les anciennes : outre que dans l'histoire naturelle des abeilles, jai fait des corrections et des additions importantes qui jettent un jour tout nouveau sur cette partic intéressante. Tout cela exige beaucoup de mots, et les mots forment des rolunes. 
Nais, dira-t-ou peut-être encore, de semblables ouvrages ne doivent se faire que pour les gens de la campagne; par couséquent, ils doivent être courts et d'un prix modique..... Je ne crois pas que la plupart de ceux qu'on appelle paysans (1), s'instruisent avec des livres. Dans tous les pays ci-

(1) "Le paysan françois, qu’il s'agit d'instruire des nouvelles déconvertes en agriculture, ne lit point or presque point : on doit donc le compter pour rien dans l'usage qu'on peut faire des mémoires des compagnies savautes. Accoutumé, dès l'enfance, à une pratique qu'il tient de ses pères, il n'en connoît et n'en veut pas connoitre une autre, à moins que sous ses yeux, il n'en voie les bons effets; c'est le langage de l'expérience qu'il faut lui parler. Que le hasard place dans chaque province, dans chaque canton, un homme intelligent, ami de l'agriculture, patient, et capable d'inspirer de la confiance à tout ce qui l'environne, qu'il y fasse des expériences en s'associant pour cela des laboureurs, et qu'il les metie en état de juger enx-mêmes des résultats; sans efforts pour les convaincre, sans livres, sans encouragemens même, il les verra lentement à la vérité, adopter les méthocies nouvelles qui auront eu des succès, et dont ils se croiront les inventeurs, parce qu'ils auront coopéré aux essais qu'on aura 
xxxij A V A N T - P R O POS.

vilisés, ce sont les curés, les fermiers; et autres citoyens instruits, qui peuvent communiquer leurs idées et leurs comnoissances à leurs paroissiens et à leurs voisius.

faits. C'est ainsi, et non autrement, que les connoissances dissiperont peu-à-peu les ténébres de l'ignorance et des préjugés repandus sur l'agriculture. "Encyclopédie méthodique. Agriculture. Discours préliminaire, par M. l'abbé Tessier, pag. 36.

\section{A V I S.}

LA planche qui suit représente la ville et le port de Syra, vas du eûté de la terre, avec les trois sortes de secours que ses habitans donnent anx vaisseaux françois, daus les temps cle naufiage, d'attaque des corsaires; ou de contagion.

Elle a été gravéc, il y a quelques anuées, pour être dédicie et présentée an Roi : mais l'ayant jugée trop petite, $j$ 'en ai fait graver unc plus grande, dont la vue cst du cûté de la mer, et remplit mieux mon objet.

J'ii cru que les amateurs qui voudront se procurer mon ourrage, ne me sauroient pas mauvais gré d'aroir placé à la tête cette gravure din Précis historique de l’̂̀le de S.yra. 


\section{$P$ R É C I S}

HISTORIQUE ET ÉCONOMIQUE SUR L'ILE DE SYRA.

$L_{A}$ manière de gouverner les abeilles, que je présente au public, n'étant à-peuprès que celle qu'on pratique à Syra, quelques personnes, dont je respecte l'opinion, m'ont engagé à met tre à la tête de mon ouvrage un précis historique de cette île. Je m'y suis déterminé d'autant plus volontiers, que ce sera encore pour moi l'occasion d'indiquer quelques procédés particuliers, dont la connoissance ne peut être qu’avantageuse et agréable. Je me propose donc de parler, après avoir exposé un tableau rapide des îles de l'Archipel, $1^{\circ}$. de l'état ancien de Syra; $2^{\circ}$. du philosophe Phérécyde, et du monument astronomique que l'on y royoit Tome $I$. 
dans les temps d'Homère; $3^{\circ}$. de l'état actuel de cette île; $4^{\circ}$. du gouvernement temporel et spirituel de Syra; $5^{\circ}$. de la protection de la France envers ses habitans $; 6^{\circ}$. du caractère et du génie des Syriotes; $7^{\circ}$. D'une courte réfutation de M. Paw, sur ce qu'il dit des Grecs modernes; $8^{\circ}$. de la langue des Syriotes; $9^{\circ}$. de quelques procédés relatifs à l'agriculture, et suivis à Syra; $10^{\circ}$. de la méthode de caprifier le figuier, usitée dans l'île et dans toute la Grèce, depuis les tempsles plus reculés; $11^{\circ}$. enfin, d'un moyen simple et facile dont se servent les Syriotes pour guérir la jaunisse. 


\section{H A P ITR E PR EMIE.R.}

Tableau rapide des iles de l'Archipel (1), d'après l'hislorien des ducs de Vaxie.

Tou tes les îles de l'Archipel sont comprises entre le $35^{\mathrm{e}}$. et le $38^{\mathrm{e}}$. degré. L'air y est extrêmement doux : on n'y connoît presque point

(1) On donnoit anciennement différens noms à cette partie de la Méditerranée qui sépare la Grece européenne de celle de l'Asie, et qui contient toutes ces îles : les principaux sont l'Archipel, la mer Blanche et la mer Égée. Le premier vient du mot 'ágx̀̀, archès coǹmencement, et de wína To, pelago, mer, comme sion disoi t le commencement de la me:", ou le prince des mers. On l'appelle mer Blanche, pour la distinguer de la mer Noire. Les Grees et les Tures ne la nomment pas autrement. Les auteurs varient sur l'origine du mot Egée : l'opinion commune tire celte denomination du golfe Eghena, dans lequel se troure une ile qui porte ce nom.

Les îles grandes et petites qui sont contenues dans cet espace de mer, vont à soixante ou environ, et il y en a très-peu qui ne soient habitees.

\section{H. HILL LIBRARY \\ North Carolina State College}


d'hiver ( I), et c'est une espèce de prodige quand il y gèle. Les chaleurs pourtant n'y sont pas incommodes comme ailleurs. Pendant une grandepartie de l'année, il y règne un vent du nord fort agréable, qui rafraîchit l'air. La mer y contritue aussi à tempérer les ardeurs du grand soleil, qui, sans cela, ne seroient pas supportables (2). Cette température de climat fait que les arbres sont toujours verds, et que

(1) Effectivement à peine les hivers durent-ils trois mois dans tout l'Archipel : cependant le froid y est extrêmement piquant avec les vents du nord, et il faut sy habiller, pendant ce temps-là, au moins aussi chaudement qu'à Paris. J'ai demeuré environ dix-sept ans daus l'Archipel, let je n'y ai pas ru de gelées; nos vieillards même ne les jamais connues : cependant les vents du nord y sont quelquefois si forts, si secs et si froids, qu'ils crispent toute la surface de la terre, et que l'on n'y voit pas un brin d'herbe.

(2) Le soleil y est extrêmement ardent ; cependant lorsque les vents du nord commencent à souffler, on se promène à découvert dans les campagnes, sans se ressentir d'une trop grande chaleur. Il semble qu'Hippocrate, ct $\mathrm{M}$. Paw après lui, attribuoient à ce frappant contraste de saisons, la supériorité du génie des Grecs sur toutes les aulres nations. Voyez lechapitre sur le gézile ct le cuructère des Syriotes. 
SUR L'Ile de Syra. Chap. I.

quelques-uns ont des fleurs presque toute l'année. Les corps y sont sains et robustes; on ne sait ce que c'est que la goutte et la gravelle. La peste, qui ravaye si souvent Constantinople et d'autres pays du Levant, n'approche jamais desîles, à moinsque quelques pestiférés ne l'y apportent daillcurs.

C'est dans l'Archipel qu'il faut aller voir le printemps dans toute sa beauté : cette saison si riante et si fort vantée en Italie, est encore tout autre chose clans les îles, et dans quelques-unes entre autres, coínme à Naxie et à Andros, où il y a quantité de fontaines qui arrosent la te:re. Les orangers et les citronniers parfument l'air par la quantité prodigieuse des fleurs dont ils sont chargés, et qui s'épanouissent aux' premières chaleurs. Les campagnes et les collines sont rouges d'anémones (1). Les

(3) Outre ces fleurs que l'auteur nomme anémones, il y a dans toutes les îles, er sur-tout à Syra, une espèce de lis, appelé ici asphodèle, dont toutes nos collines sont couvertes. Elle s'élève environ de deux à trois pieds, et forme un très-gros bouquet. Cet asphocicle commence à fleurir aı mois de février, et dure encore pendant le mois d'avril. Les collines qui en sont couvertes forment un coup-d'œil dont on ne peut décrire la beauté. 
inontagnes sont couvertes de thym et de lavande. Les abeilles, qui v volent par nuées, en tirent un miel qui est aussi transparent que notre gelée. Les lauriers-roses, que l'on conserve en France arec tant de soin, viennent à l'aventure dans les prairies, et le longr des ruisseaux qui en sont bordés. Rien n'est plus agréable que de voir ces beaux arbres, de la hatuteur de douze à quinze pieds, variés de fleurs rouges ct blanches, se croiser par les branches d'en haut, sur un ruisseau ou sur le lit d'une fontaine, et faire un berceau qui dure quelpuctois un grand quart de liene.

Le terroir y est si bon, et les arbres y viennent si vîte, que jai vu à Naxie des pepius d'orange de Portugal ponsser en moins de huit ans de grands orangers, dont les firuits étoient les plus délicieur du monde, et la tige de l'arbre si haute, quil falloit une longue échelle pour y monter, On pent juger si les vins y sont exquis, et si les anciens enrent raison d'appeler. Naxie lîle de Bacchus. Les raisins y sont mons. tueux, et il arrive sourent que dans un repas, on n'en seri qu'un seul pour le fruit; maisanesi couvre-t-il tone la profonders d'un grand bas$\sin$ : les grains en sont gros comme nos damas 
SUR L'ILE DE SYRA. CHAP. I.

noirs. Il y a dans les îles des raisins de plus de vingt sortes : les muscats de Ténédos et de Samos l'emportent sur tous les autres; ceux de Ténédos sont plus ambrés; ceux de Samos, plus délicats. Les Sentorinois, pour donner une saveur plus exquise à leurs raisins, leur tordent la queue lorsqu'ils commencent à mûrir ; après quelques jours d'un soleil arclent, les raisins deviennent à demi flétris, ce qui fait un vin dont ceux de la Cicutat et de Saint-Laurent n'approchent pas. Les autres sortes de raisins sont l'ä̈dhoni, petit raisin blanc qu'on mange vers la mi-juillet; le samia, gros raisin blanc qu'on fait sécher; le siriqui, ainsi nommé parce qu'il a le goût de la cerise; l'cetonychi, qui a la figure de l'ongle; d'un aigle, et qui est trèssavoureux; le malvoisie, le muscat violet, le corinthe, et plusieurs autres dont les noms me sont échappés.

L'Archipel est le pays des excellentes figues de toutes les sortes; plusieurs espèces de celles que nous cultivons en France, ne servent qu’à nourrir les pourceaux. Naxie et Andros sont renommées pour la grosseur et la bonté de leurs grenades (1), de leurs limonset de leurs cédrats;

(1) J'ai été plusieurs fois à Navie ; je n'y ai jamais ru 
ces derniers que les Grecs appellent kitra, et qui sont une espèce de gros citrons doux (1), croissent sur un arbre à-peu-près de la figure des grands buissons de nos potagers : le fruit en est gros comme la tête; son écorce, que l'on confit au miel, et qui est souveraine contre les maux d'estomac, est rude et fort inégale; le dedans en est d'un goût merveilleux, sur-tout quand les premiers froids du pays ont passé dessus. Les melons et les melons d'eau se sèment par-tout en plein champ comme le blé.

Les oliviers viennent d'une grosscur prodigieuse presque dans tontes les îles; et à Naxie, ils y sont par forêts. La récolte en est si abondante, que cette île seule fournit des huiles à toutes les autres; il n'y en a peut-être point de meilleures dans tout le Levant, ni de plus blanches, ni de plus transparentes.

de ces grenades, ni de eclles d'Andros : je sais que Smyrne estrenommée pour en avoir de belles. C'est à Naxie que se trouvent les beaux cédrats; $j$ 'y en ai vu de deux fois plus gros quc la tête, et qui pèsent 8 à 10 livres.

(1) Je ne sais pas pourquoi notre auteur dit que les cédrals sont doux; leur jus au contraire est anssi acide que celui des citrons ordinaires. 
SUR L'Ile d Syra. Chap. I.

Le climat des îles est admirable pour le gibier ; on ne sauroit croire en quelle abondance il s'y multiplie. A Náxie, où j’en ai vu plus qu'ailleurs, les bois fourmillent de lapins auxquels le serpolet et le thym donnent un fumet très-agréable: on voit courir les lièvres par handes. En autonne les bec-figues, qui sont très-gros et d'un goût délicieux, voltigent par-tont antour des figuiers et des raisins.Les perdrix y sont très-communes, ainsi que les bécasses. Sur les côtes il y a des quantités prodigrieuses de pigreons sauvages, auxquels les aigles (qui sont si puissans qu'ils enlèvent quelquefois de petits veaux dans les pâturages), les milans, les faucons et les autres oiseaux de proie font une guerre continuelle, sans que pour cela ils paroissent diminuer. Dans certains temps de l'aniée, il arrive dans les îles, sur-tuut à Sentorin et à Tine, une surprenante quantité de cailles et de tourterelíes très-yrasses, dont toutes les campagnes sont remplies, sur-tout des premieres. Les habitans en font leur provision pour une grande partie de l'année.

Presque toutes les îles ont quelque chose de particulier. Outre ce que j'en ai dit, Syra est fameuse pour ses toiles de coton; Zéa pour 
son gland, dont les teinturiers de Venise font un grand trafic; Sichino pour son froment, le plus beau de la contrée. Antiparos a des pierres d'aimant : on a trouvé depuis peuà Milo, des émeraudes brutes; ce qui fait juger qu'il y en a un fonds quelque part. Dans Siphanto on a découvert aussi, depuis quelques années, des mines d'argent et decuivre, qui enrichiroient un prince gai voudroit y faire travailler. On tient quil y a aussi des mines d'or à Naxie, du côté du midi; je sais au moins qu'il y a d'excellent émeril, quion vient enlever pour Marseille et pour Venise.

Tous les peuples de l'Archipel sont chrétiens; mais tous ne sont pas catholiques. Le's Latins n'en font tout au plus que la huitieme partic, encore sont-ils répandus en différentes îles, où dansquelques-unes il n'y a qu'un vicaire entretenu par le Saint-Sicge; d'antres, comme Naxie, Syra, Tine, Santorin, ont leurs évêques latins qui les grouvernent. L'archevêque de Naxie est le métropulitain, et cetto église est la seule qui ait retenu son ancien chapitre.

Communément parlant, tous les Grees ont un excellent fonds d'esprit, la conception subtile, les pensées vives, et je n’en ai presque 
point vu qui ne fussent naturellement éloquens: quand ils le veulent, ils sont plaisans, bouffons et comédiens; ils excellent sur-tout dans la pantomime. La règle établie, que la foi ch Grec est nulle, n'est pourtant pas universelle pour tous les Grecs; jen ai connu plusieurs dans l'Archipel, d'une probité, d'une bonne-foi ct d'une sagesse rares, et qui, avec toutes les qualités de leur nation, n'en avoient point les défauts. Je dois même ce témoignage aux Grecs des îles, qu'en grénéral î́s sont plus gुens de bien et plus sincères que ceux de terre-ferme( I ). Tous les Grecs des illes, et sur-tout les femmes, ont une passion excessive pour la danse. La veille des fêtes un peu solennelles, ou d'un patron de quelque église de l'île, on les voit tous arriver par bandes cles lieux les plus éloi gués, et danser toute la muit sur la gorande place de l'église, et dans le jour à l'ombre de quel-

(I) Je crois que cela peut arriver de ce que les Grecs des îles ne fréquentent guère diutres nations qui habitent le continent: ils ont au contraire beaucoup d'occasions de communiquer ave les nations civilistes de l'Europe, qui fréquentent leur wer; et ils riennent eux-mêmes fairc tous lés ans des voyages à Tenisc, à Ancône, à Lirourne, à Trieste et ailleurs. 

ques arbres. Les dames plus importantes, qui peuvent sortir ces jours-là, y viennent à cheval, précédées de musettes ct de tambours, qui annoncent de loin leur arrivée.

De tout ce qui a été dit jusqu'à présent, on pent conclure quil y a peu de pays au monde qui fournissent avec plus de profusion que les Śles de l'Archipel, tout ce qu'il faut pour les besoins et les agrémens de la vic. Et si l'on s'appliquoit à y cultiver le sucre, le café, l'indigo et autres productions de l'Amérique, ce seroit une sceonde terre promise. Après les essais qui ont été laits par le feu comte de Carbury à Céphalonie, île qui est plus au nord que la plupart de celles de l'Archipel, et qui ont eu le plus grand succès, ces plantes du nouveau continent y réussiroient admirablement.

Presque toutes ces îles, grrandes ou petites, eurent ancienncment leurs rois ou tyrans, comme le discnt Homère et d'autres auteurs. Dans le temps ensuite des républiques de la Grèce, la plupart de ces îles, seules, ou plusieurs ensemble, formèrent autant depetites républiques, qui subirent le sort de toutes celles qu'Alexandre le Grand fit passer sous sa domination. Subjuguées par les Romains, soumises à Constan- 
SUR L'ILE DE SyRA. Cha P. I. I3 tinople ou au bas-Empire, après la prise de cette ville par les François et les Vénitiens, il s'en forma, et de presque toute la Grèce, plusieurs petites souverainetés que les seigneurs françois et vénitiens se partagèrent. C'est ce que l'on voit dans Tournefort, qui n'en parle que d'après plusieurs auteurs qu'il ne manque pas de citer.

Voici comment l'auteur de l'histoire des Ducs de l'Archipel nous expose ce fait. La conquête que les Francois firent de l'Empire Grec, l'an mil deux cent quatre, a donné lieu à l'établissement de la souveraineté desducs de l'Archipe!.

Après la prise de Constantinople, Baudouin, comte de Flandre, ayant été élu Empereur avec l'applandissement de l'armée victorieuse, plusieurs seigneurs grecs voulant profiter de la confusion où se trouvoit alor's tout cet empire, s'érigèerent en souverains, sans que Baudouin, encore trop foible dans sa nouvelle conquête, pût s'opposer à ces invasions. Quelques-uns se jetèrent sur les côtes de la mer Egée, où ils se fortifièrent; et quelques autres dans les îles de l'Archipel, d'où ils faisoient des courses continuelles sur les Latins, dont ils ne pouvoient supporter la domination. 
Ce désordre dura autant que le règne de Baudouin : mais Henri son frère ne lui cut pas plutôt succédé, que ce nonvel empereur forma le dessein de détruire tous ces petits souverains. Le moyen dont il se servit pour y réussir, fut de permettre aux principaux seigneurs de sa cour, qu'il vouloit d'ailleurs récompenser pour les grands services quil en avoit reçus, d'armer contre ces rebelles, et de leuri abandonner toutes les conquêtes qu'ils pourroient faire. Le comte de Blois s'empara d'une partie de la Bithynic; le seigneur de Champlit conquit le Péloponèse; Othon de la Roche, gentilhomme bourguignon, jeta ses vues sur Alhènes, dont il se rendit maître, de même que de la ville de Thèbes; Jacques d'Avènes et Ravin Carcerio prirent Négrepont. Et c'est ainsi que les François formèrent divers petits états dans la Grèce, où ils se maintinrent long-temps sous la protection de l'empereur.

Les Vénitiens, qui avoient assisté les François à la prise de Constantinople, et qui avoient eu en partage la 'Thessalie avec une partie de la Macédoine, ne s'apperçurent pas plutôt des grandes conquêtes que les seigneurs François faisvient chaque jour dans la Grèce, qu'ils 
SUR L'IlE DE SyR A. ChaP. I. I 5 voulurent aussi étendre plus loin les bornes de leurs états. Ils permirent donc aux plus considérables d'entre eux d'équiper des vaisseaux; et à l'exemple de l'empereur, ils leur donnèrent aussi toutes les conquêtes qu'ils pourroient faire. Plusieurs mirent en mer : Marc Dandolo, surprit Gallipoli; André Gizi se rendit maître des îles de Tines, de Micone, de Shiro et de Scopelo. Marc Sanudo, lun des capitaines les plus accomplis qu'eût alors la république, ne fut pas moins heureux que les autres : c'est celui-là même qui engagea Boniface, Marquis de Montferrat, à faire échange de l'île de Candie qu'il avoit eu en partage, avec le royaume de Thessalie, qui étoit soumis aux Vénitiens. Ce brave Sanudo tourna ses armes contre l'île de Naxie, et s'en rendit maître avec beaucoup de facilité, ainsi que de Paros, Antiparos, Milo, l'Argentière, Sifanto, Policandro, Nanfio, Nio, et Santorin. L'empereur Henri érigea Naxie en duché, et donna à Sanudu le titre de duc de l'Archipel et de prince de l'Empire. Toutes ces îles soumises à la domination des ducs de Naxie, s'appelloienten Grec Dhucanisa, on les appelle encore aujourd'hui par corruption Dhodhecanisa, les douze îles. 
Les ducs régnèrent dans l'Archipel pendant plus de 300 ans. Il y en eut vingt-un, la moitié presque de la maison de Sanudo, et l'autre moitié de la maison Crispo. En voici les noms.

Marc Sanudo, premier duc. Ce prince avoit d'excellentes qualités, parmi d'autres qui ne l'étoient guère. Il étoit prudent, courageux; mais il se laissa trop emporter à son ambition et à la passion de s'agrandir. Au reste il étoit d'un tempéramment robuste, d'une taille majestueuse, d'un esprit vif et pénétrant, magnifique, bienfaisant, libéral envers ses sujets. Il mourut à l'âge de soixante-sept ans, après avoir gouverné son état fort heureusement près de douzs ans.

Ange Sanudo, second duc, étoit un prince courageux, entreprenant, et qui ne cherchoit que les occasions de se signaler. Ce prince eut toutes les grandes qualités du duc son père, sans en avoir les défauts. Il cessa de vivre à quarante-sept ans.

Marc Sanudo, second du nom, troisième duc. La plupart des historiens confondent ce duc avec Marc Sanudo premier duc, sans faire mention d'Ange Sauudo, dont nous venons de parler. 
SUR L'Iléde Syra. ChaP. I.

C'est ce qui fait qu'un des plus illustres historiens de ces temps, au lieu de six ducs de cette famille, n'en met que quatre dans son histoire bizantine. Ce dac monrut à Milo. Les Latins perdirent infiniment a la mont de ce prince, dans les circonstances fàchenses ou la prise de Constantinople sur les Francois par Michel Paléologne, empereur des Grecs, les avoit mis tons. Il étoit leur conseil et lemr appui. La douleur publique fit son élogre, et si l'on cun croit les mémoires de ces temps-la, il s'est vu peu de souverains plus aimés, plus : espectés et plus regrettés que lui.

Guillaume, quatrième duc, succéda à son père à l'âge de vingt ans. C'étoit un prince plein d'honneur et de probité, à qui la perficlie taisoit horrenr: il combalcoit en brave, et il se fit tonjours un point dhonneur de hasarder tout pour ses amis malheureux.

Nicolas Sanudo, cinquieme duc, fut élevé dès son bas-âge, à tous les exercices militaires. Aucun duc de Naxie n'avoit encore eu une si haute réputation de valeur. Il étuit ambitieux de giloire, et avec cela, d'une droiture et d'une bonne foi généralcment reconnues de tout Tome 1. 
ic monde, bon ami, allić fidele jusqu'au péril de sa vie.

Jean Sanurlo, sixième duc. Sa vie u’a été illustrée par aucun exploit de gaerre considérable; c'ćtrit un bon prince, extrêmement doux, et d'une humeur fort paisible.

Jean Carcerio ou Dalle Careri, septieme duc, du chef de sa femme Sanudo, fille unique du dernier duc, descendoit d'une ancienne famille originaire de Vérone. Ce duc, après sa mort, ne laissa qu'un fils nomné Nicolas, qui n'étoit pas en âge de lui succéder. Sa femme Florence Sanudo, qui étoit encore assez jeune, épousa ei secondes noces le brave Nicolas Samudo, surnommé Spessa banda.

Nicolas Sanudo, second du nom, huitième duc, étoit petit-fils de Marc Sanudo, fière puiné de Guillanme, quatriène duc de Naxie. C'est de ce duc, dont Marin Sanudo, noble Vénitien, parle dans ses lettres, oì il en donne l'idée d'un des plus errands hommes de son temps. Son courage et son intrépidité le firent surnommerSpezá banda, comme qui diroit, celui qui met tout en pièces. Il étoit la terreur des ennemis du nom chréticn, le héros de la Grèce, et ma des pius zólós délenseurs de la religion. 
SUR L'Ille de Syra. Chap. I.

Nicolas Carccrio, fils de Jean Carcerio, succéda aux ćtats de l'A rchipel, qui luiappartcnoient de droit. Ce fut le newvième duc, d'une prudence et d'une valeur au-dessus peut-être d'aucun de ses prédécessenrs. Il fut assassiné par François Crispo, quï lui succéda.

François Crispo, dixième duc, descendoit d'une maison fort ancienne et fort illustre, puisqu'il comptoit des empereurs grrecs parni ses ancêtres; mais ses crimes, et sur-tout sa perficlie à l'égard du duc Carcerio, l'ont clégradé pour jamais dans l'esprit des gens de bien, et sa haute naissance, "n'a servi qu’à le rendre un illustre scélérat.

Jacques Crispo, onziéme duc : ce prince avoit de la valeur et de la prudence; Jean son frère lui succéda.

Jean Crispo, douzième duc, fut d'une complexion délicate, et il ne manquoit ni de valeur, ni d'expérience, ni mème de probité et de bonnefoi, vertus, dit l'auteur de l'historre de tous ces ducs, dont on se piquoit assez peu dans sa famille.

Jacques Crispo, second du nom, tréeizième duc. Ce fut sous ce prince que Constantinople fut pris par les Turcs. Il mourut de phthisie Ia $\mathrm{Bij}$ 
prenièe amnéc de son mariage, laissant ha Duchesse sil femme, grosse.

Jean-Jacques Crispo, quatorzieme due, monnt à treize mois, et par sa mort, laisea lélal dans. le dernier désordre.

Guillamme Cripso, quinziome due, fròe de Jean Crispo, douzieme duc. Le dac Guillanme, n'ayant point d'enfans, nomma à sa mort, le seigneur de Santorin, son nereu, pour successeur au duché, selon leur ancien accord.

Francois Crispo, seizicme duc. Il aroit toujours fait la gुuerre pour la république de Venise; il ne manquoit pas de mérite; mais toules ses actions furcut confondues arec celles des grénélaux Vénitiens.

jacquas Crispo, troisième dn nom, dix-septieme duc, eut pour femme une filie de David Comnène, empereur de Trébizonde

Il neut que deux filles, dont la premièe fut mariée à Dominique Pisani, de la noble famille vénitienne de ce nom.

Jean Crispo, dix-huitieme duc, fière du précédent, qui l'aroit nommé son successcur au préjudice de sa propre fille mariće à Pisani, n'a eu quin fils qui lai surcéda.

Franois Crispo, dix-nenpiome duc. Ce prince, 
иni avec les T'énitiens, fit de grrandes acious militaires dans les grumes contic les 'Tu'ues. Il laissa un fils hóritier de ses états.

Jean Crispo, vingtieme duc. Sous ce prince; le fameux Barberomse fit une descente dans l'île de Naxie, quil siccagea. Le duc laissa le duché à sou fils Jacques Crispo, vingt-unieme et dernier duc de l'Aichipel, quir fut ensuite subjugué par les Tures.

La maniere dont cesévènemens se sont passés, n'etant pas assezconnue dn public, je crois luifaire plaisir de lui en domner nue courte exposition.

Le Duché de Naxie se trouvoit alors, (sous. Jacquesson dernier Duc), dans un déphorable état. Depuis la descente de Barberousse, yui avoit dévasté l’île de Naxie sous le duc jórécédent, et qui avoit rendu tributaire du grand-seigneur le même duc, de 6000 écus d'or par an; depuis ce temps-lì, dis-je, les Crecs ne voulurent plas niobéu, ni contribuer aux dépenses publiques. Le Duc, étoit saus argenent, sans vaisseaux, et selou la destinée ordinaire des malhcareux, sans ajpui. Mais quand il auroit eu tout cela, on pent dire que de l'humeur dont il éloit, jl wien auroit pas moins avaucé sa rune et celle de toute sa maison. Le dangere qui réveille el qui inquiver 
les autres, sembloit comme l'avoir assoupi : il ne sonģ⿻i口 uniquement qu'à ses plaisirs, et pour avoir de quoi y fournir, il n'y eut point de violences auxquelles il ne se portat. Les nobles, qui composoient sa pelite cour, nétoient pas en cela plus sigges que lui; on eût dit quils vouloient toms insulter a sa mauraise fortune; et quese sentant sur le bord du précipice, its se hàtoient demettre ì profit pour leurs plaisirs le peu de templs qui leur restoit. Ce n’étoit dans toute lîle de Naxie, yue débanches et dissolutions continulles. Ces scandales avoient même passé jusquaux gens d'église: le Duc souffroit quils vécusscont daus un dósolude qui fait horreur (1): aussi la colère de Dien ne tarda-telle pas à éclater sur lindigne sonverab qui clomnoit lien à ces abomiations.

Les Grees, ravis de trouver dans les vexations de leur duc, et dans les désordres des Latins,

(1) Ois voit par là qu'il est toujours vrai que la corruption gyenérale des mœurs, est l'avant-coureur de la perte des étais. On ne peut la prérenir et les en sauver, qu'en rappelant les bonnes-monrs, et en récompensant Ja verin. 
SUR L'ILE DE SYR A. Chap. I. 23 de quoi autoriser la hâ̂ne furicuse qui les excitoit toujours contre enx , formerent sourdement le projet de changer de maître; et les choses allèrent si loin, qu'enfin, après plusieurs délibélations secrètes, ils cuvoyèrent denx députés à la porte, pour se plaindre des violences de Jacques Cripyo, ct demander än zrand-seignenir un duc qui litu plus digne de les commander. Le départ des députés et leurs desseins ne purent êtresiserents, que Crispo n'en ent comnoissance : il crut devoir aller humême après enx à Constuntinople ; et comme il nignoroit pas qu'à la Porte tout se faisuit à force d'argent, il eut soin de porter avec lui donze mille écus, sur lesquelsil comptoit extrênement. Mais les députés de Nasie étoicnt déja écoutés, et sa perte étoit résolue. A peine fut-il arrivé, que sans avoir égard à la dignité de sa personne, il fut dépouillé de tous ses biens, et jeté en prison. Il y demenra cinq ou six mois, et n'cn put sortir quà la sollicitation de ses sujets, qui avoient appris que Sélim II, siccesseur de Soliman, vouloit leur donner un Juif pour naitre. Ils mirent tout car usage pour l'empêcher et obtenir le rétablissement le Crispo; mais il n'y rivoit plus d'espoir: le Sultan venoit do f.) $i$ 
donner le duché à ce même Juif, nommé Jean Mịches, dont il avoit reçu de grands services, et quillut bien aise de récompenser.

Le duc prétendu n'osa pourtant jamais venir lui-même dans l'Archipel; il se contenta d'y envoyer un gentilhomme chrétien, Espagnol de naissance, nommé Françuis Coronello, qui gouverna sous son nom. Coronello étoit un homme de qualité, dont le père avoit été gouverneur de Ségovie sous le régne de Ferdinand et l'Isabelle : sa probité et sa droiture lui ayant attié de grand chagrins dans son pays, il résolut de le quiter, et de faire le voyage de Grice, dans le dessein de s'y élablir. C'est là quil fit conmoissance avec Miches. Jamais duc n’avoit encore éié plus chéri ni plus respecté que le fut Coronello durant tout le temps de son administration, qui ne finit qu'arec sa vie : il maria Coursin son fils à une des nièces de Jaeques Crispo. Sa famille s'est per pétuéc jusqu'à nos jours, et a produit des sujets d'un grand mérite.

Après la romination de Jean Miches, Crispoet ses enfans se refugrièrent à Venise. La république Jes recut avec de grandes marques de compassion et de iendresse; ct comme ils ćtoient dénués 
SUR L'ILE DE SYRA. CHA P. II. 25 de tout, on leur assigna des fonds suffisans pour les fuire subsister d'une manière conforme à leur naissance, et au rangy illusire qu'ils avoicnt tenu. Le malheureux dice neut pas la consolation d'en jouir long-temps; il mourut dans peu, accablé d'ennuis et de regrrets ; et cette famille, autrefois si considérable en (Jrient, est entiererement éteinte (1).

Ainsi finit la souveraneté de l'Archipel, l'an 1556, après avoir été plus de 300 ans entre les mains des princes latins. Le Juif Miches ne la garda que peu d’années; et depuis lui, elle a toujours relevé immédiatement du grand-seignemr. La Porte ne ticnt dans ces îles ni officiers ni gouverneurs, pour y présider en son nom ; éćtoit d'abord les premiers-desseins du divan, et chaque île considérable avoit son bey ou son cadi qui la gouvernoit, mais les armateurs chrétieusquicourentces mers, et qui s'y sont rendus terribles, leur faisoient tous les jours tant d'insultes, et en ont enlevé un si grand nombre, quils alloient vendre ensuite

(x) L'árchevêque actuel de Naxie esî un Crispo. Sa famille prétend descendre des dues de ce nom. 
on à Malthe ou à Livourne, qu'enfin les Turcs ont pris le parti de ne plus grouverner que de loin. Depuis ce temps-là claque île crée ses magistrats tous les ans, et fait comme une petite république à part. Ces magistrats se nomment Ejitropes : ils ont une autorité fort étendue; et à la mort près, quils ne peuvent ordonner , ils sont les maîtres d'infliger presque toutes les autres peines. Outre cela, ils ont soin de lever le tribut qu'on doit payer au grand-seigoneur. Dès que le capitan-pacha paroît arec sa flotte à Drio, port situé au sud-est de lî̀le de Paros, ils vont le tromver, et lui portent le tribut de leurs îles respectives.

Avant de finir le tableau de l'Archipel, nous dévons observer que les îles dépendantes ciu duclué de Naxie, en se soumettant à la Porte, ne négligèrent point de se réserver, par cette espèce de capitulation, dite en turc alid-name, (ahdveut dire traité ou serment, name, recueil), le libre exercice de la religion chrétienne; aussi jouit-elle dans ces îles d'une publicité de culıe, ¡jụj est généralement interdite dans les ćtats du yrand - seigneur, si l'on en excepte les deux prinripautés de Valachie et de Moldavie. Parmi les diversprivilegres assurés aux chrétiens insulaires 
pár ces capitulations, on doit remarquer la liberte d'avoir des clochers, de somer les cloches, de réparer les églises toules les fois qu'elles en ont besoin, et de les réćdifier şil est nécessaire.

Persome nignure les gुênes et les contradictions que les chrétiens, sujets de l'cmpire Ottoman, ceprouvent par-tout ailleurs sur l'article des répalations de leurs éğlises; la politique mahométane, quj en a éparghé quelquesunes, lurs de la conquête, tend insensiblement à les laisoer tomber en vétusté, et se rend extrêmement difficile à toutes les demandes qui paroissent contraricr ce principe.

Quant aux clochers, il in'en cxiste aucun dans toute l'étendue de la Tusquie; et pour inviter le peuple au service divin, les chrétiens n'ont que des crieurs publics, qui ront dars les quartiers habités par eux, annoncer les lieures de l'office; et dans les villages, ils se servent, pour le même effet, d'une planche sur laquelle ils frappent avec un marteau de bois.

Enfiu, il faut ajouter ici que tous les habitans des îles de l'Archipel, sont appelés par los 'iures Taouchan, qui véut dire lièvres. Cette dénomination, dit-on, a comnencé à Syra. Lors des ancieunes incursions des 'Tures sur la me! Palan= 
28 PRÉCIS HISTORIQUE che, ceux-ci firent une descente à Syra, les habitans n'étant pas en état de leur résister, gagnèrent le haut des montagnes. Les Turcs se mirent à leur poursuite, mais inutilement; alors ils les comparèrent à des lièvres, s'écriant: Taouchan guibi catchayeur, Ils fuyent comme des lievres; et le nom leur en est resté. 


\section{H A P I T R E I I.}

De l'état ancien de l'île de syra.

Sy R A ou Syros (1), comme l'appeloient les anciens, est une île de la mer Égrée ou Archipel, située presque au centre des Cyclades (2),

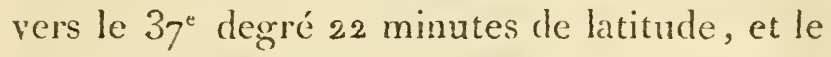
$42^{\circ}$ degré 14 minutes de longitude. Sa longueur du N.O. au S. E. est d'environ 14 milles, et sa largeur de l'O. à l'E. de 6 à 7 milles; ce qui lui donne au moins 4 o milles de côtes, quoiqu'on ne les estime communément qu'à '36 dans

(1) Homère lui donne encore le nom de sugic; mais j'observerai qu'on ne doit jamais écrire Sciros, ni confondre cette île avec une autre qui est au N.E. de l'ile de Négrepont, et qu'on doit appeler Skiros, comme font les Grecs modernes. J'observerai encore avec Tournefort, que le nom de Syros ayant été de temps immémorial celui de l'ille, c'est se tromper lourdement que de dériver son nom actuel du grec vulgaire, sugús ou xugía, dame, maitresse.

(2) Elle est entourée des îles de Naxie, Paros, Siphanto, Serfo, Zea, Jura, Andros, Tine, Mycone et Délos. 
le pars. Malgré son peu d'étendue, elle n'al pas Iaissé d'avoir quelque célébrité dans l'antiquité la plus reculée. Elle a cu des rois particuliers, comme presque tontes les autres iles de l'Archipe!. Ctesius, fils d'Ormenus et pìre d'Eumće, étoit Roi de Syra, comme on le voit dans 10dyssée, XV. Elle étoit déja renommée dị temps d'Homere, par sa fertilité en blé et en vin, par le grand nombre de ses bestiaux, par la pureté de l'air qu'on y respiroit : et par un monument astronomique qui y indiquoit les solstices et les équinoxes. Voici les paroles que ce prète met dans la bouche d'Lumée, s'entrenaut avec U lysse dans l'ile d'Ithaque: "An-delà de l'île d'Ortygie " est une île appelée Syrie; si jamais vous avez * entendu ce nom, c'est dans cette île que se " voient les conversions di soleil : elle n’est pas "fortyrande, mais elle est fort bonne; on y nourrit " de nombreux troupeaux de brufs et de mou" tons; elle rapporte beaucoup de vin, et une " grande quantité de froment : jamais la famine " n’a désolé ses peuples, jamais les maladies " contagieuses n'y ont fait sentir leur venin. Ses * habitans ne meurent que quand ils sont par" venus à une extrême, vicillesse et alor's, c'est "Apollon lui-même, on Diane sa scem, qui ter- 
" mirent leurs jours avec leurs doucesfleches. "Il y a dans cette île deux villes qui partagent "son territoire. Mon père Ctesius, fils d’Ormé"nus, scmblable aux immortels, en étoit roi." Ce que dit Homère de la fertilité de Syra, et de la salubrité de l'air qu'on y respire, exempt de toutc influence maligne, prouve que ce poète étoit parfaitement instruit de la nature de cette île, et de l'origine du nom qu'elle porte encore; car, comme Bochart le fait voir, c'étoient les Pléniciens quil'avoient ainsi nommée, du mot Syra, pour usira, qui signifie riche; on plutôt du mot Sura pour usurz, qui vent dire heurense : ces deux mots marguent également la bonté de son terroir. Je dis qu'Homère a connu la véritable origine du mot syros : la preuve la plus incontestable qu'on en puisse apporter, c'est ce qu'il ajoute du séjour des Phéniciens dans l'île. Quant aux conversions du soleil dont parle le poète, nous expliquerons dans le chapitre suivant ce qu'ib fáut entendre par-là.

L'île produit encore aujouri'hui assez de grain, de vin, et d'autres denrées; mais il s'en faut de beaucoup qu'clle ait conservé la fertilité des premiers temps. Outre que les principes de 
végétation vont toujours en diminuant d'activité par la succession des siècles, il y a lieu de. croire que les montagnes ct les collines qui occupent plus de la mo tié de l'ile, étoient autrefois couvertes de terre végétale, et qu'en augmentant sescultures, elles augmentoient aussises productions et ses richesses. Mais cette terre aura été entraînée, peu-à-peu ou subitement, par l'eau des pluies, et les montagnes restées nues seront devenues stériles. Quelques vallons mêmes des plus fertileş et des plus unis, ont éprouvé cette révolution, et les pluies tombées au commencement du siècle les ont tellcment sillonnés, que les torrens y roulent aujourd'hui comme dans leur lit.

Au surplus la salubrité de l'air y est la même que du temps d'Homere. Les vieillards de 80 , 90 jusqu'à 100 ans y sont assez communs; il n’y a pas long-temps qu'il y en avoit un de 115 ; et un fait qui passe pour constant dans le pays, c'est qu'une personne quíy a passé 45 ans, est pres. que assurće de parrenir à 70,80 et au-delà : enfin les Syriotes ont une ancienne tradition qui vient à l'appui de cette opinion, et de ce qu'Homère raconte de la longne drrée de leur vie et de leurgenre de mort; c'est qu'autrefois, quand 
les vieillards étoient arrivés à la dérrépitude, leurs parens les menoient sur une haute moutagne, d'où ils les précipitoient pour les faire enfin mourir. Cette montagne s'appelle encore Jerusi, montagne des vieillards.

Le partage du territoire de l'ille en deux villes, dontparle le poète, est pent-être représenté par ce qu'on appelle aujourd'hui la partie du dedans

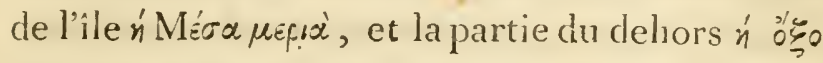
$\mu \varepsilon f \ltimes \alpha^{\prime}$, et parmm mur qui, selon la tradition, prenoit d'une merà l'au tré,et divisoit l'île en deux portions. Outre ces deux villes, il y avoit plusieurs villages, comme l'indiquent les ruines que l'or voit en plusieurs endroits différens: les plus remarquables sont auprèsdu port; on y distingue une assez grande villesur me éminence qui étoit au bord de la mer dans la plus belle position, mais trop exposée aux incursions des pirates; c'est pour cela que ses habitans l'ont abardonnée, et ont été bâtir la nouvelle ville sur une montagne voisine. Une inscription tirée déces ruines, et encastrée dans un coin de l'église de Saint,George, la cathédrale actuelle, nous apprend que l'ancienne ville s'appeloit Syros. A gauche de la partie de l'évêché, sur un bas-relief de marbre, est représenté le sistre des anciens, avec quelques autres Tome $I$. 
instrumens. C'est eucore un monument tiré des mêmes ruines. On en a mis à profit beaucoup d'autres, telles que des blocs de marbre, des tronçons de colonne qui soutiennent la cathédrale, et d'autres qui sont devant l'église des capucins, et qu'on n’a point encore employés. II reste cricore sur le lieu un pan de muraille, bâti avec de gros quartiers de marbre bâtard taillé à facettes, qu'on croit avoir servi à un temple dédié an dicu Pan, d’après des médailles sur lesquelles on voit la tête de ce Dieu d'um côté, avec une chève au revers. Enfin il n'y a pas long-tcmps qu'en labourant près de là, on a trouvé un beau buste antique, que l'on croyoit. être du philosophe Plaérécyde : ceétoit pendant que la flotte russe croisoit dans l'Archipel. M. Janulachi Salacha, alors épitropos de Syra, qui étoit possesseur de ce buste, en fit présent au commandant Russe. Il faut conjecturer d'aprèsce que nous venons de clire, que si on se donnoit la peine de fouiller, on pourroit encore tiouver de belles antiquités; mais pour le faire avec sureté, et n'être point exposés aux insultes des Turcs, qui croient toujours qu'on cherche des trésurs, il fauclroit travailler sous le nom d'un 
SUR L'íle DE SYRA. CHAP. III. 35 consul-françois ou des PP. capucins, qui jouis. scnt des mêmes liranchiscs.

Nous terminerons cet article par une tradition qui se conserve parmi ses habitans. Quand les gros temps ou les vents contraires empêchoient les pélérins d'entrer dans le port de Délos, et les obligeoient de relâcher dans celui de Syra, qui est vis-à-vis, sil voyoient que ces mauvais temps durassent trop, ou que leurs at: faires ne leur permissent pas d'attendre, après s'être purifiés dans l'eau de fontaine, que l'on entretenoit pour cela tout près du port, ils se reudoient au sommet d'une petite montagne, voisine quiregardoit Délos, et de là ils adressoient à Apollon leurs adorations, leurs offirandes et leurs sacrifices. Cette montagne s'appeloit pour cette raison montagne de Délos, et nous l'appelons encore $\Delta \dot{n} \lambda$ s. 


\section{H A P I T R E. I I I.}

De l'ancien monument astronomique de Syra, et du philosophe Phérecyde.

L E monument qui indiquoit les solstices et les équinoxes, et la naissance du philosophe Pliérécyde, l'un des plus fameux philosophes de son temps, étant les deux points de l'histoire de Syra qui la distinguent le plus, il me semble quils doivent trouver maturellement leur place ici, d'autant plus qu'ils sont en général peu connus. Par rapport au monument astronomique, nous examinerons la note de madame Dacier sur ce not d'Homère: c'est-là que se voient les consersions du Soleil. "Voici, dit cette savante Dame, " un passagctrès-important. M. Despréaux, dians "ses réflexions sur Longin, a fort bien réfuté * la ridicule critique que l'auteur du parallèle, * homme très-ignoraut en grec et en latin, et. " sur-tout en géographie, avoit faite contre Ho" mère, e'cst-à-dire contre le père de lá géo"graphie, en l'accusant d'ètre tombé dans la "plus énorme bévue qu'un poète ait jamais 
SUR L'I LE DE SYRA. CIA P. III. 37 " faite; c'est, dit-il, d'avoir mis Syros et la mer "Méditerranée sous le tropique; bévue, ajoute* t-il, que les interprètes d'Homère ont tâché en * vain de sauver, en expliquant ce passage par " le cadran que le philosophe Phérécyde, qui " vivoit 300 ans après Homère avoit fait dans " cette île. Mais je suis fàché que M. Des" péraux, qui réfute cette misérable critique " avec tant de raison et de fidélité, ne soit pas " entré lui-même dans le véritable scns de ce " passage, et qu'il se soit laissé trompor par une " note d'Eustathe, qui lui a persuadé que ces

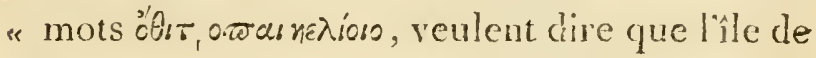
* Syros est au conchant de Délos; car c'est * ainsi qu'Eustathe l'a d'abord expliqué xusém

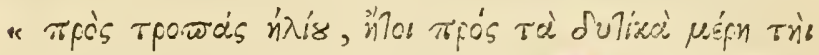
* op7rgias, ete. c’est-à-dire quie Syros, est situéc " au couchant du soleil, au couchant de lîle "d'Ortygie; car tpéroesia, le loumar, se dit du "soleil pour duveiv, se couclicr. M. Despréaux * devoit voir que cette explication étoit in" soutenable, car il est absolument faux que "l'île de Syros soit au couchant de l'île de Dé* los : aucun ǵograplie ne l'a jamais dit; * et comment Homere auroit.il pu le dire dans « le même vers où il a dit Op puzins ratjúropins, 
" au-dessus de l'île d'Ortygie? ce qui est au delà " ou au dessus de cette île, pour Eumée qui est " à Ithaque, ne peut jamais être au cory"chant."

Voici comment parle le savant Bochart, dans sa géographie intitulée Chanaan, lib. I , cap) 1 4: «Eustathe se trompe quand il veut que " parndis tporás on entende le couchant, comme " si l'ile de Syros, étoit au couchant de Délos; " car au contraire elle est au levant, et non au " couchant de cette île. C'est la situation que lui " donnent les géographes, et il ne faut que ce "vers d'Homere pour prouver que c'est la vé"ritable posilion, puisque Eumée qui est à Itha"que, assure que Syros est au dessus, au delà " d'Ortygie; ce qui seroit très-faux, si elle étoit "au couchanit de Délos. Eumée auroit plutût - " clû dire en deçà. Il fálloit cionc s'en tenir à la * seconde :xplication qu'Eustathe a ajoutée dans "la même remarque. D'autres, dit-il, expli"quent ce passage, en disant que dans l'île de "Syros il y avoit un antre qui marquoit les "conversions du soleil, c'est-à-dire les sulstices, " et quion appeloit l'antre du solcil pour cette " raison. Et voila ce quentend Homere par ces "mots: Oí sont les conversions du soleil ; i Voilà 
SUR L'île de Syra. Chap. III. 39 "la seule véritable explication; elle mérite "d'être éclaircie."

"Nous voyons, par ce passage même, que les "Phéniciens avoient fait un long̈ séjour dans "lîle de Syros; nous verrons plus bas que les Phé"niciens lui ont eux-mêmes doané son nom, et "nous savons qu’ils étoient très-savans en astro"nomie : c'est de là qu'il faut tirer l'explication

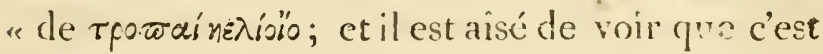

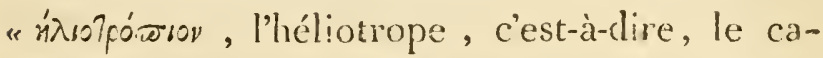
" dran; et par là Homère nous apprend que les "Phéniciens avoient fait clans cette île un ca"dran, dont le style on aiguille, par le moyen " de son onbre, indiquoit les solstices; et comme "cétoit une chose fort rare et merveilleuse dans * ce tomp--là, Homère, fort curienx et bien ins" tuvit de tous ces points d'antiquité, la marque " comme une rareté qui distinguoit cette île. "Bicntôt après, les cadrans devinrent plus com"muns. Environ six-ringts ans apres Homère, "l'écriture-sainte fait mention, res. iv, cap. 20, * v. 11 , d'un cadran qui étoit à Júrusalem, et "qu'on appcloit le cadran d'Achaz, sur lequel "Dien fit, en faveur d'Ezéchias, que l'ombre " rétrograda de dix degrés. Ce cadran mrquoit "les heures et les solstices." (Nous allons prou- 
ver le contraire). " II y avoit donc des cadrans " avant celui de Phérécyde, qui ne fit le sien « à Syros que 200 ans après celui d'Achaz, et 300 * ans aprés celui des Phéniciens ; et par consé"quent, pour expliquer ce passage d'Homere, " on n'a eu recours qu“à ce cadran des Phéni" ciens, et nullement à celui de Phérécyde, que "le poète n'a pu connoître. Il me semble que " cela est prouvé; mais il y a plus encore, c’est "qu'il y a bien de l'apparence que ce cadran "que Phérécyde fit à Syros, ne fut fait que sur "les cléconvertes des Phéniciens; car Hésychius " de Milet, dans le live qu'il a fait de ceux qui " ont été célèbres par leur ćrudition, nous assure "que Phérécyde, quiétoit de Syros même, n'ent "point de maitre, et qu"il se rendit habile en "étudiant quelques livres secrets des Phéni" ciens qu'il aura recouvrés. Je me fiatte, conchut "Madame Dacier, que ce passage d'Homere " est assez bien éclairé. "

Quelque respect que íaic pour l'cirudition de cette savante Dame, l'amour de la rérité ne me permetra cependant pas d'être de son avis, parce que, tant elle que Buchart, ct tous les géographes qui placent Syra à l'orient de Délos, se trompent. Ptolomée, dans sa carte de la Gréce 
SUR L'Ile de Syra. C'hap. III. 4I et de l'Archipcl, tombe dans la même erreur. Mais pour s'assurer de la vérité, M de. D’acier et Bochart n'avoient qu’à écrire en Provence, où le premier marin qui avoit visité l'Archipel lui auroit appris que Syros est vers le couchant de Délos. Pour moi, je puis assurer que ma maison à Syra regardoit Délos, de manière que quand le matin, au mois d'uctobre et au mois de février, je me mettois à ma fenêtre ou à ma porte, le soleilen se levant derrière cette île, me donnoit précisément en face. Le soleil, par rapport à Syra, monte l'été jusqu'an petit canal qui sépare lile de Mycone de celle de 'Tine et l'hiver, il descend jusqu'aux montaynes de Naxie. Or, les deux Délos, la griande ct la petite, se trourent, parrapporta Syra, entre Mycone, et Naxie, et plus même du côté de My cone; de sorte qu'une partie de cette dernière est cachée par la graude Délos. Aussi M. de Toumefort, dans son royage du Lerant, et tous les géographes modernes d'après lui, placent Mrcone à l'E. de Syra; la zrande Délos entre l'L. ct le S. E. : d’oul l'on voit qu'Eustathe ne se trompoit pas, lorsqu'il disoit qu'on pourroit entendre les paroles du poète, comme sil disoit que Syra est au couchant de Délos, et cue son opinion niest pas insontenable, 
comme elle le seroit effectivement si Syra étoit à l'orient de Délos. Cependant cela n'empêche nullement d'expliquer le texte d'Homire, comme on l'explique communénent, et comme Eustathe le fait en second lien, par un monument quelconque qui existoit à Syra, et qui rendoit cotte île célćbre depuis quelque temps; ct la difficulté qu'apporte $\mathrm{M}^{\mathrm{de}}$. Dacier de cette expression, 'Optuzins xxqúmerãè, au dessus de l'ile d'Ortyurie, ne s'oppose en aucune manière à cetto explication. Voici commient. Naturelicment parlant, la partie du globe la plusćlevée, c'est l'équateur, et les deux pôles forment ses parties latérales; q’où il résulte que tont pays plus voisin de l'équatcurqu'un autre, est aussi plusćlevé, et par conséquent au dessus. Or. Syra, quoique placée an couchant de Délos, est en mẻine temps plus méridionale ou plus rapprochée de l'équateur que cette île et Ithaque, que nons appelons aujourd'hui Thiaki. Parconséyuent, tant par rapport à Ortygie or Délos, que par rapport à Ithaque, Homère a pu dire que Syra est au dessus d'Ortygie. de n'oserois pas dire qu'Homìre, dans la chaleur de la composition, préoccupé et faisant plus d'attention à l'endroit d'où il écrivoit (lîle de Chio, selon la plus commune 
SUR L'íle de Syra. Chap. III. 43 npinion ) qừ celui où il plaçoit son interlocuteur, a écrit que Syra étoit au-delà d'Ortyguie. Mais passons à Phórécyde, dont l'histoire extraite des Mémoires de l'académie de Berlin, nous instruira mienx de la nature de ce monument astrunomique. Phérécyde de Syra commenca à fleurir dans un temps où la philosophie étoit cncore dans son enfance; car les amnates portent que ce fut la réputation de Thalés de Milet en Ionic, fondateur de la secte ionique, qui lui inspira le desir de se distingner dans la même camèere. Or 'Thales étoit déja célèbre dans la $19^{\circ}$ olympiate, puisqu'bl aroit prédit une écipse de soleil; et l'auteur des Otrmpiales remarque que Phéiécycle naquit dans la $+j^{\circ}$ olympiade, dont la prenriére année répond à l'an 5,8

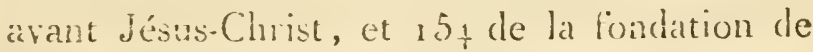
Rome. Nons pouvons donc placer sans difículté l'ige de notre philosophic lans le $6^{e}$ siecle avant Jesus-Christ, et Pline, hist. nat. 7.56, a en raison de dire que Phérécyde de Syra florissoit du tomps de Cyrus.

Phérécyle prit le surnom de Syrien de l'ile oì il étoit né; Grotius le fait venir de Syrie. saus en alléruer aucune preuve. Cependant il y a beancoup plus de raison de donner, avec la 
grande partie des anciens, pour patrie à ce philosophe, l'île de Syros dont nous parlons. C'est ce que dit expressément Strabon. "Syros (l'île) "a la premièresyllabe longue. C’est dé là qu'étoit "originaire Phérécyde, fils de Babis, qui est plus "ancien que Phérécyde l'Athénicn; or, Syros , "ajoute-t-il, est l'une des Cyclades. Homère vante "beaucoup sa fertilité et son air salubre. "

Quantau nom de cette île, Homère dit Eupıa; Suidas, Syra; Strabon et les autres, Syros. On peut donc appeler Phérécyde indifféremment $S y-$ rius ou Syrus. On écrit actuellement Syra, le nom de l'île, et Syriotes celui des habitans; et dans le style de Rome, l'évêque de Syra est appelé cpiscopus Syrensis, pour le distinguer d'un évêque de la province de Syrie, que l’on désigne par episcopus syrus.

Les écrivains ne s'accordent guère sur l'éducation ct sur les maîtres de Phérécyde. Diogène Lac̈rce tombe dans une erreur qui n'est presque pas pardonnable, en faisant Phérécyde disciple de Pittacus, dont la naissance répond à l'an de Rome ror. Il parvint au souverain commandement dans,sa république à l'âge de 46 ans. p'cut-onimagyiner qu'un prince, qu'un législateur, se soit occupé, ¿̀ 70 ans au moins, à donner des 
SUR'L'ILE DE SyRA. ChAP. IIT. 45 icçons à la jeunesse : car Phérécyde ne vint au monde que l'an de liome i 56, c'est-di-dire, dix ans après que Pittacus eut commencé à régner sur les Lesbiens. Il faut donc rejeter ce que Vossius avance aussi, qu’il fut le maître de Phérécyde. Tzetze a une autre opinion; il fait Thales disciple de notre philosophe. Si nous croyons Apollodore, cité par Diogène, Thalès naquit l'an de Rome I 4 ; ainsi il avoit déja 40 ans lorsque Phérécyde vint au mondc. J’admettrai donc bien plus volontiers ce que dit Sirdas, que la gloire de Thalés excita l'émalation de Phérécyde. Hésychius, dans un petit ouvrage qu'il a écrit sur Jes personnages illustres par leur savoir, met Phérécycle au nombre de cenx que les Grecs appeloient Au7odidax7\&s, c'est-à-dire, qui se sont instru's eux-mêmes. "On assure, dit-il, " que Phérécyde de Syrie n’ent ancun maître, " mais quil se poussa lui-même dans les scien«ces, après avoir trouvé quelques onvrages ca" chés, ou commentaires secrets des Phéniciens. « Suidas et Eustathe dirent la même chose.

Le principal point de controverse des critiques regarde ces livres des Phéniciens. Homère a dit formellement que les vaisseaux Phéniciens abordoient à lile de Syros, et Bochart 
46 PRECIS HISTORIRUE prend de là occasion de prouver plus au long que les Phéuiciens fréquentoient l’île de Syros dès les temps héroïques, et y passoient souvent une année entière pour leur commerce. Il n'y a done pas lieu de s'étonner qu'un homme avide d'apprendre, en s'entretenant fréquemment avec les étrangers et les négocians, ait oui parler de livres pleins d'une connoissance profonde et sublime, et se seroit enflammé du desir de les posséder. Mais la question est tou. jours de savoir quels sont ces livres, qui tombèrent entre les mains de Plérécyde.

Lillustre évêque d'Avranches , M. Huet (Demonstr. évang. proj. w, S. 7, ) nous a heureusement tirés de tout embarras. "Phéré"cyde, dit-il, fut disciple des Esyptiens et "des Chaldéens, mais sur-tout des Phéniciens, "des livres secrets desquels ont dit qu'il tira * une grande conmoissance des choses divines, " n'ayant point eu d'ailleurs đ'antres maîtıes. * J'entends par ces livres secrets des Phéai"ciens, ceux de Moyse, auxquels Juvénal "donne !e nom d'Arcanum. On les attribue aux "Phéniciens, qui, comme je l’ai dẹja remar"qué plus d'une fois, sont souvent pris dans "les auteurs profanes pour les Juil's leurs 
SUR L'île DE SYRA. Chap. III.

"voisins; ou bien on peut entendre par là l'é" crit de Sanchoniaton, qui aroit élé tiré des li"vres de Moyse. "Il est done très-probable que Phérécyde s'est instruit avec les livres de Moyse, ou de quelques autres faits sur les livies de Moyse.

Ceux de nos lecteurs qui seront curjenx de comnoître la vie, les écrits eţ lesopinions de notre: philosophe, pourront consulter les Mémoires de l'académie de Berlin, anuée i 7 77. Nous ne donnerous place ici qu’à ce que ces Mémoires nous apprennent sur flhéliorrope, qu'il a élevé dans sa patrie, et sur sa mort.

Phérécyde done, suivant le témoignage de Diogène Laërce, enrichit sa patrie de l'héliotrope, comme Anaximandre fit à Lacédémone le premier gnomon qu'on ait vu en Grèce, et comme Méton, après Anaximandre, en contruisit un dans le חi: l'une des places publiques d'Athènes. Or l'béliotrope est une espéce de cá dransciatérique (de oxsò, ombre), un instrument dont on se sert pour mesurer l'ombre, sur-tout aux solstices, et déterminer par sa longueur les solstices mêmes, qui sont ce que nous entenclons

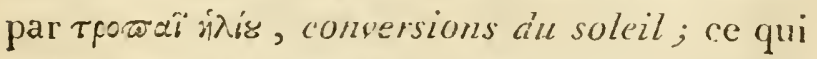
étoit d'autant plus aisé, que, comme tout le 
monde le sait, tous les peuples placés comme nous entre la ligne équinoxiale ct le pole arctique, ont leur ombre la plus courte au solstice d'été, et la plus longue au.solstice dhiver. Mais Pline parle d'un autre moyen dont on se servoit encore pour connoitre les solstices. On " rapporte dit-il, qu'à Syene qui est à 5ooo stades "au-dessus d'Alexandrie, le jour du solstice, " à midi, il ny a point d'ombre; et qu'un puits "qu'on a creusé pour cette expérience, se troure "éclairé dans toute sa profondeur; ce qui lait "voir que le soleil est vertical dans ce lieu. " Il y avoit done à Syene une espèce d'béliotrope naturel, qui promsit que cette ville étoit placée précisément sous le tropique du Cancer. Tel étoit à peu-prís celui de Syros, dont Homère fait mention, sur quoi le Scholiaste remarque fort bien. "On dit quil y a une caverne du soleil "par le moyen de laquelle on remarque les con"versions de cet astre."

Le père Hardonin débite des choses tout-à"fait déraisonnables, en disant, que « le porcher "d'Ulysse qui étoit à Ithaque, ne vouloit expri* mer autre chose par ces paroles, si non que "l'île de Syros étoit au-delà d'Ortygie, et que "quand le soleil se lève à Syra, il se montre, 
SUR L'île de Syra. Chap. III.

oic est sur le point de se montrer aux habilans d'Ithaque. Syrosestà lorient du Péloponèse, ec Ithaqne est à l'occident. On ne sauront attribuer des idées plus profondes à un porcher. Il est srai qu'Euméc éloit porcher clars le temps qu'il tint ce discours à Ulysse; mais c'étois le fuls d'un roi de l'ille de Syros, que les Phéniciens avoient autrefois enlevé et vendu i Laërta père d'Ulysse. Le fils du roi pouvoit-il ignorer les particularités de sa patrie? Ainsi, répond $\mathrm{M}$. Heinius, auteur de la dissertation d'où j’ai tiré la plus grande partie de ce que je viens de dire sur Phérécyde, je crois, au contraire, que l'on pourroit ajouter, que quoique Homère fasse parler ici Eumée, il pent très-bien faire qu'il ait oublié un moment son interlocuteur, pour raconter les faits comme il les savoit lui-même, plutôt que comme Eumée devoit les savoir.

"Si Homère, continue M. Heinius, avoit "vóulu simplement indiquer par tpordais inisio, " le leverdu soleil, qu'auroit-il cu besoin d'ajou* ter Syros à Délos ? Celle-ci inême n'éloit-clle "pas à l'orient de l'autre?" "M. Heinius, comme on voit, savoit à Berlin, c'est-à-dire, dans un pays qui a peu de moyens de communication Tome $I$. 
avec l'Archipel, ce que $\mathrm{M}^{\mathrm{de}}$. Dacier a ignoré en France, que Syros est ì l'uccident de Délos. En suivant le principe du P. Hardouin, il y auroit cu des7powá míso dans tontc's lesîlestle la mer Egée, ce qui seroit tout-à-fàit contraire à l'intention d'Homere. Donnons done plutôt des éloges au Scholiaste, qui nous a indliqué dans notre île une caverne naturclie, et non crensée par l'art, qui servoit d'héliotrope.

L'astronomie ayant pris dans la suite des accroissemens, on inventa le gnomon ou cadran, par le moyen duquel on mesure et on partage l'ombre. La forme la plus ancienne du gnomon étoit celle d'une pyramide quadrangulaire équiJatérale, terminée par une boule, dont on trou*era une plus ample explication dans les notes de Saumaise sur Solin. Je ne doute pas non plusque les pyramides d'Egypte ne fussent destinées, entre autresusages, à marquer la gradation de l'ombre : car ce que quelques anciens ont prétendu, en soutenant que les pyramides n'en jetoient aucune, est pleinement réluté par les"savans auteurs'de l'histoire universelle. On explique aisément par là pourquoi la plus grande des pyramides a ses quatre côtés exactement tournés vers les quatre points cardinaux de la 
Sur l'île de Syra. Chap. IV. 5e sphère, et pourquoi elle s'éleve par deçrés; cétoit pour observer aves plus d'exactitude les degrés d'accroissensent et de décroissement de l'ombre. Ce que nous venons de dire pourroit pent-être jeter que!que jour sur un passage extrêmement débattu, celui du cadrar. solaire, ou plutôt des despés d'Achaz, et de l'ombre qui retoumuit par ces degrés. Reg. riv cap. 20 , rar. so et I I. Les plus célèbres critiques ont donné à l'envi leurs explications sur ce passage. V'oici mon opinion. Je crois qu'on ne doit pas entendre par ces degrés une horloge solaire qui divise les jours en vingt-quatre heures équinoxiales, c'est-à-dire, égales pour le jour et pour la nuit; cette division étoit inconnue du temps d'Achaz, et l'invention de pareilles horloges est de beaucoup postérieure à ce Roi. D’ailleurs les cadrans solaires ne sont pas faits pour distingrer l'alongement et le raccourcissement de l'ombre, mais seulement la manière dont clle tourne autour du gnomon. Or il n'étoit question dane les degrés d'Achaz, que de la mesure de l'ombre; et c'est précisément à quoi pourroit servir l'héliotrope, dans lequel l'ombre croît journcllement depuis le solstice d'éié jusqu'd celui d'h:ver, et décroît dans la même pioportion depuis 
le solstice d'hiver, jusqu'à celui d'été. Tous les joursavant midi l'ombre montoit et se raccourcissoit, et l'après-midi elle descendoit et s'alongeoit. Cela nous explique en corcles paroles d'Ezéchias. Il cst plus aiséà l'umbre de baisser de dix degrès, que de remonter d'aulant de degrés. Mais pourquoi cette facilité d'un côté, et cette difficulté de l'autre? aucun interprète que je sache n'en donne la raison. Supposons donc que ce soit l'après-midi que le prophète ait été trouver Ezéchias : le solei! , qui suivant sa marche naturelle descendoit alors vers le couchant, alongeoit à chaque instant l'ombre dans les clegrés. Ezéchias aimoit donc mieux avoir pour garant de la promesse qu'luaie lai faisoit, un miracle, c'est-à-dire, l'ascension de l'ombre, qui ne poufoit se faire à cette heure suivant l'ordre de la nature. Mais finissons cette digression, pour revenir à Phérécyde, et concluons que trouvant dans son île un schiothérique, il le perfectionna en y adaptant un gnomon; et que c'est avec raison que Diogène Laërce lui en a fait honneur. Passons à la mort de ce philosophe, dont nous arons promis de dire quelque chose.

Ce r'est pas une médiocre gloire pour lui que. d'avoir eu un disciple tel que Pythagore, que la 
SUR L'île DE Syra. Cha P. IV. 53 philosophic a reconnu pour un de ses plus grands maîtres. Toute l'antiquité est d'accord sur ce fait, qu'elle nous a transmis non-seulement par tradition, mais même par un monument consacré à l'immortalité. M. Béger, habile antiquaire, dans son Spicileginun antiquitatis, pag. 136, cite deux tableaux tirés de Pighius, qui représentent l'enfance et la première adolescence de Pythagore: on voit dans le second ce philosophe, tout jeme encore, et avide d'ap-

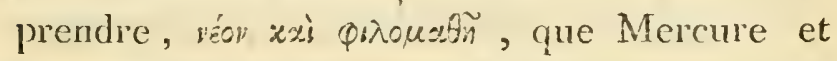
deux muses présentent à Phérécyde, pour en recevoir les premiers élémens des sciences. Dans la suite, Pythagore a tellement surpassé son maître par ses connoissanees et par sa célébrité, qu'il a cntièrement éclipsé sa renommée et scs érrits, et peu s'en faut que Phérécyile ne soit entièrement oublié.

Mais un point sur lequel les auteurs sont partagés, c’est le lieu où mourut Phérécyde, et la manière dont il mourut. Quelques-uns, au rapport de Diogène Laërce, croient qu'étant allé à Dolphes, il se précipita du haut du mont Corycins; mais Aristoxène, qui parle de Pythagore et de ses amis, rapporte que Phérécyde mourut de maladie, et fut enseveli par Pythagore dans lile de 
Délos. Or, Aristoxène est qualifié dans AuluGelle d'écrivain très-exact sur les faits de l'antiquité, vir litteramum diligentissinns ; et nous allons voir ce récit confirmé et développé dans Elien, var. hist. lib. 1v. 28. "Phérécyde "de Syros, dit cet historien, mourui de la ma"nière la plus misérable du monde. Tont son " corps fut rongé par les pous, el son visage " tellement déflgmé, qu'il n'osoit plus se mon* trer à ses amis, et qu'il les évitoit avec soin. "Cependant un d'entx s'étant un jour présenté "à sa porte, el lui ayanidemandé comment il " se portoit, Phérécyde lnifit voir par un tron, "sorz doigt lout décharizé, et lui dit que tout "le resı de son corps étoit de même. "Elien décrit un peu plusbas (lib. v., 2.) toute l'horreur de ce mal en ces termes: "Pliéréryde, mầ" tre de Pylhagore, étant lombé malade, Irans"plioit d'abordune srrentr chande, et semblable * à des mucosités, qui le jeta ensuite dans la "pluhiriase. Tontes ses chairs se réduisirent en "pons; la cornuption s'y mit, el it.finit ainsi " ses jonrs. " Mais Pythagore, bon juge des vertus et des devoirs, crut qu'il étoit d'un disciple reconnoissant de ne pas abandonner un maître aftligé d'une si triste maladie, et qu'il devoit au 


\section{SUR L'île dE SyRA. CHAT.IV. 55}

contraire en prendre soin jusqu'au dernier soupir, et même au-delà du trépas. Diodore de Sicile nous apprend comment il s'acquitta de ce pieux devoir. "Ayant appris, dit-il, que Phé"récyde étoil dangerensement malade dans l'ỉe " de Délos, Py.lhagore quiua anssitút l'Tralie, "pour se rendre anprès de lui, et le serwit guci. "que temps, en frisanl toul ce que l'art el sa "tendresse lui indiquoient ponr hii procurer la "santé. Mais "âge a la force du mal ayant " rendu lons ses soins inuliles, il lui donna " une sépulnure lionnîle, et après avoir ainsi " tenu en lont la conduile d'un fils à l'égard de "son père", il repassa en Ilalie. Divel. Sic. in escerpt.

Jacques Gronovius, qui a dirigé le Trézor des antiquités grecques, donne, d'après les tableaux de Béger dont nous arons parlé, un portrait de Phérécyde, qu'il in fait parfaitement graver. Toyez les antiquitís greeques, tome 2, fig. 37 ; Il y a joint un éloge de ce philosophe, par lequel nous terminerons ce paragraphe. "La Provi"dence divine, dit-il, aynnt fait conconrir * plusieurs choses à la gloire de ces remps-là, " (il parle du siècle de Phérécyde) aucune * particularité ne doil êlre négìisée, quelque lé- 
56 PRÉCIS HISTORIQUE "gère qu'elle nous paroisse, et de quelque " manière qu'clle nous soilparenue. Puis donc "qu'il nous reste un monument, non pas à la "vérilé de la plus haule antiquité, qui nous "représente avec nue physionomic belle et remar"quable ce philosophe de l'ile de Syros, si "illustre par ses actions el par ses écrits, et "qui lient le premier rang parmi les sages de " son lemps, j'ai cru devoir placer ici ce mor" ceau."

En effet, le buste qui a été trouvé il y a quelques années à Syra, parmi les ruines de l'ancienne ville, représentoit un philosophe d'une très-belle figure, ayant l'air majestneux; et ce passage de Gronovius me confirme encore dans la persuation où nous étions alors que ce monmment représentoi Phérécyde. 
SUR L'île de SyRa. Chap. IV. 57

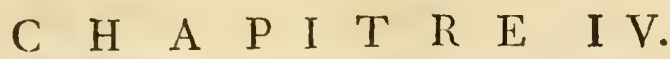

Etat actuel de lile de Syra.

Tournefort, dans ses Vorages du Levant, sexprime ainsi sur l'état actuel de cette île: «Syra est l'île la plus catholique de tout l'Archipel. Pour sept à huit families du rite grec, on y compte plus de six milie ames du rite latin; et lorsque les Latins sallient avec les Grecs, tous les enfans sont catholiques romains; au lieu qu’à Naxie, les ģarçons suivent le rite du p’ere, et les filles celui de la mère. On est redevable de tous ces biens aux peres capucins françois, missionnaires apostoliyues, fort aimés dans cette île, et fort appliqués à instruire un peuplc porté au bien, honnête, enuemi déclaré des voleurs, plein de bonssentimens, ct si laborieux, qu'on ne sauroit reposer dans cette île la nuit, à cause du bruit universel des moulins à braș que chacun exerce pour moudre son blé; et le jour, ì ciuse des rowets servant a filer le coton.»

"La maison et l'ónlise des capucins sont asecz bien bâties: la banière de France arboréc au coin de lemr terrasse nous séjouit; et le ficre 
Jacinthe d'Amiens, homme d'esprit, substitut du consul de France à Tine, nous reçut avec tous les agrémens possibles. Ces pères dirigent vingt-cinq relighieuses du tiers-ordre de Saint François, filles d'une vertu exemplaire, quoique non-cloitrées. Les Grecs n’ont que deux églises dans Srra, desservies par un papas. Il n'y a de 'Turcs qu'un cadi, encore vient-il se réfugier chez les capucins, lorsqu'il paroît quelque corsaire autour de lîle. On y élit tous les ans deux arlministrateurs. En I700, la capitation et la taille réclle montoient à 4000 écus. »

* Syra n’est qu'environ à 30 milles de Mycone, si l'on compte d'un cap à l'autre; mais il y en a 40 du port de Mycone à celui de Syra. Son port cst bon pour les plus gros vaisseaux, et son entrée est à l'est. Lîle, qui n’a que 25 milles de tour (Syros quan circiler patere viginti millia passum prodidere veleres, Mulianus cenllm sexaginla), est des mienx cultivées, et produit d'excellent froment, quoiqu'cu petite quantité, beaucoup (lorge, beancoup) de vin et de figues, assez de coton, et des olives que les habitans salent pour leur usage. Qnoique Syra soit un île montagueuse, clle manque de bois, et l'on n'y brûle que des broussailles; mais elle 
sur l'île de Syra. Chap. IV. est plus fraîche et plus humide que la plupart des îles de l'Archipel. Le bourg est à un mille du port, tont autour d'une colline assez escarpéc, sur la pointe de laquelle sont situées la maison de l'évêque et l'én̆lise épiscopale, dédiée à Saint-George. "

"La principale fontaine de like est fort ancienne coule an fond l'une valléc, assez près de la ville: les gens vu pays croient, je ne sais par qu'elle tradition (voyez ce que j'en ai dit au chapitre 2. ), qu'un venoit autrefois s'y purifier avant que daller à Délos. On nous avertit trop tard qu'il y avoit une inscription à cette fontaine; il fallut profiter du vent, sans ponvir aller l'examiner. «e n'ai ancune connoissance de cette inscription, ainsi je ne peux rien dire làdessus. »

Il est constant dans tout l'Archipel, que l'ile de Srra a 36 milles de circonférence, et peut-être même 40. Son port est effectivement bon, et tiès-commode pour les frégates et des navires matrchands; mais à cause de sa petitesse, il ne peut guère contenir plus de denx ou trois valisscaux de ligne. C'ent dans la rade fornée par une île (éloignée du port diun mille environ) et le continent de Syra, 
que plusieurs gros vaisseanx, frégates et autres bâtimens, peuvent mouiller en sureté.

Le port de Syra est entre deux passages très-" importans pour les principales échelles du Levant. Situé d'un cûté entre les îles de Nègrepont et d'Andros, et de l'autre entre Tine et Mycone, il est si commode et si favorable aux vaisscaux qui éprouvent des vents contraires dans ces parages, qu’il est peu de semaines où l'on n'y voie aborder des bâtimens, et sur-tout des Francois. On y en a compté quelquefois 15 et 18 de celte seule nation. La commodité d'un tel port pour la sureté de la navigation françoise, et l'extrême altachement des Syriotes pour cette nation, qui ne s'est jamais démenti, sembleroit mériter l'attention du gouvernement pour y établir un vice-consulat. La présence diun consul du roi y seroit souvent très-nécessaire aux bâtimens françois, et dans beaucoup de circonstances elle ne seroit pas moins avantageuse aux habitans de Syra.

Le pays est frais et humide plus que la pluprart des îles de l'Archipel, comme l’a remarqué 'Toumefort : il y a plusicurs sources d'eau, et il cst couvert de puits dans toute son étendue: on pourroit y en faire un plus grand nombre, 
SUR L'île de Syra. Cha p. IV. GI

l'eau n'étant presque par-tout qu’à cinq à six preds de profondenr. Les caux y sont excellentes, et de la plus grrande salubrité, sur-tout celles de la fontaine qui fomrnit toute la ville, et d'me autre fontaine qui est à trois on quatre milles de la ville, du sommet d'une montagne qu'on appelle oúprzzas, Siningas.

Le bois n'y est pas si rare que Tournefort le dit, et il l'étoit bien moins avant les anciennes guerres des Tures et des Vénitiens. Ceux-ci détruisirent les forêts de la partie des montagnes qui regardent l'île de Délos : elles étoient couvertes d'areflies ou fidhes, que Tournefort appelle cèdres ì fenilles de cyprès. Ces arbres doment beaicoup de graines semblables au geniève, et leur bois fait un feu très-vif et très-brillant; on croit cependant que sa flamme ronge le cuivrc. Plusienrs collines vers le milieu de l'île et vers le couchant, sont encore couvertes de ces arbres. Ceux-ci ont une propriété particulière, qui est que lorsqu'on les conpe à quelques pieds de terre, pour ne pas les faire périr, il fait leur laisser quelques branches on rejetons; parce que le tronc re pousse jamais. On trouve encore à Syra, une quantité d'autres arbriseeaux, de schizio, de ligharia, dit agnus casıus, 
d'oliviers sauvages et antres, qui tous fournissent assez de bois pour la consommation des habitans. On ne s'y sert des broussailles que pour chaufier les fours. Presque toutes les maisons en ont un particulier: on n'y fait le paiu qu'une fois par semaine, le samedi. C'est alors un plaisir de sentir l'odeur de la sauge, du thym, des branches de schino et autres plantes aromatiques qu'on y brûle.

Toutes les maisons de ménage ont un moulin à bras, et ordinairement chaque famille moud son blé chez elle: le pain qu'on en fait est infinment meilleur que celui qu'on fait avec la farine des moulins à vent. Il y en a sept a huit auprès de la ville; ils sont construits en forme de tour ronde; il ny a que le dôme qui toume, le reste est immobilc. Ils ont huit voiles: du milieu de l'axe qui les contient, il sort une pièce de bois de ra pieds de long; les ailes des voiles y sont assujetties par me corde qui part de leur sommet, et va se terminer à l'extrémité de l'axe, qui, entouré de toutes ces corcles, présente au vent une espèce de pointe.

Le haut de la colline sur laquelle est bâtie a ville, est occupé par l'évêché ct la cathédrale, ainsi que le dit très-bien Tournefort, et non, 
SUR L'îlE DE Syra. CiHAP. IV. comme le prétendjM. Kléement dans ses voyages, par la maison du gouverneur turc, cependant M. Kléement quia été à Syra, ponvoit très-aisément le saroir : cela doit nous tenir en garde sur les relations de pareils royageurs.

Outre la cathédrale, il y a dans la ville sept à huit autres églises, dont deux sont desservies par les papas grecs; cependant l'une des deux appartient en propriété à une fámille catholique. Il y a en outre, tant autour de la ville que dans toutle reste de l'île, plus de cinquante autres petites églises ou chapelles. Chacune à son marguillier et son chapelain, élus au sort tous les ans. Il doit, la veille et le jour de la fête du patron de la chapelle, chanter vêpres, et célébrer l'office divin. Les paysans qui ont leurs granges dans le quartier, s'y rassemblent avec d'autres familles de la vî̉le, pour assister à la fête, qui se termine ordinairement par des réjouissances et des danses. On appelle ces fềtes Panighiria; elles font leplus grand amusement du peuple de la campagne dans la belle saison.

Toutes les productions de Syra sont excelientes: son froment est de la première qualité; l'orge y est le meilleur de l'Archipel : aussi on mange à Syra le plus beau pain des îles. Cela provient aussi du 
levain dont on se sert : nous en parlerous ailleurs. Les pois chiches de Syra sont recherchés de toutes les îles, et son coton quoique un peu rougeâtre, est employé à d'excellentes fabrications.

Dans l'immense quantité de figues que le pays produit, on en distingue de denx espèces; l'une, appelée ghlico-marona, marronia doux; et l'autre xino-marona, marronia aigres. Les premières, quand elles sont fraîches, ne valent pas grand'chose, mais sechées au soleil, et passées au four, elles sont très-bonnes. Les autres sont d'un goût exquis. Quoique Tournefort trouve nos figues sèches inférieures à celles de Provence, d'Espagne et d'Italie, les ghlicomarona de Syra, et lesminilineca de Scio méritent certainement la préféreuce.

Les figuiers y sont énormes; on en voit un entre antres dans le quartier appelé Caviri, qui a quatre-vingt-dix pas de circonférence: il donnoit, dans le temps de sa vigueur, de quinze à dix-huit charges de fignes sèches: la charge pése environ cent cinquante livres.

Les raisins n'y sont pas moins abondans; on en cultive une espèce qu'on nomme xilomacherudha blanche et noire, pour former des treillages ou des berceanx. La blanche sur- 
tout est très-délicate, et la noire donne des grap. pes qui pèsent jusqu'à douze livres. On a conupté pendant trois ans consécutifs, cent trente-quatre grappes de raisin sur une souche; et sur un antre cep de vigne planté dans un terrain trègras, on a compté jusquà quatre cent quatrevingts grappes; et l'intendant de l'évêché de notre île m’a plus d'une fois assuré qu'on avoit fait soixante-quinze bouteilles de vin, avec le raisin d'un seul cep.

Le sol de Syra est très-favorable à la production des féves. Cette île autrelois en produisoit une quantité incroyable; mais depuis plusieurs années qu'il s'y est introduit une plante pernicieuse, qu'on nomme licos, loup, à peine peut-on en manger de fraîches. Cette plante, qui, semblable à l'asperge, s'élève à dix ou douze pouces de terre, pousse un grand nombre de rejetons dans la racine; vers la floraison des féves, elle dévore leur substance, et finit par les faire périr.

Nous avons à Syra deux espèces de safran, l'une domestique, et l'autre sauvage. La premìre est la seule que uous estimons; elle vient natureliement sur nos montagnes, sur-tont dans la partie du nord de l'île. J'ai comparé à Paris notre safran arec celui d'Angleterre, et Tome $I$. 
celui que M. l'abbé Tessier a fait cultiver à Rambouillet; jai reconnu que le safran des îles du Levant donneroit cinq fois plus de teinture que ceux de France et d'Angleterre, qui, à ce que je crois, ne valent pas mieux que notre safran sauvage.

Il y a à Syra une plante très-nuisible aux produits de la terre; elle est vivace, herbacée, épineuse, portant de petites fleurs qui tirent sur le blanc et le rouge, ayant des racines fortes et profondes : nous l'appelons alonidha. Elle se mêle tellement dans les blés, et dans les orges, qu'il est très-difficile de les moissonner : les autres terrains qui ne sont pas semés en blé, en sont tellement couverts, que presque aucun légume ne peut y venir. Elle a déja gagné la belle plaine qui est auprès du port ; le reste de l'île en est menacé. On croit généralement dans le pays, que le germe en a été apporté de l'île d'Andros, dans les excrémens des bœufs venus de cette île à Syra. Quoi qu’il en soit, celui qui indiqueroit un moyen pour la destruction du licos, cet ennemi desféves, et pourcelle de l'alonidha, scroit le bienfaiteur de l'île et de ses habitans.

Cette plante qui fait verser des larmes aux Syriotes, et sortir du sang de leurs mains, lors 
de la moisson, fit grand plaisir à Tournefort, lorsqu'il l'a découvrit. Je crois en effet que notre alonidha n'est autre chose que l'alhagi maurorum, dont il nous donne une longue description dans une lettre sur l'île de Syra, et qui produit la fameuse manne de Perse. Cela m'a déterminé à rapporter ici ce qu'il nous dit dans sa lettre, sur la nature et l'utilité de cette plante. Eh! qui sait si les Syriotes, et autres insulaires, excités et instruits par cette relation, ne s'appliqueront pas à tirer quelque arantage d'une plante qui, jusqu’a présent, leur a été funeste. Voici comme s'exprime Tournefort.

"Les îles que l'on voit autour de Syra, ne sont pas assurément ces Anticyres si fameuses par leur ellébore : celles-ci sont dans le grolfe de Zéiton, au-delà de Négrepont, vis-à-vis le mont Oëta, sur lequel on prétend que mourut Hercule. Au lieu d'ellébore, nous trouvâmes dâns Syra, au bord de la mer, une plante qui nous fit beaucoup de plaisir; c'est celle qui produit la manne de Perse. Rauvolf, médecin de Hambourg; qui la découvrit dans son voyage du Levant, en I 537 , en a parlé sous le nom d'alhagi maurorum; mais il l'a décrite si succinctement, suivant la coutume de ces temps-là, que j'ai cru en devoir

$\mathrm{E}$ ij 
faire une description exacte sur les lieux, de peur qu'elle ne nous échâppat dans le reste de notre route. Il me parut même fort extraor dinaire qu'une plante, qui fait une partie des beautés des plaines d'Arménie, de Géeorgie et de Perse, se trouvât comme retranchée danles îles de Syra et de 'Tine. Wheeler l'observa dans Tine, et la prit pour une plaute non décrite. J'en ai fait ungenre particulier, sous le nom dallagi ".

"Ses racines sont ligncuses, ćpaisses de quatre ou cinq'lignes, brunes, garnies de fibres ondoyantes, peu chevelues; les tiges ont près de trois pieds de haut, sont épaisses d'environ deux lignes, vert-pâle, lisees, dures, pliantes, blanches, accompagnées de fouilles assez sémblables à celles de la renouće (polygonium lalifolium, c. в. PIN.); les phus grandes ont sept ou huit lignes de long, sur environ trois lignes de large, vert-pâle aussi, lissées, assez pointues à leur naissance, attachées par un pédicule fort. court, arrondies à l'autre bout, ou elles sons quelquefvis légèerement échancrées, et terminéct souvent par une pointe fort déliée; cette pointe n'est autre chose que l'extrémité de la côte, liaquelle traverse les feuilles sans former de 
nervure sensible: à côté des feuilles se trouve tonjours un piquant dur et ferme, long depuis cinq lignes jusqu'à un ponce, épais quelquefois d'une lignne à sa naissance, rayé dans sa lonģuenr,et roussâtre à son extrémité; les piquants des branches sont plus petits, et naissent des aisselles des feuilles; ceux par où finissent les branches et les tiges, ont un pouce et demi de long, sont plus déliés que les autres, et chargés chacun de deux ou troisfleurs légumineuses, longues d'environ demi-ponce, dont l'étendant est relevé, purpurin vers le milieu, rouge effacé sur les bords, arrondi, légèerement échancré; les ailes sont plus courtes et plus étroites, ronge-lavé, purpurines en dedans, de même que la feuille inférieure, qui est obtuse et plus large : cette feuille enveloppe une graine blanche, frangée, chargée desommets jaunâtres, et couvre un pistil long de quatre lignes, terminé par un filet; lecalice est un godet long d'une ligne et demie vert-pâle, lisse, légèrement cannelé; lorsque la fleurest passée, lepistil devient megousselongue d'environun pouce, courbéc le plus souventen faucille articulée, roussâtre, épaisse de deux liggnes, dans les endroits oì les graines sont renfermées; car les articulations sont fort étranglées et se cas- 
sent facilement: ces graines sont brunes, hautes d'une ligne, un peu plus larges, et de figure d'une petite rein : c'est la structure de la gousse qui distingue cette plante des espèces de genêt et dè ganista spartium."

"Je ne sais pas si l'alhagi donne de la manne dans les îles de Syra et de Tine, mais je sais bien que les geus du pays ignoroient que cette plante fournit une drogue qui purge si utilement. Cest principalement autour de Tauris, ville de Perse, que l'on en fait la récolte, sous le nom de Imurgibin, ou rerenjabin, rapporté dans Avicenne et dans Sérapion. Ces autcurs ont cru qu'elle tomboit sur des arbrisseaux épineux, quoiquil soit très-cerlain que éest le suc nourricier de la plante que l'on vient de ciécrire."

«Dans les grandes chaleurs, on s'apperçoit de petites gouttes de miel répandues sur les feuilles et sur les branches de cet arbrisseau; ces gouttes s’épaississent et durcissent par graines, dont les plus grosses sont du volume des graines de coriandre. On recueille celles de l'alhagi, ct on en forme des pains roussâtres tirint sur le brun, pleins de poussière et de feuilles qui en altèrent la couleur, $\hat{e} \hat{e}$ en diminuent peut-être la veru: i] sen faut bien que cette manue soit 
sibelle que celle d'Italie. On en vend de deux sortes en Perse. La plus belle, et la plus chère, est parpetites graines; l'autre est comme en pâte, et contient plus de feuilles que de manne."

Finissons cet article par tracer le portrait des habitans de Syra. Sous le ciel le plus beau, sous le climat le plus pur et le plus sain, au milieu de toutes les richesses de la nature, l'espèce humaine ne doit pas être la moins parfaite de ses productions. Aussi voit-on à Syra des hommes généralement bien faits, robustes, agiles et courageux. Les femmes que les travaux de la campagne n'ont ni déformées, ni épuisées; celles à qui une fortune aisée permis de mener une vie douce et tranquille, ou même d'habiter quelque temps à Constantinople ou à Smyrne; celles-là sont belles, comme toutes les femmes du Levant, et c'est en faire un assez grand éloge. 


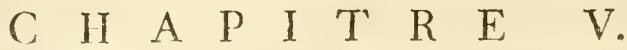

$D u$ gousernement temporel et spirituel de Syra.

PARlons maintenant du gouvernement de Syra. Cette íle, comme le resie des Cyclades, est soumise aux Tuics. Je n’ai pas besoin de dire que, dans des temps reculés, elle a changé plusieurs fois de maitre; toutes les parties du globe habité ont éprouvé le même sort.

Syra, après avoir obéi successivement aux Grecs, aux Romains, aux empereurs de l'Orient, reconnoissoit, lors de l'invasion des Otto: mans, les dics de Naxie, dont l'origine monte aux temps des croisades, comme nous l'apprend Flavius Blondus, conp. rer. Ventet, et comme nous l'avons rapporté ci-dessus, à l'article premier.

Quoique dans la liste, que nous y arons donnée des îles sujettes aux ducs de Naxie, on n'en compte que dix, et que le nom de Syra n'y 
sur L'île de Syra. Chap. V.

soit pas compris, c'est cependant une tradition constantequ'elle leur a appartenu, au moins daus la suite arec onze antres; et que c'est pour cela que ces îles, désignnées d'abord toutes ensemble par le nom de Dhucanisa, iles du Dac, furent appelées depuis par corruption Dhodhecanisa,les douze iles. On cite même deux anecdotes, qui pourroient servir à appuyer cette opinion, mais que je me dispense de rapporter ici, pour ne pas paroître vouloir fonder mes assertions sur des bruits populaires.

Depuis la conquête des Ottomaus, tantôt Syra releva immédiatement des Grands-Seigneurs, et alors l'épirropos de Syra étuit obligaé de porter, avec les épitropes des autres îles, son tribut au capitan jacha à Drio; tantôt elle dépendit des seigneurs particuliers, auxquels lesgrandsseiçneurs avoient abandonné la propriété. Sultan Abdul-Amid, le dernier empereur, ayant fait couperlatête, ily a donze à treize ans, au Seignneur de Syra, a donné en apanage cette île ainsi que celle d’Andros, à une Sultane sa nièce, sœur du sultan Sélim régnant. Cette princesse a pris à cour sa nouvelle possession; elle s'est montrée protectrice zèlée de ses sujets. Il faut pourtant observer qu'elle ne touche que la moi- 


\section{PRECIS HISTORIQUE}

tié du produit du tribut de l'île. L'autre partie est affectée à l'entretien de la garnison de la forteresse de Chothim où de Bender.

Les droits annuels perçus sur l'île de Syra, y compris les dépenses ordinaires de la communauté, sont de 7 à 8000 piastres. Ces droits sont assis, partie sur les terres, partie en capitation, et partie en taxe arbitraire.

Les droits sur les terres, dès le commencecement que Syra subit le joug des Ottomans, ont été taxés à 500 piastres. Voici comment cela c'est exécuté ; des commissaires envoyés par la Porte parcoururent toute l'île avec des experts, et à mesure ils taxoient les propriétés de chaque particulier de deux liards, de cinq, de dix, de quinze, etc. c'est-à-dire, une aspre, deux aspres, etc. (comme les Grecs les appellent ), à raison de leur étendue, de leur qualité, et de leur proximité de la ville. Tous ces liards ou aspres ensemble, forment 500 piastres. Il faut savoir que, dans le cours ordinaire du commerce, une piastre vaut 120 aspres; mais dans le payement du tribut, la piastre ne vaut que roo aspres, ou, ce qui revient au même, pour ıoo aspres, on paye une piastre. Ce droit se 
nomme en grec dhosimo; on l'appelle encore charalsi, qui est un mot turc.

Dans les commencemens, Syra ne payoit que ces 5oo piastres; ainsi les terres en particulier ne payoient que ce qu'elles étoient taxées. A mesure ensuite que les tributs doubloient ou triploient, les taxes des terres doubloient et triploient aussi. Actuellement que le tribut de l'île monte à 15 ou 16 bourses, c'est-à-dire à 8000 piastres, ces taxes sont montées à neuf; de sorte qu'un terrain, par exemple, qui payoit originairement une aspres, en paye neuf aujourd'hui; ce qui forme 4500 piastres, ou neuf bourses, la bonrse faisant 500 piastres.

Le restant du tribut pour monter à la somme totale, est assis, comme nous l'avons dit, sur la capitation et la taxe arhitraire. Tous les mâles âgés de treize ans environ, sont sujets à cette capitation. Les chefs de famille, c'est-à-dire, tous les hommes mariés, payent quatre piastres (on fait grace de quelque chose aux jeunes mariés); les garçons et les ecclésiastiques, moitié. Quand un chef de famille meurt, son fils, s'il en laisse, de quelque àge qu’il soit, est censé le chef, et paye capitation entiere. Outre cet impôt, il y a encore unc pretite imposition 
d'environ quinze sols, à laquelle sont sommis les seuls chef's de famille. Les Tures l'appellent spendja, du mot italien Spesa, dépense. Ces deux arlicles réunis montent à 2500 piastres.

Enfin, par la taxe arbitraire, on remplit la somme totale. A cette taxe sont sommises toutes les personnes tenant ménage, ou faisant quelque profession : les ecclésiastiques en sont exempts, excepté lorsqu'il y a quelque somme extraordinaire à payer par la communauté, et alors ils contribuent librement. La taxe arbitraire est ordonnée et répartic par une commission nommée par la communauté : elle est ordinairement composée de vieillards, et de personnes de différentes professious, ayant toujours à leur tête l'épitrope. Les cahiers de toutes ces impositions étant faits, c'est à l'épitrope de les faire lever, et d'en faire passer l'argent à Constantinople, au procureur de lîíc de Syra, qui le remet sur-le-champ au trésorier de la Sultane.

A en juger par la forme du gouvernement établi à Syra, et dans presque toutes les autres iles, on croiroit que ce sont autant de petites républiques tributaires. Toute l'autorité exécu- 
SUR L'îlE DE SYR A. CHAP. V.

trice réside dans les mains de l'épitropos. C'est un mayistrat annuel, qui garde quelquefois sa place deux, trois ans, et même davantagge s'il en est digne, ou s'il est assez intrigant pour savoir s'y maintenir. Il a pour conseil les proestus, qui sont les anciens épitropes. L'épilropos ne peut rien innover; il est obligé de s'en tenir aux coutumes du pays consignées dans les capitulaires, ou le code de lois par lequel la comnunauté est régie depuis un temps immémorial. Nous citerons, un de ces capitulaires qui concerne la vente des biens. Un particulier quelconque, qui a résolu de se défaire du sien, n’est pas toujours libre de le vendre à qui il lui plaît. Les plus proches parens, d'où ce bien lescend, ensuite les voisins qui touchent à ce même bien, ont de droit la préférence : le vendeur fait dabord estimer son bien par les experts; ensuite il en fait annoncer la vente dans toute la ville, par un crieur public; et si, dans l'espace de 15 jours pour ceux qui se trouvent clans l'ile, et d'un an pour ceux qui sont en pays étranger, personne de ceux qui ont des droits ne se présente à la clancellerie, alors le propriétaire rst le maître de le donner à quiil lui plaît, et au prix qu'il veut. Je dis au prix qu'il veut, car 
quand ce sont les parens, il n'en peut exiger que le taux de l'estimation.

Cette loi, au premier coup.d'œil, senble injuste; mais ce qu'on peut y trouver de déraisonnable disparoîtra, si on fait attention qu'à Syra, les terres sont partagées à-peu-près également entre toutes les familles; cette égalité dans les propriétés contribue aussi à entretenir celle des fortunes entre les cituyens. Cette loi a été établie pour arrêter ou gêner les atteintes quon pourroit porter à un équilibre si précieux.

Il y a une clause que l'on insère ordinairement dans les contrats de mariage; c'est qu'en casque les contractans viendroient à mourir saus enfans, leurs biens immeubles patrimoniaux retourneront à leurs parens; mais avec la liberté au testateur d'avantager un ou plusieurs d'entre eux, et sous la condition de laisser quelque chose aux autres héritiers. Par cette clause personne ne peut donner ses biens patrimoniaux aux main-mortables. Il y a cependant une loi qui porte, que si le testateur a fait des acquisitions, il pourra en disposer en faveur de qui il voudra; et qu'à défaut de biens acquis, il lui sera libre de disposer encore du dixième des biens 

qu'il a apporté en se mariant, pour faire prier pour le repos de son ame.

Au reste, quoique nous ayons dit que l'épitropos, assisté des 'proesti, juge les différends entre les particuliers, cependant, quand il survient quelque contestation au sujet des testamens et des contrats de mariage, c'est ordinairement à l'évêque que la connoissance en est réservée, tant parce que de pareilles discussions exigent plus de capacité, que pour tâcher de les terminer à l'amiable et sans frais, et empêcher que la chose ne soit portée devant le cadi, qui exige ro pour roo pour ses honoraires de toutes les affaires qu'il juge. Il faut observer ici que chez les Turcs, celui qui gagne son procès paye les frais: cela se pratique ainsi avec tous les juges, depuis les cadiaschers, jusqu'aux simples cadis. Ce n'est qu'au tribunal suprême de la porte, qu'on nomme en turc pacha-capichi, qui répond ici à conseil, que les procès se jugent sans frais. Par cet arrangement du gouvernement turc on voit que, si d'un côté le malheureux qui a été condamné à perdre son procès n’est pas ruiné par le payement des frais, il reste cependant continuellement une porte ouverte aux méchans, pour chercher 
chicane aw riches, ce qui procure une source intarrissable de revenu aux juges. Ces frais , comme nous venons de le dire, sont les dix pour cent de tonte la valeur en question; de sorte que si l'on plaide devant un cadis, sur la validité d'un testament de la valeur de trente mille livres, celui qui gagne son procès, est obligée d'en payer trois mille. Quvique les cadis ayent le droit de s'attribuer le jugement de tous les différends des particuliers, cependant les ordres du Grand-Seicineur portent, quils ne peuvent juger que de concert avec l'épilropos et les proesti, qui doivent signer leur nom au bas de toutes les sentences, et selon les risages et les capitulaires du pays; mais comme on voit à Syra peu de procès, grace aux bous réglemens dont nous avons parlé, le cadi reste communément à Andros, où il trouve mieux son compte; et quand il vient à Syra, ce qui est très-rare, la communauté s'en délivre ordinairement en lui payant trente piastres.

Quant à l'avantage d'être soumis plutôt atıx sultanes qu'au Grand-Seigneur, ou au capitanpacha, il y en a infiniment de l'être aux sultanes, et sur-tont lorsqu'elles ont de l'énergie ou du caractère. Les vexations sont plus rares 
sur l'île de Syra. Chap. V.

et moins accablantes. Leur protection a beaucoup d'influcnce dans plusieurs circonstances. Les Syriotes s'en ressentent à Constantinople et ailleurs; ils sont exempts de capitation moyennant une déclaration quion appelle tescheré, et qui leur est fournie par l'Intendant de la Sultane; c’est une espèce de quittance de la capitation paycée à cette Princesse. Tout habitant de Syra quis'expatrie, doit avoir une de ces lescherés, pour l'exhiber à ceux qui perçoivent la capitation sur les sujets du Grand-Seigneur. Un autre avantage, c'est que Syra, soumise à la Sultane, est libre d'accepter ou non un Voivoda pour lever ses impôts. Ces Voivodas sont des espèces de fermiers qui payent à Constantinople, et d'avance au Capitan-Pacha, au moins la moitié des tributs de l'annéc d'une île entière. Au moyen de ce payement, ilssont autorisés à faire la levée de l'impôt, et les îles soumises au Capitan-Pacha sont ordinairement forcées de recevoir ces exacteurs qui désolent les habitans de la campagne, et exercent sur les peuples toutes sortes de vexations.

Syra n'est point obligée encore de faire des présens, ni au Capitan-Pacha, ni à son Dragoman, ni aux autres subalternes, comme cela se pra

\section{Tome $I$.}



tique dans les autres îles qui sont encore assujetties à des oppressions d'un autre genre. Le Capitan-Pacha a le druit-d'expédier par mer des courriers appetés en ture mensil, pour des commissions particulières, à la charge des îles par où ils passent. Ces mensils sont souvent des aventuriers, ou des escrocs, qui, moyennant quelque somme, se font autoriser par des Officiers subalternes, sous différens prétextes, et qui yont d'île en île pour extorquer quelque argent. D'abord on est obligé de les nourrir pendant quelques jours; et malheur à leurs hôtes sile temps cievient mauvais; il fant alors les nournir peindant des semaiues entières eux et leurs équipages, leur payer le trajet jusqu’à une autre île, et leur faire encore présent de quelques piastres par dessus le marché.

Quant au gouvernement spirituel de lile, Tournefort, qui a été cité dans l'article précédent, a raison de cire quill n'y en a pas de plus catholique dans tout l'Archipel. En efiet, pour une poignée de Grees, on y compte près de qaatre milie catholiques du rit latin. Si l'on est curieux de savoir comment il est arrivé qu’au milien de tant d'iles habitées par des pemies de religion 
stir L'île de Syra. Char. V. 83 grrecque, Syra s'est trourée d'un rit différent, on n'a qu“a jeter les yeux sur les familles européennes qui étoient sur le continent, lorsque les Seigneurs Francois et T'éniticns y aroient de petites sourerainetés. Les Turcs les ayant forcés de les abandonner, plusieurs d'entre elles se seront réfugites à Syra. En eflet, les trois quarts au moins des noms de famille de Syra sont européens, tels que les Damiraglia, les Roussaus, les Rossi, les Cappello, Cappeila, Damolli, Della Rocea, Dapola, de la Croix, la Picrre, Marinelli, Brinclesi, Dalegio, Durazzo , Privilegio, Yacondio, et plusieurs autres. On ne peut attribuer à ces noms, ni un caractère grec, ni une origine greçue. On prétend quine peste des plus mentrieres ayant emporté à Syra presque tous les habitans, et que plusieurs filles ayant été épargnées, les officiers et autres personnes de la flotte rénitienne s'y étabiirent, $\because$ marierent, et apportèrent dans l'île la religion et le rit romain, qui s'y est depuis toujours conservé et propascé.

Un Evèque préside au gouvernement spirituel de Srra; il est toujours nommé par liome, et suus la protection du Roi de France, sans laquelle il ne pourroit peut-être pas subsister. 
Outre la nomination de Rome, nos Évêques, pour prendre possession de leur diocèse, et pouvoir exercer librement leurs fonctions, ont besoin d'être autorisés par un barat de la Porte: ce barat donné aux Évêques, n'est autre chose, qu'une espèce de pouvoir que les Papes ou les Patriarches de Constantinople accordent dans leurs bulles, ou dans leurspandachus, aux Évêques de leur communion.

Lorsque les Ottomans soumirent l'Empire des Grecs, et s'emparèrent de Constantinople, ils eurent la bonne politique de vouloir retenir les Grecs dins les pays conquis, et deles empêcher de s'expatrier. Le moyen le plus propre dont se servirent les Empereurs ottomans pour y parvenir, fut de leur accorder la liberté de religion, de reconnoître leurs pasteurs, et de leur laisser presque tous les priviléges dont ils jouissoient avant la conquête. C'est par une suite de cette faveur que la Porte a imité les bulles on pandachus ( où sont contenus les pouvoirs spirituels des Évêques ), et même quelques ordonnances des Empereurs grecs, sans lesquelles ces Évêques ne powvoient aller prendre possession de leurs sièges. Ces barats sont ordinairement adressés aux Gouverneurs des Provinces 
ou des villes. Les objets principaux qu'ils contiennent, sont, $\mathbf{I}^{\circ}$. de gouverner tous les sujets chrétiens de leur nation, demeurant dans le district de leur diocèse, pour tout ce qui a rapport à la religion; $2^{\circ}$. de percevoir les dimes et autres droits qui sont en usage parmi les chrétiens; $3^{\circ}$. de juger les affaires civiles de leur Clergé. En vertu de ce privilège, un Cadi, par exemple, à Syra, ne pourroit appeler en juscice un Ecclésiastique quelconque, ni une Religieuse, sans la permission de l’Évêque.

Il y a d'autres barats que la Porte est en usage d'accorder aux Ambassadeurs des Cours européennes qui résident à Constantinople., en faveur de différens sujets de l'Empire Ottoman. Par le moyen deceux-ci, les sujets du Grand-Seigneur qui les ont obtenus, sont regardés comme faisant corps avec la nation de l'Ambassadeur, à la demande diqquel ils ont été accordés, et ils juuissent des mêmes privilèges et des mêmes franchises.

On doit s'attendre que M. le chevalier de Monradgea, dans son excellen Tablean de l'Empire Ottoman, nous expliquera dans le plus ģrand détail, l'avantage de ces Barats, qui a'est pas le mềme pour tous les Evêques; car il $\mathrm{y}$ a des 
Siènes parmi less Gres, à qui la Porte a accordé um plus grand nombre de privileges et de pourvoirs qu'aus antres.

C'est l'Ambassadeur de France à Constantinople, qui demande à la Porte le barat pour lÉvêque de Syra. Ce barat coûte 40 piastres pour chaque nouvel Evêque, et parcille somme à lavinement au trône de chacute nouvean Siltan, pour le renouveler. L'Ambassadew de France, on la nation, en fait présent ordinairement aux Evéques de Syra : il est encore d'uage, lorsque ces Evêques sont sacrés a Constantinople, dans la Chapelle de ect "Ambassadeur, que celui-ci, et la nation, lui cont présent, ou d'une croix, oud'une bagne, ou de quelque antre ornement épiscopal.

Les revenus de l'Evêque de Syra sont trèsbornés; à peine montent-ils à 400 piastres, en y comptant cent piastres qui lui sont données par la caisse de la marine de France, et pareile somme, par la cour de Rome. C'est le plus panvre de tons les Evêques de l'Archipel, quoique les revenus de ceux-ci naillent pas à mille prastres.

Le nom de l'Evirue actuel de Syra, est Tho- 
SUR L'île de Syra. Char. T. 87 mas Funton. Ce Prélat est d'une familie dislinģnće; dejuis plusieurs grénćrations, ses jarens sont à Coustantinople, au service de France, en qualité d'interprètes du Roi à la Porte. Son Aieul, gentilhomme de Valence en Dauphiné, fut le premicr qui s'y établit sous l'ambassade de M. de Gérardin, sur la fin du demier siècle.

Il y a cncore à Syra quatre Prêtres, espèce de Chapelains, qui, sous l'inspection de l'Evêque, desservent la Paroisse, charun a leur tomr, toutes les semaines. Le casuel de ces bons Ecclésiastiques est si modique, qu'il ne va pas à dix piastres par an; cependant rien n'est si respectable que l'attention qu'ils apportent au service spirituel de leurs paroissiens. IVais ce qui devroit servir d'exemple à beaucony de Prêtres en Europe, c'est l'assistance et les soins infatigables qu'ils donnent aux mourans. Dìs qu'un malade a été administré, ils ne l'abandomnent ni le jour, ni la nuit: on les a vus souvent le garder pendant huit jours, et ne preadre quelque repos que sur lies planches. $\Lambda$ Syra les Prêtres ne gardent pas les morts; ils sont persuadés que rien ne les roublera désormajs, et qu'ils n'ont pas besoin de compagnie; dailleurs Dienentend 
les 'prières de loin comme de près. Ce n'est qu'au moment desa mort, qu'ils croient qu'un Chrétien a besoin d'assistance, de conseil et de secours spirituels.

Le clergé de Syra est assez nombreux : on y compte de 40 à 50 ecclésiastiques, qui sont tous ordonnés ad litulum patrimonii. Il y en a parmi eux qui ont fait leurs études à Rome, ou en d'autres parties de la chrétienté. Plusieurs d'entre eux sont employés à l'éducation de la jeunesse; ils lui apprennent la lecture, l'écriture et les premiers élémens de la religion. Dans presque toutes les échelles du Levaut, où il ya un consul de France, on trouve un prêtre de Syra pour aumônier. Outre les vingt-cinq Religieuses Capucines, du tiers-ordre de St. François, dont parle Tourncfort, dirigées par les Capucins, il y a à Syra à-peu-près le même nombre de Jacobines, sous l'inspection immédiate de l'ordinaire. Deux Religieuses Ursulines s'y occupent de l'éducation des filles. Ces Religieuses, qui ne sont pas cloîtrées, mènent une vie très-exemplaire dans les maisons de leurs père et mère, ou d'antres très-proches parens.

Placée au milieu d'un chaos d'erreurs, Syra ne le cède pas aux pays placés au centre 
sur l'île de Syra. Chap. V. 89 de la Chrétienté, pour tout ce qui est relatif au spirituel. C'est une agréable surprise pour ceux qui ne connoissent le Levant qu'imparfaitement, de voir, quand ils abordent à Syra, la liberté avec laquelle on excerce publiquement la religion catholique, et sur-tout dans la procession de la Fête-Dieu. Le jour de cette auguste cérémonie, les vaisseaux de toutes les puissances chrétiennes qui se trouvent dans le port, font flotter leur pavillon, et saluent la procession de plusieurs coups de canon. Cette douce liberté dent Syra jouit, est le fruit cle la protection du Roi de France auprès de la Porte. 


\section{C $\mathrm{H} A \mathrm{~A}$ I $\mathrm{T}$ R E VI.}

Prolection du Roi de France, envers les habilans de i'ile de Syra, relalivemeni à leur religion, etreconnoissance de ceñ̄-ci enuers les Frangois.

Tous les catholiques répandus dans l'Empire Ottoman, recomnoissent le Roi de France pour leur protecteur; ct celte protection s'étend particulièrement sur ceux qui suivent le rit latin; les habitans de lîle de Syra en particulier en ont de tout temps ressenti les heureur effets.

On évalue communément le nombre de ceus qui, dans les états du Grand-Seigneur, suivent la religion catholique, à trois millions, sous dlférens rits, clont partie a adopté le rit greec; nais les Syriotes suivent le rit latin, et c'est la conformité de culte qui les tient plus paruculierement attachés à la France. C'est sans cbute cet attachement qui leur a mérité de la pat de 
SUR I'île de Syra. Chat. VI. gr ceite nation une protection toute particuliere, a 1 mojen de laquelic ils jouissent clans leur île d'une a:sez grande liberté pour l'exercice de leur relicion.

Nous arons déja remarqué au chapitre précéclent, que nos Érêques, sous les auspices des Rois de France, jouissoient d'une paisible tranquillité dans l'exercice de leur ministère. Lonis XIII a le premier fondé dans l'ile de Syra une mission de Capucins. Louis XV a protégé celle des Jésuites. Les Prêtres de SaintLazare ont succédé à ces derniers dans presque toutes les missions dont ils étoient chargés. Le zicle infatigable avec lequel ils remplissent les functions de l'apostolat, sembloit faire espérer aux Syriotes quils viendroient affermir par Jeurs instructions, des peuples environnés d'irfideles, dans le chemin de la foi, et les édifier par leurs exemples. Sans donte que des circonstances particulières ont retardé jusqu'i ce moment un ouvragge anssi bien commencé, et dont les Lazaristes doirent se promettre les plus grands succes.

La reconnoissance des Syriotes envers le Roi de France se manifeste a Syra de la maniere la plus éclatante; on pourroit mêmedire la plus 
incroyable ( $\mathrm{r}$ ). Quoi de plus singulier en effet que d'entendre au milieu des peuples livrés à l'erreur, et d'autres ennemis capitaux du nom chrétien, dans les Églises et les Chapelles particulières, retentir au loin le chant mélodieux du Domine salvum fac regem? Et pour quon ne puisse se méprendre sur le nom de celui qui est l'objet de cette prière, on y ajoute ces mots nostrum Ludoricum. Quel hommage que celui d'un peuple soumis à une domination étrangère, qui, par amour, choisit pour objet de son affection particuliere, un Roi que son attachement au seul culte catholique a fait nommer universellement très-chrétien ! Un étranger qui arriveroit à Syra dans ces instans, ne pourroit douter que la religion dominante du pays ne soit la catholique.

La publicité de ces prières et l'éclat de ces preuves de recomnoissance, ont attiré aux Syriotes, auprès de la Porte, de la part des schismatiques, le reproche de recounoître le Roi de France pour leur Sonverain, mais par égard

(1) Je puis avancer, sans crainte d'être démenti, que cet attachement des Syriotes à Sa Majesté très-chrétienne, est si profond, qu'aucun d'eux n'hésiteroit de mounir pour sa gloire et pour son service. 
SUR L'île de Syra. Chap. VI. 93 pour la France, le gouvernement a rejeté cette accusation ; d'ailleurs le mépris que les $\mathrm{Mu}$ sulmans ont pour toute autre religion que la leur, et le peu de mérite qu'ils attachent aux prières qui ne sont pas adressées à Dicu par eux-mêa mes, semble motiver cette indifférence (1).

(1) Une anecdote assez singulière peut prouver le mépris que les Turcs ont pour les prieres des chrétiens. 11 y aquelques années, que dans une province de l'Asie mineure voisine de Smyrne, on éprouva une sécheresse affreuse. Les moissons périssoient sur pied, et tout annonçoit une disette extrême. Les Turcs se rendoient en foule aux mosquées, pour obtenir de la clémence de Dieu la pluie qui leur étoit si nécessaire. Mais la sécheresse augmentant de plus en plus, il fut ordonné aux chrétiens de joindre leurs prières à celles des Turcs. Les chrétiens firent des prières publiques; bientôt une pluie abondante fit rcverdir les campagnes, et dissipa les alarmes. Aux cris de lalégresse universelle, succédèrent des murmures contre les chrétiens, dont on présumoit que les prières avoient obtenu le soulagement si desiré, et on étoit surpris qu'après tant de prières faites par les Turcs inutilement, celles des chrétiens eussent fléchi la colère de Dieu. Voici de quelle manière l'Imam trouva moyen de calmer les esprits. Vos prières, dit-il, aux Musulmans, sont auprès de Dieu comme un parfum délicieux : elles lui sontsi agréables; 
Les Syriotes se sont empressés dans toutes les occasions de donner aux François tous les secours qui étoient en leur pouvoir : nous ne pouvons en donner une preuve plus authentique, qu'en rapportant ci-après les certificats de plusieurs Négocians de Marseille, qui ont résidé dans le Levant, et des Capitaines des Navires qui fréquentent ces mers. Les originaux de ces certificats envoyés au Ministre de la marine du Roi, ont été déposés dans les bureanx de ce département.

Lorsquin marire fançois est attaqué de la peste, et quill se trouve à portée de Syra, il ne manque pas dy aborder de préférence, étant sûr d'y trowrer tous les secours possibles. On dresse des tentes dans une petite

que pour en respirc: plus long-temps la vapeur, il ne reut pas s'empresser de les cxaueer. Au contraire, lespricres des chrétiens sont pour lui conme un cadavre infect; c'est pour cela que dès qu'ils lui demandent quelque grâee, il se dépeche de la leur accorder, pour se delivrer d'une odeur qui lui est infiniment désagréable. On ne sait de quni sétomer le plus, on de l'arlresse de l'Imam qui donue unc parellye roponse, ou de la stupidite du peuple qui s'en contente. 
SUR L'île de Syra. Chap. VI. $9^{5}$ ile, éloigncé du port d'environ un mille; on fait descendre tout l'équipagge, et on sćpare les malades de ceux qui ne le sont pas. Ceux des Syriotes qui ont eu la peste à Constantinople ou ailleurs, et qui ne la craignent plus, se votrent au service des malades. D'autres vont nettoyer le vaisseau, mettre à l'air les marchandises, et dissiper le levain pestilentiel. Les Ecclésiastiques s'empressent à prodiguer tous les secours spirituels à ceux dont la vie est en danger; et après un séjour de quarante à cinquante jours, l'équipage remet à la voile, en bénissant Dieu de lui avoir procuré un si précieux hospice sous un ciel étranger, et vouant à des. hôtes si humains, une reconnoissance éternelle.

Si à la vue de Syra, un vaisseau françois est poursuivi par quelques corsaires ou pirates, les luabitans volent à son secours, ainsi que l'attestent les certificats que nous rapporterons. MM. deVergennes et de Saint-Priest, Ambassadeurs de France a la Porte, ne l'out jas ignoré. La défense que les Syriotes prirent en 1770 , d'un vaisscau francois, lorsque la flotte russe occupoit: l'Archipel, leur fut très-funeste : à l'instant où il entroit dans le port de Syra, denx petits. corsaires remplis de Stakiotes, sous pavillon 
russe y abordèrent aussi. Le Capitaine françois, accompagné du sieur Perretier, négociant, qui alloient à Smyrne, montèrent à la ville. Les Sfahiotes résolurent de s'emparer du vaisseau; mais pour y parrenir plus surcment, its formèrent le dessein d'arrêter le Capitaine et son compagnnon, à l'instant où ils descendroient de la ville. Un Syriote informé de ce complot, avertit sur-le-champ le Capitaine et l'Epitropos qui se tinrent sur leurs gardes. On sonna le tocsin, et au même instant plus de soixante jeunes gens prirent les armes et environnèrent les deux Francois. Ils les conduisirent jusqu'à bord de leur vaisseau, sur lequel ils passèrent toute la nuit, de peur d'attaque ou de surprise. Les François ayant mis à la voile le lendemain, les Syriotes voulurent les accompagner jusqu'au détroit qui est entre Tine et Mycone, et ne se jeterent dans leurs barques pour regagner le port, que quand le vaisseau françois eut assez pris le large pour être à l'abri de la poursuite des corsaires.

Piqués de se voir ainsi arracher une proie sur laquelle ils avoient compté, les pirates re. vinrent quelques jours après áu nombre de quatre à cinq cents, pow faire une descente dans l'île et piller la ville; ils se répandirent dans les différens 
SUR L'île DE Syra. CHa P: VI.

différens quartiers, et y passèrent la nuit. Les $S_{y}$. riotes se doutant de leur dessein, prirent les ar. mes aux nombre de vingt-cinq à trente, ayant à leur tête George Kxanthaki, renommé par sa valeur. La plupart des labitans étoient à la came pagne; mais malgré l'inégalité de forces, les Syriotes firent de tels efiorts de courage, combinèrent si bien leurs attaques, que les Sfahiotes et les Albanois, saisis de peur, et ne pourant juger du nombre, quittèrent bientôt la parties et regagnèrent leurs vaisseaux à toutes jambes, Une poignée de Syriotes, dont partie n'avoit pas même d'armes à feu, osa les attaquer en pleine campayne; et quoiqu'ils se battissent retranchés derrière des murs peu élevés, plusienrs d'entre eux furent tués ou blessés, et aucun des Syriotes ne reçut la moindre blessure.

Des officiers russes qui avoient été témoins de ce combat, du vaisseau qu'ils montoient, rendirent compte au général Orlof de cet acte de bravoure et de patriotisme, en lui protestant qu'ils préféreroient commander une compagnie de braves Syriotes, à des régimens entiers d'Albanois.

Quoique les Sfaliotes et les Albanois aient ćté repoussés plusieurs fois avec perte, ils n'en sont

Tome $I$. 
pas muins acharnés à la vengeance: ils attaquent sans cesse les habitans de Syra. Ce sont, de temps en temps, de vouvelles incursions, de nouvelles descentes dans des endroits ćcartés, où ils mettent tout an pillage, ravageant les campagnes, brûlant les vignes et les arbres fruitiers, empor. tant les troupedux répandus dans leschamps, et se vengeant ainsi par des traits de barbarie, de la défense courageuse et légitime que les Syriotes ont opposée à leur brigandage, et des secours quiils ont donnés au pavillon françois.

C'est sur-tout dans le cas où les vaisseaux françois font nauliage à la vue de l'île de Syra, que les Syriotes font éclater le zèle le plus ardent. Un des plus afrieux naufrages quils aient essuyés est celui de 1778. La muit de Nö̈l, par un froid excessif, un ouragan fit périr dans le yort même, un vaisseau fiançois du port de Marseille, commandé par le capitaine Girard, qui y étoit à l'ancre. A l'entrée de la nuit, la mer étoit parfaitement calme et le ciel étoit couvert. Le capitaine ordonna an Pilote de gonverner sur l'ancre, c'est-à-dire, de se mettre à ;ic, dans le dessein d'appareiller dans la nuit, dies yue le vent seroit favorable. Tout l'équipagè ćsoit livré au sommeil. Le vaisseau, après avoir 
SUR L'île de Syra. Chap. VI.

fait plusieurs révolutions autour de l'ancre (ce qu'en terme de marine on appelle jouer sur son ancre), le cable en accrocha une des pointes et lui fit quitter terre. Au point dujour, il s'éleva un vent violent de nord-est, qui obligea le Capitaine de faire lâcher le cable, sans prendre garde si le vaisseau étoit amarré, ou jonoit sur son ancre: le vent augmentant de plusen plus, le vaisseau dériva sans qu'on s'en apperçût, et fut jeté sur des rochers à quinze pas tout au plus du rivage. Le Capitaine, averti trop tard 'du danger qu'il couroit,et pour empêcher que les gens de l'équipage ne se jetassent dans la chaloupe, et n'abandonmassent le vaisseau, compa lui-même la corde, et fut, sans le vouloir, cause de sá perte et de celle d'une grande partie de l'équipage.

Au point du jour, à l'instant où tout le monde étoit rassemblé dans l'église pour' cntendre la Messe, un Missionnaire (c'étuit le Père Roxa, ex-Jésuite Napolitain, et originaire Espagnol), alloit commencer son sermon de mission aú peuple, lorsqu'on vint l'avertir qu'un vaisseau françois et tout son équipage étoient près de périr. Ce Missionnaire adresse sur-lechamp au peuple ces paroles: "Mes enfans, " des Francois, nos frères, se trouvent en ce 
« moment dans le plus grand danger : nous ne «pouvons célébrer cette solennité d'une ma"nière qui soit plus agréable à Dieu, qu'en "volant a lcur secours. " A peine eut-il dit ces mots, que chacun, animć du même zèle, court au rivage. En un instant, l'Egglise fut déserte. C'étoit un spectacle vraiment attendrissant, de voir l'empressement avec lequel les hommes, les femmes, les enfans, les vieillards même, couroient au rivage. Les uns portoient du bouillon, d'autres des liqueurs, cerax-ci des vêtemens, ceux-là des médicamens; ct ce qui rendoit cette scène encore plus touchante, cétoit la rigueur de la saison. La grêle, la neige, le vent et le froid étoient cxcessifs; à peine pouvait-on se soutenir. J'étois moi-mêne témoin oculaire de tout ce que la charité inspiroil aux Syriotes en faveur de ces malheureux. L'empressement de chacun à imaginer pour les sauver, des moyens que la fureur de la mer pouvoit faire regarder comme inutiles, rendoit encore plus aflicux le silence, qui régnoit dans ce moment. On eût dit qu'un chef habile ccmmandoit cette troupe, tant lour concert étoit unanime. Monté sur une éminence, les consolations que j'adressois à ces malheureux, redou- 
SUR L'île de Syra. Chap. VI. Ior bloient le zèle desuns et le courage des autres: moi-même jesanglottois, et je perdois à chaque instant le courage que je tâchois d'inspirer.

Le vaisseau ayant rencontré sur le rivage des rochers aplatis, y étoit tombé sur le côté, et le poids des mâts le faisoit pencher vers la mer; de manière qu'on vọoit de terre toute la quille, et qu'ainsi il n'étoit pas possible d'établir de communication pour aller porter du secours à ceux qui étoient dans le vaisseau.

On tenta d'établir un pont pour aller à bord. Pour y réussir, plus de deux cents personnes transportèrent un mât qui étoit resté sur l'île, des débris d'un autre vaisseau françois, qui, quelques mois avant, avoit péri sur le même rivage, et dont l'équipage avoit dû son salut au secours des Syriotes. On fit les plus grands efforts pour le pousser à bras jusque sur le vaisseau; mais ce fut inutilement. Quoique ce bâtiment ne fût pas à plus de quinze pas du rivage, les vagues étoient si fortes, qu'elles couvroient le vaisseau, et dérangeoient tout ce qu'on tentoit d'établir. A chaque instant on étoit contraint de quitter le travail, et à se retirer à plus de dix pas sur la terre. Le mât qu'on avoit apporté, fut mis en piëces contre les rochers. 
On chercha, par le moyen d'unc fronde, à faire parvenir sur le vaisseau une ícelle, qui, une fois saisie par cenx qui étuient dedans, auroit servi à tirer à eux une forté corde, par le secours de laquelle on espéroit qu'ils auroient pu se sauver; mais la force du vent rendit encore cette tentative inutile. On peut juger par touts ces détails, de la violence de l'ouragan.

Désespérés de voir tous leurs efforts inutiles, les Syriotes poussèrent le courage jusqu'à proposer de se jeter à la nage, pour aller saisir le bout d'un cordage, le ramener à terre, et sauver ainsi tous cenx qui auroientassez de force pour s'en servir. Cette ressource étoit, comme on voit,infiniment périlleuse ; mais, malgré le danger extrême, un habitant se jette à la mer, arrive avec des peines incroyables près du vais.seau, et crie arx matelots de lui jeter le bout d'un cable. Ceux-ci, soit que l'horreur diuc mort certaine leur eût fait perdre la tête, ou quo le bruit d'un vent violent les empêchát d'entendre, ne jetrent point la corde qu'il demandoit Il criede toutes ses forces une seconde et une troisien fois. Enfin un matelot lui enfäit parvenir une; il la saisit entre ses dents, et cherche à regagner le rivage : un cri universelet desacclama- 
SUR L'île DE SYRA. CHap. VI. IO3 tions de joie se font entendre de tous les Syriotes; tous se flattoient de sanver jusqu'au dernier de tout l'équipage ; mais, à moitić chemin, on s'aperçut que la corde étoit trop courte, et cet accident les replongea dans la plus vive douleur. On crie au nageur de retourner au vaisseau, et de demander une autre corde. Cet homme intrépide, quoique exténué de lassitude, transi de froid, couvert à chaque instant par les vagues, sent renaître son courage, et reprend la route du vaisseau; mais enfin, forcé de céder à la fatigue et à la violence des flots, il est jeté sur les rochers, couvert de sang et de blessures. On tâche de le sauver clu naufrage, et de - l'arracher à la mort.

Les Syriotes ne furent point encore découragés; ils transporterent sur leurs épaules un bateau qu'ils mirent à flot, pour essayer d'aborder le vaisseau; mais il fut en $u$ instant fracassé contre les rochers. Une vingtaine de personnes se jetèrent dans une forte barque, et tàchoient, à force de rames, d'aborder le vaisseau du côté de la mer, pour tenter de sauver ceux qui étoient dedans : leurs efforts furent encore inutiles; ils manquèrent de périr, et eurent toutes les peines imaginables à re. 
gagner le port. Si le capitaine n'eût pas coupé la corde de la chaloupe, tont l'équipaye se seroit sauvé. Ce capitaine, témoin de tous les effortsdes Syriotes, et forcé d'en reconnoître l’inefficacité, prit le parti de se jeter à la mer; mais le reflux des vagues l'ayant entraîné sous la carêne, on ne le revit plus que le lendemain, la mer le jeta mort sur le rivage. Le désespoir des Syriotes de ne pouvoir porter des secours aux malheureux qui étoient dans le vaisseau, ne peut s'exprimer. La mort du capitaine et d'un autre qui l'avoit suivi, augmentoit encore leur douleur. On avoit vu périr trois personnes de celles qui étoient montées sur la quille du vaisseau, du froid excessif qu'il faisoit.

Ce désespoir iuspira une dernière tentative, qui réussit à arracher à la mort quatorze personnes de l'équipage. Quatre bons nageurs se lièrent à des cordes par le milieu du corps, et se tenoient tout près du rivage : aussitôt que la mer culevoit quelqu'un de dessus la quille, ou que quelques autres se jetoient à la mer, tous quatre s'y jetoient aussi, et si la mer ne les engloutissoit pas, ou ne les entraînoit pas sous le vaisseau, ils les saisissoient, et de terre, en tirant la corde, on les y ramenoit, en leus 
SUR L'íle de Syra. Chap. VI. io5 faisant vaincre l'effort des vagues. Quand les uns et les autres étoient sur le rivage, on ne pouvoit pas distinguer lequel étoit en plus grand danger, ni à qui porter plus promptement des secours. Ils étoient jetés contre des rochers, et converts de blessures : on les transportoit à mesure dans une caverne voisine, où un médecin du pays leur donnoit les secours convenables; il y avoit du feu, des alimens chauds, des habits et tout ce qui étoit nécessaire. Aussitôt que le naufragé étoit un peu revenu à lui, on le portoit sur les épaules dans une chambre où on avoit placé des matelas, et là, entre de bonnes couvertures, on achevoit, à force de'soins, de le rendre à la vie.

Pendant qu'on étoit occupé à soigner un matelot qu'on avoit retiré de l'eau, ainsi que les Syriotes qui l'avoient secouru, les vagues avoient entraîné de dessus la quille, un malhe urenx qui, du même coup, avoit été poussé jusqu’au rivage. Aucun des assistans ne s'en étoit ap)perçu. J'en étois plus près que personne et je fus frappé de roir remuer la jambe d'un lomme. Je me précipitai dans l'eau, je le saisis par la jambe, et les efforts que je fis pour l'en tirer me firent glisser. J'allois être entrainé 
avec lui, lorsque quelques Syriotes me saisirent par le milieu ducorps, et nous retirèrent ensemble. A peine fut-il sur le rivage, qu'on reconnut que c'étoit un pauvre 'l'urc, et je puis attester que j'ćprouvai dans ce moment une satisfaction égale à celle d’avoir sauvé les jours de mes parens les plusproches.

Quand on crut avoir sauvé tous ccux qui vivoient cncore, on entendit les cris d'un mousse de neuf à dix ans, qui demandoit du secours d'une roix si plaintive et si lamentable, que tous les cours en étoient déchirés. On ne pouvoit savoir où il étoit, ni d'où partoient ses cris. Emu jusqu'aux larmes, et onbliant mon insuffisance, jallois me jeter à la mer, sans réfléchir à l'inutilité de mon entreprise, et à la certitude du péril auquel je m'exposois. Je ne pus me soustraire à la douleur que cescris me faisoient éprouver, qu'en fuyant vers la ville.

Lę zèle des Syriotes, dans rette circonstance, ne s'est pas borné envers ceux qu'ils ont pu sauver, il s'est encore ćtendu sur les morts : à mesure que la mer les jetoit sur le rivage, on les portoit dans une chapelle près de la mer; ensuite l'évêque, à la tête de son clergé, descendoit en procession, suivi d'une grande foule de 
SUR L'île de Syra. Chap. VI.

peuple, et on les portoit à la Cathédrale pour leur donner une honorable sépulture. Par-tout sur leur passage, une duuleur vraie donnoit à leur mort des larmes sincères, Les femmes mê- loient leurs accens aux chants de l'Ég:iise, et chantoient, suivant l'usage des Grees, des hymnes lugubres etrimées, qui arrachoient des pleurs à tous les spectatemrs. J'en airetenu les paroles. Ah "pauvre père, panre mère! quand vous rece"vrez la funeste nouvelle de la mort de rotre « enfant! Ah! malheureuse épouse! où êtes-vous? "Vous êtes loin de croire que votre malheureux "époux soit dans ce cercueil ! Enfans, venez cm" brasser vos pères, arant quilils soient enfermés " dans un éternel tombeau! etc.

'Tous ces détails justifient pleinement les attestations que les Négucians de Niarseille et les Capitaines des vaissenx marchands, ont données de là charité et cle l'hospitalité des Syriotes (que nous rapporterons ci-après), e et que "parmi toutes les îles de l'Archipel, celle de "Syra a un droit particulier à la confiance et à "l'affection des François; sentimens que ces peu"ples méritent par l'attachement sincère quils " ont toujours témoigné à ceux de notre nation, " comme s"ils ne formoient avec nous qu'un seul "peuple." 
Lorsquion préscnta au capitaine Martichou ce certificat pour le signer (c'est un de ceux qui se sont acquis le plus de célébrité par leurs comses ), il le lut les larmes aux yeux, et dit: Oni, je le signerai de tout mon cœur, parce que c'est le plus exact de tous ceux qui ont été donnés.

\section{CERTIFICATS DES CAPITAINES,}

Concernant les services renatus par les habitans de l'ile de Syra, aux Navigateurs Franrois.

N o u s sonssignés, Capitaincs de vaisscans marchands, ponss's par les sentimens de recomuoissanee que nous ont inspirés les habitans de l'ile de Syra, par les bons et continucls services que nons en arons recus, déclarons et certifions à qui il appartiendra, que parmi les îles de l'Archipel que nons summes dans le eas de fréquenter ponr les affaires du commeree, celle de Syra a un droit partienlier à la confiance et à l'affection des Francois; sentimens que ces perples méritent par la confiance, le rèle particulier et l'attachement sincère qu'il ont toujours témoignés à cenx de notre nation, comme s'ils ne formoient avec nous qu'un seul peuple; s'empressant de nous donner tous les sccours qui ont été en leur pouroir, soit dans le 
naufrages que nous avons essuýés sur les côtes, soit dans $l_{\text {es attaques des corsaires euncuis, suit dins celles que }}$ nous arons sonffertes de la part des pirates qui infestoient les mers du Levant, lors de la dernière gnerre que les 'Tures ont sontenue, et dont plusicurs de nos vaissscanx eussent ité la proie, si ces insulaires n'avoient pas pris les armes pur nous défendre; soil encore dans les atteintes de la contagion, dont nos équipages enssent été la victime sans les sccours abondans qu'ils nuns unt donnés dans ces tristes conjonctures

C'est pourquoi nous déclarons volontiers, que tons les raisseanx Francuis trunveront dans cette île, des arantages qu'ils ne troureront nulle autre part dans l'Archipel, et. qu'ils peurent y mouiller avec une entière assurance. En

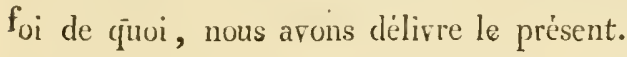

\section{A Marseille, le 2 Mars 178 I.}

Signés, P. A. Martichod, Rivière, J. J Je. Coste, P. Braxc, J. Couchier, B. Perou, Broutra, 11. Dounergue, Benussier, Reibaud, Antotne Catelin, Bone, A. Gutrard, H. F. Gumard, Darid, G. d'Allest, B. Murat, Boze, Coreif, Fournier.

Nous, Lazare de Gerin-Ricard, Conseiller du Roi, Lieutenant-Civil ct Criminel au siège 
de l'Amirauté de celte ville, en empêchoment, cerlifions a tous qu'il apparliendra, que les signalures ci-dessus apposies an bas du cerrificat qui les précède, sont récllement celles des personnes $y$ énonceies, lesqundles sont vérirablement Capilaines, ainsi qu'elles se qualifient, ayant toules signé en nolrc présence; en rémoin dé quoi mous arons signné. Donné à Marseille, le 16 Mars 1781.

$$
\text { Signé Gerin - Ricard. }
$$

Sccllè à Marscille, le 26 Mars r 78 r. Sigmé Tessier. Pour copic conforme à l'original gardé au burcan des Consulats.

\section{Signé Laneuville.}

CHRTI I I ATS des Négocians Francois, en faveur des mêmes habilans, sur le même siljet.

No o s soussigues, Négocians françois, quil arons residé dans diverses échelles du Levant, déclarons gue Jes habitans de l'ile de Syra ont totijours été portés à rendre de buns et fideles services à la nation francouse; quils se sont constamment prêtés, avec tout lé zéle ct la prédilection possibles, à domner, dans lem pays, toute sorte de 
SUR L'île DE SYRA. ChAP. VI. III secours aux bittimens françuis qui ont été dans la nécessite de reconrir ì lenis bons uffiees, soit dans les cas de nanfrage, d’accidens, de peste, attaques des pirates et ennemis de l’état, qui les unt poursuriris jusque dans le port mêmes de Syra; qu'en pareille circoustance, on a ru les habitans abandonner leurs fọers pour venir, à main arnée, secuurir et défendre, an piril de leur rie, les sujets dn roi, leurs biens, et l'honneur du pavillon francois, et les délirrer toujours des dangers auxquels ils étoient exposés. En foi de quoi, et à la requisition de ces fidèles amis, nous ne ponrons refuser ce témoignage à la rérité. Fait à Marseille, le ro Mars $173 \mathbf{r}$.

Signé, Hracivthe Remesat, Guys, P. Delisle, Lafleche, Hevry, Lasalie, Merte, Jacrees Arxtud, Haxcr, Jes. Truitler, Estio, Rocstain, J. B. Magr, Bourguignon, Louts RoLAND, JEAN-L,OUIS ROLAND, JESTINIEN G $\cap E \mathrm{~L} I N \mathrm{G}$.

Nous Echesin et Députés du Cummerce de cetle ville de Marseille, cerlifions et allestons que cence qui ont signés ci-dessus, sont lels qu'ils se qualifient; en foi de quoi nous avons signé ces présentes, et à icelles fait apposer le scean des armes do ladite ville 
de Marseille el commerce accoulumé, pour serviret valoir en cas de besoin.

Signé Hermite, Echevin.

T. Clary, Geor ge Audibert, Ferrari, L. J. Millot, P. Nicolas Testar, Bardon, Do L I E R l'aîné.

Pour copie conforme à l'original, gardé an bureau des Consulats.

Signé Laneu villa. 
SUR L'île de Syra. Chap. VII. Ii 3

\section{H A P I T R E VII.}

Du caractère et du génie des sjriotes.

$\mathrm{O}_{\mathrm{N}}$ ne peut réfléchir sur le caractère et sur le génie des peuples modernes de la Grèce, sans se représenter les beaux jours où cette heureuse contrée a produit tant de grands hommes dans tous les genres. II n'y a personne qui ne regarde les ancieus Grecs, comme une nation privilégiée de la nature, des arts et des grâces, et M. Gillies Anglois, dans ce qu'il a publié sur l'ancienne Grèce, n'a fait que répéter le témoignage qu'en ont rendu les peuples de tous les temps et de tous les pays. "C'est " de cette contrée, dit-il, qu'est venu tout ce " qui est en possession d'enchanter les hommes " depuis plus de vingt siècles accumulés; cette "brillante mythologie qui conserve des droits " indestructibles sur l'imagination, ces grandes " inventions poćtiques qui n'ont jamais pu être "égalées, l'idée du beau dans tous les arts,

Tome I. 
* les grâces, la pureté du guoût. Quand on parle " de modèles, on est obligé d'en revenir anx " anciens Grecs. Les Romains s'étuient lormés "sur eux; et dèsquon s'écurte de leurs trares, " on devient ridicule et on retombe dans la bar"barie.... Si on examine la cause à layuelle " on doit attribuer la précéminence que les Grees " eurent sur les autres nations, on trouvera que * les Grees eux-mêmes étoient dans la plus "forte persiasion que la contrée dans laquelle * ils vivoient, étoit spécialement favorable * à la dignnité de la nature humaine. Selon cux, " les voluptueux climats de l'Asie produisoient " desinventions ingénieuses, mais amollissoient " le tempérament des. hommes et les facon"noient trop aisément à la servitude; le ciel " rigonrenx des Européens, donnoit de la force: "et de l'agilité aux membres, et une fierté au"dacieuse à l'esprit, mais refruidissoit l'ima-, "gination, engourdissuit les délicates sensa"tions de l'ame: les habitans de l'orient et du " midi, étoient dégradés de la condition de "l'homme, par l'abus du pouvoir, tandis que.. "les fils tumbulens du nord et de loccident "étoient incipables, par leur ignorance et par * leur indocilité, de se soumettre à aucun sys- 
SUR L'île de Syra. Chap. VII. II 5

* tême régulier de gouvernement. Les Grecs * sents, placés dans une température favorable " au développement des idées, unissoient le con" rage à la capacité, tempéroient la sévérité " de leurs mours et la fermeté de leur carac" tère par la douceur des vertus aimables, et "jouissoient du double avantage de la liberté " et des lois. (1) «.... Voyez l'extrait dans le journal de Paris , 7 juillet 1788 .

(1) Voici à ce sujet deux passages d'Hippocrate et de Cicéron, rapportés par M. Paw : Recherches philosophiques sur les Grecs anciens, tom. I, sect. I, §. I0, pag. 90. "Hippocrate met en fait que les contrées où les " hivers sont extrêmement rigoureux, et les étés exces" sivement chauds, produisent une race d'hommes doués "d'une aptitude naturelle à cultiver les arts avec'suc"cès. De uere, aquis et locis. \$. dernier. Or, ne semble"roit-il pas, ajoute M. Paw, qu'Hippocrate ait voulu " par là désigner la Grèce, où le contraste des saisons "est plus frappant qu'en aucun autre point de la "terre, sous les mêmes latitudes?" Et dans la pag. 99, il dit aussi, "que Cicéron semble avoir pris la séche- ressse et la pureté de l'air, pour le principe createur "du génie subtil qui caractérisoit les habitans de "l'Attique." Mais d'après les principes que M. Paw a adoptés, et d'après ces suppositions sur la dégradation 
I16 PRECISHISTORIQUE

Il s'en faut de beaucoup que les voyageurs et les écrivains, soient également d'accord en-

tolale des Grecs modernes (voyez l'article suivant), il ajoute à ces autorités d'Hippocrate et de Cicéron, * que l'état actuel des choses démontre de la manière " la plus évidente, que des causes semblables ne sau"roient influcr sur l'esprit humain, si elles ne sont is "leur tour dirigćes par l'action des causes morales. - Dès que les philosophes et les savans disparoissent u d'un pays, comme ils ont disparu de la Grèce, il faut, - malgré le climat et malgré la nature même, que la * barbarie et la superstition enrahissent une terre sem- blable ; comme les ronces et les épines envahissent les "terres incultes. "Ces observations de M. Paw n'affoiblissent point les sentimens d'Hippocrate et de $\mathrm{Ci}$ réron. La question est de savoir si la Grèce, telle qu'elle est, produit des hommes doués d'une aptitude naturelle à cultiver les arts avec succès. Hippocrate et Cicéron, et tous ceux qui ont connu de près ces contrées, n'en doutent pas. M. Paw le nie dans une digrestion particulière qu'il fait sur la dégradation des Grecs modernes, que nous réfuterons ci-après. Cependant, pour développer ce que la nature nous a donné, il faut de l'application et de l'étude; sans cela, les talens naturels ne servent qu'à nous rendre plus vicieux. Le sentiment d'une personne d'esprit étoit, qu'elle n'avoit jamais vu un Grec médiocre; il faut qu’il exceile, soit en bien, soit en mal. 
SUR L'île de Syra. Chap. VII. II7 treeux surce que l'on doit penser des Grecs modernes. Les uns les peignent comme des hommes plongés dans lignorance et la barbarie, comme totalement abrutis, enfin comme une espèce tellement dégénérée, qu’ils ont perdu jusqu'aux dispositions physiques, sans lesquelles, toutes choses d'ailleurs égales, ils ne pourroient entreprendre de marcher sur les traces de leurs ancêtres; d'où l'on conclut qu'il n'y a rien de bon à attendre des Grecs actuels. Je crois pouvoir répondre à un jugement si tranchant et si injuste, que ces écrivains, ou n'ont jamais vécu avec ces peuples qu'ils traitent si défavorablement, et qu'ils ne les connoissent que sur les rapports de voyagneurs mal informés, ou qui voient mal les choses; ou bien que s'ils ont été à portée d'examiner ces peuples dans leur pays, ils ne l'ont fait que partiellement et superficiellement, et toujours d'après des préjugés reçus.

D'autres écrivains, meilleurs observateurs, ou travaillant d'après des mémoires plus sûrs, telsque M. Peyssonel, dans son dernier ouvrage contre M. de Volney; M. Guys, secrétaire du Roi, dans ses Voyages littéraires de la Grèce; M.l'abbéSestini, dans ses L.cttres sur les voyages 
au Levant, et M. le comte de ChoiseulGuuffier, dans ses voyayes pittoresques clu même pays; ces écrivains, dis-je, dont les deux premiers sont restés très long-temps dans le pays, l'un comme négociänt, l'autre en qualité de consul général de France, n'ont rien dit des vices des Grecs modernes, à qui on n'a à reprocher que ceux des autres nations; et noont insisté que sur ce qui ponvoit faire honneur anx peuples dont ils parloient. Ils conviennent à la vérité que les Grecs vivent dans une ignorance grossière; mais ils s'en_ prennent à l'état d'oppression et d'humiliation dans lequel ces peuples sont maintenus par les Tures, au défaut absolu de tont moyen de s’instruire, enfin à l'esprit même du gouvernement sous lequel ils vivent; maisces auteurs ajoutent qu'au milieu même de ces ténèbres de l'ignorance, ils ont encore recomu des traces de cette fúcondité d'esprit, de ce génie qui a caractérisé cette nation, et qui lui a assuré pour tonjours une supériorité décidée sur tontes les autres. "Le germe du génie ct des " alts, dit M. de Pejssonel, ihid. pag. 295, n’est "point entièrement étouflé chez les Grecs : il "n'est pas nécessaive de leur rendre les riches as scines de la nature; ils les ont sous les yeux 
SUR L'îlE DE SYRA. CHAP. VII. II "dans le plus beau pays, el sous le plus beau " climat de la terre, où la nature se montre " dans toute sa pompe et tout son éclat. Il * n’est pas nécessaire de leur rendre les riches " scènes de l'état sucial; ils en sont tous les jours " témoins dans une des plus nombreuses sociétés "qui existent. Il ne faut que leur rendre les "modèles dars tuns les genres, que leurs an" cêtres ont fournis aux siècles passés, jrésens "et à venir; et bientôt ils les imiteront."

Si après de telle's autorités, il métoit encore permis de donuer mon opiuion sur ce qui concerne ma nation, ( car ma famille, quoique originaire d'Italie, étant établic en Grèce depuis plus de deux sičcles, doit être réputée grecque;) si, dis-je, mes assertions pouvoient n'être pas suspectes, il me seroit aisé d'avancer et cle prouver par des faits, qu'il n'y a pas de nation qui, réduite au même état d’humiliation, d'avilissement et de détresse, ne fût incomparablement plus abrutic. Opprimés impitoyablement par les Tures qui sont devenus letrs mât res depuis leur conquête, ils s'estimeroient encore heureux, si les autres Europécns ne se joignoient sonvent à eux; et si en enctérissant sur les opprobres quils leur font soulinir, ils n'a- 
chevoient de rendre le joug tout-à-fait insupportable. S'élève-t-il, par exemple, un différend entre un Grec et un Européen? celui-ci parvient presque toujours, à force d'argent ou de crédit, quelques torts qu’il ait, à écraser son adversaire, et souvent à le faire condamuer à la prison ou au moins à la bastonnade. Je le demande; quel peuple pourroit soutenir une pareille épreuve, et ne tomberoit bientôt dans un état d'engourdissement et de barbarie, pire que celui où se trouvent les Grecs, et tel que celui où quelques auteurs voudroient persuader quils sont en effet tombés?

Mais venons aux Syriotes : placés à-peupries au centre des Cyclades et au milieu des deux Grèces, ils doivent, sans contredit, participer aux prérogatives morales des Grees, d'autant plus qu'on sáit que l'on trouve communément chez les insulaires, je ne sais quoi de plus libre et de plus saillant dans le génie, que chez les habitans du continent; et en effet, parmi les grands hommes dont les talens ont illustré la Grèce, on remarque que, proporlion gardée, il en est plus sorti des îles que de la terre ferme. Homère étoit de Chio, suivast la plus commune opinion; Salanine, 
SUR L'île de Syra. Chap. VII. I2t Rhodes et plusieurs autres îles, ont revendiqué I'honneur de lui avoir donné le jour: l'historien Théopompe étoit aussi de Chio; Euripide étoit né à Salamine; Alcée, 'Terpandre, Pittacus, Sapho et Théophraste, à Lesbos; Théocrite, selon quelques-ıns, Apèle et Hippocrate à Cos; Pythagore et l'astronome Aristarque, à Samos; Phérécyde, à Syros; Simonide le jeune, à Céos, aujourd'hui Zéa; enfin plusieurs autres qu'il seroit trop long de nommer, et qui sont nés dans d'autres îles.

Comme les Syriotes se sont toujours tenus renfermés dans leur île, occupés à la culture de leurs terres, et qu'ils n'ont commencé que depuis peu à communiquer avec leurs voisins et avec les étrangers, ils ont encore eu moins d'occasion que les autres insulaires de dérelopper leur génie et de se faire connoître; aussi passoient-ils dans l'Archipel, pour des gens extrêmement simples. Mais depuis qu’ils s’artonnent à la navigation, et qu'ils vont faire le commerce, soit à Constantinople, soit à Smirne, soit à Salonique, ou dans d'autres ports de la Turquie, ils ont guéri les esprits des préjugés que l'on avoit pris sur leur compte : leur génie s'est développé, et ils ont prouvé qu'un 
Syriote vaut au moins le pius spirituel et le plus éclaniré des habitans des autres îles; et en sénéral on ne sanroit leur refinser plus de vivacicé, avantare qui résulte sans doute des instructions assidnes de leurs pasteurs en matière de religion; car on peut assurer que, eu égard au nombre des Syriotes, il n'y a peut-ètre pas de peuple, même dans torate la chrétienté, qui reçoive plus de secours spirituels, et 'Tuurnefort lui-même atteste que leur clergé est le plus instruit de tont l'Archipel.

Vifis, pénétrans, mes compatriotes ont toujomıs montré les dispositions les pius henremses pour les arts, et ils ont réussi dans tous. Ils sont renommés dans le Levant par leur gnoût pour la danse et la misique; le luth, et la lvre, esijece de violon à trois curdes, sont les instrumens sur tout, qu'ils connoissent le mienx. Quelques ex(mp)!es vont prouver quils sont capables de réussir également dans les sciences. Il n'y a pas long-temps que quelquiun apporta à Sira une de ces horloges de bois q ion tait en Allemagne: plusieurs jeunes-gens de l’ile se mirent à la contrefaire et réussicent si bien, quils en vendirent jusqu'à \& ou 5 écus la préce. J'ai comnu un tailleur qui a dessiné à la plume une 
SURL'îlE DE SyRA. ChAP. VII. I 23 frégate Françoise qui mouilloit dans le port. Le Capitaine, à qui il offrit son dessin fut si content de l'exécution, qu'il lui fit un présent honnête, et l'assura qu'il conserveroit avec sand soin cette production, pour faire voir en France ce qu'un Grec, qui n’a pas reçu les premiers élémens du dessin, est capable de faire. De mon temps encore, un jeune Ecclésiastique, Chancelier de l'Évêque, apprit seul à peindre à l'huile, et fit plusieurs tableaux que l'on roit dans les Églises de Syra et dans d'autres parties de l'Archipel. On conçoit bien que ces tableaux ne sont pas du dernier fini, mais ils 'ne laissent pas de faire honneur à leur auteur. Je pourrois citer encore bien des Syriotes qui ont appris des métiers sans maîtres.

Quant à la sagacité dans les afaires, les habitans de Syra ne le cèlent à aucuin de ceux qui vivent comme eux sous le ciel de la Grèce. J'ai vu un homme de la campagne qui avoit demeuré quelque temps à Constantinople: personne ne discutoit une afrare mieux que lui; il avoit la tête la mieux organisée, l'esprit le plus juste, la logique la plus saine; cet homme, d'argument en argument, pressoit son adversaire avec tant de viğueur, qu'il le réduisoit bientôt 
au silence. Dans le temps que la flotte Russe croisoit dans l'Archipel, il s'ćleva entre le peuple de Syra et ses Primats, une dispute concernant quelques affaires d'intérêt de la communauté. Plusieurs Officiers Suédois, Danois et Livoniens au service de la Russie, que l'on avoit mis à terre dans l'île pour y rétablir leur santé, témoins des intrigues multipliées, des cabales, des attaques, des répliques, enfin des moyens pressans employés de part et d'autre pour faire réussir une cause, avouèrent avec surprise que, dans aucun pays de l'Europe où ils avoient voyagé, ils n’avoient trouvé autant d'énergie et de chaleur à soutenir des prétentions, ni la même subtilité pour se procurer des raisons victorieuses ou au moins favorables. J'ajouterai encore un trait qui, s'il ne prouve pas la solidité du jugement de l'un des acteurs, est au moins un échantillon de l'adresse des Syriotes à faire valoir leurs causes bonnes ou mauvaises. Deux paysans disputoient sur l'âge de la lune; chacun d'eux, à l'aide des épactes et de quelques autres calculs ( car on n'a pas même dans l'île la commodité des almanachs ); chacun d'eux, dis-je, appuyoit son opinion des raisons qu'il croyoit les meilleures; quand celui qui soutenoit que la 
SUR L'île de Syra. Chap. VII. I 25

lune étoit déja nouvelle, levant les yeux, l'aperçut au ciel : Tiens, dit-il en triomphant à son adversaire, la soila nonvelle. L'autre regarde: fié bien, dit-il, elle ne doit pas l'être; et, sans se déconcerter, il continuoit à piouver par ses raisomnemens que la lume ne devoit commencer qu'à telle époque: l'ardeur de la dispute qui l'emportoit étoit telle, que s'en rapportant plus à son calcul quaux faits, il croyoit pouvoir mettre la nature en défaut. Que faut-il conclure de tout cela? que les Grecs modernes sont encore les mêmes, quant aux dispositions physiques pour les sciences et pour lés arts; que ce que certains anteurs ont avancé sur leur incapacité et sur leur abrutissement, est absolument faux; que si le gouvernement sous lequel ils vivent, moins oppresseur à leur égard, leur permettoit de remettre en rigueur dans leur pays, la culture des arts et des sciences dont le goût est né avec eux, s'il entreprenoit d'y exciter l'émulation si nécessaire pour leurs progrès, on verroit sans doute naître, en peu de temps, une foule de génies créateurs, qui ne le céderoient en rien à ceux dont la Grèce a été autrefois si féconde. On verroit les enfans de çes mêmes Grecs, qui seuls et 
sans guide, ont osé, par la seule impulsion de leur génie, s'élever d'un vol rapide au dessus de l'atmosphère ténébreux de l'ignorance, daıs lequel le reste de la terre demcuroit plongé, laisser bien loin au-dessous d'eux, ceux qui ont entrepris de les suivre; on verroit, dis-je, dans un siècle éclairé comme le nôtre, où les moyens sont si multipliés, et les connoissances si aisées à acquérir, leurs cnfans inventer, perfectionner, et mériter par la sublimité de leurs productions, les applaudissemens et l'admiration des autres peuples.

Quoi! me dira-t-on, vous regardez donc les Grecs comme supérieurs aux autres hommes, comme des hommes surnaturels? Non ; je sais que presque tous les pays ont fourni de grands sujets, des génies rares et sublimes; mais personue ne contestera que la Grèce n'en ait produit plus qu'aucun autre pays, et pour juger par analogie, et par la connoissance que j'aide de cette contrée, je suis persuadé qu'elle n'a rien perdncie sa fécondité, et qu'elie n'attend que des circonstances favorables pour la dévelopjer. J'ai tonjours entendu dire à Rome qu'on avoit observé que de 100 jeunes Levantins, à peine 
en trouvoit-on 2001130 sans aptitude, et yu'au contraire sur roo Itatiens, à peine y en aroit-il 20 on 30 qui annoncassent des talens; et je me rappelle qu'un Profésseur de Théologie qui venuit donner des lecouns daus notre collégée, où it n'y avoit gureres que des Levantins, nous disuit: Mes enfans, vous ne sanries imnginer quelle salisfaction je tronve a vons domner des lecons, mais avec quelle peine je vais dans nos écoles, el combien je'sonffre de me voir obligé de rëpeiter si sonwent a mes écoliers les réponses aux argumens que je leur propose; iandis quavec vons au contraire, je suis quelqueJois contraint d'être sur mesgardes en argumentant.

Je ne nierai pas pour cela que les Italiens ne l'empoitent sur les (jrecs par la finesse du jugernest. Ce seroit démentir uné opinion que M. Rollin établit daus le parallèle quil fait de Démusthène el de Cicéron, d'Humère et de Virgrile.

Homère et Démosthene mettent dans leurs ouvrages plus de f'u et plus d'invention; Virgile et Cicéron, plus d'ordre et plus d'ayrément, ce qui est l'effet de la délicatesse du jugrement. 
128 PRÉCISHISTORIQUE

Rollin le prouve encore par l'esprit du gouvernement d'Athènes, et de celui de Rome. La politique de celui-ci est infiniment sujpérjeure à celle des républiques grecques. On voit, par exemple, Athenes aliéner contre elle l'esprit de 800 familles, en privant du titre de citoyen ceux qui n’étoient pảs nés de père et de mère Athéniens. Rome au contraire, les multiplia tant qu'elle pût, et par là, les soutiens de la république, puisqu'elle accorda aux habitans de plusieurs villes, le titre et les droits de citoyens romains.

Entrons encore dans quelques détails particuliers sur les Syriotes: extrêmement hospitaliers, sur-tout envers les François, ils sont inexorables sur l'article du vol. On s'y rappelle d'un jeune homme, qui avoit volé des choux, et dont toute sa famille sollicita la punition : il fut comdamné à l'exil. Cette qualité des Syriotes est si connue, que clans les maisons des négocians françois ou autres, on les préfere toujours, pour servir, à ceux des autres pays.

Ils sont agiles, robustes et très-courageux : dans tous les temps ils ont montré de la bravoure. Dans l'île déserte de Jura, qui en est une dépendance, un certain George Xanthahi, renommé 
SUR L'île DE SyrA. Chap. VII. I29 renommé dans tout l'Archipel pour son couragge, arecun autre compagonon, surprit et déSarma dix-huit Zantiotes. Ce même George à donné plusienrs traits de valeur contre des brigands, pendant la guerre, et le séjour des Russes dans les îles de l'Archipel. Nous en avons rapporté quelques faits dans le chapitre précédent. Un des neveux de Xanthahi, nommé Antonio Rossi, s'opposa seul au fameux Mitromara, sans pezr, qui, Avec trente deux Albanois, avoit fait une descente à Syra, pour la ravager; ce jeune homme, car il n'avoit pas plus de vingt à vingtdeux ans, s'étant emparé d'un poste par lequel la troupe des brigands devoit passer, se coucha par terre, et appuyant son fusil sur la pointe de son pied, à la manière des Albanois, il ajusta si bien, que Mitromara fut blessé aux épaules, avec deux autres des siens, et qu'il fut obliģé de se rembarquer.Les Syriotes, pendant ce même séjour des Russes dans la mer blanche, s'étoient tellement acquis de la réputation dansplusieurs rencontres qu'ils eurent avec beaucoup deces brigands, qu'ils ne les craignoient plus, quelque fût le nombre de leurs ennemis. Ceux-cian contraire en conçurent une telle frayeur, qu'ils n’osoient plus descendre à Syra.

Tome $I$. 
Par beaucoup dautres exemples que nous pourrions encore citer, on doit en conclure, que si les Syriotes étoient pliés à la discipline militaire, ils formeroient de très-bonnes troupes, et il s'élèveroit parmi eux d'excellens Officiers. Si un fait particulier pouvoit décider de l'intel ligence de tout un peuple, on pourroit dire que les Syriotes réussiroient parfaitement dans la discipline militaire; on sait que les Grecs ont été fámeux dans l'art de la guerre. Un Syriote, de la famille Stephano, surnommé Cagnazzo, étant passé au service de la maison d'Autriche, s'y étoit comporté avec tant d'intelligence et de bravoure, que Marie Thérèse l'honora de la plus grande protection, et l'éleva à des places distinguées parmi ses officiers. Cette princesse l'ayant mis d'abord au rang des nobles, il auroit pu prétendre par ses talens militaires, aux premiers sangrss de l'armée, s’il n’eût été tué à la prise de Prague; monté le premier à labrêche, il y arbora son drapeau : cette action héroïque ayant ajouté à la jalonsie quion lui portoit déja il reçnt par derrière un coup de feu dont il expira sur le champ. Sa qualité d'étranger n'avoit pas peu contribué à lui susciter un grand nombre d'ennemis. L'Impératrice avoit fait venir aussi un 
SUR L'îlE DE SYRA. CHAP. VII. I3I de ses frères nounmé David, qu'elle fit mettre au collège. Ce jenne Syriote $y$ fit de yrands progrès; mais s'étant appliqué à l'étude avec trop d'ardeur, il en perdit la lête : on fut obligré de le renvoyer à Syra, où je l’aj connu. Il étoit un grand nageur, et l'on raconte qu'il avoit passé la mer à la nage, de Tine à Syra, ce qui fait un trajet d'environ dix-huit milles.

Les Syriotes sont doués aussi d'une grande subtilité dans les organes optiques. Dans un temps serein ils voyent de Syra, les paysans marcher et labourer sur les montannes de Tine, c'est-à-dire, à la distance de plus de six lieues Ils voient aussi très-aisément les cailles qui se tiennent cachées, et qui reposent sous l'ombre despetites broussailles de thym, sauge, et autres plantes; c'est alors qu'ils les couvrent aussitôt d'un filet, et quils attrapent ainsi quantité de ces petits oiseaux.

"Onsait, dit M. Paw, que la grrande perfection des orgranes optiques ćtoit un caractère national qui distinguoit les Grecs de tous les autres peuples; et quoique nous connoissions aujourdhui les différentes races d'hommes ré. pandues stu cette planete, depuis le pays des Esquimaux jusquà la terre de Feu, et depuis les 
côtes d'Afrique jusqu'aux îles de la mer di sud, on n'a pas découvert, parmi tant de variétés et tant d'espèces, une seule nation en qui le globe de l'œil fut si considérable, et l'orbite si évasée, qu'clle étoit souvent dans les Grecs. Ce n'est pas sans raison, dit M. Winchelman, qu'ils attachèrent, dés le temps d'Homère, à cette faveur de la nature, le caractère de la plus sublime beauté, car une grande lumière est sans comparaison plus agréable quine foible lueur."

Or, tout le monde sait qu'une garande quantité d'individus des contrées de la Grèce moderne, sont doués d'une orbite bien évasée, et d'un globe consiclérable.

"Quoi qu’il en soit (c’est toujours M. Paw qui parle) de l'influencedes causes particulières, aucun seus n'étoit plus perfectionné dans les Grecs en général, que celui de la vue; c'est à cet avantage qu'on doit en partie attribuer les étonnans progrès qu'ils firent dans tous les arts qui dépendent du dessin, tandis que les Egyptiens, qui avoient les yeux foibles, n’y purent même atteindre à la médiocrité, et couroient sans cesse dans une carrière où ils n'avançoient point : il. leur étoit impossible de saisir exactement le contour d'un corps animé, et les belles formes 
SUR t'île de SYra. CHA P. VII. \33 s'échappoient, pour ainsi dire; dè dessous leur crayon. "

Cette citation de M. Pav' doit servir à confirmer ce que j'ai dit lans, ce chapitre, de la conformité du caractère et d’u jónie des Grecs modernes, avec ceux des anciens auss. à lui répondre dans le chapitre suivant, au sujet de la dégradation des Grees modeines:

Nos insulaires apprennent airssi trés-facilement, les langues, et les parlent assez bien; plusienrs d'entre eux, même parmi les gens de la campagne, en parlent quatre, savoir: Ia Grecque, la Turque, l'Italienne et la Françoise. Je dois rap porter à ce sujet un évènement que je tiens de la personne même à qui il est arrivé. Un nommé Jean Rousseau travailloit à la terre, au couchant de lîle, dans un quartier qu'on appelle Ghalissia, où il y a un port qui, quoiqu’à l'abri des vents du nord, est cependant très-dangerenx par les tourbillons qui sy renferment. Il apperçoit une frégate du Roi yni y aborde; Rousseau, effiayé du danger aupuel elle est exposéc, abandonne son travail, et court de rocher en rocher, en criant au capitaine. Celui-ci lui envoie la chaloupe, qui amène aussitôt le paysan à son bord, lequel lui décourre le danger dont il 
I34 PRÉCIS HISTORIQUE est menacé; il s'offre en même temps de luxi servir de pilote, et de le conduire vers un antre endroit, au midi de l'île, où il pourra jeter l'ancre en sureté. Le Capitaine suit son avis, admirant l'attachement de cet homme à la Nation Françoise, ainsi que la facilité avec la quelle il en parloit la langue, qu'il avoit apprise au service de M. le Marquis de Castellane Ambassadeur du Roi à la Porte. 
SUR L'I L E DE SYRA. CHA P. VIII, I35

\section{H A P I T R E VIII.}

Contrle réfutalion de M. PaW, sur ce qu'il dil des Grees modernes.

J' A ro o s déja terminé l'article précédent sur le caractè e et le z̧énie des Syriotes, lorsque j’ai vu paroitre les Recher ches philosophiques de M. Paw , sur les Grecs. Sans vouluir entreprendre ici la critique de cet ouvrage, je suis forcé d'aroner que la lecture du chapitre intitulé; dégradation des Grecs modernes, m'a infiniment touché. L'auteur attaque principalement ce que Munsieur le Comte de Choiseul-Gouffier . dans son excellent ouvrage sur la Grèce pittoresque, et M. Guys, dans ses Voyages littéraires, ont dit d'avantagreux et de raisonnable de l'esprit et du grénie des Grecs modernes. Il va même plus loin; il prétend que ces peuples sont tombés dans un tel degré d'abrutissement, que leur moral et leur physique forment un 
contraste, frappant avec ce que les historiens nous ont appris de ceux de leurs ancêtres.

D'après les asscrtions paradoxales que plusieurs écrivains judicieux ont déja reprochées à cet anteur dans ses ouvrages sur les Chinois, les lígyptiens, les Suisses et les Américains, j'aurois dû peut-être me dispenser de relever cellesqui regardent les Grees molernes; mais sachant que le plus grand nombre des lecteurs craint de se livrer à un examen sérieux, qu’il se laisse éblour par des réflexions hazardées et des raisons superficielles, j’ai cru qu'une courte digression sur cet ouvrage, ajoutée au précis historique de l'île de Syra ne seroit pas inutile.

"On peut compter, dit M. Paw, parmi les " livres les plus utiles qui aient jamais été "écrits, le prétendu voyage littéraire de la * Grèce, par M. Guys, de l'Académie de Mar"seille. :

Après un jugement si décisif, on croiroit que M. Paw va se donner la peine de se motiver, mais il n'en fait rien : il ne croit pas devoir plus de ménagement à un écrivain qui a reudu justice aux Grees modernes avec beaucoup d'impartialité et de discernement.

"Il veut y démontrer, continue M. Paw, 
Sur L'île de Syra. Chap. Tíll.

« que les Grecs sont cneore aujourdhui tout ce "quils liurent dans lautiquité. "

Jamais M. Guys, jamais M. de Choiseul, nont avancé que les Grecs modernes fussent aussi exercés dans les arts, aussi profonds dans les sciences, que les anciens; mais ils ont dit, et cela est vrai, que la nature leur a donné toutes les dispositions morales et plysiques, pour devenir ce que leurs ancêtres ontété. Vöici leurs opinions, celle de M. Peyssonel, celle de tous ceux qui ont connu cette nation, qui l'ont vue de près, et qui abandonnent les systêmes, les préjugés et les paradoxes aux philosophes du jour, pour s'en tenir à la simple ct exacte rérité.

Pour prowver ce cu'il avance, ivi. Guys rapporte plusicurs excmples :j’en ai déja cité moimême quelques-uns; mais s’ils ne suffisent pas à M. Paw, qu’il s’informe si les jeunes Grees qui sont à Rome, n'excellent pas dans les sciences; qüil interroge les winiversités de Padone et des autres villes de l'Italie, il verra s'ils niy font pas des progrès aussi rapides que surprenans. "Telle cst la chimère de cet hommc-là, " (ccte manière de s'exprimer passera diffici"lement pour être polie), (qui aplès avoir négo- 
* cié à Constantinople, s'est cru en état de juger a les nations, sans mêne appeler à son secours " les lumières de la philosophic."

Il semble d'abord que M. Paw prenne les négocians des nations commerçantes pour des colporteurs d'Allemagne; ignore-t-il qu'en Angleterre, en Hollande, cn France, et chez d'autres peuples maritimes, l'éducation despersonnes que l'on destine au commerce, est très-soignéc? que le fameux Fahner, qui exercoit avec tant d'éclat l'emploi d'Ambassadeur d'Angleterre à la Porte, et à qui Voltairc a dédié un cle ses ouvrages; que mille autres qui ont brillé dans des postes aussi importans, ont commencé par faire le commerce? étoit-il done défenduà M. Guys, pendant le très-long séjour qu'il a fát à Constantinople, d'étudier en homme éclairé, et non en philosophe aveugle, les Grees sous tous les rapports? Ce n'est qu'après les avoir vîs, les avoir connus, qu'ils s'est cru autorisé à les juger. On pourroit demander à M. Paw, s'il se conduit ainsi, lui qui décide, qui tranche, et qui condamne une nation sans avoir voyagé chez elle, que de son cabinet.

«On ne sauroit, poursuit M. Paw, trouver des expressions pour dépeindre l'état d'avilis- 
SUR L'Íle de Syra. Chá. VIII. 139 "sement, où les Grees modernes sont tombés "et où ils sont tombés, par leur propre faute; "car les Tures qui leur ont laissé tous leurs "monatères, n'anruient jamais pensé à leur "óter les écoles, s'il en avoient vouluavoir.C'est - le fanatisme qui a été la source de tous leurs "maux, et ce sont enx qui ont resserré de "leurs mains les nouds du bandean qui les "avemigle:"»

- M Paw, fidèle à son systême, écarte de lui touf ce qui pourroit le faire cronler : il fait semblant de ne pas savoir que la maniere dont les 'Iucs' ont fait leur conquête, est bien différente de celle des antres peuples conquérans de l'Europe. Les Francs asservirent les Gauluis; mais ees deux peuples bientồt réunis par lá ReliGion et par des alliances, ne formèrent plus qune nation, et torres les idstinctions entre los rainqueirs et les vantris disparment. Les 'Tucs au contraire', après 'avoir soumis lés Gı ecs, leur laissient, à la vérité, lemr religion; mais ils ne formerent avec enx aucune alliance; ils rejetèrent toute union, ils établirent enfin un mur de séparation si impolitique entre le peuple conyuérant et le peuple conquis, que 
celui-ci fut regardé comme proscrit et qu'il gémit encore dans l'esclavage et l'avilissement.

Elle n'existe que trop sans donte, cette cruclle dépendance, cette humiliation profonde de la nation Grecque! quel peuple accablé d'un despotisme rigoureux, qui pèse sur lui depuis près de 400 ans, ne seroit pas encore plus avili? Mạis comment ne pas admirer l'éncruaic de ce caractère, qui les a soutenus pendant des siècles d'oppression? Plaignons-les de n'être pas libres, c'est le dernier terme du malheur mais ne désespérons pas de ce qu'ils peuvent devenir un jour dans la: carrière des, arts et des sciences.

Avant d'être injustes envers eux, et de leur reprocher d'avoir dégénéré; il faudroit,réflérrs chir sur leur situation, se bien pénétrer de la constitution, de l'esprit et du gouvernement 'Ture, et voir si les. Grecs sont les maîtres, de s'adonner à la perfection des arts et à l'étude! des sciences.

Ce n'est point le fanatisme qui perpétue leurs maux; ce n'est point lui qui les tient dans cette nuit, épaisse, où, on les suppose entièrement plongés. Par-tout les gouvernemens font les 
SUR L'île DE SYra. ChaP. VIII. I 41 hommes. Dans quelles horreurs les guerres religieuses n’ont - elles pas précipité la France, l'Angleterre, l'Allemagne, l'Europe entière ? Aujourd'hui que leurs traces sont effacées, ne voit-on pas.les sciences et les arts fleurir chez les nations éclairées? mais à qui est-elle due cette grande et heureuse révolution? aux états qui ont accueilli les grands hommes clans tous les genres; aux Princes qui les ont appelés ou encourağés; à François premier, à Come de Médicis, à Econ X, à Lonis XIV, à Pierre-leGrand, à Frédéric II , à Cathérine II ; chez les Grecs au contraire, le despotisme, ce monstre né de l'ignorance et de la Barbarie, s'est appésanti su eux; il a étouffé leurs dispositions, arrêté leurs progrès, enchaîné le génie dont ils ont hérité de leurs ancêtres; en rédoutant leur instruction, il l'a gênée de tant d'entraves, qu'elle ne sauroit franchir le terme qu'il lui a assigné.

"En lisant des légendes, dit M. Paw, et en " disputant sur dẹs chimères, ils ont oublié les "ćlèmens des sciences et des métiers. "

C'est aux lecteurs des légendes, et à ceux qui disputoient sur les cathégories d'Aristote, que nous devons en Europe, la conservation des 


\section{P R E C IS HISTOR I Q U E}

sciences; ce n'est que par eux que plusieurs connoissances économiques et des manufactures se sont établies ou conservées en Europe. Sur cet article qui a été traité avec tant de légéreté par nos philosophes, je renvoie M. Paw, à J'histoire de Danina, sur les révolutions de l'Italie.

C'est vers la fin de leur Empire, lorsque leurs controverses avec l'Ëglise Romaine se furent élevées à leur comble, que les Grecs furent le plus agités de cet esprit de dispute; c'est même alors que très-instruits pour le temps, pendant que toutes les nations étoient presque plongées dans l'ignorance, ils se retirerent en Italie et en France, et qu'ils y apportèrent le germe des sciences et des arts.

Si M. Paw se donnoit la peine de lire l'unvrage de Flachat, sur le génie et l'industrie des Orientaux, il y verroit une longue énumćration des machines ingénieuses qu'ils ont iuventées, et il ne pourroit refuser son atmiration aux habitans de l'île de Chio.

Quant ì ce quil ajoute, "que ce peuple est "rentré en enfance; qu'il est dévenu le plus vil " Gardeau de la terre, et l'opprobre de ses areux "dont il foule aux pieds les tombenux, saus «même les connoître, " nous lui demanderuns 
SUR L'íle de Syra. Char. VIII, I $4^{3}$ si les lumières de la philosophie, qu'il ne cesse de réclamer, l'ont bien servi dans cette dure inculpation, que nous n'aurons pas d'ailleurs beaucoup de peine à réfuter.

A Smyrne, à Salonique, à Scalla-Nova, à la Morée, à Candie, à Volo, dans toutes les échelles du Levant, une quantité immense de bâtimens de différens ports de l'Europe, font des chargemens en tout genre; des blés, des huiles, des vins, des soies, des lames, du coton, du miel, de la cire et autres denrées de première nécessité, se transportent de la mer Blanche jusque dans la Baltique; elles sont le fruit des travaux et de l'industrie des Grècs, qui en font jouir tous les peuples de l'Europe, et les Grecs sont encore, aux yeux de M. Pasv, le fardeau de la terre.

"M. Guys, dit-il, ose mettre en fait que les "Athéniens modernes, plongés dans une nuit * aussi profonde, ont l'oreille si subtile, qu’ils " ont retenu par tradition la bonne prononcia"tion de la langue grecque; ce qui supposeroit, "selon lui, que leurs organes ont aussi con"servé leur flexibilité, par l'influence du cli" mat; mais pour démontrer la fausseté abso"lue cl'une assertion si absurde et si ridicule, 
144 PrécIS H ISTORIQ U E

"il suffit d'observer que de 70 jargous quon " parle maintenant dans la Grèce, celui des "Athéniens fut, dès l’an 158 r , jugé le pluscor"rompu et le plus horrible de tous, de l'aveu " mêne des Grecs; ce qui n’est pas surprenant, "quand on sait que déja vers l'an ı 30o, on par"Joit vulgairement à Athènes un dialecte fran"çois, qui se répandit jusqu'en Asie après la * prise de Constantinople par les Francs, ainsi "que le rapporte Ducange, etc. "

On se jetteroit dans de trop longues discussions, si l'on vouloit approfondir la question de l'influence du climat sur la flexibilité des orgatnes; mais sans connoître les 7o jargons qui se parlent maintenant dans la Grèce, s’il faut en croire M. Paw, nous pouvons arancer qu'un Gree, de quelque contrée qu'il soit, est parfaitement entendu, et se fait pareillement très-bien entendre de tous ses compatriotes. Dans aucune des langues modemes, on ne trouveroit peutêtre cet avantağe. An surplus, il ne seroit pas étonnant qu'aux époques citées par. M. Pav, les Athéniens, soumis aux Européens, cussent parlé un matuvais gुrec, puisqu'une quantité de filmilles Françoises et Italiennes s'étoient établies dans ces contrées. Il paroît, par le témoignaģe de 
SUR L'Ất, E DE Syra. Chap. VIII. I 45 de plusieurs voyagenrs, qu'on parle maintenant d Athenes un assez bon grec.

M. Paw traite, arcec la même légéreté, le royage pitturesque de $\mathrm{M}$. de Chviseul-Gouffier, qui, après aroir parcouru la (iréce en philosophe éclairé, ne craint pas d'avancer clans son discuurs préliminaire, que si les Grecs étoient libres, et pouvoient s'appliquer aux sciences et aux arts, ils deviendroient ce qu'ils étoient au temps de Périclès.

A cette judicieuse observation M. Pav répond, que des hommes de cette nation lui ont avoné , que s'ils parvenoient à l'indépendance, le premier usagre qu’ils feroient de la liberıé politique, consisteroit à entreprendre une graade guerre de religion, où les prétendus Urthodones et les prétendus Schismatiques s'égorgeroient jusquau deraier sompir, puur des mots qu'ils ne sarent mệne pas prononcer comme il faut. "Il n'y a pas parmi nuus (ont-ils dit encore à M. Pdw), un homme tant suit peu religicux, qui ne sc laissât plutôt mettre à mort, que d'apprendire un art tel que celui de statuaire, qui est, selon la décision des Caloyers du mont Pentelique, le ciernier degré de l'idolatrie. «

M. Paw nous permettra de lui observer que iome $l$. 
des oui-dires ne sont pas des vérités historiques; quil est rare qu'un homme raisonnable, qu'un vrai philosophe les regarde comme des autorités à l'abri de toute atteinte, et que ce n'est pas sur des témoigrnages incertains, qu'il faut condamner une nation entièe, quand l'histoire, sur-tout de tous les temps et de tons les lieux, les contredit, si victorieusement.

Les peuples les plus ignorans et les plus barbares, les Francs, les Guths, les Germains, les Saxons, nont-ils pas clonné naissance anx Francois, aux Allemands, aux Anglois, etc. A quelle distance ceux-ci n’ont-ils pas laissé lcurs ģrossicrs ancêtres? et les descendans de ces Grecs qui ont farit l'admiration de la terre entiere, eux qui tirent leur orighine d'une espèce d'hommes réunissant, au plus haut degré de perfection, tout ce que la philosophie, la poésie, l"éloquence et les beaux-arts ont de plus subline et de plus exquis, les descendans des créatems de tant de prodiges, auroicnt sculs le malheur d'être frappés à jamais d'une stupidité invincible! et si, dans des siècies de barbarie (ainsi que nous l'avons remarqué à l'article précédent), si, dans la nuit la p'us profonde, les anciens Grecsen oni dissipéles prestigesavec tant d'éclat, 
SUR L'île DE SyrA. CHAP. VIII. I 47 ne sera-t-il pas permis aux Grecs modernes, dans des temps où l'instruction se fait jour à travers tous les obstacles, pour ainsi dire, de la nature mème, d'espérer d'être dignes un jour d'admirer, d'égaler même leurs aisêtres?

Il ne faut pas croire que des peuples, dont l'énergie n’a besoin que d'être excitée pour exécuter de grrandes choses, feroient, de la liberté même, une arme funeste pour s'égorger. De ce qu'ils ont été les victimes du fanatisme, comme plusieurs peuples del'Europe, il ne s'ensuit pas qu'ils se livreroient encore à ses fureurs.

M. Paw, qui en rent à toute la terre, donne un coup de fouct en passant aux Miänotes; il les représente, et comme des brigands détestables, et comme des làches, dépourvus de toute valeur guerrière. Enfin, livré toujours à son imagination, il ne veut pas qu'ils soient désormais les descendans des anciens Spartiates. Il accuse leurs Caloyers d'être des fabricateurs de faux titres et de fausses généalogies.

Nous ne prétendons pas suivre M. Parr dans sa marche hasardée; nous dirons seulement que - es Mainotes, pour avoir résisté jusqu'ici à tous , es efforts des 'Turcs, ne sont ni deś brigands, ni des rebelles; ils défendent uniquement leur 
148

liberté. A-t-on jamais donné aux habitans des montagnes du Chili, cette dénomination odieuse, pour avoir osé défendre la leur contre les Européens? Mais, dit-on, les Mainotes sont les pivates de la mer. Rien n'est plus rare: ce n'est quechez les Cacovongnotes, qui forment une tris-petite peuplade de cette contrée, qu'on en trouve. Ce nom de Cacorougnoles vient de $\mathbf{C a -}$ cosonnia, qui signife montagnes d'mu accés difficile; ainsi il appartient anx habitans de ces montagnes. Il a plu cependant à M. Paw de le tradure par les scélérats des montagnes.Commeson impartialité règne dans les plus petites choses!

Au surplus, si les Mänotes courent effectivement sur les vaisseaux de toutes les Puissances, ils doivent en être poursuivis à leur tour, et une pareille gुerre cesseroit bientôt, si quelque grande Puissance, comme la France, engagrcoit la Porte à reconnoître pour liépublique souveraine, cette petite partic de la Morée, sur le pied de celle de Raguse, c’est-à-dire, tributaire de la Porte; alors les Mainotes s'adonne. roient au commerce et à la navigation, et bien loin d'incommoder leurs voisins, ils leur deviendroient utiles.

Les Mainotes, ct tous les Grecs en général 
SUr l'îte de Syra. Chap. VIII. I4) ainsi que nous l'avons vu dans le chapitre préé́dent, ont pour le métier des armes, les mîmes dispositions que les Turcs de l'Europe (I): même bravoure, mêne courağe, même impétuosité. Tout le monde convient quil ne manque aux troupes ottomanes, que la discipline miJitairc, pour être les meilleures de l'univers: il en seroit de même des Grecs, sills étoient exercés. Lorsque les Russes descendirent dans l'Arehipel, ils entreprirent d'en enrégimenter plusieurs, et ils furent étonnés de la facilité avec laquelle ils apprirent leurs évolutions. Les Officiers pensoient qu'il falloit beaucoup moins de temps, pour en former de bons soldats, que pour d'autres nations, des Russes mêmes. Le P.Sauger, dans son histoire des

(I) Cela est si vrai,que les Turcs d'Europe peurent passer pour des Grecs. Depuis près de 400 ans, qu'ils habitent ces contrées, qui ont produit tant de héros, ils doivent avoir hérité de leurs dispositions pour la guerre. Aussi les autres Souverains de l'Orient, sur-tout ceux de I'Inde, regardent le Grand-Seigneur, comme Empereur des Romains, c'est-à-dire, des Grees. Ceux-ci, depuis la translation de l'Empire Romain a Constantinople, s'appellèrent en gree, Рopesis Romaii ; en ture. oustums, qui veut dire romasins.

K iij 
1 วั้ PRÉCIS HISTORIQUE

Ducs de l'Archipel, rapporte des prodiges de valeur que ces Princes ont fait avec despoiguées de Grees.

Enfun, M. Paw est si acharné contre les Maïnotes, qu'il vondroit les faire passer pour antropophages, et mettreen doute ce qui est connu de toute la Grèce, je veux dire, la générosité que ce peuple libre exerça envers tous les Princes qui se réfugièrent à Maïna, après la destruction de l'empire d'Orient. Les Paléologues, les Lascaris, les Phocas, les Cantacuzénes, trouvèrent chez ce peuple un asyle aussi sûr que désintéressé, et les descendans de plusieurs de ces princes y existent encore. Le dernier qui arriva à Maïna fut Nicéphore Comnène, fils de David, dernier Empereur de Trébisonde. Ce Nicéphore y fut non-sëulement accueilli par les Mänotes, mais encore proclamé par eux Prologeres, ou premier Sénateur. Quoique M. Paw ne puisse pas douter lni-même de l'existence de Nicéphore, ni de son arrivée à Maïna, il voudroit néanmoins, environ 400 ans après, le faire passer pour un aventurier. Cette accusation est démentie par une foule de titres qu'on ne peut pas révoquer cn doute; et d'ailleurs cet auteur n'uuroit-il pas dû roir l'inyraisemblance de son 
SUR L’̂̂le DE Syr A. CHAp. THII. I5I assertion daus ce seul fait? c'est que tous ces Princes réfugiés, dont nous venons de parler, étoient les oncles, les cousins, les parens, plus ou moins proches de Nicéphore; et s'ils nel'eussent pas connu pour être le fils de David, n'estil pas hors de toute probabilité, qu'ils se fussent joints aux Maïnotes pour lui acorder la prééminence sur eux, en le proclamant unanimement Prologeros, dignité qui a été depuis héréditaire dans sa famille et que ses descendans ont conservée jusqu'à nos jours?

D'après ces faits incontestables, rien n'est plus naturel que l'existence des titres, qui constatent Ia filiation de Nicéphore, et en falloit-il d'autrés que les actes de mariages et de baptêmes, trouvés à Maïna et sans aucunes lacunes, dans un si grand laps de temps? Où est donc cette quantité prodigieuse de titres que M. Paw reproche aux Calorers de Vitulo, et aux autres Moines de la Grèce, d'aroir forgés, et qu’à peine la vie d'un homme suffiroit pour les lire? Il avoit onblié qu'il aroit dit plus haut dans le même ourrage qu'on ne tiouvoit pas un scill manuscrit en Grèce, et que 'les Grees modernes sont si ignorans, qu'ils ne se doutent sculement pas de ce quils ont été. Avouons, cependant $\mathrm{K}$ iv 
que pour forger certains titres, il laut avoir me certaine éducation; car comment parviendroiton à combiner, à lier une quanticé de dates, de faits, etc.? M. Paw a-t-il pu imagriner que les Grees aient jamais songé à fabriquer de faux titres et defausses généalogres, sous un gonvernement qui a poursuivi, comme criminels, crux qui portoient un grand nom? Cette persécution a réduit ou contrant les Grees illustres à caller leur naissance et leurs titues, et à chatuger de nom; ce qui a occasionné la perte des titres de tant de familles qui oni jadis figuré en Giece, et qui existent anjourd'hui, en plus grande parie sousdesnoms inconuus. D'après cela spurl, ceux qui ont lu M. Paw, jugeront facilement combien il est en contradiction avec lui-même, et seront moins surpris quilil acccuse les Maınutes d'être des brigands, et Nicéphore un aventurier, puisquil accuse également les Spartiates de cruauté et de brigandagre, et Lycurgue d'être un législateur supposé.

Enfin, l'après l'assertion de M. Paw contre le précis historique des Comnènes, il semble. qu'il ignore qu'une branche cadeıte de c'es mêmes Comnènes de Tr ébisonde, est élablie, depuis plus de deux siècles, à Chambéry, et que les Ducs de 
SUR L'île DE Syra. Chap. VIIT, I 53 Savoie ont toujours reconuu l'illustre origine de ces Comnenes, dont plusieurs princes de ce nom et de cette branche ont été accueillis dans tous les temps, par différens Souverains, tels qua les Empereurs d'Allemagne, les Rois de France, Henri III et Henri IV. 


\section{H A P I T R E I X.}

De la langue des Syriotes et des autres Grecs modernes.

L A langue des Syriotes, celle de tous les habitans cles îles de l'Archipel, et des Grecs du continent, est la véritable langue grecque. Sa prononciation est la plus conforme à celle des anciens; ce n'est pas celle de quelques savans de Paris, qui portent la prétention jusqu’à vouloir nous l'apprendre.

Les langues ont varié plus ou moins chez tous les perples; de vieilles expressions ont dis. paru, de nouvelles ont pris leur place; la prononciation a éprouvé même des changemens ; mais il faut avoner que sil reste encore des traces d'une langue ancienne, c'est dans les contrées ou elle a jetté le plus d'éclat, qu'il faut aller les chercher, et non chez les étrangers. 
SUR L'ÂLE DE SYRA. ChAP. IX. T55

Unsavant Elléniste de Paris, à qui je faisois part un jour de ces réflexions, me dit que cela étoit vrai en général; mais quä l'égard de la prononciation de la langue grecque, la multitude des Barbares, qui aroient inondé la Grèce, avoient aussi corrompu sa langue. Je lui ciemandai quels étvient ces Barbares? les Gaulois, me répondit-il, qui, sous Brennus, ravagèrent l'Autique, brulèrent le temple de Delphes, passèrent en Asie, et y fondèrent le petit empire appellé la Galatie; mais si, répliquai-je, une poignéc de Gaulois, en traversant la Grèce, a pu corrompre sa langue, comment se pourroit-il qu'en passant dans les Gaules elle ne s'y fût pas altérée?

Ni l'inrasion de ces Barbares, ni celle des Perses ne corrompirent la langue Grecque; elle fut au contraire plus florissante que jamais sous la domination d'Athènes, et sous l'Empire d'Alexandre, elle l'étoit même sous la Puissance des Romains. Les Chrisostômes, les Baziles, les Grégoires de Nazianze, et une foule d'autres écrivains, aussi sublimes qu'élo. quens, attestent cette vérité.

Les Turcs, dira.t-on peut-être, en subjugant tout l'Empire Grec, ont aussi corrompu sa 
langue ; mais je répondrai que s'ils sont parvenus a faire adopter quelques mots aux vaincus, que sils en ont Grécisé quelques autres, il n'ont pû faire changer la pronunciation. II auroit fallu pour cela que les conquérans se fussent allićs ou mélés avec erix; qu’ils eussent appris et parlé leur langue, ce quils nont jamais fait. Après leur conquête, ils ont élevé entre eux et les Grecsun mur de séparation, q̨ui dure encore. Habitans des mêmes contrécs ft des mémes villes, les Grecs ct les 'Turcs ne sont pas plus liés ensemble, que les Peuples du nonveau et de l'ancien continent.

Ce qui me filit croire encore que la prononciation de la lansize grecque n’a point dégénćré, comme les Ellénistes François le prétendent, c'est que tous les Grees nodernes n'ont qu'un sentiment sur ce point. Déjuis les bords du Nil jusquà 'Trébisonde, et du Golle Adriatique jusqưà la Criméc, les Grees qui habitent, ou les côtes, ou l’intéricur du pays, parlent grrec, et ils prononcent tous de même : cela pourroitil. ĉtre ainsi, si la prononciation de la langue grecque avoit été corrompue, et si elle nétoit plus ce qu'elle étoit autrefois? Les Melchites de la Palestine, qui n'ont aucune communication 
SUR L’île de Syra. Cha P. IX. I57 avec les Grecs du Levant, et qui partent une langue si différente de la leur, ont la liturgrie «recque, et le bréviaire grec; et depuis Saint-Basile et Saint-Clrysostôme, ils récitent et proroncent comme les véritables Grecs. Enfin les descendaus de ce peuple, émule et rival des Grces, n'auroient-ils pas reproché à ceux-ci, à quelque époque la corruption de leur langue? On ne trouve cependant mulle trace quils leur aient jamais fait ce reproche : il y a au contraire entre cux une parfaite harmonie à cet égard.

La langue grecque a tonjours été très-donce et très-harmonieuse, mais les Ellénistes de Paris la prononcent d'une manière si monstrueuse, quil n'est pas possible de l'entendre: veulent-ils prononcer, par exemple, les hommes ot les fentmes, ils diront: ö̈ antropoï laï ä̈ gunaiikes; nous dirons nous, i anthropi k'e ghinckes: lessavans jugerout lequel desdeux a plus d'harmonie.

Personne n'éloit plus attaché à la manière de prononcer la langue grecque comme l'université de Paris, que M. de Villoison de l'acadénie des inscriptions et belles-lettres; cependant après avoir voyagé en Grèce, après avoir vécu et conversé avec les Grecs, il a été obligáde con- 
venir que l'université de Paris étoit dans l'clreur sur cette prononciation : son oreille s'étoit accoutumée à la douceur, à l'harmonie de celle des Levantins : il ne pouvoit plus l'entendre parler en France : il en a été de même de tous ceux, qui, sans prétention et de bonne foi, ont parcouru nos contrées, ct n’ont cherché qu'à s'instruire. Quand ils ont comparé notre prononciation à celle de l'université, ils n'ont pas hésité de préférer la première et de l'adopter comme la seule vraic et conforme à celle des anciens.

Mais de quoi s'agit-il ? c'est de savoir s’il faut prononcer certaines consonnes et certaines voyelles comme les Grecs modernes, ou comme les El. lénistes deFrance.Ceux-ci prononcent le $\gamma, \delta, \theta, \chi$, $s, d, t, c$ comme dans leur langue; les Italiens aussi, faute de dispositions dans les organes, les prononcent de même; mais nous les prononçons au contraire, comme $g h$, $a h$, $t h, c h$. Les Italiens conviennent cependant qu'il $y$ a plus de douceur et de mollesse dans notre manière, et pour cela ils avouent que notre prononciation à cetégard, est celle des anciens. Les Savans de France, à ce que je me suis apperçu, ne font pas de difficulté de nous accor- 
SUR L'îlE DE SYRA. ChAP. IX. 159 der cette grace ponr ces mêmes consonnes, mais non pour les voyelles. Dans celles-ci, parexemple, $a t, \varepsilon r, o r, x, v$, ils prétendent qu'il faut prononcer les trois premières, comme si elles étoient deux lettres $a \ddot{i}, \varepsilon \ddot{i}, o \ddot{i}$ : à l'égard des deux autres, la première conme $c$, la seconde comme $u$; nous prononçons au contraire la première comme $\varepsilon$, les quatre autres comme $i$, et c'est ainsi que les anciens Grecs les prononçoient.

Dans un petit ouvrage intitulé, Hadriani Relandi dissertationcs miscellanœ, imprimé à Utrecht en 1708, on en trouve des preuves décisives. C'est à la page 157 de la troisième partie: il faut cependant être prévenu que l'auteur avoit adopté la prononciation des Ellénistes de France, et qu'il sembloit ignorer que les Grecs modernes ne prononçoient pas comme lui. L'ع łes Grecs ne se prononçoit pas anciennement comme aujourd'hui, puisque, selon le té-

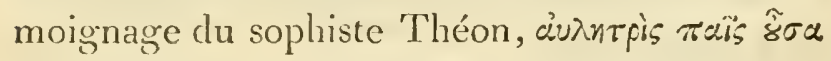

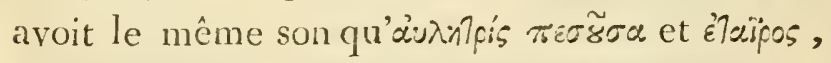

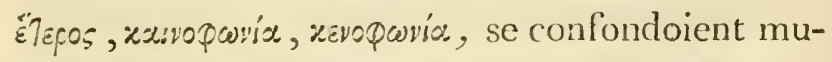
tuellement, et on prenoit un mot pour l'autre: l'és et l'ravoient anciennement la même signification; aussi P. Nigidius prétend que le mot latin 
amici s'écrivoit amicei au nominatif pluriel, pour le distinguer d'amici au génitif, quoiqu'il s.e prononçât toujours de même. Cicéron (lib.9, epist. 16. ) nous dit que le mot grec Rivé convient avec le bini; aussi les Latins ont-ils formé

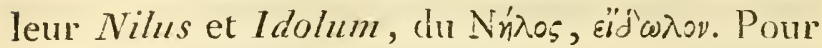
ce qui est de l'n, Eustathe a écrit que Nebrev's

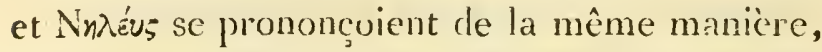
mais qu'ils s'écrivoient différemment. L'or avoit aussi une autre prononciation chez les anciens que chez les modernes. En effet, 'Thucydide

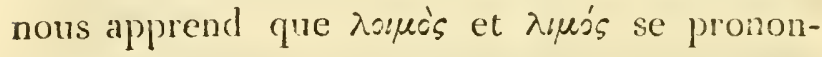
çoient de même, en nous rapportant cet oracle,

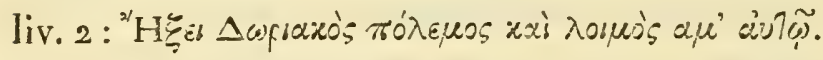
Il $y$ aura nne gucre avec les Doriens, el lie

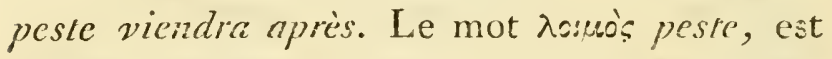
équivoque, et poutroit se prendre pour $\lambda$ biejs,

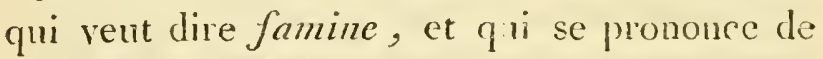
même. Sidonias Apollinaris aussi prononcoit le mot roïas misas.

Ter denas tropico prope currere climate mirizs.

Voici le texte la tin : $\dot{E}$ gracomum oporter alifer olim fuisse promuncialum, ac numc: qumm

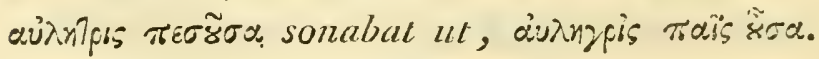


SUR L'îlE DE SYRA. CHAP. IX. 16: Teste Theone sophisia ( I ), el éteros étaipos, xeropariy xatrquwix inter se corfundebantur; n cum eq quondan conveniebal, quum P. Nigidius (2), tesle Gellio (3), 13, if annolabat aliler scribi amici in nominativo plurali, scilicet amici, uliter in genilivo singulari amici; quum Cicero (lib. 9, epist. 16.) Fivé convenire cum bini scribebat: quum ex Néìos Nilus, घífwiov idolum, clc. orium traxere. Aliker quoque $\tilde{\dot{y}} \mathrm{~s} \%$ ac nunc pronnnciatum fuit quando Eustallius (4) scripsit Neinè's consenire suno

(1) Cet auteur est avantageusement connn dans le monde littéraire par un traité de rhétorique.

(2) Publius-Nigidius, bon humaniste, habile philosophe et grand astrologue, passa pour le plus savant des Romains apres Varron. Il mourut l'an 45 arant J. C. Ciceron fait de lui le plus grand éloge. Il ne nous reste de ses écrits que des fragmens.

(3) Aulu-Gelle, Aitlus-Gellius, grammairien 1atin, florissoit à Rome, sa patrie, vers l'an 130 de J. C. Il publia un ourrage en ringt livres, intitulé : Les Nizuts Attiques, qu'il nomma ainsi, parce qu'il l'aroit composé à Athènes pendant les longues soirées de l'hiver. Cest un recueil de beancoup de matières différentes. 11 peut serrir à éclaircir les monumens et les écrivains de l'antiquité : on y troure quantité de fragmens dez anciens auteurs.

(4) Eustathe, Eréque de Thessalonique, dans le Tome $I$. 
162 PRÉCIS HISTORIQUE

cum Nn入éus, non vero scriptione. Diverso etiam

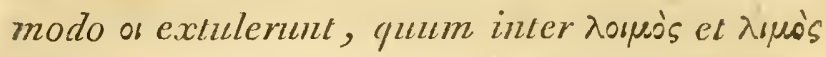
vix ulla differentia audiebatur, quod Thuicy. didis nolat, oraculum hoc narrans, lib. 2,

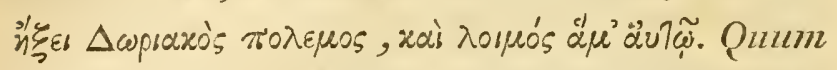
grecum roipas Sidonius Apollinaris miras promunciabat c. I5. Ter denas tropico currere climale miras.

A tout ce que nous venons de dire, on peut ajouter ici pour derniere preuve, qu'on rencontre chez les anciens auteurs et dans les dictionnaires grecs, quantité de mots qui ont les diphthongues $\alpha b, \varepsilon b, a b, o u$, etc. surmontées de deux points, comme aïfpus-eos, ignorant; aï 'w-

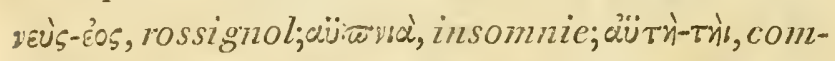

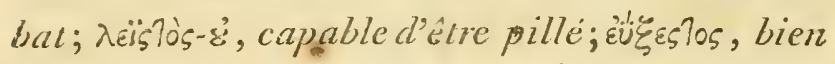

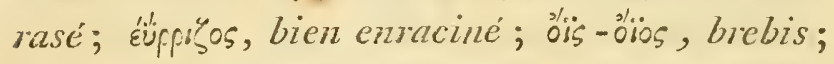

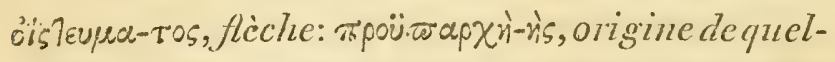
que chose; троӥшеруабia, préparation, et plusieurs autres. Dans tous ces exemples, aï, aii $\ddot{i}$, ö, ö̈, ne sont pas sensés diphthongues, ils

XII ${ }^{e}$ siècle, étoit un habile grammairien; il laissa des commentaires sur Homère, ct sur Denys le géographe. Son travail sur la poésie grecque est fort étendu ot très.estimable. 
SUR L'île DE SYra. ChA P. IX. 163 doivent être prononcés séparément comme deux voyelles.

Si donc il est vrai, d'aprés cet axiome, que l'exception confirme la règle, on doit croire que toutes'les fois que ces voyelles ne sont pas marquées ainsi, il faut les prononcer comme nous l'avons dit ci-dessus, c'est-à-dire, comme diphthongues; excepté que l'as et l'eu, dans les derniers cas, on doit les prononceren $\nu \dot{e}$, c'est-àdire, comme un $\nu$ consonne; aussi nous disons

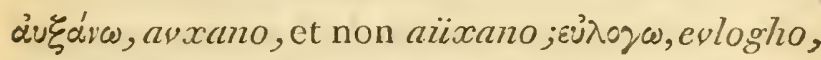
et non eïlogho, comme les Hellénistes françois le disent.

Avant d'aller plus loin, je veux répondre à quelques difficultés que nos adversaires opposent quelquefois. Ils disent que l' traduit, par les anciens écrivains romains, en e. Cela est vrai ; mais il est vrai aussi que les anciens traduisoient toujours l'ar par une diphthongue $a$ ou $e$, $\varepsilon$ par un $i$, or par un $a$, etc. et jamais ils ne lesséparoient, comme les savans de Paris le prétendent. A joutez à cela, qu'il semble que les anciens prenoient, au moins dans quelques circonstances, l'e pour $i$;'ainsi nous trouvons écrit domines pour dominis au datif plurier. En second lieu, un ancien écrivain, Denys 
I64 PRÉCIS HISTORIQUE d'Halicarnasse, assure que les Grecs prononçoient l'u $\psi \wedge \lambda$ ov en arrondissant la bouche. Je puis arrondir la bouche en prononçant l' $i$, et si l'on n'en convient pas, je dis que, strictement parlant, on n'arrondit la bouche qu'en prononçant l'o. Il falloit donc, d'après cette autorité, prononcer l'v comme o. Avouons donc que de semblables raisons ne sont pas décisives, et ne peuvent affoiblir celles que nous ayons rapportées en faveur de notre opinion.

On nous oppose encore la fameuse autorité de Cralimus, qui dit que les Grees imitoient le bêlement des moutons, en prononçant le $\beta$. Il faut, disent-ils, le prononcer beila, et non vila, comme les Levantins. Une Dame de Constantinople, établie à Paris, disputoit un jour sur la prononciation de la langue grecque, avec un savant Helléniste de cette capitale, qui cita l'autorité de ce cri des moutons; la Dame quitta aussitôt la conversation, en s'excusant de ne pas entendre le langage des bêtes.

Quautàmoi, jai fait plusieurs fois attention au cri desmoutons, et j’ai observé qu'il étoit im. possible de distinguer sils exprimoient beh, veh ou meh. Les Levantins turcs et grees rendent ce cri par le meh; aussii les Turcs 
SUIK L'ille DE SYRA. Chap. IX. I65 l'appeilent-ils memclek. Nos ant agonistes ne peuvent donc rien gagner àcette autorité.Il faut qu'ils s'en remettent entierement au jugement des Grees modernes, sur la prononciation de leur langue, comme les autres nations doivent s'en rapporter aux François, sur la prononciation de la leur.

Enfin, si les Grecs modernes ont changé la lettre beia en vira, cela n'a pu avoir lieu que par la communication qu'ils ont eue arec les peuples qui la prononçoient ainsi, faute d'aroir la lettre $\beta$; ct comme ceux que les Grecs ont le plus fréquentés, sont les Italiens et les Turcs, qui tous ont la lettre $b$, car les premiers prononcent encore dans leur langue Basilio, et non $V$ asilio, Barca, et non Varca, comme les Grecs, il n'est pas vraisemblable que la lettre $\beta$ ait éprouvé la moindre aitération par la communication des Grecs avec les nations étranğères.

Venons à l'autre proposition, et voyons si la langue que l'on parie à Syra, et dans le reste des îles et du continent de la Grèce, est une langue grecque. Sil faut en croire plusicurs royagcurs et quelques savans, le grec d'aujourd'hui n'est qu'un produit monstrueux du 
beau grec ancien, auquel même il ne ressemble presque plus.

Javoue que la langue des Grecs modernes n'a pas'la richesse de celle des anciens, qu'elle est infiniment moins élégante, et beaucoup plus facile; mais elle n'est pas moins une véritable langue grecque, qui nous a été transmise par les anciens Grecs; et voici à cet égrard ce que nous จิvons à dire.

La langue d'Homère, de Démosthène et d'autres Grecs célèbres, telle que nous la voyons dans leurs écrits, étoit infiniment riche en synonymes: la culture des sciences et des belleslettres étant malheureusement tombée, sur-tout après la chûte de l'empire, parmi les Grecs modernes, ils en ont conservé quelques-uns, dont on fait usage communément, mais ils ont presque entièrement oublié le restc. Il ne faut pourtant pas simaginer que notre langue moderne soit entièrement dépourvue de synonymes; pour exprimer seulement l'action de voir, nous avons

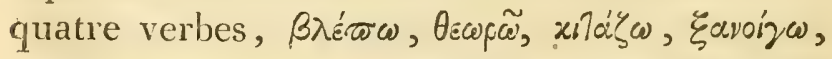
je vois; et un Grec quelconque, pour peu qu'il s'adonne aux lettres, son langage s'épure, et devient plus riche et plus fécond.

Les anciens se servoient avec beaucoup de 
SUR L'îlE DE SYra. Chap. IX. 167 grace des participes, des adverbes, des particules et des prépositions. Les Grecs modernes n'ont plus ces tournures heureuses, ccs transpositions hardies, qui changeoient quelquefois le sens des phrases. Cette élégance suppose la culture des lettres.

Dans les anciens Grecs, on voit une quantité de temps, d'aoristes, de parfaits, de plus que parfaits dans tous les modes. De plus, outre le singulier et le pluriel, on voyoit aussi le duel. Les modernes ne connoissent point cette multitude de temps ni ces duels; ils n'emploient dans leurs discours que le présent, l'imparfait et l'aoriste second qui est le parfait. Quand ils veulent former le plus que parfait, ils se servent diz verbe auxiliaire ëzo, j'avois, comme les Francois et les Italiens; pour j'asois aimé, ils di-

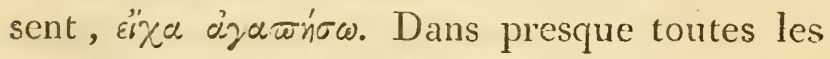
langues, on peut énoncer de deux manières, linfinitif et le futur. En latin, par excmple, volo ut manduces, et volo te manducare, ne veulent dire autre chose que, je veux que $\mathrm{lu}$ manges; volo manducaré et manducabo veulent dire, je mangerai. La même chose étoit observée par les Grecs anciens. Les Grecs modernes ne se servent point des infinitifs simples, mais 
pour énoncer un temps infini, ils emploient la

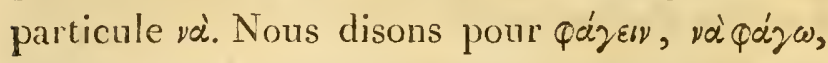
manger. Notre va' est le même que le iva' des anciens; l'ut des Latins est le que des François. Pour former le futur, nous nous servons toujours du verbe $\theta \dot{\hat{\lambda}} \lambda \omega$, je veux $x$ ainsi nous disons

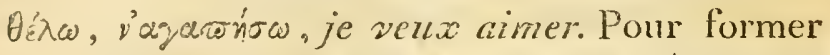
l'optatif, les Grees modernes se servent de l'adverbe $a_{s}^{\prime \prime}$, qui étoit chez les anciens un adverbe,

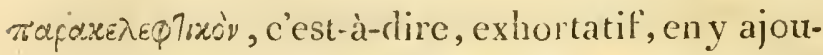
tant le $\sigma$ par uae figure, zpooxnialorios, proschématisme; de même que par une aphéraise, nours ôtons le r à l's $s \alpha$ ', et nous formons notre $\nu \alpha$ '.

On doit rapporter à ce que je viens de dire, Ia cause de ce que les Grees modernes n'entendent pas parfaitement les auteurs anciens, tels qu'Homère, Démosthène, Thucydide. Malgré cela, ils recomnoissent presque la moitié des mots, parce qu'ils sont les mêmes que ceux dont on se sert actuellement, ou qu'ils ont avec cux beancoup d'analogic on de liaison. D'ailleurs, presque toutes les phrases des Grecs modernes sont comme celles des anciens, et elles ont la même signification; et si les modernes leur en donnent quelquefois une différente, cela ne diminue point le prix de la langue actuelle. 
SUR L'île DE SYRA. ChAp. IX. I 69 On observe aussi qu'il y en a plusieurs qu'on ne trouve point dans les ouvrages des anciens; cela n'empêche pas que le mot ne soit véritablement grec.

En effet, les Grecs modernes emploient familièrement une infinité de mots qui sont les mêmes que ceux des ancicns; tels sont, par

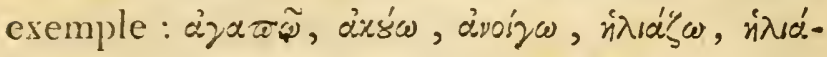

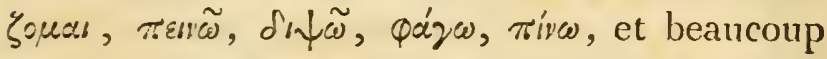
d'autres. J'aime, j'éconte, j'ouvre, j'cxpose, je m'expose au soleil, j’ai faim, jai soif,

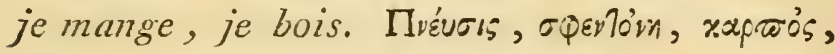

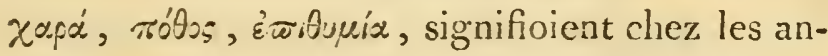
ciens, ce qu'ils signifient chez les modernes: Respiration, fronde, fruit, joie; les deux derniers mots, desir. Ils emploient aussi presque toutes les prépositions et les particules des an-

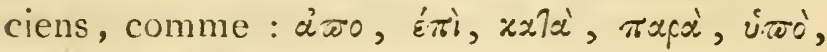
vi.̄ép, हiv, ivx, ws, etc.

Il $\mathrm{y}$ a encore chez les modernes plusieurs mots qui, avec l'apparence d'être grecs, ne se trouvent cependant nulle part chez les an-

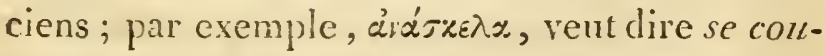
cher sur le dos; rópuía, concher avec le visage

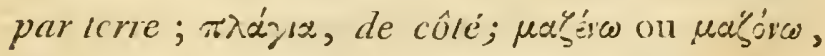

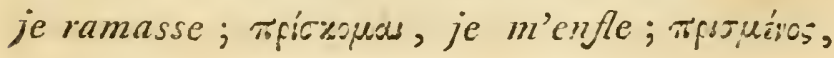




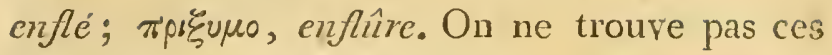
mots, dans les Dictionnaires, cependant ils ont tous le caractère grec.

Il y en a d'autres qui, quoiqu'on les y rencontre, ont une signification différente. Par

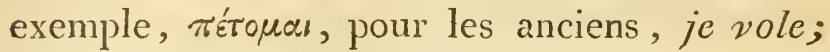
pour les modernes, il veut dire, je me fie. Ils.

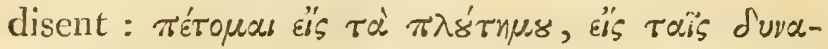

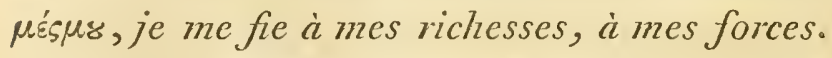
Les anciens se servoient du verbe słeqrów, pour couvrir ou restreindre, et nous pour sécher, et même proprement pour l'asciutare des Italiens. Icíros chez les anciens, veut dire, mm chemin baltu: nous le prenons nous pour le fondement de l'homme, des animaux, et de toute autre chose. Nósturos, n, ov, exprimoit, pour les anciens, une chose qui pouvoit revenir; pour les modernes, une chose qui a de la grace et du goût. Nous disons aussi d'une personne, qu'elle est vós7un, c'est-à-dire, qu'elle a de la grace; d'un mets qui a bon goût, nous disons qu’il est vós7uro; de là vient

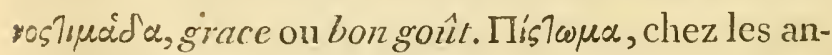
ciens, signifioit argument, preuve; nous entendons par là, au contraire, une position avec le visage par terre. Пésspa, pour les anciens, étoit la confiance d'entreprendre quelque chose; pour nous 
c'est obstination. Ces différentes acceptions ne doivent pas diminuer le prix de la langue moderne, ni la faire passer pour une langue corrompue.

Voici comment il convient de raisonnerlà-dessus : la langue françoise n'est pas'si riche en synonymes et en expressions que la langue grecque : nous avons dix mille fois plus d'ouvrages écrits en françois, que nous n'en avons en grec ancien; cependant il est à présumer que plusieurs mots françois en usage dans le discours familier, et plusieurs sens qu'on donne communément à tous les mots françois, ne se trourent pas dans toute cetre immense quantité d'ourrages; on peut dire la même chose de toutes les autres langues. Aussi voyons-nous que toutes les fois que l'on fait une nouvelle édition de quelque Dictionnaire, on est obligé d'yajouter certains mots nouveaux ou un nouveau sens à quelques mots qui y étoient déja.

La langue grecque des anciens étoit la plus variée de toutes celles qui ont existé. Ellese partageoit en quatre ou cinq dialectes principaux (I).

.(r) Parmi les cinq principaux dialectes de la langue grecque, il y en ayoit un qui s'appeloit le dialecte commun 
Chacun de ceux-ci se subdivisoit en phasieurs autres particuliers. Cette langue en général étoit infinment riche en expressions et en synony nes; elle leur donnoit différens sens, quelquefois

soit parce qu'il étoit composé, et qu'il contenoit les proprićtés desquatre autres, soit parce qu'il étoit d'un usage genéral, ou parce qu'il étoit enfin la base et la règle de tous les autres.

Les quatre autres sont le Dorien, l'Eolien, l'Ionien et l'Altique; roicil'origine qu'on leur donne. Hellen, surnomme Deucalion, fils de Jupiter, ayant occupé les pays de la Grece, ces peuples s'appelèrent Helleniotes, et leur langue helleniaque : il en sortit bicntôt cependant d'autres dénominations. Les fils d'Hellen s'ét ant dispersés en plusieurs contrées, les langues changèrent, et il se forma plusieurs dialectes.

Le Dorien tire sa dénomination de Doro; il s'établit dans les environs du Parnasse et d'Airope, et les habitans de ces contrées s'appelèrent Duriens. Ce même dialecte étoit en usage chez les Rhodiens, les peuples de Cnebe, du Péloponèse, les Siciliens, les Libyens et ceux qui habitoient l'ancienne Epire. C'est dans cette langue qu'Archite de Tarente a écrit, ainsi que 'Théocrite.

Eol, fils d'Hellen et de Pyna, donna son noin à un dialecte qui s'appela Eolicn, et qui s'établit dans les dix villes, et la province qu'il avoit fondées. Alcée a écrit dans ce dialecte. 
SUR L'île dE SYra. Chap. IX. I73 disparates aux mots. Outre cette richesse générale et cette variété, chaque dialecte en particulier avoit des phrases et des expressions à lui; et l'un donnoit sourent différens sens aux mots de

Ion, fils d'Apollon et de Creüse, fille d'Enachothe, fonda les treize villes le long des Cótes de l'Asie mineure, et qui composent la province Ionienne : leur dialecte en po:toit le nom, et c'étoit celui qui s'ap. prochoit le plus de celui des Athéniens. Ces treize villes étoient Clazomèues, Lebedos, Phocaia, Priène, Milet, Mycale, Erithra, Tios, Samos, Colophon, Ephésus, Chio, Smyrne. C'est dans ce dialecte qu'Hippocrate l'Tonien, Hérodote Halicarnassien, et Ho. mère, ont écrit. Ce divin Poète u'étoit pas cependant tellement attaché au dialecte Ionien, qu'il ne fit usage de tous les autres, et sur-tout de celui d'ithènes. On peut voir Plutarque à ce sujet : il cite différens passages d'Homère, où ce Poète s'est servi de tous les dialectes.

Le dialecte Attique étoit général à Athènes, et dans tout le district del'Attique. Il s appeloit ainsi, ou d'Atthide, fille de Crarai, Roi d'Athenes, ou parce que le pays étoit au bord de la mer, c'est-à-dire, littoral. En grec on dit rajaxrios, d'où on a pu former, en retranchant le $\pi \alpha \xi$, árziros. Thucydide, et Aristophane ont écrit dans le dialecte attique. 
I74 PRÉCIS HISTORIQUE

l'autre. Au milieu d'une richesse immense d'ex* pressions, avec une variété sans exemple de dialecte's, après une différence aussi multipliée de sens ne seroit-il pas possible que tout ne fût pas consigné dans le petit nombre d'écrits qui nous restent des anciens? Je conviens que plusieurs de ces expressions et deleurs sens même se sont perdus et oubliés, maisil faut avouer aussi que plusieurs autres auront pu se conserver parmi la nation grecque.

J'en suis d'autant plus persuadé, que si l'on vouloit y réfléchir sérieusement, on trouveroit que tous les mots et les sens que les Grecs modernes donnent à ceux qu'on ne trouve pas dans les écrits des anciens, en ont cependant les racines, et qu'ils ont entre eux quelque analogie. Deux exemples nous le prouveront.

Pour dire cnsemble, nous disons $\mu \alpha \zeta_{\boldsymbol{b}}:$ ce mot ne se voit nulle part dans les dictionnaires; mais on y trouve $\alpha^{\prime} \mu \alpha$, qui vent dire en-

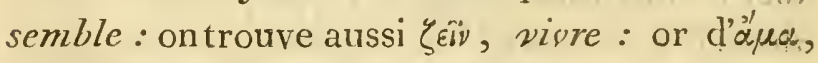

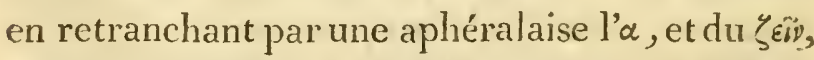
vient notre $\mu a \xi^{\prime}$ : tous ceux qui sont ensemble, respirent et vivent ensemble.

Pour dire à bonne heure, entre autres expres. 
SUR L'îLE DE SYRA. ChAP. IX. I7 sions, nous nous servons du vwris; ce mot, ne se trouve pas dans les écrits des anciens; cependant on y trouve "ै́v et ćco, d'où, par une

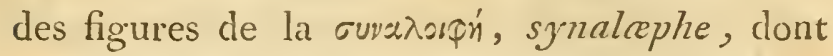
nous parlerons ci-après, se forme notre wapis; comme si l'on vouloit dire ếv ćpa, dans la minule.

On se tromperoit si l'on croyoit que les Grecs modernes donnent à tous les mots une signification différente, Par exemple, les modernes disent xвpóvw, je rends sourd; жצфútns, la surdité; et rвpòs, sourd. Les anciens se servoient de ce même verbe, de ce substantif et de cet adjectif, pour exprimer, alléger, légéreté , léger. Cependant notre zspóvw, etc. vient du

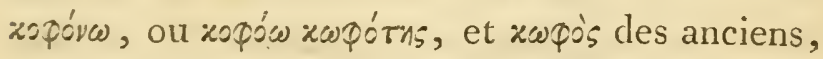
qui signifioient rendre sourd, la surdité et sourd, en changeant, par une figure gramma-

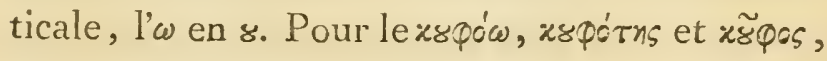
alléger, légérélé, léger des anciens, les modernes ne les connoissent pas, et ils se servent

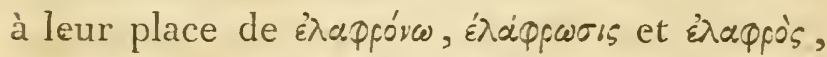
qui sont des mots aussi dont les anciens Grecs faisoient usage.

Il en est de même du verbe $\varphi \alpha^{\prime} \alpha_{\zeta}^{\prime} \omega$, que les modernes emploient pour dire, je ferme ou 
renferme; ce n'est pas le $\varphi r \alpha_{3}^{\prime} \omega, j e$ dis, je raconte, des anciens. Les premiers l'ont formé du $\phi p \alpha i \tau \tau \omega$ ou $\phi p c i ́ \sigma \omega$, en changeant les deux $\tau \tau$ ou $\sigma \sigma$, en $\zeta$, ainsi que les anciens le pratiquoient souvent. Eneffet, de notre $\phi p \alpha \zeta^{\prime} \omega$, nous formons le futur $\phi r \alpha \dot{\xi} \omega$, et l'aoriste $\ddot{\varepsilon} p \alpha \xi \alpha$, ainsi que les anciens de $q$ fá $\tau \tau \omega$; au lieu que du $\varphi \alpha_{\prime \prime}^{\prime \prime} \omega, j c$ dis, des anciens, le futur fait $\varphi p \alpha \dot{\sigma} \sigma \omega$, le passé tréppaxa.

Enfin, presque tous les mots grecs modernes, quoiqu'ils aient les mêmes significations que chez les anciens, sont cependant différemment écrits, soit parce qu’ils ont quelques lettres de plus ou de moins, soit parce quils ont changé une voyelle ou une consonne pour une autre. Cette variété n'étonnera point ceux qui connoissent la quantité des dialectes qui existoient anciennement parmi les différens petiples de la Grèce; et le grand nombre de figures qu'ils employoient dans les changemens de leurs mots. Or ces dialectes et ces figures sont la cause de la différence de plusieurs expressions des anciens et des modernes. Il y a cinq principales figures, que les Grees nommoient $\pi \lambda \varepsilon$ rararòs,

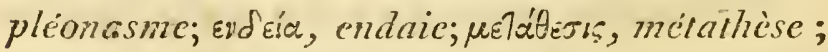

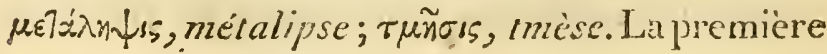


SUR L'ÂLE DE SYRA. CHAP. IX.

et la seconde de ces figures se subdivisoient chacune en neuf autres, qui s'opposoient entre elles. On peut les voir toutes chez Tryphon, grammairien auteur grec. Je rapporterai ici qu'une des figures subalternes de la

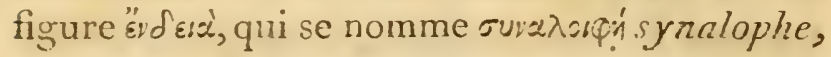
se subdivisoit en sept autres manières figuratives; trois simplés, $\mathrm{I}^{\circ}$. par élision, comme

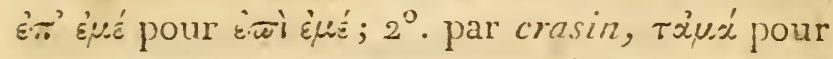

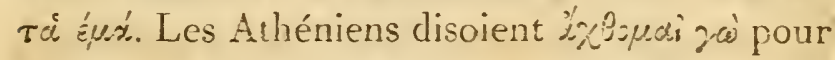

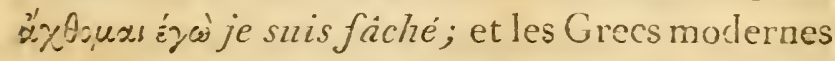

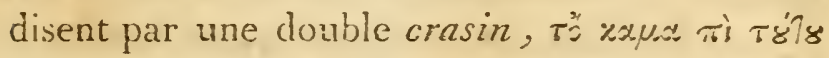

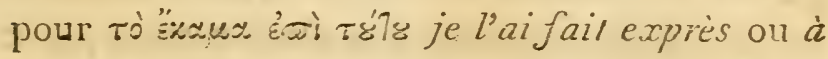
dessein; $3^{n}$. par synairesin, mâें हs porm mpriSis; et quatre composées, $1^{\circ}$. par élision et

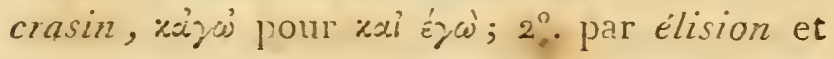

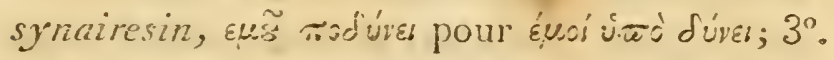

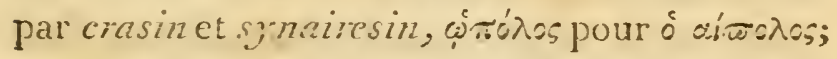

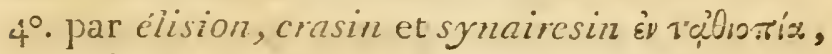

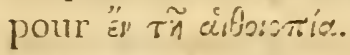

Nous devons rapporter à présent des exemples de la langue actuelle. Les Grecs modeines, par la figure prosthèse, lisent $\tau o^{\prime} \alpha^{\prime} \hat{s}^{\prime} \alpha^{\prime} \chi u$ épis, au lieu de ráxus, comme les Athéniens disoient autrefois ásłazus, ǹ pour exprimer la même

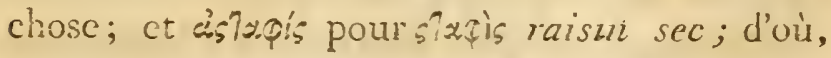

Tome $I$. 
I78 PRÉCISHISTORIQUE

par une autre figure, nous avons formé notre slapida qui veut dire la même chose. Ils disent aussi, comme les Athéniens, $\varepsilon^{\chi} \chi \theta \dot{s} s$ pour $\chi \theta \dot{s} s$ hier; ot dĩ $\lambda \hat{\varepsilon}^{\prime} \omega$ pour $\pi \lambda \varepsilon^{\prime} \omega$ nager. Ils disent éga-

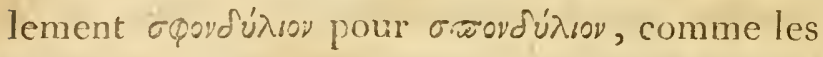
Athéniens disoient arsqápazos pour di.wápazos.

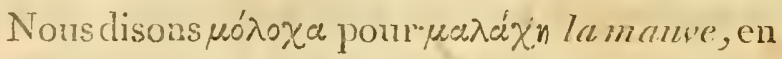
changeant les deux $\alpha$ en o comme les Athéniens; et l'nfinal en $\alpha$, comme les Doriens. Du mot ancien $x$ midn orlie, on a fait le notre arif'a, d'abord, en changeant l'n final en $\alpha$; ensuite, par une anadiriplose, nous ajontons le $x$, xnxúnfa. Enfin, par une troisieme figure, prosthèse, nous disons dixnzritina.

Les nodernes changent quelquefois les lettres aspirées en tenues, on celles-ci en aspirées;

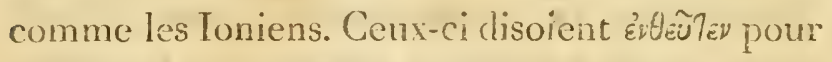

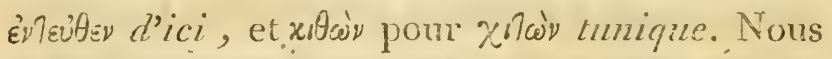

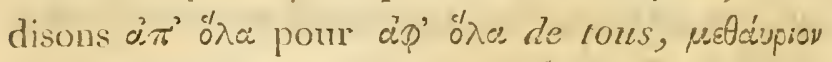

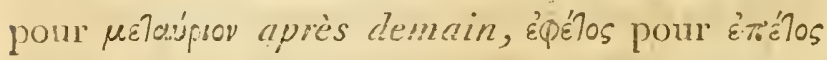
cetle ammé, et Bábpazos, ainsi que les Ioniens, porir $\beta a ́$ paxos grenouille; les Ioniens prononcoient aussi $\beta$ ófpaxos.

Ils changent aussi souvent les aspirées en d'antres aspirées, par exemple, фnxáps pour Qnxáp fourreau. C'est ainsi que les Éoliens ou 
SUR L'île de Sy R A. Chap. IX.

Doriens disoient prip pour Arip bête sanvage (d'où les Latins ont fait leur fera), et les Athéniens $\varphi \lambda \tilde{\alpha \nu}$ pour $\theta \lambda \tilde{\alpha \nu}$ briser.

De même ils emploient les lettres mouillées les unes pour les autres. Les habitans de

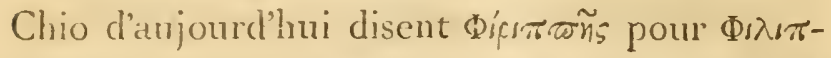

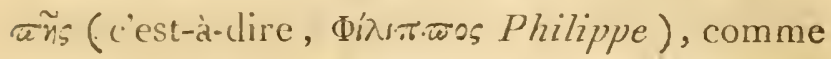

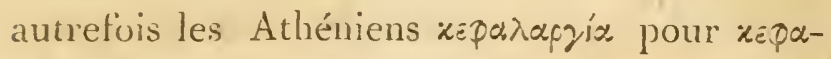

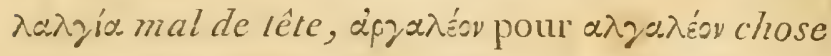
pénible, douloureuse. Les Grecs modernes disent éģalement $\pi \lambda \varepsilon \mu o ́ n$, comme les Athéniens

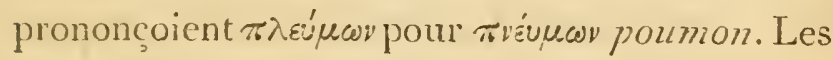
Latins ont retenu le changement de $v$ en $\lambda$ dans le mot pulmo.

Ils sippriment la première syllabe des mots, comme les anciens, par une figure appelée

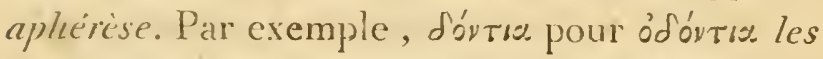

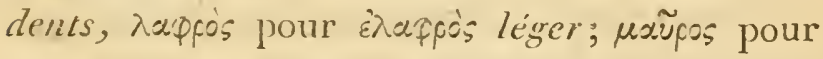

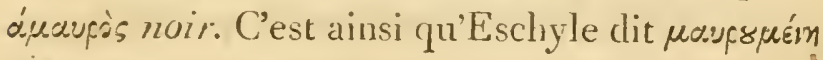

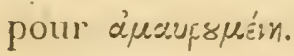

Les Ioniens aussi disoient óp'x̀ pour ćopiǹ fête ; xêives pour Ékêvos celui. Les Latins ont imité les Grecs, en retranchant l'o dans les mots dentes d'ơdón?es, nomen d'ovop.. Par la même figure, les Grecs modernes retranchent aussi les augmentations des prétérits, des participes; ils di- 


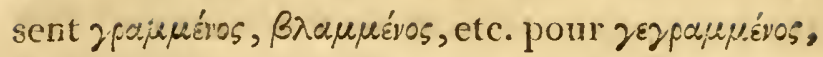

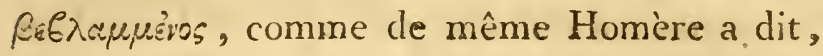
$\delta \varepsilon z \mu$ ćvos pour $\delta \varepsilon \delta \varepsilon \gamma \mu$ évos.

Ils retranchent une ou plusieurs lettres au milieu des mots; dans le premier.cas, le retranchement s'appelle ellipse, comme áłä pour l'ancien aífa vitte, sur-le-champ; ils disent aussi $\sigma \omega \dot{\pi} \alpha$ pour $\sigma \tilde{\omega} \pi \alpha$ raisé. Pindare a dit,

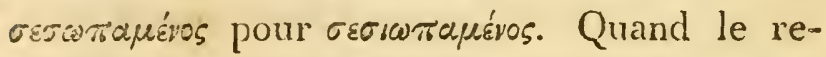
tranchement a lieu dans plus d'une lettre, ou dans une syllabe, il s'appelle syncope; c'est ainsi qu'on trouve dans Hérodote ỏ $\gamma \delta \omega^{\prime} r o v 7 \alpha$ pour özdońxovfa quatre-vings/s, d'où les modernes ont

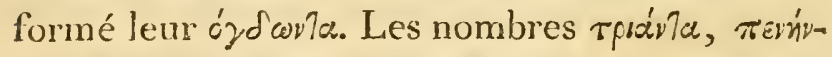

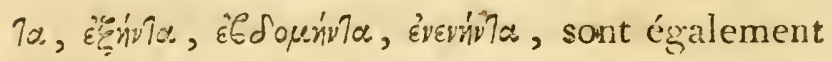

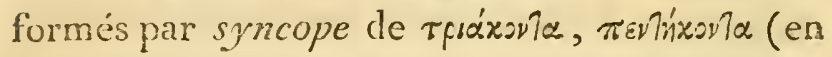

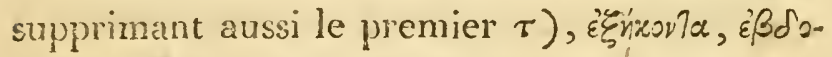

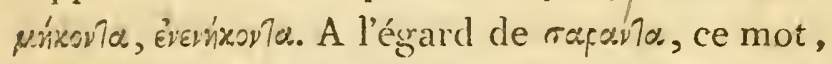

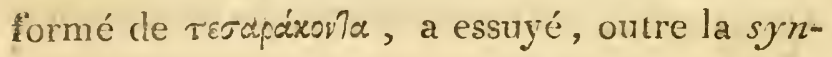
cope, ce que nous avons appelé plus haut aphérèse, ou retranchement de la syllabe initiale.

Une figure contraire à la syncope, c'est ce quion appelle épacièse, qui consiste à ajouter une lettre ou une syllable au milicu des mots. Les anciens Tarentins disoient $\hat{\alpha} \tau \omega$ pour $\hat{\omega} \tau \%$ ies oreilles; ils auroient dit, par la même ana- 
SUR L'île de Syra. Chap, IX. I8I logie, árie pour ẃriz, d'uù les Grees modernes

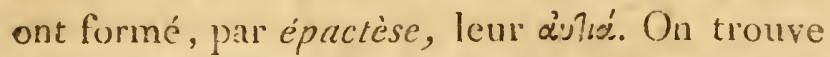

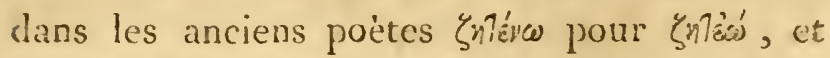

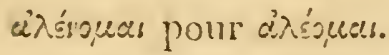

Quand le retranchement se fait à la fin des mots, il s'appelle apocope. C'est ainsi que les modernes furment une grande partie de leurs noms neutres, en disant ảylípour ảion oreille,

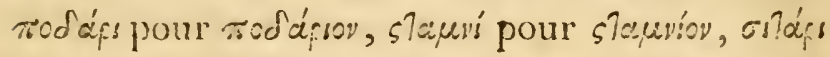

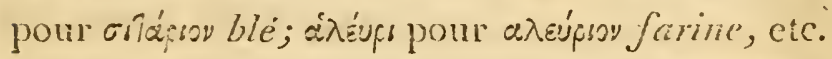
comme Homere a dit $\delta \tilde{\omega}$ pour $\delta \tilde{\omega} \mu x$ maison ou rerrasse; Hésiode $\beta_{p i}$ pour $\beta_{p} b$ i ; Empédocle

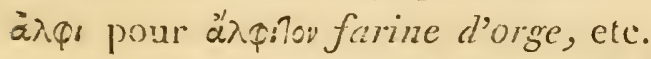

La figure contraire à l'apocope, s'appelle paragroge ou proschématisme, c'est-à-dire, prolongement; elle consiste à allonger les mots, en y ajoutant une syllabe à la fin. C'est ainsi

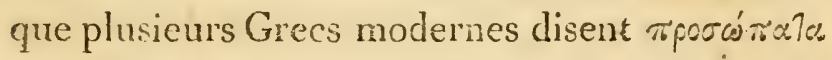

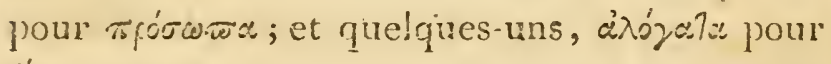
$\alpha^{2} \lambda$ o a cheraux. Les ancicns se servoient de vxix pour voi oui, et d's' $\chi$ i pour \& non. Les modernes cmployvicut pour ce dernier ó $\chi$, en retranchant l'u, et vairze et v'śs pour vaíx.

Ils connoissent aussi et emploient dans les discours toutes les autres figures; ils se servoient dia Tispò pour tofoinò matin, en faisant la contrac- 
182

PRÉCIS HISTORIQUE

tion d'، comme les Athéniens disoient $\pi f \omega^{\prime}$ pour zpwi, et la transposition de la lettre $p$, comme

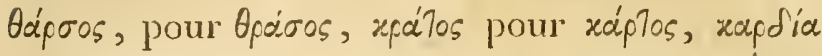
pour xpadia. 'Ils emploient les changemens des lettres comme les anciens Doriens, rpuфa' pour

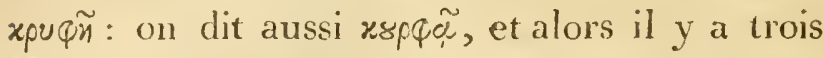
figures grammaticales, $I^{\circ}$. la transposition de l'p, dont nous avons parlé plus haut. $2^{\circ}$. Le changement de l'ý en $\alpha$, dont nous parlons. $3^{\circ}$. Par une figure nommće parenthèse, on y ajoute la lettre $o$, comme faisoient les Ioniens qui

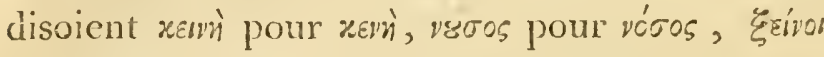

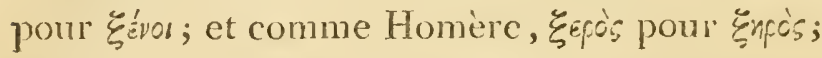

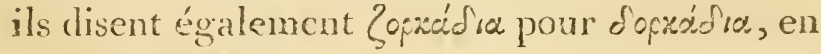
changeant le $\delta$ en $\zeta$ (comme les Doriens qui em-

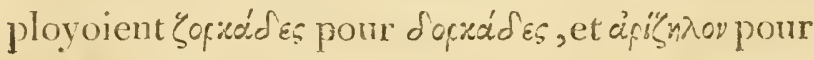
áfídnior), et l'o en a, comme les mêmes Do-

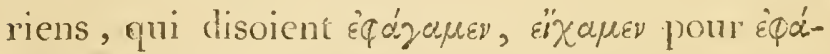

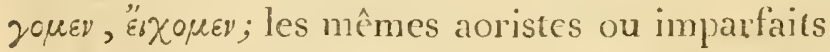
doriens ont encore licu chez les molernes; its

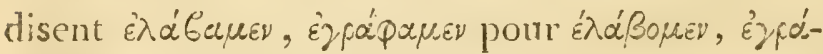

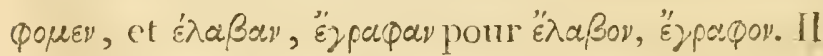
yen a aujourd'hui qui pronuncent árxmess pour

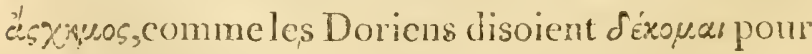
Séxoper, etc.

A la suite detontes ces difrérentes manières fi- 
SUR L'île DE SYRA. ChaP. IX. I 83 guratives de prononcer les mots, sont les changemens qu'on voit dans plusieurs des mots anciens et modernes, comme मं gépupa, nous dicons tò

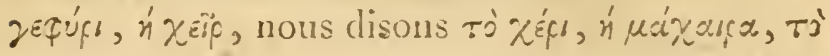
$\mu a \chi \sim ı f$, On ne doit pas s'étonner non plus de ce que les modernes changent aussi les genres des mots; cela s'est pratiqué souvent chez les anciens. Dans un dialecte, un mot se prononçoit comme masculin, et dansun autre comme féminin : le mot $\Delta \varepsilon \lambda \hat{\imath}$ ìv iros, étoit masculin chez les uns, et féminin chez les autres.

Enfin, dans tous les rerbes des anciens, terminés en ów, nous y ajoutons, par une de ces

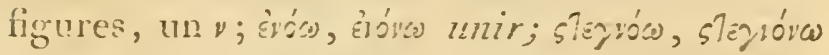
sécher; quierów, quveŕres décousvir, dévoiler;

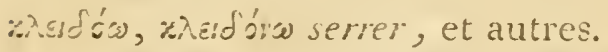

Si l'on est curieux de savoir comment du beau gree de l'antiquité, s'est formé le grec moderne, qui, nous en convenons, n'a pas la richesse, la grace et l'éloquence du premier, roici ce que nous en pensons.

Outre les cinq dialectes principaux, comme l'Tonien, l'Ĺlolien, le Dorien, l'Attique et le Commun, il y en avoit dans les difrérentes autres petiles contrées de la Grèce, une infinité d'autres qui en formoient comme autant de M. 
184 PRÉCISHISTORIQUE

subdivisions; ce qui constituoit une si grande variété dans la langue grecque, qu'on n'en a jatnais vu de semblable dans aucune langue de l'univers.

Il est encore très-probable que, dans les temps même où l'éloquence brilloit avec plus d'éclat parmi les Grecs, il y avoit, même à Athènes, un langage pour le peuple, qui n'étoit pas celui d'Homère ni de Démosthène. Les ouvriers, les gens de la campagne ne connoissoient certainement, et n'employoient pas daris leurs discours, ni les différens temps de tous les'modes, ni les duels, ni les tommures élégantes qui font un des plus grands mérites de la langue grecque. Cela est si vrai, que St. Jean-Chrysostôme, dans sa jeunesse, prêchant à Antioche, comme il descendoit un jour de la chaire, une pauvre femme le tira par l'extrémité de son habit, en lui demandant quand est-ce qu'il prêcheroit pour elle; c'est-à-clire, quand est-ce qu'il se feroit mieux entendre du peuple. Je tiens d'une personne très - versée dans les langues orientales, que les Arabes ont aussi clans leurs écrits les duels, mais qu'ils ne s'en scrvent jamais dans leurs discours, et que, quand elle vouloit les employer en parlant avec les habitans du pays, on ne lui répondoit gư'au pluriel, 
sur l'íle de Syra. Chap. IX. 185

Je crois donc que tout ce qui regarde l'omission de la plus grande partie des temps des anciens, la manière constante d'énoncer nos futurs et nos infinitifs, et l'abandon que nous arons fait de toutes les manières élégrantes de construire !es plrases, ctc. nous viennent de ce langage du peuple, qui, comme nous l'itvons dit, a dû exister dans tous les temps. Én. suite les différences qui serencontrent entre plusieurs de nos mots et ceux des anciens peuvent dériver de ces dialectes inconmus, qui, avec le temps, sur - tout sous les empereurs de Constantinople, lorsque tous les Grees étoient soi:mis à cet empire, auront prévalu, et qui s'étant mêlés avec le langagre commun, se seront éiablis chez les différens peuplés de la Grèce. En effet, si on vouloit faire une recherche exacte; on trouveroit que la langue actuelle des Giecs n'est en partie qu'un méiange presque de tous les dialectes connus; par exemple, nous disons

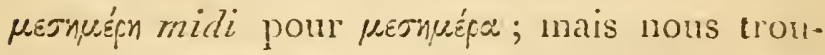
vons que les Ioniens disoient rijuśn pour riéépe. Au lieu de l'accusatif pluriel, nons nuus semones du datif pluriel, et du gérnitit singulier, au lisu du datif singulier; mais cela se pratiquoii anssi chez les Eoliens, yui diecient zxג wis datif pris- 
riel, an lieu de $x a \lambda a . s$ accusatif phriel; ct oopaiss pour copa's.

Il faut répondre à un reproche que l'on nous a fait, d'avoir adopté plusieurs mots turcs et italiens dans notre langue moderue. Cela est vrai, mais il est vrai aussi qu'il est orclinaire aux langues vivantes d'emprunter d'une autrc. Pour ne pas chercher les preuves bien loin, combien de mots nouveanx remprunte pas tons les jours la langue françoise? Ne peut-on pas en dire autant des anciens Grees vis-ḋ-vis les Phéniciens, les Perses et les Romains mêmes, dont cependant la langue est presque la moitié composée de celle des Grecs? Et trouve-t-on extraordinaire que les Grees modernes aient emprunté quelques mots des Italiens, avec lesquels ils ont eu et ils ont ençore autant de liaisons, et des Turcs, anxquels ils sont soumis depuis plusieurs siècles? Ce reproche est d'autant moins juste, qu’indépendamment des mots turcs on italiens qui se sont gुlissés insensiblement dans notre langue, nous en avons d'autres qui expriment la même chose. Par excmple, nous avons pris des Italiens le $\sigma x \tilde{z} \pi \%$ ballet, mais nous avons deux autres mots grees, qui expriment la même chose, ox́pwea ct 
SUR L'île de Syra. Chap. IX. 187 Nous arons des Tures, $\alpha$ 'v'خ ivas ill est d'usage, et $\alpha y \alpha^{\prime}{ }^{\prime}$ liberté; mais nous exprimons ces mêmes

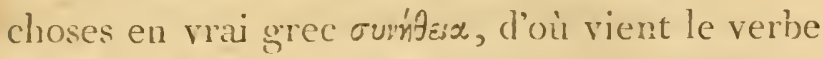

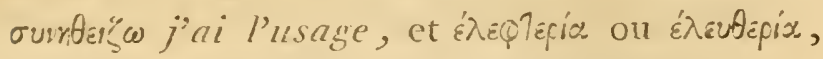

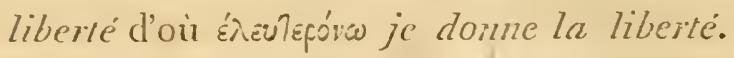

- Une chose que notre langne a adoptée, quil semble que les anciens ne connoissoient pas, et qui, selon tonte apparence, est un barbarisme,

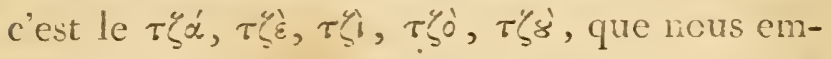
ployons en plusieurs mots, comme $\tau_{s}^{\prime \prime} \alpha: \alpha_{i}^{\prime \prime} \omega$ je

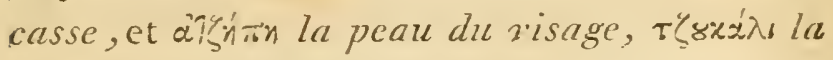
marmite. Cela cependant peut avoir tiré son origine de la difficulté que lespeuples de quelques contrées de la Grèce, ont pu avoir de prononcer quelque lettre. En effet, nous voyons encore aujoard'hui les habitans de quelque île de l'Archipel, qui ne peurent du tout prononcer

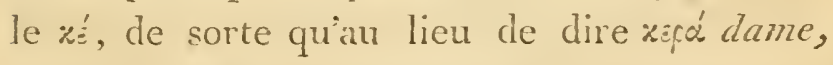

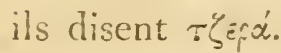

Cuncluons donc, d'après tont ce que nous renons de dire, que la langne que parlent les Syriotes et tous les Grecs modernes, est nne vraie langue grecque, qui nous a été transmise par nos ancêtres, qnoique très-paurre ct moins élégante que colle d'Homcre et d'autres fameux écrivains. Cela est si vrai, qu'un Grec, que 
qu'il soit, s'il s'adonne à l'étude des auteurs anciens, est en état d'y faire plus de progrès en trois mois, qu'un étranger en trois ans.

P.S. Avant de terminer ce chapitre sur la langue grecque, je rapporterai une note de M. l'abbé Barthelemy, sur la formation des différens dialectes de la Grèce, et sur le langage qu'Homere a employé dans la composition de ses ouvrages, qui a du rapport à notre sujet, en y ajoutant quelques réflexions. Voicises paroles, note première, à la fin du premier volume de son excellent ouvrage des Voyages d'Anacharsis.

"Homere emploie souvent les divers dialectes de la Grèce, et on lui en a fait un crime. C'est, dit-on, comme si un de nos écrivains mettoit à contribụtion le Languedocien, le Picard et d'autres idiomes particuliers. Ce reproche paroît bien fondé : mais comment imaginer qu'avec l'esprit le plus facile et le plus fécond, Homère se permettant des licences que n'oseroit prendre le moindre des poètes, cût osé se former pour construire ses vers, me langue bizarre, et capable de révolter non-seulement la postérité, unais son siècle même, quelque jgnorant qu'on le suppose? Il est donc plus naturel de penser 
SUR L'île DE SYr A. Chap. IX. 189 qu'il s'est servi de là langue vulyaire de sorı temps."

"Cliez les anciens peuples de la Grèce, les les mêmes lettres firent d'abord eutendre des sons plus ou moins discordans, plus ou moins ouverts; les mêmes mots eurent plusieurs terminaisons, et se modifierent de plusieurs manières. C'étuient des irrégularités, sans doute, mais assez ordinaires dans l'enfance des langues, et qu'avoient pu maintenir pendant plus longrtemps parmi les Grecs les fréquentes émigrations des peuples. Quand ces peuplades se furent irrévocablement fixées, certaines façons de parler devinrent particulières en certains cantons, et ce fut alors qu'on divisa la langue en des dialectes qui eux-mêmes étoient susceptibles de sous-divisions. Les variations fréquentes que subissent les mots dans les plus anciens monumens de notre langue, nous font présumer que la même chose est arrivée dans la langue grecque. »

* A cette raison générale, il faut en ajouter une qui est relative aux pays ou Homère ćcrivoit. La colonie Ionienne, qui, deux siècles avant ce poète!, alla s'établir sur les côtes de l'Asie mineure, sous la conduite de Nélée, fils 
190 PRÉCIS HISTORIQUE

de Codrus, étoit composée, en grande partie; des Ioniens du Péloponèse; mais il s'y joignit aussi des habitans de Thèbes, de la Fhocide et de quelques autres pays de laGrèce (I). »

"Je pense que de leurs idiômes mêlés entre eux et avec ceux des Éoliens, et des autres colonies grecques voisines de l'Ionie, se forma la langue dont Homère se servit. Mais dans la suite, par les changemens progressifs, qu'éprourverent toutes les langues, quelques dialectes furent circonscrits, et conscrvèrent néammoins des variétés qui attestoient l'ancienne confusion. En effet, Hérodote, postérieur à Homère de 300 ans (2) reconnóît quatre subdivisions dans le dialecte qu'on parloit en Ionie (3). «

Ce que nous avons dit ci-dessus sur l'origine et la formation des différens dialectes de la Grèce, et sur l'usage qu'Homère en a fait dans ses ouvrages, semble contredire l'opinion de M. l'Abbé Buthelemy : en effet, il n'est guère probable que le peuple, dans les temps de ce grand Poète, ni dans d'autres plus rapprochés, parlât nne

(I) Pausanias 1.7, c. 3, p. 5 เ

(2) Id. I. I , c. 1.13

(3) Hérodot. 1. 2 , c. 53. 
SUR L'île de Syra. Chap. IX. IgI langue siriche, si variée et si élégante, que celle d'Homère. Je crois qu'il a toujours existé parmi les Grees un langage vulgaire, qui étoit moins noble que celui des Philosophes et des Orateurs; mais je ne pense pas que celui d'Homère fût de cette dernière classe.

Tous les peuples, dans leur idiôme ordinaire, peuvent se servir de plusicurs synonymes pour énoncer le même objet ; mais généralement ils ne les expriment, et ils ne les écrivent que de la même manièrc.

Nous yoyons ąu contraire que dans les dialectes grees, outre qüil y avoit plusieurs synonymes différens, il y avoit aussi quantité de mots différemment écrits et prononcés.

L'enscmble de tous ces dialectes n'étoit donc pas un langage de quelque peuple en particulier, c'étoit l'idiôme de plusieurs peuplades, qui s'entencioient entre elles, à cause de leur grande communication, par la situation et la disposition des différens pays qui composoient la Grèce; on peut dire qu'il n'en existe pas dans l'univers de plus heureux pour la communication des habitans. La Grèce entière étoit remplie de côtes et d'îles infiniment rapprochées. Les cćrémonies religieuses des Grecs, et leurs institutions 
civiles et politiques rendoient la communication indiepensable: leur étroite union leur facilitoit Vintelligence des différens idiômes qui étoient en usage parmi eux. Cette circonstance a pu readre Homère moins scrupuleux à se déterminer à écrire dans tous les dialectes grecs.

Cela cst si vrai, que Plutarque, dans ses observations sur les dialectes dont Homère a fait usage, nous dit: "Que ce poète s'étant servi "d'une manière de parler très - variée, a mêlé " enscmble tous les caractères des dialectes grees; "d'où l'on doit conclure, dit-î, qu'il a dû pour " cela parcourir toute la Grèce ct toutes les na"tions. " Un écrivain qui lait usagge d'un style bigarré ( $\pi$ ooxi $\lambda x$ ), qui mêle ensemble les difiérens idiômes de plusieurs peuples, qui parcourt enfin toute la Grèce, pour en connoître tous les dialectes, ne peut pas avoir employé la langne vulgaire de son temps.

Qnoi quil en soit, celui qui mêleroit le jargon lanģuedocien à d'autres jargons, mériteroit heancoup de ridicule. Plutarque a confirmé 'u'Homere s'est effectivement servi dans ses ćcrits diun langage très-bigarré. Il assure encore "que ce poète, de même qu'il a ramassé les ex"pressions de tous les Grees, en formant unc 
sur l'île de Syra. Chap. IX. 193 * manière variée de parler; de même il se sert * quelquefois des expressions étrangères, tantôt * des anciennes, et tantôt des communes, et * de celles qui étoient en usage. C'est une chose, " conclut Plutarque, digne d'admiration, que * dans Homère, les expressions les plús vulgaires " conservent une élégante gravité. „Malgré cela, loin que ses ouvrages, par cette variété, aien jamais révolté personne, ils ont fait au contraire, dans tous les temps, l'admiration et les délices de tous les connoisseurs et des gens de goût.

Si l'on me demande pour quelle raison $\mathrm{Ho}_{\mathrm{O}}$ mère a écrit ainsi, je répondrai, que pour un génie aussi vaste que le sien, pour une imagination aussi féconde, il falloit, pour suivre la rapidité de ses pensées, des expressions abondantes et hardies: il les prenoit dans différens dialectes, quand elles saccordoient à la mesure de ses vers. Son enthousiasme ne pouvoit souffrir aucun retard; et quand il lui falloit de nouveaux moyens pour s'exprimer, il employoit tous les clialectes usités parmi les Grecs.

Sans vouloir attaquer le systême de M. l'Abbé Barthelemy, sur l'origine et la formation des différens clialectes de la Grèce, je dirai seulement en consıdérant la quantité et la variété de ceux

Tome $I$. 
I94 PRÉCIS HISTORIQUE qui existoient chez les Grecs, et dont on ne voit d'exemple chez aucun autre peuple, qu'ils tenoient à leur caractère et à leur génie. Vifs et ardens à saisir les objets, portés plutôt à l'invention qu'à l'imitation, ils changeoient ou ajoutoient à tout ce qu'ils avoient appris : leur mythologie en est une preuve sans réplique. 


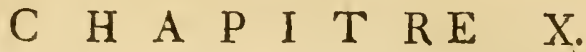

De quelyues procédés particuliers relatifs is l'agriculure et à l'économie domestique.

I. Toutes'les terres labourables de notre île sont divisées en deux parties, que l'on ensemence alternativement, de manière que tardis que l'une est en rapport, on laisse reposer l'autre. Celle-ci ne reste pas tout-à-fait inutile, mais on n'y sème que des légumes et des plantes qui tirent peu de nourriture. Cette partie non semće, s'appelle alors paslra, et l'autre, engheria. $2^{\circ}$. A Syra et dans tout l'Archipel, on se sert de l'araire pour labourer la terre. Le terrain de ces îles étant en général très-fort, quelquefois peu profond, souvent pierreux et raboteux, il seroit impossible de labourer avec la charrue: ce sont toujours des bouf's que l'on y attèle, et jamais d'autres animaux.

Voici ce que dit le Journal de Paris, $n^{\circ} .350$, sur la différence de la charrue et de l'araire. 
196 PRÉCIS HISTOR I Q U E

« L'araire est l'instrument des montagnes et * des bois, et la charrie est l'instrnment des « plaines et des pays ras. L'araire peut toujours « remplacer la charrue, et la charrue ne peut « pas toujours remplacer l'araire. La principale «différence entre ces deux instrumens, c'est "que l'un a des roues, et l'autre n'en a pas. «L'araire est de toute antiquité; la charrue ne " date que de dix-sept siècles. Le P. Monfaucon " s'est trompé, quand il a dit que les anciens ne " connoissoient pas la charrue; elle est anté« rieure à Pline, mais je la crois postérieure à «Virgile."

$3^{\circ}$. Pour disposer une terreà recevoir du bled ou de l'orge, on lui fait subir à-peu-près la même préparation qu'aux jachères en France. C'est cette préparation que l'on appelle pastra, melrcıé, et qui a fait donner le même nom à une terre en cel état: elle consiste à lui donner trois labours; le premier, vers la fin de février; le second, dans le mois de mars, et le dernier, vers le milieu d'avril; de sorte qu'on laisse une vingtaine de jours environ d'iutervalle entre chacun. Dans les deux demiers, on a soin de tirer les sillons, de manière qu'ils compent transversalement ou obliquement ceux des labours pré- 
S UR L'île de Syra. Chap. X. 197 cédens. Les labours faits, on sème les légumes d'été et le coton. On jette encore entre autres quelques graines de sézame, de melons et de melońs d'ẹau, qui y viennent très-bien, quoiquil ne pleure pas quelquefois de cinq mois. Dès que ces plantes ont commencé à pousser, on sarcle la terre, et on la nettoie de toutes les mauvaises herbes : ce travail se répète souvent jusqu'à trois fois avant la maturité des légumes et des cotons. L'automne étant venue, l'on sème le bled ou l'orge. Il est aisé de concevoir qu'avec de tels soins, on doit se procurer cles grains et des légrumes de la première qualité; aussi préfère-t-on communément ceux de Syra à ceux des autres îles.

$4^{\circ}$. Avant de semer leur coton, les Syriotes donnent une préparation à la graine. On sait que celle du cotonnier, après avoir été séparée de la bourre par le moulinet, conserve toujours une espéce de duvet, qui la tient liée par paquets, et la rend difficile à semer. Pour remédier à cet inconvénient, on la mêle avec du sable des torrens; on verse de l'eau par dessus; on la remue bien, en la frottant avec les mains sur une pierre plate, jusqu'à ce que tout le duvet soit détaché; ensuite on la relave pour la dé- 
barrasser du sable, et on la sème alors avec facilité.

Le coton se sème très-clair. Quand il a acquis une certaine hauteur, on l'étête pour lui faire pousser une grande quantité de rameaux, et plus de fruits. Il arrive de là que nos cotonniers s'élèvent rarement à plus d'un pied: ils demandent un terrain sec; ceux qui sont dans des terrains bien humides,s'élevent trop, et ne produisent que peu de coques: c'est pour cette raison que les années pluvieuses leur sont contraires... Quoique les coques des cotonniers de Syra ne soient pas de la grosse espèce, le coton en est cependant d'une très-bonne qualité ; il est un peu rongeâtre, comme le terrain ; mais lés toiles que l'on en fait acquierent, après quelques lessives un assez beau degré de blanchemr.

$5^{\circ}$. Les insulaires de l'Archipel ont plusieurs manières de conserver leurs graines : ils font dans les champs un trou proportionné à la quan. tité de bled qu'ils y veulent serrer ; il est ordinairement de cinq pieds de diamètre, sur deux ou trois de profondeur. On en tapisse l'intérieur d'environ un demi-pied de paille brisée sous les pieds des boufs; on y serre ensuite le brain, de manière quill s'élève par dessus la 
sur l'île de Syra. Chap. X.

terre, à une hauteur à-peu-près égale à la profondeur du trou; on le convre avec un demipied de paille, sur laquelle on met trois ou quatre pouces de terre. A Syrà, on a renoncé à cette pratique, à cause de plusieur's inconvéniens. Aux premières pluies d'octobre ou de novembre, on étoit ohligé de retirer le grain de la fosse, que l'humidité auroit fait gâter. On préfere actuellement de le mettre dans des granges, où l'on conserve en même temps tonte la paille pour les bestiaux. Ces grranges, appelées en grec $\theta$ cesoréx, ont communément une vingtaine de pieds de long, sur huit à dix de hauteur et de largeur. On les remplit jusqu'à la moitié de'leur hauteur, de paille bien fuulée: on pratique un espace de trois ou quatre pieds, que l'on remplit de grain. A cûté on en forme un autre, que l'on remplit de même, et ainsi de suite, selon l'étendue de la grange, et la quan. tité de grain que l'on a cela fait, par des ouvertures pratiquées dans la couverture, on recouvre de paille tout le bled, jusqu'à ce que la grange soit exactement remplie. Quand on veut en faire usage, on commence par le tas le plus voisin de la porte; on enlève d'abord la paille avec beaucoup de précaution : plus on ap- 
proche, plus cette précuution augmente; enfin, pour ôter les derniers brins de paille, on se sert d'un balai de millepertuis, ou d'autres plantes que l'on fait sécher; et si malgré tous ces soins, la surface du mouceau de grạin n'est pas bien nette, on achève d'en enlever toutes les menues pailles en la vannant avec un chapeau, car les paysans de nos îles portent, comme ici, dans les champs, des chapeaux ronds de feutre; ils en portent aussi de paille, que l'on travaille avec beaucoup de délicatesse à Sifanto. ¿Ceux enfin qui veulent porter leurs grains à la ville, les mettent dans des vases de terre cuite, qu'ils remplissent à deux ou trois pouces près; ensuite ils étendent par dessus quelques feuillesde figuier sauvage, appeléorni, et en latin, caprificus; enfin ils achèvent de remplir les vases avec de la cendre, et les couvrent d'une espèce d'ardoise, mais plus forte et plus épaisse que celle dont on se sert en France pour couvrir les maisons.

Quelle que soit au reste la méthode que l'on emploie pour conserver le grain, il n'y a point à craindre quill soit endommagé par les insectes, puisque le défaut d'air empêche d'éclore les œufs qui pourroient y avoir été déposés. 
SUR L'îlE DE SYra. CHAP. X. 20I

$6^{\circ}$. Les Syriotes et les autres habitans des iles de l'Archipel, ont aussi une manière particulière de couvrir leurs habitations. Les maisons sont en pierre, liées avec un mortier de terrevierge; elles nont jamais qu'un étage, ảiózı

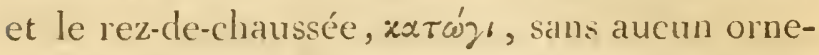
ment extérieur; on les blanchit seulement quelquefois avec de la chanx, ou on les recrépit, du côté du nord, arec un très-bon ciment, composé de chaux et de sable des torrens. Quand les murs sont élevés, on met les solives, ou si l'édifice est trop large, on place une poutre à sept ou huit pieds de distance du mur, ou bien on construit mne arcade en pierre au lieu de la poutre. Sur ces solives, qui sont à la distance d'environ un pied les unes des autres, on pose de la manière suivante de gros roseaux, qui croissent en abondance clans l'Archipel. D'abord entre chaque solive, on place un roseau des plus gros, dont on assujettit l'un desbouts sur le mur, et l'autre sur la poutre, cnsuite l'on met par dessus un rang d'autres roseaux, qui croisent les premiers, et auxquels on les attache avec une corde de jonc ; après quoi on étend un lit d'une espèce de joncs appelés en grec vurla, sur lesquels on met une 
grande quantité d'algnes, que l'on ramasse a au bord de la mer. Après avoir bien foulé les uns et les autres, de manière qu'ils ne fassent qu'un même corps de 5 à 6 pouces d'épaisseur , on recouvre le tout de terre vierge, ou d'une terre tiréc des décombres pulvérisées. Cette terre, bien foulce avec $m$ rouleau de marbre, achève de remplir un vide de so à 12 pouces d'élévation, qu'on donne au mur au dessus des solives lors de la construction, et qu'on destine a contenir les roseaux, les jones, les algues et la terre. En bâtissant, on a la précaution de laisser sur un des quarre murs, un ou plusieurs trons pour servir de grouttières. On les place ordinairement du côté du midi, à cause des vents du nord: on a soin de diriger l'écoulement de toutes les eaux par ces gontières, en domant une pente à toute la terrasse, vers ce même côté-

Tels sont les moyens simples et peu dispendieux qu'on emploie dans notre ile pour construire des maisons qui durent quelquefois jusqu'à cent ans, sur-tout si l'on a soin de crépir les murs en dehor's, à chaux et à sable. Il n’y entre ni fer, ni autre métal. La toiture est preśque aussi durable, et n'exige guère d'aute vi 
SUR L'íle de SyRa. Chap.X. 203 entretien, que de détruire l'herbe et la mousse qui peuvent y croître, de renouveler de temps en temps la terre des bords, qui est plus sujette à se dégrader, et d'y passer le rouleau de marbre, pour comprimer et raffermir la terrasse.

$7^{\circ}$ J'ai dit qu'à Syra, on donnoit le plus grand soin à la culture de la vigne. On plante les ceps à trois ou quatre pieds les uns des autres; on les tient couchés sur la terre, à cause des vents du nord qui soufflent une bonne partie de l'été, et qui les casseroient sans cette précaution; mais on laisse les nouvelles branches s'étendre tant qu'elles rculent, de sorte que la terre en est presque entièrement couverte. Il n’y a point à craindre que les grappes se pourrissent; au contraire, la chaleur de la terre ajoutant à l'action du soleil, contribue à accélérer la maturité. Au reste, dans les plaines, et les vallons, où la terre est plus humide, ct où le raisin pourroit se gâter, on soutient le sarment tout près de la grappe, avec de petites fourcbes de bois, que l'on enfonce dans la terre; mais presque toutes nos vignes sont situées sur le penchant des montasnes, ou dans des lieux secs. Il y a deux espéces de raisins dont on fait du vin, le noir et le blanc; 
mais on en cultivé pour la table, d’autres qui sont d'une très-bonne qualité. Le raisin noir est très-serré. On coupe la vigne qui le produit, au dessus du quatrième ou du cinquième bourgeon, tandis que ce n'est qu'au dessus du dixième, et quelquefois même du quinzième, qu'on taille les autres : une longue expérience a appris que ces différentes espèces de vignes vouloient être taillées différemment, pour se conserver plus long-temps, et produire plus de fruits.

$8^{\circ}$. A Syra on laboure les vignes avec l'araire, et on $y$ est persuadé que cette manière est la plus utile; en effet l'araire, en détruisant les racines les plus profondes des mauvaises herbes, détruit aussi celles des ceps qui sont à la superficie de la terre, et les oblige d'en pousser de plus profondes, qui tirent plus de suc de la terre, et sont moins exposées à souffrir des ardeurs du soleil. Le labour de la houe, au contraire ( car de toutes les îles de l'Archipel, je ne connois que Chio, où l'on se serve de la bêche), la houe, dis-je, ne détruisant pas ces racincs superficielles avec la même efficacité , elles s'étendent et se multiplient extraordinairement, ce qui nuit beaucoup 
SUR L'île de Syra. Cha P. X. 205 à la tige. On objectera que les boufs, en passant dans les vignes, doivent briser des branches qui auroient pu donner du raisin; mais nos vignerons regardent cette perte comme amplement compensée par la vigueur que doit procurer à la tige une terre mieux labourée, et par la qualité des grappes et la grosseur des raisins qu'elle doit produire. D'aillcurs, pour éviter trop de dégâts, il y a toujours quelqu'un qui marche devant les boufs, pour relever lesbranches. On supplée avec la houe, à ce qui n'a pas été labouré au tour des tiges par l'araire.

$9^{\circ}$. Le provignement est une partie de l'agriculture, qui avoit été bien négligée à Syra, et qu'on a remise en vigueur. Voici la méthode que j’ai suivie. Pour remplir une vigne, qui étoit à moitié dégaruie, la première année j’ai arrêté tous les ceps; c'est-à-dire, que je leur ai ôté beaucoup de bois, en ne leur laissant que quelques branches. Ces ceps, ainsi arrêtés, ont prisclela force, et m'ont donné presque tous, dès l'année suivante, des branches bonnes à être provignées. S’il y en avoit de trop courtes pour parvenir à l'cndroit où je les voulois conduire, je les provignois dans la longueur qu'elles pouvoisnt s'étendre, pour les reprovigner plus 
loin la seconde année; ou je les alongeois en les greffint avec d'autres branches. Le dernier moyen, que j'ai appris d'un de mes parens, qui a été $2+$ ans Vicaire Apostolique à Sifanto, ma été d'une très - grande utilité pour regarnir plustôt mes vignes, pour multiplier quelques espèces de raisins qui y manquoient, pour rendre mes vignes plus fertiles, parce que pour greffer sur les provins trop courts, je choisissois des branchesdes tiges les plus fécondes et de la meilleure espèce.

Avant d'aller plus loin, je vais rendre compte de ma manière de grefier la vigne dans cette circonstance. Je coupe immédiatement à côtéd’un nœud, chacune des tlenx branches que je veux greffer, de manière que ce nocud soit abattu. Je fends l'une de ces deux branches jusqu'à l'autre nœud, c'est-à-dire, en forme de rainure de deux ou trois ponces de long; j'amenuise l'autre branche jusqu'à la moelle, en forme de languette, d'une pareille longireur, et je l'insère dans la prenière; je les serre étroitement avec du jonc, et je les couvre de terre.

Dès la deuxième année, chacun des provins jette trois on quatre ponsses, capables d'être ellesmêmes provignées; je les enfonce toutes, je pro- 
Sur l'fle de Syra. Chap. X.

gne les plus longues, et je redressela plus courte pour occuper la même place. Tel est le moyen que j’ai employé, et qui m’a si bien réussi, qu'en trois ou quatre ans, une petite vigne s'est trouvée regarnie, et que d'un fagot de sarment,et d'une outre de vin qu'elle rendoit par année, elle ne tarda pas à m'en donner douze à quatorze, de vin blanc de qualitésupérieure, et dix à douze fagots de sarment. Au reste, ce même moyen m'a servi encore à renouveler peu-à-peu et entièrement mes vignes, parce que j'avois l'attention, au bout de trois ou quatre ans, de détacher les provins de la vieille souche, et d'arracher celle-ci. Si cependant cette souche étoit encore saine et vigoureuse, je la laissois subsister; $j$ 'en détachois seulement les provins en coupant ceux-ci d'abord à l'enclroit où ils tenoient immédiatement à la tige-hère, puis à un pied environ au dessous au pli qu'on leur avoit fait pour les prolonger sous terre. Cette manière de provigner et de renouveler les vignes, est prélérable à celle qu'on employoit autrefois à Syra, et qu'on emploie encore à Naxie, qui consiste à enterrer tout-à-fäit chaque année les plus, vieilles tiges, et à provigner leurs branches. Il arrive de là que la vieille souche, 
208

\section{PRÉCIS HISTORIQUE}

torjours malade, danguissante et pourrie, ou criblée des insertes qui y déjosent leurs œufs, communiqne aux provins les vices qu'elle a contractés, et qui les minent insensiblement. Au contraire, datis ma méthode de provigner de jeunes branches, en séparant, après quelques années, mes provins, je les ai toujours vus, sains et vigourenx, acquérir de plus en plus des forces nouvelles.

Pour multiplier la vigne de treille, j’ai imaginé un autre procédé. Quand je voyois quelques parties d'une treille on d'un berceau découvertes, je prenois un jet de l'année, le plus éloigné du maître brin, et le plus voisin de l'endroit dégarni; je le recombois, et jen rabaissois l'extrémité, que je plantois en la couchant dans la terre à la profondeur d'un pied et demi. Quand le jet de l'année n'étoit pas assez long pour venir à mon but, je pliois un peu le ivieux bois ; ensuite j'abattois tous les bourgeons, à un seul près, que je destinois à fournir la principale branche qui devoit recouvrir la place vide. Il né faut pas confondre cette manière de muliplier une vigne, avec l'usage où l'on est de les marcotter. La marcotte n'est qu'une branche que l'on replonge en terre pour lui 


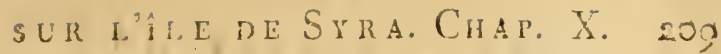
faire prendie racine, et dont le bont sort de la terre, de manive qrielle croit toujours dans son sens naturel, au lieu que snivant ma méthode, quand l'extrémité de la branche replongée entièrement dans la tcire a pris racine, la seve remonte dans cette branche, contre son sens ordinatre. J'ai répété plusicurs fois cetie opération à Versailles, dans le jardin de $M$. Ruffin, Secrétaire, Intepprete da Iioi, pour les langues orientales, où elle a parfaitement renssi. Aussi existe-t-il une tradition lans le Lerant, que le fameux Platane de lile de Cos dans l'Archipel, qui cstsoutenu par dix-liuit colomes de marbre tellement encastrées dans ses bianches énormes, que quand il cst agité par le vent, il sculève ces colonnes; il existe, dis-je une tradition que ce p'atane assez touffi, pour courrir de son ombre me grande giantité de boutiques, a été planté à conire-sens, cent-ì-tive, les branches en tcre, et les raciucs en halut. Peut-être y a-t-il cu là criginarement un platane, dont quelque branche rabattue vers la terre, a repris racine, et à été détachée dü trome qui a péri.

10". Nos procédés pon faire le rin sont bien diférens re coux grion empluie en France I'onle $I$. 
Nous n'avons pas de pressoir, mais seulement des espèces de cuves, que nous appelons patiliri, c'est-à-dire fouloir : chaque vigne a le sien. Ce sont des aryges de pierre carrées, plus ou moins grandes, bâties hiors de terre, et bien cimentées en dehors ct en dedans : en les construisant, on a soin de leur donner de la pente, pour faciliter l'éroulcment de la liqueur, vers une décharge pratiquée à l'un des côtés, et qui répond à un petié bassin construit immédiatement auprès clu grand, mais enfoncé dans la terre et destiné à recevoir levin. Lorsque le temps de la vendange est venu, et que le raisin est bien mûr, on le coupe, et on le laisse exposé au soleil pendant cinq à six jours: on le porte ensuite dans le fouloir, où on le laisse encore reposer cinq à six jours ou même davantage, júsqu'd̀ ce qu'il ait bien fermenté, ce qui fac!ite la séparation de la liqueur. Alors on le foule avec les pieds, puis on le jette peu-ì-peu, dans une claie qui s'ouvre plus ou moins à volonté, et semblable, à peu de chose près, à ces paniers, où l'on tient rassemblés dans les marchés, les jeunes pigeons et les canards. Ces claies sont faites de laurierrose, qui croît abondamment dans les vallons de l'ile. A mesure que l'on y met 
SUR L'île DE Syr A. Chap. X. 2 II le raisin, un homme monté cledans, le fonle et on continue ainsi tant que la claie en peut contenir. Quand elle est pleine, s'il y a cncore du raisin à presser, on défait la claie, on la retire, et on la place dans une autre partie du fouloir, en répétant la même opération trois et quatre fois, s’il est nécessaire, selon la quantité de raisin : quelquefois même on se sert de plusieurs claies à la fisis, et on augmente à proportion le nombre des ouvriers.

Après la pression et la claie enlevée, le marc reste en pains plus ou moins épais, et d'un diamètre plus ou moins considérahle. On lesécrase, on les éparpille clans le fouloir; on verse par dessus une certaine quantité d'eau; cंest ordinairement une ou deux mesures sur dix de vin, que l'on a pressurées. Sans cette précaution, il seroit impossible d'extraire tuate la liqueur, qui a été presque calcinée dans les graius; et il y a des quartiers où les terres sont si fortes, et le vin si violent, que si l'on ne mettoit de l'eau et du moûten quantité égale, levin ne manqueroit pas de s'épaissir et de s'aigrir. On recommence à fouler, cn arrosant à plusieurs reprises, le marc arec du vin du petit bassin, puis on emploie une seconde fois la claie; on 
212 PRÉCISHISTORIQUE

entortille les pains de raisin; ils en sortent, chacun avec un ou plusieurs brins de sarment garnis de leurs petites branches et de leurs feuilles. Ce sont toujours des pousses de l'année, que l'on prend, et quelques-unes ont plus de quinze pieds de long; si une seule ne suffit pas, on l'allonge avec une seconde et une troisième que l'on y noue. On fait entrer un des bouts de ces liens dans le bas du pain, autumr cluquel on lui fait faire plusicurs tours en le serrant bien. On ramène l'autre sur le sommet, de ce même pain où on le fait aussi entrer, et on met par dessus de grosses pierres, dont le poids achève d'exprimer la liqueur. On lave l'intérieur dı fouloir et tous les instrumens qui ont servi ¿̀ l'opération, avec de l'eau qui retombe dans le petit bassin, où elle se mêle avec le vin, ou bien on la met dans le vase où le vin a déja été retiré, car rarement le petit bassin peut-il contenir tout celui qu'on a pressé.

Quand le marc ne donne plus de liqueur, on le jette dans le petit bassin; on le foule encore, on le charge arec de grosses picrres; on le laisse bouillir pendant quelques jours, puis on remplit les interstices des pierres avec 
SUR L'îLE DE SYRA. CHAP. X. 2:3 des feuilles de vigne; on couvre le tout avec de la terre; on le foule bien, et on le laisse dans cet état jusqu'à ce qu'on le retire pour en faire de l'eau de vie. C'est ordinairement vers le mois d'octobre, le matin à la roséc, que les paysans récoltent le coton; et dans la journée en l'épluchant, ils soignent leur alembic. Il y en a qui, au lieu de laisser leur mare de raisin dans le petit bassin, le mettcut dans de grands pots de terre cuite; et quand ils reulent hàter la fermentation, et faire tout de suite leur eau de vie, ils le détrempent avec de l'eau: sinon, quand le mare bien foulé a bouilli, ils ferment hermétiquement avec de la terre franche le couvercle des pots, et conservent ainsi ce marc pendant quatre et cinq mois. L'eau devic qu'on en retire est excellente et d'un goût parfait, sur-tout si on la fabrique avec soin, et si en la distillant, on y jette quelques graines d'anisou de fenouil. Ce goût de fenonil, au reste, ne pent manquer dese communiquerplus ou moinsà l'eau de vie; parce que pour empêchei le mare de s'attacher au fonds de l'alembic, on a soin de le couvrir de quelques branches de fenouil qui le soutiennent: je n'aj pas besoin d'ajouter, ‘ce scmble, qu'on le détrempe premie- 
ment avec muitić d'earu. Ce qui reste au fond de l'alembic aprés la distillation, n'est point enrore inutile, on en sépare les pepins dont on nourrit les pigeons et les porcs. Nous croyons qu'on ne pourroit pas tirer le même parti de ces pepins sils n'avoient point passé à l'alembic; ils fournirojeut sans cela un aliment trop âpre.

$11^{\circ}$. Nous n’avons pas dans nos îles les mêmes moyens qu'en France, poum transporter le rin et pour le conserver : nous le voiturons dans dés outres failes de peaux de boucs ou de petits veaux, que nons savons prépares de manière à ne domner aucun goût à la licquemr Nuus le conservons dans des vases de terre non vernis, mais enduits arec de la cire, et nous le déposons, non dans des caves souterrines, la plupart des maisons étant bâties sur des rochers, où il seroittrès difficile d'en creuser, mais dans des celliers bâtis au rez-de-chaussće.

Cet enduit s'applique de deux manières, ou en frottant avec un morceau de rire l'intériem du vase, immédiatement aprés qu’il est sorti du four du potier, on en le faisant chantfer avant, et après l'avoir frotté, afin que la cire simbibe bien dans tous les pores. Cet enduii dure aussi long-temps que le vase; 
SUR L'îLE DE SYRA. ChAP. X. 215 il résiste à toutes les liqueurs, soit à l'eau de vie, au vin, au vinaigre, à l'huile, etc. il empêche le vin de prendre un maurais goût, et en prévient l'évaporation.

M. Duhamel a propusé de se servir rle citernes, ponr conserver le vin au défaut de vaisseaux de bois, qui, dans certaines années, et dans plusieurs provinces, sont très-difficiles à se procurer; mais il se plaint, et du mauvais goût que le mortier donne au vin, et de la déperdition qui se fait par les pores de la citerne. Je suis persuadé que le moyen que je viens d"indiquer, remédieroit à ces inconvéniens. Pow cela on pourroit allumer dans la citerrie un feu capable de l'échanffer en entier: dès qu'elle auroit acquis le degré de chaleur désiré, plusieurs personnes y descendroient à-la-fois, et se meitroient à en frotter les parois avec de la cire. Si, après cette opération, il restoit quelque place qu'on eût oubliée, ou qui se fût refroidie, on pourroit la chauffer à l'aide d'une plaque de fér d'un pouce d'épaisseur, sur un pied en carré, que l'on auroit fait rougir au feu. On pourroit aussi se servir à peu près du même noyen pour communiquer une chaleur moindre aux parties on la cire ne se seroit pas assez. 
imbibée; par exemple, en fasant moins chauf. fer la plaque, ou cu ne l'appliquant pas immédiatenent an mur. Au reste, despersonnes intelligentes et ćlairées, pourront profiter des idées que je n'ai qu'ébauchées, et imaginer les moyens les plus propres pour exécuter cette opération. On mobjectera qu'en échauffant la citerne arec de la braise, ou de quelque antre matiere, il y a à craindre que la vapeur n’incommode cewx qui descendroient pour l'enduire; nais ne peut-on pas remédier à cet inconvénient par une foule de moyens anti-méphitiques, qui cont assez connus?

$12^{\circ}$. Nousavons aussi à Syma des pratiques trescirbuses et trés-utiles sur lincubation des poules. Nous commencons par examiner, avec la plus grande attention, tous les cufs que nous destinons à être couvés. Ceux dans lesquels nous inercevons de petits flobules clairs et faits en formo d'ćtoiles, sont tonjours stériles. Parmi cewk qui restent, nous examinons quels sont aux qui doivent donner des cochets, et cewx qui juroduiront des poulettes: on les distingue à la position de la comronne, ou calote intérieure, que l'on apperçoit au gros bout, Si cette conromo est placée presciue horicontalement, il 
naîtra un coq; si au contraire elle est placéc obliquement, ce sera une poule: on est ainsi le maître de se procurer le nombre précis de poussins que l'on veut daus l'un ou dans l'autre sexe : aussi voit-on rarement chez nous plus de deux ou trois mâles, sur une couvée de quinze à vingt, tandis qu'en France, le nombre des mâles est au moins égal à celui cles femelles (I). Cinq à six jours après que nous avons mis les œufs sous la poule, nous les mirons au soleil, et ceux dans lesquels nous n'apercevons pas des filets de sang, nous les retirons : ceux-là

(1) Voici une particularité qui regarde l'objet que nous traitons, et que je trouve dans une lettre de Madame Vicat, qu'elle écrit au sujet de la fameuse question sur l'histoire naturelle de la mère-abeille. Cette lettre est rapportée par M. Blaissière, dans sa traduction de l'ouvrage de Schirach sur les Abeilles." On assure, dit-elle, qu'on peut connoître par la forme c'un cuf le poulet qu'il renferme, s'il est poulelte, ou cochet, et par la forme d'un cocon, celui d'où doie sortir un papillon mâle ou femelle." Tout le monde connoit cetie particularité, à légard des papillons des vers à soie, mais celle de connôtre le sexe du poulet par la forme d'un ceuf, nous l'ignorons dans le Ierant. 
ne sont point fécondés. Nous trouvons un double avantage dans cette pratique; d'abord ce sont toujours autant d'œufs auxquels la poule est dispensée de donner ses soins; en second lieu, ces œufs sont, à la rigueur encore bons à manger : le moindre usage qu'on en fasse, c'est de les conserver pour servir de première nourriture aux petits qui doivent éclore. Voici encore une attention que nous avons, et qu'on néglige chez les peuples occidentaux. Les poussins, en naissant, ont sur le bout du bec une corne très-dure, que la nature prévoyante leur a donnée pour casser la coque de l'weuf; mais cette corne devenue inutile dès qu'ils en sont dehors, les gêne pour ramasser le grain, spécialement sur les planches, la pierre, et tous les corps durs. Il est donc de l'interêt 'du propriétaire, quand cette corne ne tombe pas d'ellemême, dès le premier jour, de la délacher avec l'ongle, et c'est ce que nous faisons à Syra.

130. J'ai eu occasion de faire, soit dans notre île, soit en France, quelques observations sur les pigeons. J'ai remarqué que chez nous, les deux œufs que produit chaque ménage, sont toujours de sexe différent, tandis qu'en France 
Sur l'ile de Syra. Chat. X. 219 ils sont souvent de miême sexe. J'ai rmarqué encore que c'est toujours le premier pondu qui porte un germe màle. Or, il arrire liéquemment, et sur-tout daus les temps froids, que l'un des deux périt dans la coqne, c'est presque tonjours le male. J'en ai, ie pense, trouvé la raison : les pigeons mettent deux jours d'intervalle entre la ponte de ces deux oeufs; pendant ce temps-là, wi le père, ni la mère ne couvent l'œuf : ils se tiennent seulement de bout sur leur nid, comme pour le garder; mais il arrive quelquefois, et sur-tout pendant la nuit. que l'un ou l'autre se trouvant fatigué, et la mère sur-tout, se repose sur l'ouf, et quela chaleur de l'incubation commence le développement du gaerme; mais bientôt ils se relèvent et le laissent refroidir. Alors le déreloppement s'arrête pour toujours, et l'œuf se gâte; c'est done le mâle qui doit périr le plus souvent, et le moyen de s'assurer que c'est pour cette raison, et non pas parce que l'œư n'a pas été fécondé, qu'il a manqué d'éclore, c'est d'examiner s'il est corrompu ou non dès le dixième jour.

C'est une preuve que le commencement de développement a eu lieu, siuon il reste clair, 
presque jusqu'à la fin. Il arrive souvent la même chose aux œufs de sérins. Aussi y a-t-il des personnes qui retirentles premiers, et qui ne les remettent que quand tout est pondu, et rarement il leur arrive d'avoir moins de petits que d'œufs.

L'auteur de la Maison Rustique prétend que les pigeons ne s'accouplent qu'à l'àge de six mois. J'en ai vu s'accoupler dès le commencement du quatrième mois; à la vérité, ils étoient d’une très-bonne espèce.

Les jeunes pigeons qni muent, tardent à s'accoupler, au lieu que ceux qui ne muent pas, saccouplent plutôt.

Dans le Levant, jai remarqué que les deux pigeons d'uie même couvée s'accouplent toujours ensemble : ici je vois que la chose est assez rare, et que les deux époux se choisissent le plus communément dans deux familles diffórentes. Quand on a de belles espèces de pigeons, et qu'on veut éviter quellés ne s'abâtardissent, on doit avoir une petite volière pour les enfermer dans le temps des amours, qui est toujours indiqué jar les roucoulemens du mâle, et ses empressenens auprès de la femelle. Il est encore esscntiel d'aroir un endroit particulier 
SUR L'île dE SYRA. ChA X. 22 . pour y renfermer ceux qui veulent s'accoupler, parce que si on les laisse en liberté, leur union se fait bien plus tard, tandis qu'elle a lieu dès le premier jour, ou tout au plus au troisième, quand on les resserre; mais il ne faut pas les tenir dans cette gêne pendant quinze jours, comme le dit l'auteur de la Maison Rustique; ils auroient déja fait leur ponte. J'ai eu à Versailles un pigeon mâle, d'une très-belle espèce, à bec court, huppé, les yeux entourés d'un rouge vif: je l'ai marié avec une femelle blanche ordinaire; il m’a donné des pigeonneaux huppés, fort beaux, mais qui n'étoient pas de l'espèce du père. Accouplé arec un de ses enfans, il a produit des petits qui lui ressembloient dayantage; enfin, avec un de ces derniers il a donné une troisième génération qui lui ressembloit parfaitement.

Avant de finir cet article, iy ajouterai quelques remarques que j'ai faites en France sur les pigeons. J'ai observé que dans certains tems de l'année, les mâles suivoient continucllement les femelles. J'ai reconnu que cééoit ordinaiment clans le temps où la femelle étoit prếte à pondre son œuf. Cette poursuite du mâle se fait avec tant d'acharnement, qu“il ne donse 
pas le temps à sa compagne de manger ou de boire ; il la becquete continuellement, jusquà ce qu'il l'ait obligée à rentrer dans leur nid; alors le mále transporte des brins de paille, ou des branches le quelques plantes, pour en former et construire $m$ autre: on scroit tenté de croire que la nature auroit donné cet instinct aux mâles, dans la crainte que les femelles ne choisissent pas un endroit favorable pour pondre leurs œuls. J'ai vu plusieurs fois des femelles, dont les mâles, par maladie ou quelque autre canse, ne les poursuivoient pas, laisser tomber leurs oufs hor's de leur nid.

Ceux qui veuleut donner à ces animaux tous les soins possibles, doivent tâcher de reconnoître les petits, qui tombent souvent de leurs nids: alors il faut connoître leurs mères pous les leur rendre; sans cela ils ne manqueroient pas de périr, si on les mettoit dans un antre.

$14^{\circ}$. Nous avons dans. l'Archipel une manière particulière de chasser la perdrix: le chasseur prend un morceau de toile de diverses couleurs, dont il fait une espèce de bannière, d'environ trois pieds de large sur six de haut. Il pratique une fente au milien pour lui servir de visière et pour passer le bout du fusil. Muni de ces 
SUR L'île DE SYRA. CHAP. X. 223 instrumens, il part de bon matin, et dès qu'il entend chanter la perdrix, il arme son fusil, l'ajuste à sa bannière qu'il porte de la main gauche; et en se tenant toujours exactement caché derrière, il s'avance lentement du côté du gibier. Lesperdrix frappées et étonnées des différentes nuances de couleurs qu'elles aperçoivent sur la bannière, se rassemblent sur une pierre ou sur un mur, elles caquètent, et semblent s'interroger sur ce qu'elles voient : cependant le chasseur' arrive, s'arrête, tire son coup, et communément en jett’̀ à bas huit, dix et davantage. Cette manière de chasser la perdrix est sur-tout très-avantageuse, lorsqu'une mère conduit à sa suite une compagnie de perdreaux, ou quand en hiver ces animaux s'attroupent. Puisque nous en sommes sur l'article des perdrix, je remarquerai qu'à Syra on en roit quelquefois de blanches; ce qui est extrêmement rare, puisqu'en effet on n'en trouve de cette couleur que dans le nord de l'Europe, dans le Groenland, et dans les montagnes glacées des Alpes. Les Turcs en font très-grand cas; et quand nous en prenons en vie, nous les conservons ordinairement pour en faire présent au Capitan Pacha, lorsqu'il paroît avec sa 
flotte dans l'Archipel. Quand les paysans apercoivent un perdreau blanc, ils s'assemblent, le poursuivent sans relâche, et l'ont bientût lassé.

Il s'enfonce alors dans les brousșailles, où à l'aide d'un chien, on s'en empare. L'expérience apprend que si on tarde à le chasser, on ne le retrouve plus, les éperviers l'attrappant très-aisément. En voici la raison : Cet oiseau, vise bien sa proie, avant de fondre sur elle; mais dès qu'il s’est élancé, il ne peut plus changer de but : c'est pour cela qu'il ne se jette jamais sur une compagrie de pigeons on de perdrix de plumage uniforme, parce que, malgré la finesse de sa vue, le moindre mouvement entre eux opère une confusion qui l'embrouille, et lui fait manquer le but, sur-tout, sils sont bien serrés les uns contre les autres : aussi l'instinct porte-t-il ces oiscaux, quand ils voicnt un oiseau de rapine à se rapprocher, soit en volant, soit même parterre, le plusétroitement possible; et si celui-ci les attaque dans cet ćtat, ce n'est que pour les éponvanter, et parvenir à en détacher quelqu'un de la bạnde, dont il fait sa proie. J'ai été plusienrs fois témoin de cette ruse de la part des milans. Maiss'il y a dans la bande un pigeon ou une perdrix de couleur différente 
SuR L'île de Syra. Cha P. X. 225 des autres, l'oiseau chasseur n'est plus embarrassé et ne craint plus de perdre de vue sa proie; il fond sur elle sans chercher à la séparer du reste. C'est pour cela que les perdrix blanches, ou les pigeons blancs, parmi les ramicrs, disparoissent en peu de temps.

$16^{\circ}$. Nous avons à Syra un moyen infaillible pour connoître et remettre les luxations des membres des enfans. Quand aux cris et aux pleurs extraordinaires d'mn enfant, on soupçonne qu'il a quelque os de déboîté, ou, comme

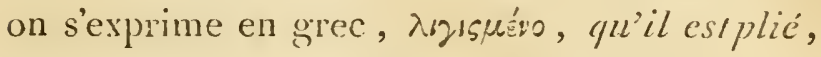
on l'étend sur un lit, le visage en dessous; on lui prend la main gauche et le pied droit, la main droite et le pied gauche, que l'on rapproche l'un de l'autre; s'ils se touchent aisément, et sans que l'enfant donne de plus grandes marques de souffrance, c'est uie preuve qn'il n'a rien de dérangé; si, au contraire, il ressent de fortes donleurs, il n'est plus question que de chercher l'endroit où la luxation a lieu. Pour cela on prend un jaune d'ouf que l'on pose très-doucement sur le dos de l'eufant, en le soulevant tantôt d'un rôté, tantôt de l'autre. On fait coulcr l'cuf sur tout son corps, depuis les reins jusqu’aus . épaules. Tome $I$. 
La place, où la pellicule qui enveloppe le jaune d'œuf se crève, est celle de la luxation. On procède à y remédier, et toutes les sagesfemmes, beaucoup de mères de familles même, ont ce talent. Je ne sais comment, dans un pays aussi éclairé que la France, et chez ses voisins, on n'a rien fait pour étendre une connoissance si simple et si utile; ce seroit le moyen de diminuer infiniment le nombre des personnes bossues, boiteuses, estropiées de dilï̌rentes façons par suite de luxation. Aussi n'ai-je connu, dans toutel'île de Syra, qu'une demoiselle contrefaite, et ce fut par la faute de sa nourrice, qui l'ajant laissée tomber clans son enfance, n’en avertit pas sa mère, et l'enfant infortuné resta boiteux.

Nous avons dit ailleurs qu'on fait à Syra de très belles toiles de coton croisées, dont tout le peuple ést habillé. Dans cette île, ainsi que dans toutes les autres de l'Archipel, on a une manière particulière de les préparer, avant de les mettre à la teinture. Pour les teindre en noir on les fait bouillir daus des écorces de grenade. Les femmes s'occupent de cette opération dans leur ménage, et en habillent assez. ordinairement leurs maris et leurs enfans. Je 
SUR L'île DE SYra. ChaP. X. 227 présume que les écorces de grenáde pourroient remplacer en teinture la noix de galle. On pourroit en faire des chargemens considérables dans tout le Levant, sur-tont du côté de Smirne, oü les grenades sont cn. grande quantité, et du côté d'Alexandrette, où il vient, de lintérieur du pays, une grande quantité de pepins de grenades aigres: les habitans de ces contrées s'en servent à la place du citron et du viuaigre.

On a encore dans le Levant une manière particuliere de guérir les érésipèles: on applique quelques pièces d'or sur la' partie malade; au bout de trois oin quatre heures on les retire, et on les essuie; on les remet encore sur la même partie, et en vingt-quatre hemes, l'or a attiré l'humeur, et l'érésipele a disparu.

Avant d'appliquer les prèces d'or sur l'érésipèle, on ne doit y employer aucune espéce d'onguent. On dit que la graisse, en bouchant les pores de la peau, empêche la vertu attractive de l'or.

15\%. Nous nous servons à Syra d'un levain particulier pour faire le pain. La veille du jour quon reutle pétrir, on prend environ me ou deux poignées de pois chiches, que l’on écrase: ensuite on les met dans l'cau chaude, mais 
non-bouillante, et dans un pot de terre, que l'on couvre avec du coton pour le tenir chaudement pendant toute la nuit. On le laisse ainsi jusqu'au lendemain; on passe cette infusion dans sés doigts; le suc et sur - tout l'écume s'écor lent et les pois chiches que l'on presse un peu restent. Avec cette infusion, et en y ajoutant une certaine quantité d'cau tiede, on mouille un peu de farine, et l'on forme une pâte un peu molle qu'on laisse pendant quelque temps (et ce temps est connu de tous les boulangers', bien courerte pour la tenir chaudement afin de la faire lever. Cette pate étant parvenue à la perfection du levain, on la mêle avec la farine qu'on veut pétrir.

Ce levain a beaucoup plus de ressort que le levain ordinaire. Il est plus sain et donne un goût excellent au pain, soit d'orge, soit de froment. Aussi le pain qu'on mange à Syra estil meilleur que dans les autres îles. Les biscuits, sur-tout, sont délicieux, et fondans, lorsquils sont faits avec attention : ce levain se multiplie, en conservant toujours me petite quantité de la même pâte de la veille pour le lendemain; cependant, pour lui conserver un meilleur goût, il faut renouveler 
SUR L'ille de Syra. Cha P. X. 229 de temps en temps le levain, comme nous l'avons dit.

Je crois ce levain très - propre pour le pain de mais, de pommes de terre, de châtaignes, etc.; l'essai en a été fait. Il est certain, que le pain d'orge fait avec le levain ordinaire, n'est pas mangeable; il est lourd et mat; celui, au contraire, qui est fait avec le levain des pois chiches est très-léger et bien levé.

Je terminerai ce chapitre par une anecdote singulière, qui peut faire quelque plaisir à nos lesteurs. Un ex-Jésuite Napolitain, appelé le Père Roxas, avoit élevé un 'aiğlon qu'il avoit pris dans son nid; il l'avoit tellement apprivoisé, quiil lui laissoit la plus grande liberté pour faire ses voyages aériens; aussi l'oiscau n'en abusoit-il jamais, et revenoit-il fort exactement, après avoir fait les plus longrues promenades. Il avoit pris un si grand attachement pour son maître, que toutes les fois que le père sortoit, il le suivoit en volant sur sa tête, pour savoir de quel côté il dirigeoit ses pas; et qu'aussitôt que le Père Roxas sarrêtoit pour s'asseoir, il descendoit des airs pour se mettre à côté de lui. Ce Père avoit une maison de campagne, où il alloit souvent. L'aighte 
230 Pr ÉCIS HÍTORIQUE ne manquoit jamais de le suivre; il n'entroit et ne sortoit qu'avec lui; si sun maitre étoit allé à la promenade pendant son absence, il prenoit l'essor et voloit à sa poursuite. Le P'ere Roxas n'avoit quà faire flotter un morceau de drap ronge, et l'oisean se précipitoit du lant des airs à côté de lui, et avec une si grande impétuosité, qu’il étoit obligé de se mettre à couvert sous un arbre, ou sous quelque autre abri, pour ne pas être terrassé; enfin ce singulier animal disparut un jour, et on n'en a plus entendu parler; il est vraisemblable qu'il fut tué. Il commencoit à faire de grands ravages dans l's!e ; il fondoit sur les troupeaux de moutons, et les dispersoit: la maison des Jésuites étoit continuellement assiégée de ceux qui venoient se plaindre des vols de cet aigle, et le Pére Ruaras les resituoit, ou en payoit la valeur. 
- sur l'île de Syra. Chap. Xi. 23 a

\section{C $\mathrm{H}$ A $\mathrm{P}$ I $\mathrm{T}$ R $\mathrm{E}$ X I.}

Méthode de caprifier le figuier, zısilée à Syra et dans loule la Grèce, depuis les lemps les plus reculés.

L a méthode de caprifier le figuier paroît remonter à des époques très-reculées. Théophraste (1), Pline (2), Valerius Cordus en ont parlé. Amasias, prêtre de Béthel (3), ayant repris Amos de ce qu'il prophétisoit des choses fâcheuses contre Israël, celui-ci lui répondit Je ne suis ni Prophete, ni fils de Prophète; mon occupation est de conduire mes troupeaux, et de picpuer des figues saurages.

(1) Lib. I, de causis plant. cap. 12.

(2) Hist. nat. l. 15, cap. 19, lib. 16, c. 27.

(3) Amos Proph.cap. vil , v. IX. Non sum propheta, et non sum filius propheta, sed armentarius ego sum vellicans sycomoros. 
Les écrits des anciens sur cet objet, ne présentent à l'esprit que des doutes et des conjectures purement idéales. Tournefort est le premier des modernes qui en a donné une idée claire et précise. Ce Savant est d'autant plus digne de foi, qu'il a parcouru toutes les îles de l'Archipel, et qu'il n'a rien avancé clont il n'ait été témoin oculaire.

Le procédé de la caprification mérite touce l'attention des Naturalistes. Snivi dans ses plus petits détails, il peut les conduire à des décou. vertes lumineuses sur la lécondation des plantes.

Syra est anjourd'hui le pays de la Grèce, et peut-être de l'univers, où, à proportion de son étendue et de sa population, on cultive une plus grande quantité de figuiers. C'est dans cette ile qu'on pratique la caprification avec le plus d'attention, d'inteligence et de méthode. J'y ai observé, pendant plusieurs années consécutives, la marche de cette opération; je crois donc me rendre agréable au public, et sur-tout aux amateurs, en rassemblant icice qu'en ont dit 'Tournefort, Linné et autres modernes. J'y ajouterai quelques particularités qui ont échappé à leurs observations, et je me flatte que ce 
SUR L'île de Syra. Chap. XI. 233 recueil renfermera tout ce qu'on peut desirer de certain et de probable sur cette matiére.

"Pour bien comprendre cette manufacture " de fignicrs ( la caprification), dit Tournefort "( Voyage au Levant, t. I , p. 338 ), il fant re"marquer que l'on cultive dans la plupart des "îles de l'Archipel, deux sortes de figuiers; la "première s'appelle ornos, lu grec littéral eri"nos (I), figuier sauvage, ou le caprificus des "Latins; la seconde espèce est le figuier domes" tique. Le saurage porte trois sortes de fruits, "Sornitès, cratitivès, orni, absolument néces"saires pour faire mûrir ceux des figuiers do"mestiques.

"Ceux qu'on appellefomitès, paroissent dans "le mois d'août, et durent jusqu'en norembre " sans mûrir; il s'y engendre de petits vers, " d'où sortent certains moucherons qu'on ne voit "voltiger quautour de ces arbres. Dans les mois " d'octobre et de novembre, ces moucherons pi"quent eux-mêmes les seconds fruits deś mê-

(1) Les anciens prononçoient erinos. L'usage ì consacré le mot onios par syncope; cependant plusieurs disent ernos et même erinos. On sait d'ailleurs que les Grecs substituoient s ourent une lettre à ?a place d'une autre. 
$23+$ PRECISHISTORIQUE

" ines pieds de figuiers. Ces fruits qu'on appelle "cralitirès, ne se montrent qu'à la fin de sep"tembre, et lesfomiles tombent pen après la " sortie de leurs moucherons. Les cratitirès, au " cuntraire, restent sus l'arbre jusqu'au mois de " mai, et renferment les wufs que les mouche" rons des fornites y ont déposés en les piquant: " dans le mois de mai, la troisième espèce de fi"gucs, commence à pousser sur les mêmes pieds "des figuicrs sauvages qui ont produit les deux "autres; ce fruit est beaucoup plus gros, et " se nomme omos. Lorsqu'il est parvenu à une "certaine grosseur, et que son wil commence "à s'entr'ouvrir, il est piqué dans cette partie "par les moucherons des cratitirès, qui se trou"vent en état de passer d'un fruit à l'autre, "pour y déposer leurs œufs.

" 11 arrive quelquefois que les moucherons " des cratitirés tardent à sortir dans certains "quartiers, tandis que les orni de ces mêmes " quartiers sont disposés à les recevoir (1). On

(1) Cela n'arrive jamais dans aucune partie du Levanl. On y remarque constamment que lorsque les orni sont disposés à receroir les cratitirès, ceux - ci sont déja mûrs, el lenr moucherons s'envolent. Míais ce yni pent 
SUR L'îl E DE Syra. ChAP. XI. 235 " eșt obligé, dans ce cas là, d'aller chercher " les cratitirés dass un autre quartier, et de les "ficher à l'extrémité des branches des figuiers "sauvages, dont les orni sont en bonne dispo"sition, afin que les moucherons les piquent: "si l'on manque ce temps, les orni tombent, " et les moucherons des cratitirès s'envolent.

"Il n’y a que les paysans, appliqués à la cul" ture des figuiers qui connoissent les momens, "pour ainsi dire, auxquels il fant y pourroir, " et pour cela, ils obserrent avec soin l'ocil de "la figue; non-senlement celte partie mar"que le temps où les piquems doirent sortir, " mais aussi celuj où la figne doit ćtre piquée " avec succès. Si l'veil est trop duret trop serré, "le moucheron ne sauroit déposer ses cul's, " et la figue tombe quand cet ceil est trop ou"vert.

"Ces troissortes de fiuits ne sont pas bons

arriver, c'est que les figuiers saurages marquent arant le : cratitirès et dans ce cas, pour empêcher la chûte des orni, on ramasse des cratitirès sur-un arbre qui en eș fourni, et on lez porte sur cę foguiers șaurages. 
« à manger : ils sont destinés à faire mûrir les

" fruits des figuiers domestiques. Voici l'usage "qu'on en fait : pendant les mois de juin et de "juillet, les paysans premnent les orni dans le "temps que leurs moucherons sont prêts à sor« tir, et les vont porter tout enfilés clans des fé«tus sur les figuiers domestiques; si l'on " manque ce temps favorable, les orni tombent, « et le fruit du figuicr domestique, ne mûris« sant pas, tombe aussi dans peu de temps. Les "paysans connoissent si bien ces précieux momens, que tous les matins en faisant leur revue, "ils ne transportent sur les figuiers domesti"ques que les orni bien conditionnés, autre" ment ils perdroient leur récolte. Je suis * forcé de dire curon n’a jamais vu employer à «Syra, l'axcolimbros, pour eaprifier le figuier. «D'ailleurs, cette méthode seroit absolument " inutile, puisque, comme je le prouverai, d'a"prèsinné, il ne suffit pas de piquer la figue, " mais de la féconder. Il est vrai qu’ils ont en" core une ressource quoique légère, c'est de "répandre sur les figuiers domestiques, l'ax"colimbros, plante très-commune dans les îles, " et dans les fruits desquels il se trouve des "moucheions propres à piquer; peut-être que 
SUR L'île de Syra. Chap. XI. 237 " ce sont les moucherons des orni, quii vont " picorer súr les fleurs de cette plante. Enfin, "les paysans ménagrent si bien les orni, que " leurs moucherons font mûrir les fruits du fi" guier domestique, dans l'espace de quarante "jours.

"Je ne pouvois assez admirer la patience des "Grecs, occupés pendant plus de deux mois "à porter ces piqueurs d'un figuier à l'autre. « J'en appris bientôt la raison : un de leurs ar« bres rapporte ordinairement jusqu’à 280 livres " de figues, au lieu que les nôtres n’en rappor« tent pas 25.

"Les piqûres, dit ailleurs Tournefort, con" tribueront peut-être à la maturité des fruits " du figuier domestique, en faisant extravaser "le suc nourricier, dont ils déchirent les ca"naux en déposant leurs œufs. Peut-être aussi, "qu'ontre leurs œufs, ils laissent échapper «quelque figuier, propre à fermenter douce« ment avec le lait de la figue et à en attendrir »la chair (1). Nos figues en province, et à Paris

(s) On pourroit peut-être dire, que les inoucherons éclos, ne peuvent subsister sans nourriture, et elle ne peut étre autre que les poussières des étamines des orni; 
" même, mûrissent bien pluś tôt. Si on pique "leurs yeux arec une paille graissée d'huile "d'olive, les prunes et les poirés piquées par "quelque insecte, mûrissènt plus tôt àussi, et " la chair d’ántour de la piqûre est de meilleur "goût que le reste (I). Il est hors de doute, "qu'il arrive un changement considérable daas "le tissu des fruits piqués, de même qu'il ar« rive aux parties des animaux, percées arec "quelque instrument.

Il ne seroit guere possible de bien entendre les anciens Auteurs qui ont parlé de la caprification, si l'on n'étvit pas convaincu des circonstances qui servent à la faire réussir. Non - seulement ce détail nous à été confirmé à Zéa, à Tine, à Scio, à Mícone, mais clans la plupart des autres îles de l'Aıchipel.

ces poussières sortant ensuite en excrément du corps de ceux qui sont entrés dans les figues femelles, ont la vertu de les féconder.

(i) La partie d'une prune piquée par un insecte, ne reçoit pas toute la sève quilui est destinée. Cette surabondance, refluant sur tout le fruit, doit nícessairement hâter sa maturité, et lui donner un gon̂̊ plus agréable. 
Sur l'île de Syra. Chap. XI. 239

On ne peut rien désirer de plus satisfaisant que cet extrait de l'ourrage de Tournefort; il est à remarquer quill soupçonnoit seulement avec Valerius Cordus, que les figues avoient des fleurs; mais ni lui, ni diantres n'avoient encore connu les vraies parties de ces fleurs, jusqu'à l'année 1712 , que $M$. de la Hire a dérouvert et démontré publiquement leurs étamines", et leurs sommets couverts d'une poussière très-fine. Je joindrai ici quelques particularités, fruits de mes observations; elles contribueront à rendre plus intelligible le procédé des Levantins pour la caprification.

Les paysans ne sont pas les seuls à Syra qui s'appliquent à la caprification; elle est pour bien des personnes, un objet d'amusement. J'ai enfilé moi-même dans du jonc des figues mâles des orni, que j’ai déposées sur des figuiers domestiques. Quelquefois ourrant ces orn; ou érini, j’ai reconnu que tous les grains étoient comme autant d'œufs, qui renfermoient un petit moucheron; je m'en suis assuré en écrasan $t$ plusieurs de ces grains, lorsque la figue mále commençoit à s'entr'ourrir, et constamment jai trouvé un mouclieron dans chaque grain; lors. que l'essaim de ces petits moncherons n'étvit 

point entièrement éclos. J'en ai observé plusieurs couvertes d'une poussicre fine. (L'usage dé secouer ces sortes de figues, avant de le $e^{\mathrm{s}}$ manger, pour en faire sortir la poussière, ne laisse aucun doute sur ce fait). J'ai répété la même expérience sur dés orni, après leur entière maturité; tous les grains étoient percés, les moucherons en étoient sortis, l'enveloppe seule des grains restoit.

Cette déconverte auroit présenté aux anciens Naturalistes, une question intéressante à résoudre; savoir, si les végétanx penvent produire des insectes quelconques. Quoi quil en soit, je pense que le fait précédent duit être expliqué de la maniere suivante : les petits moucherons sortent des cratitirès pour entrer dans les orni, lorsque les grains en sont encore tendres; its les percent et y déposent leurs cuf́s; de ces œuls, éciosent bientût de petits vers, qui devenus moucherons, sortent vraisemblablement, par les mêmes perits trous, qui aroient scrvi à leurs mères pour y déposer leurs œufs.

Il.enest de même des moucherons des fornitès, qui déposent leurs oufs clans les graines des cratitirès. Les premiers ont été nécessaireuicut produits par d’autres moucherons. Quelle 
SUR L'île DE Syra, ChaP. XI. 241 est leur origine? quelle en est la cause? je l'ignore; tenons-nous-en toujours au grand principe, omme vioum ex ovo.

J'invite les amateurs et les curieux à constater ce fait par le secours des instrumens, et d'aualyser, pour ainsi dire, les grains des orni, après leur caprification, et même à diverses époques jusqu’à celle de lcur maturité. Je ne donte pas qu'ils n'y trouvent d'abord des oufs, puis de petits vers, et bientôt des moucherons.

Les sociétés des savans ne pourroient-elles pas confier le soin de cette expérience à nos voyageurs au Levant, ou à des personnes éclairées, résidantes à Constantinople ; Smirne, et dans les îles de l'Archipel, qui leur adresseroient les résultats de leurs observations?

En voici quelques-unes que j'ai faites moimême, et qui pourront donner des lumières sur la caprification. J'ai cueilli quelquelois des omi dans un mouchoir blanc, dans le dessein de les placer sur des figuiers domestiques. Quand je les en retirois, le mouchoir restoit couvert de petits moucherons: je l'étendois surles branches d'un figuier; un instant après ils avoient disparu.
Ordinairement il n'entre qu'un seul de ces Tome $I$. 
moucherons dans cluaque fruit, ce dont je me suis assuré plus d'une fois, en approchant un de ces insectes d'une figue déja occupée par un autre; il ne s'y arrêtoit point, et voltigeoit de branche en branche. Je le posois ensuite à l'oil d'une figue qui n'étoit point encore habitée; alors il en soulevoit les écailles avec son museau, et se frayoit une route, par laquelle il s'y introduisoit en laissant ses ailes en dehor's, suivant l'usage de ces insectes; car j'ai observé, sur plusieurs figues, les ailes de ces petits animaux, serrées entre les écailles. Quelquefois, mais raremẹnt, j'ai vii, àl'œil d'une même figue, pluscle deux ailes; ce qui me porteroit à croire que plusieurs moucherons y avoient pénétré; ce que la petitesse et l'extrême mobilité de l'insecte mont empêché de vérifier. Les écailles de la figue sont très-serrées jusqu'au moment de la fleuraison ; alors elles s'entr'o u vrént et se soulèvent l: dès que le temps de la fleur est passé, clles se resscrrent, de sorte qu'il est impossible aux petits moucherons d'y pénétrer ; c'est pour cela que l'on guette cet instant si précieux pour la caprification ; car autrement la figurue sèche, et se détache de l'arbre avant sa maturité. 
SUR L'île de Syra. Chap. XI. $24^{3}$

D'après tout ce que nous venons de dire, on voit que Tournefort'est dans l'erreur, lorsqu'il avance que la caprification s'opère par les piqûres des moucherons. Une fois que le petit moucheron est parvenu à soulever les écailles, il tronve un passage librè pour pénétrer dans la cavité de la figue, sans piqûre et sans blessure. Cette observation nous servira par la suite pour répondrè à quelques difficultés.

Les cultivateurs ont remarqué que lorsqu'ils opéroient à propos la caprification des premières figues, celles qui fleurissoient après, étoient facilement caprifiées; ils éprouvoient le contraire lor'sque, par négligence ou autre cause, ils manquoient le temps convenable pour les premières.

D’après les observations qui précèdent, il est aisé de connoître le moment propre à la caprification, celui où elle devient impossible, enfin celui où elle est opérée. Dans le premier cas, lcs écailles sont un peu soulevées et entrouvertes; dansle second elles sont bien resserrées, de manière que l'oeil de la petite figue se trouve un peu enfoncé, et dans le troisième, clles paroissent comme avant le temps de la fleuraison; mais l'œil est saillant, quoique plus rétréci. J'ai déja dit que ce temps est le scul propre 
à la caprification. Les Grecs l'appellent cuthimisis tis sikias, le souvenir du figuier : ce dernier motest le tcrme générique dont ils se servent pour exprimer le moment de la fécondation de tous les êtres animés, et ils y ajoutent la signification particulière de chacun; ainsi ils disent: Iagheladha enthimare, i foradha enthimate, to prosato enthimate, pour dire qu'une vache, une jument, une brebis est disposée à être fécondée. Ces expressions, tirées de leur langue, prouvent quils avoient une idée de la fécondation des plantes: peut-être sommes-nous plus ignorans que ce peuple sur cette matiere; et même il paroît que quelques anciens Philosophes Grecs avoient connu que cette fécondation s'opéroit par le moyen des poussières des étamines des fleurs mâles qui pénètrent dans les pistils des femelles, ainsi que nos Naturalistes croient l avoir découvert. En effet Pline, cap vir, hist. nat. lib. 2 , rapporte le sentiment de Ménécrate, qui croyoit qu'cllcs étoient un indice de Ja prochaine récolte des fruits. Mencerates, dit-il, florem (c'est-à-dire, la poussière des étamines ) esse dicit, funure messis indicium ; sed nemo prater enm : mais il ajonte que c'étoit une opinion particulicre de Ménécrate; que gersome après lui ne la suivoit. 
SUR L'île de Syra. Chap. XI. 245

Lorsque la figue sauvage est à l'instant de sa maturité, son ceil s'ouvre entièrement, ses moucherons sortent librement, et ils s'envolent sur les figues domestiques.

Il y a à Syra quelques espèces de figuiers qui se caprifient plus aisément: quelle en est la cause? c'est que leurs figues restent plus longtemps en fleurs, tandis que la fleuraison des autresne dure, pour ainsi dire, qu'un seul instant, Si les Cultivateurs le manquent, la caprification ne peut avoir lieu, et les fruits tombent avant leur maturité.

Plusieurs espèces n'ont pas besoin de la ca. prification; tclle est celle qu'on nomme stournailia, dont les fruits mûrissent dans le mois d'octobre et de novembre; et même pendant l'été ; il fant nécessairement regarder ces figuiers comme des hermaphrodites. On les appelle sauvages, parce qu'ils viennent sans culture sur les montagnes et au milieu des rochers, de grains transportés par le vent ou par des ani maux; et leurs finits sont bien différens de ceux que l'on cultive communément dans l'île.

Quelques autres espèces d'orni offrent des particularités intéressantes, mais que je ne rapporte point ici, faute d'instruclion et d'unc étude

Q iij 
246 PRÉCIS HISTORIQUE assez suivie. Un Grec, natif de l'île de Patmos, et qui se trouve actuellement à Paris, m’a dit avoir dans son jardin denx figuiers sauvagges, qui ne produisoient que des cratitires.

Enfin, on trouve à Syra un seul arbre d'une especce de figues, qu'on appelle arbicannes; cette espèce a été apportée de l'Italie à. Tine, et de Tine à Syra : elle est cultivée daus tous les jardins potagers que jài vus à Rome; elle porte du fruit deux fois l'année, dans le mois de mai et le mois d'août. Les premières figues sont plus grrosses, et d'un meilleur ģoût. Les unes et les autres n'ont pas besoin de caprification pour parvenir à leur maturité ; peut-être même pourroit-on croire que les sccondes sont caprifiées par les premières.

Lcs curieux demanderont sans donte ce que devient le moucheron qui pénetre dans la figue domestique, et qui semble la féconder et hâter sa maturité? J'avoue que je n'ai fait, sur ce point ancune recherche particulière; mais, ne pourroit-on pas, avec un microscope, parvenir à cette découverte intéressante, en observant plusieurs figues à diverses époques, jusquà celle de leur maturité? Ce que jc puis avancer avec certitude, cest que, lorsqu'on les ouvre pour les manger, 
sur l'ile de Syra. Chap. XI.

on ny découvre ni moucheron, ni œuf, ni ver. Mais, lorsqu'elles sont sèches, et qu'on les entasse dans des vases, sans les faire passer par le four, on observe dans chaque figue un seul ver, lequel est nécessairement engendré par l'œuf que le moucheron de l'ornos y a déposé en y pénétrant. J'ai aperçu quelquefois une espèce dẻ tissu soyeux dans ces figues sèches; ce qui me fait présumer que mon moucheron est un ichneumon, comme l'a soupçonné Linné; car les ichneumons ont cela de commun avec les tetraptères à ailes farineuses, qu'ils se filent une espèce de coque, et sur-tout les ichneumons à coton. J'ai eu occasion, de remarquer d'ailleurs que la larve étoit mollasse, blanchâtre, sans pattes, seulement avec une tête brune et écailleuse: l'irsecte parfait, beaucoup plus petit, s'est refusé à mes observations.

Cỏmment ce moucheron a-t-il pu être féconclé et produire cet œuf? Je pense que cet insecte est déja fécondé lorsụiil sort de la figue sauvage; car j’ai remarqué qu'il reste dans cha cune plusieurs moucherons morts, qui sans doute sont les mâles qui ont opéré la fécondation; parce que jesuppose que ces moucherons meurent après avoir fécondé les femelles, ainsi qu'il arrive à 
plusieurs antres insectes, sur-tout aux faux bourdons (qui sont les mâles dans la république des abeilles), comme nous le verrons dans motre traité sur ces insectes.

Les figues que l'on n'expose point à la chaleur du feu, renferment toutes un ver: aussi dans l'Archipel a-t-on soin d'user de cette précaution, non pour les faire sécher, comme dit Tournefort, mais pour étouffer le gêrme du ver qui ne tarderoit pas à éclore.

Quclques personnes se contentent de la presser dans des pots de terre, que l'on couvre avec soin hermétiquement, pour interrompre l'action de l'air extérieur, et ce procédé produit le même effet.

Avant de passer à l'opinion de Linné, il est à propos de remarquer qu'il a admis des fleurs hermaphrodites dans le figuier, aussi bien que des fleurs senlement mâles ou seulement femelles, puisqu'il classe les figuiers dans la polygamie; ainsi nous avons des figuiers hermaphrodites, tels que les sioumailia, et autres, étrangers par conséquent à la caprification, et des figuiers mâles et des figuiers femelles. Je repporte d'autant plus volontiers l'opinion de ce grand Naturaliste, qu'elle est entièrement 
SUR L'îlE DE SYR A. Chap. XI. 249 conforme à la mienne, et que l'une peut servir de preuve à l'autre. Voici comment il s'exprime: Vol. r. Amænit. Acad. p. 227.

"Il est certaines espèces de figuier's, dont les "fruits étant seulement remplis de pistils, ne "sont 'que des réceptacles. Ces figuiers sont " conséquemment feinelles; et Pontedera leur " donne proprement le nom de ficus : on les " trouve dans nos jardins, et ils donnent une "grande quantité de fruits; d'autres au con" traire renferment le germe du fruit; ils en "sont le réceptacle, et produisent de petites "figues. Ce sont les figuiers mâles auxquels "Pontedera, et les anciens ont donné le nom de « caprificus. Ceux-ci ne sont pas cultivés dans " nos jardins, parce que, dès que leurs petites "figues ont atteint la moitié de la grosseur or"dinaire des figues, et que les rents ont em"porté leur poussière, elles tombent avec "leurs réceptacles, avant de parvenir à leur " maturité.

“ D'autres figuiers produisent au printemps, " comme le caprificus, des petites figues qui don* nent leur poussière, et tombent avant leur "maturité :à l'automne suivante ils en produi"sent de nouvelles avec des pistils; celles-ci 
" mûrissent au printemps suivant. Pontedera " appelle cette dernière espèce de figues, sri* nasyces, et elles ne sont réellement autre "chose que des androgynes ou hermaphro"diles.»

Ce que dit ici Linné, paroîtroit fort obscur, si ce que j’ai rapporté de 'Tournefort, et mes observations particulières qui l'accompagnent, ne nous en donnoient une explication satisfaisante: au reste, toute cette exposition de Linné, on doit l'entendre conformément à ce que jai exposé ci-dessus. Je remarque seulement que les figues mâles, les orni, par exemple, ne tombent que parce qu'elles n'ont pas été caprifiées par les cratitirès : celles qui l'ont été, mûrissent, et ne se détachent de l'arbre qu’après avoir donné leurs moucherons et leurs poussières. Il y a, entre ces figues mâles ou orni, quelques espèces qui sont bonnes à manger; leur goût est agrréable : presque toutes les autres espèces ont un degoré de douceur, et les cochons en sont fort friands.

Linnéc prétend que l'on cultive en Hollande et en Suede des figuiers femelles qui produisent une grrande quantité de fruits ; cela est étonnant, puisque ceux que l'on voit à Paris, quoi- 
SUR L'île DE Syra. Chap. XI. 25 r que situés dans une latitude plus favorable, sont des figuicrs sauvages qui produisent trèsrarement, et fort peu de fruits.

"Peut-être, poursuit Linné, tronve-t.on " d'autres espèces de figuiers, dont les fruits, "dans un seul et même réceptacle, renferment " des fleurs mâles et femelles, dont les unes "sont placées dans leurs cavités, et les autres " entre leurs écailles, si touiefois l'on doit s’en "rapporter à la description et aux figures de «M. de la Hire ( $r$ ).

«Ce qui me feroit croire cependant que ces " espèces sont très-rares, c'est qu'aucun Natu" raliste, jusqu'à nos jours, n’en a donné une "description exacte, qu'on ne les cultive pas " dans nos jardins, et qu'aucun de nous, même

(s) Linné pose en assertion dans son genera plan1arum, $p .776$, ce qu'il vient de ne poser ici que d'une manière douteuse. "Le calice des fleurs, (dit-il), est "commun ou plutôt c'est la figue elle-même. Sa sur" face interne est converte de petites fleurs, dont les ex" térieures, ou les plus proches des éeailles qui la fer " ment à sa surfacc supérieure, sont les fleurs mâles qui " sont en petit nombre, et au-dessous de celles-là, les " fleurs femelles en très-grand nombre, ” 
" l'illustre Président de cette Académie, n’en " ont jamais vu. Si leur existence est réelle, on " cloit les appeler hermaphrodites."

D’après ce sentiment, on doit penser que les figuiers qui, dans l'Archipel, n'ont pas besoin de Ja caprification, sont hermaphrodites, tels que ceux dont parle Linné, d'après $M$. de la Hire; par la même raison, ceux qui donnent du fruit deux fois dans la même année, au mois de mai et au mois d'août, sont de vrais hermaphrodites, ou parce qu'ils portent deux espèces de figues, les mâles qui sont celles du mois de mai, etles femelles, cellesdu mois d'août. En ce cas il faut, ou que les premières fécondent les secondes, ou quie les mêmes figues renferment dansleur réceptacle, desfleurs femelles et mâles. Tels sont peut-être les figuiers que l'on voit en Italie et dans les provinces méridionales de la France. Cependant cela s'opposeroit à ce que nous rapporterons ci-dessous, "d'après le même Linné, qui atteste que les grains de figue des pays où la caprification n'a pas lieu, semés, ne poussent aucune plante, parce qu'ils ne sont pas fécondés: or, si ces figues étoient hermaphrodites, leurs grains devoient être aussi bien fécondés que ceux, des figues caprifiées. 
S UR L'île DE SyRA. Chap. XI. 253

"La caprification est un procéclé que les " anciens clepuis les temps lesplus réculés, ont " constamment pratiqué dans la culture du "figuier, et qui se pratique encore régulière. "ment chaque amnée dans les îles de l'Archipel " et clans les autres îles de la Grèce. Pline, "Théophraste, Suidas, Plutarque, et autres au"teurs célèbres en font mention; le premier, "sur-tout, nous en a laissé une idlée précise, « lib. 15.

"Le caprificus, dit - il, est un figuier sau"vage, dont les fruits ne mûrissent jamais; "mais il communique à d'autres, la propriété "qui lui manque, puisqu'il s'opère un passage «naturel des causes, et que par la putréfac" tion, il s'engendre un nouvel être, il produit " donc des moucherons. Ceux-ci, sans alimens " dans le sein de leur mère, s'envolent 'sur les " figuiers domestiques, et appaisant leur avi" dité par leurs piqûres répétées, ils percent "l'œil de la figue, y pénètrent, et ouvrent "pour la première fois, un passage aux rayons "clu soleil et aux vents qui dessèchent les hu" meurs abondantes. Bientôt ils absorbent le suc "laiteux qui est le premier rudiment du fruit. "Ceci arrive aussi naturellement; c'est pour 
" cette raison quédansles lieux plantés de figuiers, " on a soin de placerle caprificus sur le vent; afin " que son action dirige le vol de ces mouche"rons sur les fiquiers domestiques: de là est * yenu l'usage d'unir des figues sanvages les " unes aux autres, et de les porter sur un de ces " arbres. Cette précaution n'est pas nécessaire " dans les terrains secs et exposés au nord, parce " que l'air pompe naturellement le suc laiteux, " et occasionne le même effet que les mouche" rons ont produit à l'oeil de la figue. Il en est de " même des lieux où les vents élèvent beaucoup * de poussière, comme parexemple sur les bords * des chemins; cette poussière dessèche la fi- gue, et en absorbe l'humeur laiteuse. Elle a " le même avantage que la caprification; clle " empêche la chûte de la figue, en consumant " une humeur qui, quoique légère lui devien"droit inutile.

"C'estainsi que Jean-Bauhiuus renferme en* peu de mots, l’opinion des anciens sur la ca«prification r, p. r 35 . Des moucherons, dit-il, * engendrés par la pourriture du fruit du capri* ficus, s'envolent sur les figues domestiques. * Par leurs morsures, ils en percent l'oeil, s'y «nourrissent de l'humeur superflue, et y intro- 
Sur L'île de Syra. Chap. XI. 255 "duisent en même-temps les rayons du soleil, "de manière, que non - seulement, ils empê" chent leur chûte, mais encore ils excitent et "hâtent leur maturité. C'est pour cela, dit "Pline, que dans les lieux oú l'on plante les fi"guiers, on à soin de placer le caprificus sous "le vent, afin que son action dirige le vol des "moucherons sur les figuiers domestiques.

L'opinion de Pline, et celle de Bauhinus, sur la caprification, ne s'accorde pas entièrement arec l'expérience.

$\mathbf{I}^{\circ}$. Les moucherons ne sont point engendrés par la pourriture.

$2^{\circ}$. Puisqu'il ne s'introduit qu'un seul mou. cheron dans chaque figue, ce petit insecte ne, pent en consumer le suc laitenx qui est trèsabondant, et qui d'ailleurs a sa source dans le péricarpe, et non dans le centre où il fixe sa demeure.

$3^{\circ}$. Le moucheron pénètre dans la figue, en soulevant ses écailles, sans la piquer et la percer.

$4^{\circ}$. La poussière que le vent élève, n'a point la vertu de raffermir la figue à sa tige. Les figuiers, qui sont plantés à Syra sur les bords 
des chemins, ne conservent leurs fruits que par. le concours intéressant de da caprification.

$5^{\circ}$. Les rayons du soleil sont encore un moyen stérile pour exciter la maturité, puisque, dans le Levant, malgré leur brûlante arcleur, les figues non caprifićes tombent avant de mûrir. Il en est de même de l'exposition: de plus, on a remarqué que lorsque le vent du nord souffle pendant la fleuraison, la caprification ne s'opere pas avec un entier succès; les moucherons ne pouvant résister à sa violence, sont emportés au loin et très-maltraités.

L'opinion de Linné est donc la plus satisfaisante et la plus probable: suivons cet habile naturaliste dans ses détails.

"Nous avons dit ci-dessus, (ce sont ses paroles * traduites clu latin), que le caprifiguier étoit le « mâle, et le figuier cultivé, la femelle. Nous * avons aussi exposé comment les fleurs sont "renfermées clans la cavité du réceptacle, dont "les bords connivens sont tellement resserrés, "que l'on peut à peine passer la pointe d'une "aiguille par l'ombilic. Pour que les fleurs fe"melles soient fécondées, il faut nécessaire"ment que la poussière des anthères sorte de "la 
SUR L'île de Syra. Chap. XI. 259 « la fructification du caprifiguier soit emportée « par le vent sur les parties génitales du figuier « domestique, s'y introduisent par l'ouverture « étroite de l'ombilic, et se répandent dans toute « sa cavité.

«Cette exécution paroîtroit an-dessus des * forces de la nature, si le Créateur suprême "n'avoit assigné ì ce figuier son propre cupi"don. Ce cupidon n'est autre chose que ce que " les anciens ont appelé psenem, ou l'insecte du " figuier. Pontedera, dans son anthologie, par.: « 172 , nous en a donné la description et la " figure, fig. II. Cet insecte est le culex des an«ciens, et une espèce d'ichneumon que l'Être "tont-puissant a consacré, autant que nous « en avons pu juger , ¿̀ la fructification du figuier, " pour qu'il y dépose ses œuf’s, et y fasse éclore «ses petits.

"C'est ainsi que, parmi les insectes, le ver à * soie ne s'attache qu'au mûrier, l'ichneumon à a l'églantier, un autre au chêne, etc. Celui du "figuier a un ennemi particulier, qui se plaît * d'autant plus à en fairesa proie, qu'elle est " pour lui une nourriture délicieuse. En effet, * suivant le raport de Bauhirus, l'on voit croître «sur l'orme des montagnes, ainsi que sur le Tome $r$ : 
" pistachier et le peuplier noir, des follicules " produites par certains insectes qui détruisent " celuidu figuier, comme la mouche qui dévore "les apphides . "

L'on ne connoît point à Syra d'autres ennemis aux moncherons du figuier, que les fourmis. Celles-ci, dans le temps de la caprification, se placent, pour ainsi dire, en cmbuscade à l'œil de la figue, et y attendent leur entrée ou leur sortie; quelquefois elles sintroduisent dans le caprificus, et dévorent ces moucherons dans le scin même de leur mère.

"Ces ichneunons, après avoir subi divers " changemens, deviennent ailés; c'est alors que " le caprifgujer on le figuier mâle fleurit, et " laisse échapper la poussière des anthères. Ces " insectes sortent de la cavité du fruit, couverts " de cette poussière, comme un meûnier sor" tant de son moulin. Déja fécondés, leur seule " inquiétude est de déposer leurs œufs; ils s’at* tachent chacun à une petite figue ; et en en" trant dans la fructification, dont les bords "sont hérissés de pointes ou de pistils, ils "laissent nécessairement la poussière dont ils " sont enveloppés; c’est ainsi que s'opère la fécon"dation du figuier femelle." 
SUR I'ILE DE SYRA. CHAP. XI. 26E

"Cet arbre peut, àla vérité, conduire jusqu’à " maturité quelques-uns de ses fruits, parce qu'ils " ne sont que de simples calices, ou réceptacles, " qui contiennent les vrais grains. Cependant ces "grains ne seront jamais fécondés, et ils n'auront " jamais la vertu de produire des plantes de leur " espèce, sans le concours de la caprification. Il " en est dle même du houblon, du fraisier, du $\approx$ mûrier, dont les fruits ne sont aussi que des " calices. "

"Quelques Botanistes auxquels cette parti" cularité a échappé, voyant ces arbres produire " leurs fruits sans le concours préalable de la "fécondation, ont cru avoir trouvé un argu" ment irrésistible contre la génération des plan" tes; mais ils n'avoient point observé que le "fruit du figuier étoit un réceptacle commun "ou un calice, et non la graine qui doit fructi" fier. Il est certain néanmoins que la figue " dont les pistils ontété fécondés se gonfle da"vantage. C'est ce dont s'est assuré Tournefort; " puisqu'il rapporte qu'en France, où la capri" fication ne s'opère pas, un arbre ne donne tous " les ans que 26 livres de figues, tandis quin « arbre de la même grosseur en produit R ij 
262 PRÉCIS HISTORIQUE

“ à son cultivateur 280 livres, c'est-à-dire, un « nombre dix fois plus grand.

“C'est avec raison que notre siècle, oú la " botanique a fait des progrès étonnans, n'a« dopte point le sentiment de Camérarius qui " prétend que la semence du figuier est stérile : «il est réfuté par l'observation du très - célèbre "Président, rapportée par J. Browaillius, iro " examine epicreseos siegesbackiana elit. ult. « p. 16.

“ J'ai appris de Linné que tous les ans, er « Hollande, on se procure des plantes de " figuier en jetant à terre de la semence, et « même le fruit divisé en plusieurs parties, mais * qu'à la vérité, ce fruit étoit apporté d'Italie; * car la semence des figues produites en France, « en Allemagne, en Angleterre, et même en "Suède, où le caprifiguier n'est pas connu, est «stérile; au contraire, celle des figues qui ont * subi la caprification en Italie et dans l'Archi" pel, germe avec succès, et les feuilles des jeu"nes plantes sont dès les premiers jours sem« blables à celles de la mouche.

"Cette expérience a été répétée cette an" née au jardin de l'Académie. On a semé " dans des pots de terre des figues du Levant 
SUR L'île de Syra. Chap. XI. 263 « on les a abritées dans la serre, et on a vu pa« roître diverses plantes. Ceque dit Camérarius, « ne peut donc s'entendre que des figuiers non » fécondés; et désormais personne ne révoquera " en doute la reproduction du figuier par sa "propre semence."

Il est évident que les observations de $\mathrm{M}$. Linné sont entièrement conformes à celles que j’ai faites moi-même à Syra, et que j’ai rapportées ci-clessus. Nous nous accordons à penser, $\mathbf{1}^{\circ}$. que les figues mâles renferment une póussière, dont les moucherons sont couverts en sortant de la fructification.

$2^{\circ}$. Que cette poussière est portée par ces insectes sur les figues femelles, et féconde leurs pistils.

3. Enfin, qu'une figue peut atteindre à sa maturité, sans le concours de la caprification; mais outre qu'elle ne parvient pas à son entier développement, comme les figues fécondées, sa semence est stérile; selon Linné. Chez nous cependant les figues , qui mûrissent sans être caprifiées, (cequi arrive très-rarement) outre qu'elles sont très-petites, n'ont point de graines dans leur cavité; ces graines étant toutes avortées, faute 
264 PRÉCISHISTORIQUE d'être fécondées par l'opération de la caprification.

- D’après ce que nous avons exposé jusqu'ici, il est aisé de réfuter les objections de $M$. de l'Haire, auteur allemand, contre le systême de Linné. Voici ses propres paroles : Tab. 53,54 .

"Quoique les preuves que nous avons rappor" téessoient favorablesà la fécondationquis'opère " par les moucherons du figuier; cependant on " seroit en droit de douter si cet insecte fécon"de réellement avec la poussière dont sont " enveloppés les pistils des figues domestiques, " situés dans la cavité du réceptacle; puisqu'ils «ne peuvent y pénétrer qu'en se frayant, par "leurs piqûres répétées, un passage trop petit " pour leur corps, et par coinséquent, ils doi"vent laisser sur les bords de l'ail cette pous"sière; d'autant plus quele suc laiteux, qui " découle des tubes déchirés, la détrempe, et "que d'ailleurs les pistils qui se trouvent dans " la partie intérieure de la fiructification sont "situés moins favorablement pour être fécon" dés. Je croirois donc avec assez de fondement " que la fécondation ne s'opère pas par les mou" cherons qui s'introduisent dans le réceptacle. 
SUR L'íle de Syra. Chap. XI. 265

* Il est plus probable que ces insectes retour" nent au figuier sauvage, pour revenir encore " au figuier clomestique, ou que de nouveaux "moucherons, trouvant les bords de la figue "domestique desséchés par l'action de l'air " et 'du soleil, et un peu entronverts, y pé« nètrent avec la poussière fécondlante... " D’ailleurs je pense que l'air chargé des éta"mines contribue à la reproduction des plan» tes qui portent des pistils. C'est pour cela que " les figuiers domestiques, plantés dans un ter" rain sec, n'ont pas besoin de la caprification; " puisque leur receptacle, s'entrouvrant natu" rellement, donne entrée à l'air chargé de la "poussière des étamines.

"Les objections de cet auteur allemand, sont * suffisamment réfutées par l'opinion des Bo" tanistes les plus célèbres, et par mes observa" tions particulières. J'opposerai ici seulement " ses assertions erronées, à l'expérience.

$1^{\circ}$. Il est démontré que le moucheron ne pénètre pas dlans la figue par ses piqûres, mais qu'il en soulève les écailles déja entr'ouvertes par la végétation; d’où il suit, que le suc laiteux ne peut s'écouler par aucune partie blese 
266 PRECIS HISTORIQU E sée, ni produire aucun effet contraire à la cá prification.

$2^{\circ}$. Il suit de là que toutes les conjectures de motre auteur, sont fausses, sans aucun fondement et contraires à toute expérience; c'est-àdirc, que les moucherons retournent à la fructification du figuier sauvage, pour revenir sur le figuier domestique, couverts d'une nouvelle poussière ou que d'autres moucherons perfectionnent l'acte de la fécondation que les premiers n'avoient que préparé.

En effet, il est très-rare de voir plus d'un de ces moucherons entrer dans une figue femelle. Nous avons dit encore que souvent ces moucherons, en entrant dans ces mêmes figues, laissent leurs ailes à l'entrée. Il leur est donc impossible de retourner à la figue du caprificus. 3. Le concours de la caprification est ab. solument nécessaire pour féconder le figuier domestique; l'air, le soleil, le vent sont des moyens accessoires et insuffisans qui, comme nous l'avons dit, aident la maturité clu fruit sans lui donner tout le développement dont la fécondațion le rend susceptible.

$4^{\circ}$. Enfin, clans l'Archipel, on dessèche les figues au soleil. Si on les expose à unc cha. 
SUR L'île de SyRA. Chap. XI. 267 leur modérée, cette chaleur assurément n'est pas assez forte pour détruire la force prolifique des graines. An reste celles qui ont servi à M. Linné pour faire ses expériences étoient sans doute venues de Smyrne, d'où on en transporte une grande quantité en Hollande et dans le Nord. Dans cette partie du Levant, l'usage n'est pas de faire passer les figues au four, on se contente de les dessécher au soleil, et de les entasser avec ordre dans des caisses, que l'on ferme ensuite avec soin quand ellessont remplies. 


\section{H A PI TRE XII.}

Aloyen simple et facile dont se serwent les habituns de l'ile de syra pour guérir la jaunisse.

L E procédé qu'on emploie dans l'île de Syra pour guérir la jaunisse, a une vertu si prompte ct si universelle, qu'il doit engager tout Philantrope à en chercher la cause et en raisonner les effets. Ce moyen est trop répandu dans toutes les îles de l'Archipel, et sur - tout à Syra, pour qu'on puisse douter de son efficacité.

On met une pièce d'or dans un verre d'eau ou de vin blanc, on l'expose au serein, et l'on a soin de l'en retirer avant le lever du soleil. On prend cette cau à jeun, et trois ou quatre jours suffisent pour emporter la maladic. Il n'y a point d'exemple qu'elle ait résisté plus long-temps. Cette pratique est suivie du même succès à Constanti- 
SUR L'île de SYRA. Chap. XII. 269 nople (1). Nous y arons connu une personne, dont les parens habitent actuellement Versailles, qui ayant épuisé en vain toutes les ressources de l'art pour se délivrer d'une jaunisse invétérćc, ne put y parvenir qu'en usant de l'eau dont nous venons de parler. En trois jours elle fut parfaitement ğuérie, après avoir évacué par les sueurs une grande quantité d'humeurs jaune-foncé.

Personne jusqu'ici, dans l'île, n'a fait assez d'attention à cette précieuse recette; elle méritoit cependant quelques recherches sur la cause qui communique à l'eau, ainsi exposée , lefficacité de guérir la jaunisse. L'indifférence

(1) Une personne, digne de foi, m'a assuré que dans la Transilvanie, on emploie le même moyen pour guérir la jaunisse; excepté quele vase qui eontient l'eau avec la piece d'or, doit être enduit de cire jaune. A Alep on guérit aussi cette maladie, en exposant au se. rein une mûre saupoudrée de sucre que i'on mangeà jeun pendant quelques jours. En Espagne on pxpose au serein du pain trempé dans une eertaine quantité d'eau ; on l'exprime pour en retirer la liqueur; on la met sur un feu Jent; on y ajonte un jaune d'œuf, et ce mélange forme une espèce de lait de poule, qui est admirable pour la plithysie et la consomption. 
a été portée si loin, que personne n'a même pensé à employer ce remède à d'autres 'maladies auxquelles peut-être il auroit été salutaire. Cela proure à quel point le découragement de tous les peuples soumis à la domination des Ottomans les empêche de rien entreprendre.

D'après les effets admirables de l'eau d'or, (appelée ainsi du nom de son auteur) dont même nous avons été témoins en arrivant à Paris, nous n'avons pu nous empêcher d'en faire la comparaison avec celle qui est employée dans l'île de Syra pour guérir la jaunisse. Voici le résultat de nos réflexions et des"découvertes que nous avons pu faire.

M. d'Or, fatigué cles maux de poitrine, d'un crachement de sang, et d'une extinction de voix, épuisa inutilement toutes les ressources de la médecine, pour se procurer quelque soulagement. Dans ses recherches, il fut assez heureux pour trouver l'eau merveilleuse dont nous venons de parler. $\mathrm{M}$. d'Or, après trente-cinq ans de manipulation, l'a tellement perfectionnée, qu'elle ne laisse plus rien à désirer, s'il faut l'en croire (1).

(1) J'ai souvent entendu dire à M. d'Or que, dans les commencemens, son eau n'avoit pas toute la perfec- 
SUR L'île de SYr'A. Chap. XII. azi

11 prétend que, par son opération, il attire ct fixe dans l'eau la substance universelle qui vivifie toute chose, et qui se trouve dans les quatre élémens, sur-tout dans celui de l'air; et c'est cette substance qui domne à l'eau d'or l'admirable propriété d'être utile pour toutes les maladies, et souveraine dans la majeure partie: il lui a plu de l'appeler canastrale.

La manipulation de M. d'Or, m'est absolument inconnue; tout ce que je peux dire, c'est qu'il expose de l'eau à l'air, et tout ce qu'il fait, ¿l le fait sans feu, la nuit, vis-à-vis l'horison, et qu'il choisit pour cela un temps propice, qui es

tion dont elle jouit actuellement. Voici comment il s'explique là-dessus : dans l'air, à cause des vapeurs que la terre exhale continuellement, il y a quantité de matières hétérogènes, et même nuisibles et envenimées: ces matières, répandues dans l'air, sont attirées et fixées arec la substance universelle, par le procédé de M. d'Or. Moins cette substance est altérée par ces matières, pluselle est parfaite et salutaire; il est donc question de. l'en dégager quand elles s'y sont unies : c'est ce que $M$. d'Or a obtenu par une seconde manipulation, que nous ne connoissons pas, par laquelle il a tellement purifié la première substance, qu'elle peut produire beaucoup de bien sans le danger du moindre inconvénient. 
le printemps sur-tout, et lorsque les vents de nord soufflent.

Sessuccès ayant été complets dans le Languedoc, sur-tout à Uzès, à Alais, à Nîmes et à Montpellier, il passa ensuite à Rochefort, et enfin dans la capitale du royaume, où ayant reconnu que son cau n'avoit point autant de force que dans les provinces méridionales il a cru devoir l'exposer plus long-temps aux influences de l'air. En conséquence, il l'avoit laissée pendant quelques jours prendre plus de consistance. C'est dans cet intervalle qu'il s'est aperçu, au fond du vase, de la crystallisation d'un sel inconnu, qu'il a appelé aćrien, et d'après plusieurs expériences, il s'est convaincu que c'étoit dans ce sel que consistoit toute la vertu de son eau. Quoique cette espèce de sel fût invisible quand il opéroit en Languedoc, parce qu'il n'avoit pas le temps de se congeler ou de se crystalliser, il n'en est pas moins vrai que ce r'étoit que par son efficacité que son eau agissoit si heureuscment sur les maladies.

Nous avous été amenés naturcllement, du récit qu'on nous a lait du procédé de $\mathrm{M}$. d'Or dans la composition de son eau, à celui de 
SUR L'île de Syra. Chat. XII. 273 Syra, pour la guérison de la jaunisse. Ils paroissent fondés l'un et l'autre sur la même base. Chez nous, c'est une pièce d'or qui en fait la partie essentielle; dans l'eau d'or, c'est un ingrédient dont nous n'avons pas la connoissance; mais l'analogie cesse par la crystallisation des sels que nous n'apercevons pas dans notre procédé, et qui remplit merveilleusement le grand objet de la cure des maladies les plus aiguës. L'eau d'or agit sur elles arec violence et célérité, elle met en peu de temps le malade hors de danger. L'eau de Syra lui ressemble encore en cela, puisqu'en trois ou quatre jours elle emporte une jaunisse invétérée.

Dans les maladies chroniques, l'eau d'or agit avec lenteur; le mal ayant, pour ainsi clire, gangrené le sang et les os, il faut du temps pour que sa vertu médicinale puisse s'insinuer dansles parties les plus secrètes. C’est principalement sur l'estomac et la poitrine qu'elle opère avec plus d'activité et de vitesse. Comme ces parties du corps humain en reçoivent les premières impressions, elles doivent en ressentir les premiers bons effets. Sans faire aucune observation sur les qualités du vent, lorsqu'il s'agit de composer notre eau, nous faisons attention 
seulement que le temps ne soit pas à la pluie. M. d'Or n'expose pas la sienne entierement au serein; il la tient dans des endroits couvèrts, maispercés du côté du Nord, de manière que l'air puisse librement entrer et sortir. Il a remarqué que lorsque ce vent règne, et que le ciel est pur et serein, les sels aériens s'y multiplient à vue d'œil. Au surplus, il nous a dit avoir remarqué plusieurs fois dans ses vases une espèce d'huile qui surnageoit à la surface, quand il faisoit l'extraction de ces sels aëriens.

Malgré la certitude que j'avois des bons effets de ces deux procédés, ce n’étoit pas assez pour me tranquilliser, ni pour me défendre contre les attaques et les railleries de ces esprits qui trouvent plus facile de tout condamner, que d'observer et de s'instruire. Je désirois sur-tout de m'assurer si ces procédés pouvoient s'appuyer sur l'art de la médecine, ou sur les découvertes de la chimie; je m’appliquai en conséquence à l'étude de quelques auteurs sur ces matières, et jai trouvé, à ce qu'il me semble, et dans les princjpes de l'art de guérir, et dans ceux de plusieurs chimistes, tout ce qu'il falloit à un homme

raisonnable 
SUR L'íle DE Syr.a. Chap. XII. 27.5 raisonnable pour établir la justesse de ces deux procéclés.

Les auteurs dont j'ai tiré le pius de lnmières sur l'objet de cette discussion, sont M. le Clerc, ancien Médecin des Arnées du Rui en Allcmagne, daus son Hisioire nalurclle de l'Hoimme malade, et M. Sancassani, autcur italien, dans son Ouvrage intitulé, L'Anatomie de l'Eau.

En parlant de l'air, voici ce que M. le Clerc dit dans son ouvrage, que nous avons cilé tom. II, pag. 306 : «Que conclure de ces expériences? "Qu'il existe dans l'air une certaine vertu qu'on "ne peut déduire de toutes les propriétés que " nous lui connoissons. Sendivogius a soutenu "que l'air contenoit l'aliment secret de la vie. "Quelques chimistes ont dit la même chose; " mais qu'est-ce que cet aliment? comment agit" il? quel est sont effet réel? C'est ce qui nous est "parfaitement inconnu : heureux celui qui dé" couvrira ces choses!... »

Voicice que nous pensons de l'essence de cet aliment secret de la vie, de la manière dont il agit; et de ses effets sur les corps.

Il existe dans tous les végétaux deux principes de vie, sur lesquels, comme sur deux. pivots, roule leur conservation : c'est la chaleur Tome $I$. 
275 PRÉCIS HISTORIQUE

vitale, et l'humide radical. L'air les contient tous les deux, et je le trouve confirmé par l'expérience d'un chimiste célèbre, dans une brochure intitulée : Historia pracipuonum experimentortu circa analysim chimicam aëris atmospherici, nuàque principionum ejus in componendis diversis nalure corporibus. Aulore Freder. Lud. Schurer. Argentorati, an. 1789. Dans la préface de cette brochure, on lit ce qui suit : "Tllustrissimus Lavoisier, "naturam aeris venalus non lantim propriis, "nec ex alieno fonte hanslis periculis aeris "compositionem, ei fluidorum illam compo"nentinm rationem perspexit, verimetiam "ipsam illam auram, qua soli vita hominum "animantiumque presest, exconjunclione dua" rummateriarum nasci docuit, quanum nna, "si libera vinculisque suis soluta est, caloren "percoipora diffundit; altera antem commune "respirabilisaeris, aqua, acidorum et calcium "quas vocant metallicanum, principium effi« cit. »C'est-à-dire: « M. Lavoisier s’étant appliqué à l'étude de la nature de l'air, a rëconnu qu'il étoit un corps composé, ainsi que les fluides dont il est formé, et cela par des expériences que personne arant lui n'aroit entre- 
SUR L'íle de Srra. Chä́. NII. 277 prises. Il nous a appris aussi que ce principe, qui seul préside à la vie de l'homme et des animaux, provient de l'union de deux matières, dont l'une, si elle est libre et dégagyée de tous ses liens, répand la chaleur dans les corps; l'autre produit ensuite le principe qui est commun à l'air que nous respirons, à l'eau, aux acides, et aux chaux appelécs métalliques. »

Il semble donc très-probable que le principe secret de la vie, existant dans l'air, n'est autre chose que cette matière qui répand la chaleur dans les corps, caloren per corpora diffundit, et qui opère lc principe de l'eau, principium aquacfficil, Il suit delà, $I^{\circ}$. que ces principes ne doiventagir qu'en fomentant et la chaleur vitale, et l'humide radical; $2^{\circ}$. que leur effet naturel est de conserver les végétaux, en résistant à tout ce qui pourroit leur nuire, et en leur domnant assez de force pour repousser le mal quiles auroit gagnés.

En supposant la solidité de cette observation, i) s'ensuivroit que, comme la nature a une marche uniforme et constante dans la formation et la destruction des êtres, elle doit en avoir une constante et uniforme clans leur réjaration. 
Eh! qui sait si ce n'est pas M. d'Or qui a trouvé le moyen de fixer ces principes aériens qui contiennent l'aliment secret de la vie? Qui sait si cette matière, qui se cristallise au fond de ses vases, n'est pas cet aliment? Qui sait enfin si le vase d'ean, dans lequel on a mis une pièce d'or, et qu'on expose au serein, n'a pas une vertu proprè̀ attirer de l'air de ce même aliment?

Mais suivon' M. le Clerc, qui, page 552, parle ainsisur le même sujet: "L'existence d'un acide * universel, dit-il, est prouvée; des entrailles " de la terre il s'élève dans les régions supé"rieures, et chaque particule de l'air en con" tient. Seroit-il le vrai principe sans lequel les "animaux et les végétaux ne peuvent subsis"ter? Je le crois; et je pense que l'air cesse "d'être élastique, dès qu’il perd son aci-. "dité. "

S'il est vrai que cet acide est le seul principe qui fait subsister tous les végétaux, ne seroit -il pas possible que $M$. d'Or eût trouvé le moyen de le fixer, et de le rendre utile au genre humain? On seroit d'autant plus tenté de le croire, que l'eau pure de $\mathbf{M}$. d'Or porte avec elle une espèce d'acidité.

"On a observé, poursuit M. le Clerc, que cet 
sur lîile de Syra. Chap. XII.

acide abonde dans l'air, lorsque les vents d'Orient et du Nord soufflent, et que le temps est serein. Ceux qui travaillent aux mines remarquent que c'est particulierement lorsque ces vents règnent que leur terre alhaline simprègne d'un acide. L'acide nitreux r'est point différent de celui de l'air qui cause les éclairs; car la terre alhaline, qui est la base du nitre, est neutralisée par l'acide de l'air, toujours présent; il est si fortement altiré par les sels dalkali de toute espèce qu'on y expose, quà force d'en être imprégné, il devient tout-à-fait neutre. Alors l'acide aérien déguisé et caché sous le sel alkali et l'hule arec lesquels il est uni, produit un dissolvant quion a nommé admirable, d'après ses effets dans l'obstruction. »

En lisant tout ce que M. le Clere rapporte de ce sel neutre, on seroit presque tenté de croire quil a voulu nous donner le procédé de $M$. d'Or; en eliet, ce clemier assure que les sels aériens se fixent abondamment, lorsque le temps est serein, et que les vents sont au Nord ou au Levant. Nous arons déjà fait remarquer qu'il aroit plusieurs fois observé qu'me espèce d'huile sumagcoit dans ses rases; que les sels qui se déposoient au fond de ces mêmes vases étoient 
280 Piŕtis HISTORIQUE

d'une nature différente de tous ceux que lion connoît, et quils paroissoient être nentres; enfin, si toutes les maladies, comme le préten. dent différens auteurs, proviennent des obstructions qui se forment dans plusieurs parties du corps, et si le sel neutre, dont parle M. le Clere, cst cfficace pour ces espéces de maux, powrquoi ces sels ne seroient-ils pas du plus grand secours dans les autres maladics? L'eau d'or, ćtant composće arec des sels très-analogues à ceux de $\mathbf{M}$. le Clere, doit être infiniment utile dans beaucoup de circonstances; et le procédé de son anteur, aninsi que celui que nons pratiquons au Levant, bien loin de s'opposer anx principes de la médecine, Jui est an contraire conforme.

Niais il fant cxaminer si l'on trouve aitlenis des autorités qui puissent confirmer ce que nons arons avancé.

D'abord le docteur Sancassani uousen a fourni un grand nombre, et javone que jai ćté étonné de la ciarté qu'elles répandent sur le procédé de MI. d'Or. C'est dans un petit onvrage que ce médecin rélèbre a publié sur l’anatomic de l'cau, que lon tronve véritablement des vues profondes sur notre théoric. Les différentes opi- 
SUR L'íle De Syra. Chap。XII. 28 i nions des anciens, sur la substance de l'eau, exciterent l'auteur à s'appliquer sérieusement à cette analyse, et pour le faire avec succès, il n’épargna ni son temps ni son arģent : il s'étoit associé plusicurs persomes de l'art pour assister à ses expériences; leur résultat fut toujours, que l'eau renfernée hermétiquenent dans des vases de verre, poussée à l'extérieur par un feu modéré qui excitoit celui qui étoit caché au centre de l'eau, produisuit deux vapeurs, l'une blanche et l'autre ronge, qui s'attachoient avec le temps et par parties aux parois des rases. Ces vapeurs se convertissant bientôt en cau, il en sortoit un es. prit subtil et invisible, qui aroit beaucoup réchaufé lesvases (1), ct que je ponrrois appelè, dit

(i) Par tout ce que Sarcassani dit ici, et par ce que nous rapporterons de lui plus bas, on découvre une grande analogie entre les opérations de ce chimistesur la nature de l'eau, et celles de MI. Lavoisier sur la substance del'air. Les résul tats des unes et des autres se rapprochent; et on peut conclure que daus l'eau, de même que dans l'air, est contenal'aliment secret de lavie, ou ccte substance unirerselle qui virifie tous les végétaux. Effectivement cet esprit subtil et invisible, qui réchaufe les rases, et qui s'agite arec impétuosité, ne paroît être autre chose que ce même principe, découvert dans l'air var 
882 PRÉCIS HISTORIQUE

notre auteur, de l'air agité, ou du vent, à canse de son impétuosité. Ensuite, les parties grossières de ces vapeurs se précipitoient an fond du vase sous différentes formes, tantôt en terre crisıalline, tantôt en forme d'écailles de poisson, et quelquefois en espèce de talc.

Enfin, l'eau se clarifioit quand elle étoit dégagée de ces vapeurs et de cette terre. Les sels se produisoient, et l'on royoit surnager de petites gouttes semblables à des grains de millet : ces gouttes avoient l'apparence de Thuile (1), brûloient, et donneient de la flamme comme les autres matières olćagineuses.

Maisc'est dans l'onvrage de ce savant médecin qu'il faut voir tous les moyens dont il s'est servi, pour parvenir à de si grandes découvertes. Pour ce qui nous regarde, nous devons nous en tenir à ce qüil a dic des subsiances spiritueuses, terreuseset salises que l'on extrait de son eau : les bons

M. Lavoisier, qui calorem per corpora diffundit. Cette substance, qui se fait voirensuitesous la figure d'écailles ou de tale, n'est peut-être autre chose que l'essence de l'humidité de l'eau même.

(I) M. d'Or m'a plusieurs fois assuré avoir souvent obscrvé de petites gouttes, comme d'hnile, surnager dans les vases d'eau qu'il exposøit à l'air, selon son procédé. 
SUR L'île DE Syra. CHA A. XII. 283 effets qu'elles produisent'sur l'homme malade, sont uniquement de notre sujet.

"Venons, dit l'auteur, aux remédes qu'on a découverts dans l'eau, dont jusqu'à présent on a fait différentes épreuves dans la médecine. En premier lieu, il y a l'esprit, qu'on retire de l'eau de pluie et de neige, et qui, comme je l'ai remarqué, échaufie les vases. Cet esprit rectifié, étant très-simplé et très-subtil, a par lui-même une grande énergie: lorsqu'on l'applique aux fières continues, intermittentes, ardentes, il coupe le levain fébrile, dissout les présures sans violence, rafraîchit; et peut être employé en tout tempset à tout âge, sans danger. Il provoque les urines et la sucur, selon les dispositions des malades; j̣l dégage les obstructions, et parvient jusqu'à la quatrième digestion, fortifiant avec efficacité les esprits animaux pour expulser la cause des maladies; et si l'ean naturelle commune, qui est pleine d'impuretés, gućrit tontes les plaies d'après la méthode de Filippo Palazio (1), avec combien plus de promptitude et de facilité ne doiventelles pas se ğuérir, arec cet esprit d'eau, yni

(1) Je connois une personne qui, s'étant donné un grand coup de hache aupied en fendant du bois, a été guérie avec la seule eau d'or. 
résiste à la corruption et qui anime davantage. les esprits pour se joindre et se glutiner? Si ensuite on dissout dans cet esprit d'eau, les substances balsamiques, et propres à la guérison des plaies, ii sert pour les ulceres : nous en avons vu des effets admirables dans un très-grand nombre de cures opérées par ce moyen. »

"Avec ce même esprit on prépare des ext raits, des sels, des magistères, des essences, des baumes; et en y faisant dissoudre des gommes, on en retire des teintures vulnéraires, et on en fait plusieurs autres préparations. Un grand avantage sur-tont, c'est qu'il est très simple qu'il n'altère point les choses, et ne change point lcur vertu naturelle. Ajoutez encore que toutes ces compositions, on mélanges, peu. vent se faire sans feu, (I) comme la gomme de tartre qui se prépare en la broyant à la molette et en la dissolvant dans cet esprit; on l'expose ensuite au soleil pour évaporer son humidicé, jusqu’à réduction en consistance du miel. »

"De plus, on peut tirer de l'eau une terre de plusicurs espèces. La meilleure est la plurs luisante; clle ressemble aux écailles de poisson, ou bien à du talc. Cette terre est appelće

(2) I'cau dic M. d'Or se fait sans feu. 
sur l'île de Syr a. Char. XII. 285 par les philosophes, terre philosephique, terre vierge. Elle sert à plusieurs cures, particulièrement pour les maux de poitrine, parce qu'elle absorbe lesacides corrosifs. Par la même raison, clle gunérit de la dyssenterie, des tranchées, etc.; elle est bonne pour les plaiesphagédéniques et corrosives, et pour certains ulcères regardés comme iucurables; elle adoucit le sang, et produit enfin lesplus admirables efiets : sa force et sa vertu pour la guérison des maladies les plus graves, pourroient bien s'attribuer à la qualité qu'elle a de contenir une teinture de couleur d'or volatile. »

"Quoi qu'il en soit, on retire de l'eau unc espéce de sel qui d'abord ressemble au sel commun, mais qui à la longue devient âpre et amer; il finit cependant par être trés-loux et très-froid, quand on l'applique sur la langue( 1 ). Cesqualités le rendent très-utile dans des fièvres ardentes et malignes: comme il est pénétrant et réfrigćrant, il résiste aux miasmes empoisonneurs et aux levains fébriles; il provoque les sueurs, et les urines, et il calme les grandes ardeurs et la

(1) Les scls de M. d'Or, ou sun élixir pur, appliqués sur la langue, scmblent avoir toutes les qualités de ce scl tiré de l'eau. 
sécheresse qui accompagnent toujours les fièves violentes. Avec ce sel Oliverius de Oliveriis aiguise son menstrue pour dissoudre l'or, et poirr le rendre potable. Cet or étant préparé avec ce sel, comme l'auteur nous l'apprend, produit les effets les plus merveilleux."

"Ce qui doit sur-tout étonner, c'est de voir combien ilsc multiplic, lorsqu'on l'expose à l'air. J'ai souvent observé que, lorsqu'il étoit clans un vase fendu, il s'échappoit par toutes les fentes; subtil et actif, il s'y attachoit, il s'y incrustoit (I). En été, au milieu des plus grandes chalcurs, il lui faut toujours de l'ombre (2), pour se

(1) J'ai observé moi-même, autour de l'orifice des flacons qui contenoient l'essence d'eau d'or, ( $j$ 'appelle ainsi ectte premiere matière d'un goût acide, dont on met dans une pinte d'eau de riviere, la quantité de deux ou trois dés à coudre ); j’ai vu, dis-je, cet orifice couvert d'ume espèce de sel, ayant le même goût que l'essence. En observant ces sels au microscope, ils ont une forme ronde „ou tubeuse. On ignore s'ils sont produits parunsuintement de la matière autour du bonton du flacon, ou sils sont des sels aériens que la vertu attractive de la matière contenue dans le flacon, y attire et y fixe.

(2) Nous avons observé que le procédé de guérir la jaunisse, et celui de M. d'Or, s'opèrent dans l'ombre, et dans les belles journées. 
SUR L'île de Syra. Chap. XII. 287 former eil plus grande quantité. Enfin, les sels contiennent des vertus admirables, ce qui a fait dire à certains philosoplues, in sole saleque omnia; et à d'autres, in solo sale omnia."

"Mais rien n'est comparable à la seconde terre qui se produit après la dissolution de la première, en sa coaggulant une seconde fois. La première terre, qui d'elle-même se change cn une eaugrasse, est celle que Sendevogius appelle mercure philosophique. La seconde ressemble à une gromme pendante, tirant sur la couleur de chair; elle se dissout dans l'eau qu'elle fait venir comme du lait: employée dans les maladies et dans toute espèce de fièvres, elle opère des prodiges. "

"Quant à l'esprit qu'on retire de l'eau, on peut dire qu'il surpasse tous les autres avantages; par son moyen on dissout l'or, et on le rend potable; on dissout le fer, et l'on en retire une teinture souveraine pour les dyssenteries; du crayon, on en fait une qui guérit le flux desang; de l'étain, qui anéantit les effets utérins; et de l'argent, qui délivre d'un grand nombre de maux: enfin, on obtient avec cet esprit toutes sortes de remèdes, par le moyen des minéraux et de métaux; et je suis d'avis que c'est l'esprit du 
mercure que Basileo Valentino vante hautement dans son traité de rebus naturalibus, et sans lequel il affirme qu'on ne peut taire riea de bon et de grand.

C'est avec cet esprit qu'on prépare des remèdes bien plus efficaces qu'avec l'alkaest: sa qualité dissolvante ôte non-seulement la corporéité aux choses; mais, comme ils est trèsactif, il sunit inséparablement avec celles qu'il a dissoutes, et il donne à leur vertu un degré de perfection qu'elles n'avoient pas. "

"Par tout ceque nous avons dit, conclut Sancassani, il paroît certain, d'après l'anatomie de l'eau, que si cette substance étoit purifiée et parfaitement réduite, et qu'elle ne pût recevoir ni changement, ni altération quelconque, elle pourroit passer à juste titre pour ce que les philosophes ont appelé la médecine universelle. "

M. d'Or prétend que son élixir, ou la substance quil verse dans une pinte d'eau, est tellement purifiée et réduite, qu'elle ne peut recevoir ni changement ni altération quelconque; 'le sorte qu'elle conserve toujours sa vertu médicinale, même après l'avoir fait passer par le feu. 
sur l'île de Syra. Chap. XiI. 289

Conclusion. Ou M. d'Or travaille effectivement, comme ille dit à qui veut l'entendre, avec de l'eau et de l'air, et il en tire véritablement les principes vitaux qu'ils contiennent, ou tout ce qu'il dit sur son travail, n'est autre chose que quelque procédé semblable à ceux de Sancassani, pour tirer de l'eau son esprit, ou le sel et la terre qu'elle contient, Quoi qu'il en soit, qu'il manipule l'eau par les moyens exposés ci-clessus, ou d'une autre manière, il n'est pas moins vrai que son procédé est fondé sur des principes connus dans la chimie, et avoués par l'art de la médecine, et que le nôtre s'appuie sur des bases semblables, quand nous gućrissons la jaunisse avec de l'eau, exposée au serein, dans laquelle on a mis seulement une pièce d'or. Il est de fait, et je le repète, qu'il n'y a pas un seul exemple à Syra d'une personne qui n'ait été guérie en trois ou quatre jours par ce remède aussi simple que facile. Il est de fait aussi, qu'avec l'eau de M. d'Or, on peut attaquer victorieusement les maladies les plus opiniâtres, et surtout la pulmonie, à laquelle rien n'a pu être aussi efficacement opposé jusqu’ici. Les épreuves qui en ont été faites à Míntpellier, à Nîmes , à Rochefort et ailleurs, et sur-tout dans la ca- 
pitale, en sont des preuves incontestables. On peut tous les jours, et à tous les momens, les vérifier.

Ajoutons enfin, que quantité de personnes regardent l'eau d'or comme un remède de charlatan, sous le seul prétexte que son auteur lui donne un air de remède universel. Cela ne fait rien contre mon opinion, et je suis persuadé que jusqu'à présent on n'a point trouvé une médecine, que l'on puisse appeler effectivement mniverselle; mais prétendre qu'elle n'existe point dans toute la nature, c'est ce que je suis très-loin decroire. Nous avons observé déja que la nature, ayaut une marche uniforme et constante dans la formation et la destruction desêtres, devoit en avoir aussi une constante et uniforme dans leur réparation. Or, d'après les principes constitutifs que l'on suppose aussi à l'eau, il suit que l'idée d'un remède universel ne lui est pas contraire. 


\section{T R A I T E}

C O M P L E T

SUR LES ABEILLES.

\section{I V R E I,}

Contenant tout ce qui regarde la culture des abeilles en général.

C H A P I T R E I.

Des pays les plus propres à élever des abeilles, de la qunutite de ruches que chaque pays peut nourrir, et de la siluation la plus avantageuse qu'il convient de leur donner.

Ex général, tous les pays de l'univers, qui produisent des fieurs, sont propres à la cultare dés abeilles; mais les contrées les plus méridionaies, ou celles d'un climat tempéré, leur sont lien

Tome I. 
plus favorables qué les pays froids et septentrionaux. A l'égard de la quantité, et sur-tout de la qualité du miel et de la cire, ce n'est qu'au midi qu'on peut espérer de réunir ces deux avantages.

Aussi, dans tous les temps, les pays méridionaux, sur-tout la Grèce et l'Italie, ont été célébrés par tous les agriculteurs, pour la bonté et l'abondance de leur miel et de leur cire.

En général, les pays montueux produisent de meilleurs fruits que les pays plats, parce qu'ils sont plus secs; et c'est ce qui y rend aussi le miel et la cire d'une meilleure qualité.

Quoique Pline prétende que le miel de l'île de Corse soit amer et d'un goût désagréable, il n'en résulte rien contre ce que nous venons d'avancer en faveur des pays méridionaux. Nous pensons comme Diodore de Sicile et autres, que cette qualité défectueuse n'a d'autre cause que le voisinage clu buis et de l'if, dont les fleurs sont trèspréjudiciables au miel. On lit, dans Virgile, que Licidas ne vouloit pas que ses abeilles se nourrissent avec le suc de l'if de Corse.

Sic tua cyrnæas fugiant examina taxos. Eclug. $1 x$, ข. 30 .

Il arrive également dans l'Archipel, qu'après 
SUR les abeilles. Liv. I. Chap. I. $29 \mathrm{I}$ la récolte et la vendange des ruches, les abeilles tirent d'une certaine plante (dont la fleur s'appelle dans l'ile de Syra Iurcaki, petit Turc, et l'oignon, qui est un des plus gros qui existent, askella, scille en françois), une espèce de miel qui a un goût amer. Nous le laissons ordinairement en entier pour la provision des abeilles, ou si nous en retirons quelque petite partie, elle sert à soutenir les plus foibles pendant l'hiver. Malgré cela, le miel des îles de l'Archipel, celui de l'île de Syra sur.tout, ne laisse pas d'être un des meilleurs du Levant, et il n'en est aucun qui lui soit supérieur pour le parfum. De même la Corse produit assez généralement d'exccllent miel, quoiqu'il soit possible que, dans quelques cantons de cette île, où le buis et l'if abondent, leurs fleurs lui donnent un goût désagréable.

Quant à ce que nous avons dit des pays méridionaux, pour la quantité et la qualité de leur miel et de leur cire, il suffit, pour s'en convaincre, de lire ce que rapporte $M$. Mentelle sur la prodigieuse multiplication des abeilles qui furent apportées, il y a peu d'années, dans l'île de Cuba, l'une des principales des Antilles, située sous la zone torricle. Cet 
auteur (dont je ferai mention dans le troisième chapitre) parle de la quantitéincroyable de cire qui sort de cette île, et que produisent les ruches, en quatre récoltes qu'elles donnent à leurs propriétaires, chaque année. On ne voit certainement pas une semblable fertilité dans aucun des pays septentrionaux.

A l'égard de la quantité de ruches que pent entretenir un pays, nous ne sommes point du tout de l'avis de M. Lagrenée (que nous regardons comme un des hommes les plus instruits sur l'économie des abeilles), quand il dit que chaque canton peut en entretenir une quantité quelconque, sans quil en arrive aucun inconvéuient.

Pour faire mieux comprendre l'idée de l'auteur que nous venons de citer, et notre opinion particulière, nous rapporterons ses propres paroles, en y joignant quelques réflexions. Il commence ainsi le premier chapitre de son Traité sur les Abeilles, imprimé à Paris en 1783. «Je ne suis * pas du nombre de ceux qui pensent pouvoir cal* culer la quantité de ruches que peut nourrir * un pays; qui n'en donnent que cent à l'un, * pendant qu'ils en accordent cinq cents à un * autre de pareille étendue."

Nous pensons, cornme l'auteur, qu'il n'est 
SUR les ABEILles. Lir. I. Chas. I. 293 pas possible de déterminer d'une manière rertaine, la quantité de ruches que tel ou tel pays peut ou ne peut pas entretenir : au moins ne le pourroit - on pas avec la même précision qu'on établiroit celle d'un terrain qui suffiroit on ne suffiroit pas à nourrir un certain nombre d'hommes.

Cependant, comme il y a des terrains qui, étant infiniment plus fertiles les uns que les autres, peuvent par conséquent nourrir un plus ou moins grand nombre d'hommes, il y a aussi des pays et des cantons qui, quoique situés sous un même climat, sont infiniment plus fertiles en miel et en cire, soit par l'abondance, soit par la qualité des fleurs qui y naissent; d'vù il suit yutue ces cantons sont plus ou moins favorab?es à la multiplication des abeilles, et que les uns peuvent fournir aisément à quantité de ruches, pendant que les autres peuvent à peine en nourrir la moitić, et quelquefois moins encore.

*Quelquequantité qu'il y en ait, continue M. "Lagrenée, dans un pays, comme elle est "certainement proportionnée au nombre de « gens qui l'habitent, et à la culture qu'ils en "font, elles y trouvent suffisamment de miel * et de matière à cire à récolter. " 
A l'égard de cette assertion, nous ne saurions l'approuver, parce qu'elle est contraire d̀ l'expérience. La nature est limitée dans toutes ses productions, et chaque chose a un terme ; par conséquent, si, dans une île qui ne peut nourrir, par exemple, que dix mille personnes, vous $\mathrm{y}$ en mettez quinze mille, la disette s'y fera bientôt sentir; et si vous $y$ en mettez vingt mille, il en résultera la mort et la destruction de tous les habitans, en supposant que, dans cestrois hypothèses, la fertilité soit la même.

De même, si daus une étendue de terrain qui ne produit qưune quantité donnée de miel et d'autres provisions nécessaires pour l'entretien des abeilles (car il faut certainement en supposer une quantité déterminée; et comme nous l'avons déja dit, la nature est limitée en tout); si donc vous y mettez un nombre de ruches proportionné à une telle fertilité, de cent, par exemple;elles pourront facilement se pourvoir du nécessaire, et fournir une bonne q 'antité de miel et de cire à leurs propriétaires; mais si vous doublez ce nombre, elles auront à peine de quoi se nourrir, et par conséquent elles ne fourniront presque rien à leur maître. Ajoutez que sị 
SUR les abeilles. Liv. I. Chap. I. 295 l'année est tant soit peu mauvaise, ces ruches ne sauroient se conserver. Enfin si vous mettez, dàns un espace de terrain, plus du double d'abeilles qu'il n'est en état d'en nourrir, alors ne pouvant, comme nous l'avons dit, se pourvoir de ce qu'il leur faut pour la quantité excédente, elles périront nécessairement, ¿̀ l'exception peutêtre de quelques ruches, dont les abeilles par leur nombre et leur activité supérieure, auront pourvu mieux que les foibles, à leur subsistance.

Règle certaine : quand il y a peu de ruches dans un pays, ou qu'il n'y a que celles qu'il peut entretenir commodément, les abeilles tromvent leur nourriture, sans se donner beaucoup de peine, parce qu'elles peuvent faire dans la journée, jusqu’à vingt ou trente voyages. Mais quand les ruches sont en trop grand nombre, ct qu'elles excèdent la fertilité ordinaire et naturelle d'un pays, les abeilles ont alors beaucoup de difficulté à se pourvoir du nécessaire : elles sont obligées d'entreprendre des courses plus longues, et par conséquent elles font moins de voyages, et rapportent moins de provisions dans leurs ruches.

M. Lagrenée suppose que, "dans les pays qui $\mathrm{T}$ iv 
" ne leur sont pas d'ailleurs des plus favora. "bles, et qui néanmoins sont bien garnis d'a"beilles, il reste encore beaucoup de miel et " de matiere de cire qui se perdent chaquean"nće sur les feuilles et les fleurs, faute l'être "enlevés."

Cela peut être, mais c'est par la négyligence de quelques abeilles, qui, rebutées par les difficultés qu'elles éprouvent àse pourvoir de leur nécessaire, ne se donnent pas la peine d'aller chercher plus loin leur nourriture, on faute de commodité et de beau temps, ou enfin parce que ce miel et cette cire ont échappé à leurs recherches. On ne pourra jamais en conclure que tont pays peut nourrir un nombre illimité de ruches, et qu'une trops grande quantité ne puisse nuire au succès de cette culture.

M. Lagrenće ajoute encore, pour venir à l'appui de son assertion, que dans les pays vì les ruches se sont beaucoup multipliées, on n'a observé aucune diminution dans leur produit. Je réponds que cela n’a pu arriver que parce que la quantité de ruches qui existoit auparavant dans le pays, n'étoit point proportionnée à son étendue et à sa fertilitć naturelle en miel et en cire; car si le numbre des ruches 
sur les abeilles. Liv. I. Chap. I. 297 excéde la juste proportion qui doit exister, comme nous le répétons encore, il est clair que cette quantité excessive de ruches doit nuire nécessairement à la prospérité des abeilles.

On éprouve journellement dans l'Archipel, que lorsqu'il y a trop de ruches cians un même licu, ellesne réussissent pas aussi bien quecelles qui sont ailleurs en plus petit nombre : ces ruches ne produisent pas autant de miel et de cire. Nous regardous ceci comme une chose trèscertaine. En effet, c'est un fait avéré et constant dans l'île de Syra, que les ruclies qui se trouvent à l'extrémité de l'île, sont celles qui donnent une plus grande quantité de miel et de cire, parce que ces parties sont toujours les moins peuplées en abeilles. An contraire celles de l'intérieur de l'ile où ces abeilles abondent, ne sont pas aussi riches en provisions de miel et de cire, quoiqu'clles prorluisent plus souvent des essaims, et quelles virent plus long-temps, comme nous le dirons au neuvième chapitre du cinquiome livre.

M. Lagrenée conclut enfin "que quoique, "sans contredit, ily ait des pays plus propres "les uns que les autres à nourrir les abcilles, " on peut réammoins en aroir dilis toutes sortes 
"de lieux, et que personne ne doit faire dif"ficulté de s'en procurer, quels que soient le "terrain et les productions du lieu qu'on ha"bite. "

Nous sommes entièrement de cet avis; nous ajouterons seulement que lorsque l'onveut entreprendre la culture des abeilles, on doit toujours préférer, pour établir ces ruches, l'en. droit aux environs duquel il y en a le moins.

Venons actuellement aux positions les plus avantageuses pour cette culture. M. Palteau, cité par M. Ducarne, dans son quatrième entret., p. 68, distingue trois sortes de positions dans l'économie des abeilles, l'une médiocre, l'autre bonne, et la troisième excellente. "Je pense, dit cet auteur, qu'on peut distin"guer trois positions différentes, qui donne"ront trois diffërens produits. Les plaines de «bled, les prairies, les petits ruisseaux, for"ment ce que jappelle la moyenne ou la mé" diocre position. L'abondance de bled et des "prés, la proximité des bois, des grandes fri"ches et des ruisseaux, forment la bonne po"sition. Le voisinagre des prairies, du sarrasin, « des bois, de grandes friches, et des montagnes «couvertes d'herbes odoriférantes, l'éloigne- 
sur les abeilles. Liv. I. Chap. I. 299 « ment des étangs et des rivières d'une certaine «largeur, font l'excellente position : celle-ci "vous rapportera deux fois plus que la pre" mière, et doublera sur la seconde.

"Quoique ces positions soient les meilleures, « il n'est pourtant guère d'endroits où on ne "puisse placer des abeilles avec avantage, mais "non en telle quantité qu’on le voudra." Voilà encore M. Palteau qui s'oppose au sentiment de M. Lagrenée que j’ai combatur. "Il faut * examiner la qualitédu pays dans lequel on se " trouve, proportionner le nombre des liabi" tans à la quantité de nourriture que peutfour"nir ce canton, et ne pas placer cent ruches "dans un lieu qui n'en peut contenir que cin"quante. »On voit par là que M. Palteau pense absolument comme nous, que puisque la nature est limitée, il doit y avoir toujours une juste proportion entre les consommateurs et le produit du pays. Nous verrons plus bas ce que dit à ce sujet M. Ducarne.

"Lesvastes et fécondes plaines de la Beauce, " de l'Ile-de-France et du Soissonnois, conti" nue M. Palteau, qui sont des greniers à bled "pour la France, mais qui ont peu de prai"ries arrosées par des ruisseaux, cessent, dans 
"bien des années, de fournir aux abeilles de " quoi faire des récoltes, long-temps avant que " les saisons qui les retiennent renfermées dans " leurs ruches, soient proches.

"On arrache dans ces provinces, ainsi que s dans une bonne partie de la Picardie, tout le " chaume des champs, et en même temps toutes "Ies herbes qui s'y trouvent. Dans ces pays, * lorsque l'été est sec, aprèsque les foins ont été "coupés, ou au moins lorsque les bleds sont " mûrs, tout est aride clans les campagnes; les "abeilles ont beau les parcourir, elles n'y trou"vent point, ou si peu de fleurs, qu’à peine "celles qui sont les plus heureuses, trouvent"elles de quoi se nomrrir hors de leur ruche. «Enfin dès le 2 r juillet, ces provinces sont, " par rapport aux abeilles, comme le sont les * autres dans le mois de septembre. Il faut donc " se régler sur la connoissance qu'on a du canton "qu'on habite. "

" M. Ducarne se plaint que M. Palteau est * trop général sur ce sujet, qu’il auroit dû en« trer davantage dans le particulier, et donner "quelques exemples pour y suppléer : je vous "dirai, ajoute-t-il, re que mon expérience * ma appris à ce sujet. Je dis donc que je crois 
sur i.es abeilles. Liv. I. Chat. I. 30 i " qu'on peut placer cent ruches au moins dans " un canton d'une position médiocre; deux " cents dans une position bonne, et quatre ou " cinq cents dans une position excellente. Au "reste, quoique dans les provinces, telles que "celles que nous venons de nommer, la récolte " du miel, e même quelquefois celle de la " cire, cesse dès le 25 ou 30 juillet; elles perr* vent néanmoins être encorc propres à y éle"ver des abeilles, et on peut y faire encore un "bénéfice honnête, quelquefois même consi* dérable, si la campagne est favorable; et "même, dans les meilleures positions, la ré" colte du miel re va gucere au delà du 5 ou 6 " d'août, et quelquefois elle finit dès le 20 « et même le 12 , ou le 15 de juillet.

"Ces positions n'ont done pas alors beaucoup « d'avantage sur les médiocres par rapport à l'a"bondance des fleurs; car il n'y a guère de " position', à moins qu'elle ne soit très-mauvaise, " où les flcurs manquent depuis les i 5 ou 20 " juillet, et jusqu'alors les abeilles en trou«vent toujours assez. Je ne parle point ici des * endroits où indépendamment des autres avan" tages d'une exccilente position, on auroit ce« lui d’y trouver encore des sarrasins ou blés 
«noirs, parce que cẹtte plante ne donne ordi"nairement sa fleur, qu'en aøût et en septem"bre, et que quoique dans cette saison, les au. " tresfleurs aient communément peu de saveur, " ou qu'aumoins elles contiennent peu de miel, " celle du bled noir est mieux fournie; mais * il est rare que toutes ces choses se rencon" trent. Enfin, je ne connois guère d'endroits " où on ne puisse élever des abeilles avec quel"que avantagge; les abeilles y fussent-t-elles " obligées d'aller butiner les fleurs à une lieue "de-là, le mâ̂tre n’y perdroit pas encore." Tel est le sentiment de M. Ducarne, qui est aussi un des cultivateurs les plus instruits sur les différentes combinaisons dont l'économie des abeilles cst susceptible.

A l'égard du bled sarrazin, nous ajouterons ici qu'une personne digne de foi, nous a assuréqu'en Franche-Comté, au moyen de ce bled, les ruches domnent quelquefois jusqu'à 30 et 40 lirres de miel : abondance prodigieuse qui, laisséc aux ruches, met les abeilles dans le cas de passer les plus longs hivers fort à leur aise; ce qui devroit porter tous les amateurs à étendre daus le royaume la culture de cette espèce de bled, ayec d'autant plus de confiance, qu'elle est très- 
SUR LES ABEILles. Liv. I. Chap. I. 303 facile, et qu'elle peut réussir dans toute sorte de terrains.

On ne connoît pas cette espèce de bled dans l'île de Syra; mais nous sommes certains que si on l'y introduisoit, ce seroit un avantage infini pour les abeilles. Il seroit d'autant plus précieux que dans cette île, ainsi que dans tout l'Archipel, où le climat est très-chaud, il arrive souvent qu'avant la fin de mai, il n'y a plus de fleurs dans la campagne pour la pâture des abeilles; et sans le secours du thym, dont nous parlerons ailleurs, qui vient vers le milieu de juin, et dure jusqu'à la fin d'août, les ruches seroient fort à plaindre pendant tout cet intervalle : enfin le sarrazin y fleuriroit beaucoup plus tôt que dans ces pays-ci. Ajoutez à cela, que le thym étant une plante très-clélicate, il est difficile qu'il réussisse tous les ans. Dans le cas donc où il manqueroit, il pourroit être remplacé par le sarrazin.

Si l'on peut en introduire la culture, il est certain qu'il en résultera un grand bien pour la prospérité et la conservation des abeilles, tant dans le Levant qu'en France, et sur-tout dans ses provinces méridionales, où les fleurs doivent manquer plus tôt que dans les autres. 
Enfin, avant de terminer ce chapitre, nous al. lons rapporter ici une autre observation de $\mathrm{M}$. Palteau, cité par M. Ducarne, entret. 4, pay. 64, relativement à la position des ruches. "Le voisi" nage des étangss et des grandes rivières est fort " pernicieux aux abeilles, parce qu’il y en périt « un trè-grand nombre, sur-tout dans le tems " des grands vents et des forts orages. Elles doi" vent être éloignées des grandes villes, où elles " s'introduisent assez volontiers chez les confi" seurs et chez tous ceux qui préparent les su" creries; mais elles paient fort cher leur gour" mandise et leur sensualité, parce qu'elles en" trent dans toutes sortes de pots et de vases, " et qu'elles y sont souvent noyées par l'eau * qu'on y jette.

"En les écartant des lieux où il y a du sucre " et du miel, il semble quon devroit bien plus "encore leur interdire les bourbiers, les fu"miers, et autres lieux inlects et puants : mais « non, on a observé que ces endroits ne leur sont "point désagréables. Elles recherchent, par « exemple, avec empressement, les eaux salćes, "les lieux imbibés et infectés d'urine, l'eau dé"trempéc dans la fiente de bœufs et les égoûts "des fumicrs. "Nousreviendronsaillcurs surces observatious.

CHAPITRE 
sur les abeilles. Liv. I. Chap. I. 305

\section{H A P I T R E II.}

Des arbres et des plantes don les fleurs servent i la nourriure des abeilles.

Quelques personnes regarderont peut-être comme inutile le clétail que nous nous proposons de donner de plusieurs arbres, plantes et fleurs qui fournissent plus de miel et de poussière, et qui paroissent être d'une grande utilité pour laculture des abeilles; mais nuus avons cru qu'il ne servit pas désagréable aux amateurs, et qu'il exciteroit la curiosité et l'attention de beaucoup de personnes industrienses, qui, zćlées pour le bien commun, pourroient procurer à-leur pays toutes les plantes propres à la nourriture cie ces insectes, ou au moins celles qui sont de nature à y réussir.

Au reste, nous ne proposons rien que nous ne soyons déterminésà exécuter nous mêmes en en partant pour le Levant. Nous comptons emporter des graines de certaines plantes qui n'y viennent pas, pour les propager dans les Tome $T$. 
ŝles de l'Archipel; afin d'enrichir le domaine de nos abeilles, et de faire ensorte qu'elles deviennent plus utiles à leurs propriétaires.

$\mathrm{Si}$ les plantes qui sont utiles anx abeilles dans les pays froids, pouvoient réussir dans les climats tempérés, nous ne doutons pas qu'elles ne leur fussent du plus grand avantage; car on sait que les mêmes theurs qui viennent également et dans les pays froids et dans les pays tempérés, produisent ordinairement dans ceuxci, une plus grande quantité de miel que dans les premiers.

Il y a de plus une autre utilité dans la connoissance des arbres et des plantes les plus propres aux abeilles; c'est que lorsqu'on est naître de l'emplacement des ruches, il faut toujours préférer celui où ces plantes règnent avec le plus d'abondance, jusçu’à une lieue à la ronde.

Nous avons tiré la liste de tous ces arbres, des notes de M. Contardi sur le Traité dẹ M. Wildman, concernant la manière de gouverner les abeilles. Nous y avons ajouté la manière abrégée de cultiver clracune de ces plantes, d'après le'Dictionnaire d'Agriculture de M.Ranconi, Florentin, que nous arons entremêlée de quel- 
Sur les abeilles. Liv. I. Chap. II. 307 ques-unes de nos observations. Par ce catalogue alségé, notre but est d'exciter la curiosité des amateurs. Les auteurs, (qui Dieu merci ne manquent pas en Europe), pourront faire une étude plus approfonclie de la propriété des fleur's, relativement au temps en particulier, et suivant le climat de chaque pays où elles se trouvent : ce sera le moyen d’avoír une succession continuelle de fleurs, propres à nourrir les abeilles depuis le mois de mars jusqu'au mois de novembre; et dans les lienx où la nature ne produiroit pas une pareille succession, l'art et l'industrie de l'homme pourront aisément y suppléer. M. Contardi devoit parler dans un nouvel ouvrage de cette succession artificielle de fleurs. Nous ignorons sil a été publié; mais nous pensons qu'il seroit très-avantagreux à la prospérité des abeilles, que quelque personne instruite s'appliquât à donner la nomenclature des fleurs les plus utiles à ces insectes, ayant égard, comme de raison, aux différens climats : il faudroit aussi qu'on y ajoutât la méthode de cultiver depuis le mois de mars jusqu'au mois de novembre, dans les campaznes et dans les jardins, toutes les plantes qui pourront leur fuurnir un aliment con. 
tinuel. M. Contardi fait précéder son catalogue dles plantes qu'il'croit les plus intéressantes pour la nourriture de nos insectes, par des observations, sur lesquelles nous nous permettrons quelques réflexions.

"Avant, dit-il, de parler des fleurs, on me "permettra de faire observer que nos abeilles " doivent avoir un goût plus grossier que les " autres, (M. Contardi parle, comme Italien, " des abeilles d'Italie, ) qu'il ne m'est jamais " arrivé de voir s'arrêter sur les fleurs de la "mélisse ou citronelle, de la sauge, de la la"vande, du serpolet, de la sarriette, ou d'au" tres herbes agréables du même genre. On sait "en outre qu'en France et en Suisse, les fleurs "du sarrasin, de la verge d'or, de la serpen" taire qui naissent spontanément, même dans " nos jardins, plaisent assez aux abeilles; etce"pendant j’ai appris par l'expérience, que les "nôtres n'aiment pas trop ces plantes. A peine "les herbes aromatiques, comme le thim, la "lavande, la sauge, sont-elles fréquentées par "de certaines petites abeilles clont parle Vallis" niery. On pourroit dire que cela provient de " ce qu'on ne trouve pas chez nous (en Italie), "de cette espèce d'abeilles appelées Pelites 
sur les abeilles. Liv. I. Chap. II. 309 "Hollandoises, les plus petites et les meilleures "de toutes; et que les nôtres ont par consé"quent un goût plus grossier, et qu'elles ne «cherchent pas une nourriture aussi agréable. * Mais ce n'est pas ici ni le lieu, ni le temps » d'entrer dans de semblabes recherches. Ce "qu'ily a de certain, c'est que nos abeilles ne " s'arrêtent pas sur les fleurs de ces herbes, tant "recommandées par les anciens, et que les « modernes copient d'eux; quiconque y fera at«tention, en conviendra avec moi.»

Nous pouvons certifier à M. Contardi, quaprès avoir porté toute notre attention sur les abeilles pendant plus de 14 ans, nous avons lieu d'être surpris qu’il assure qu'elles daignent à peine s'arrêter sur le thim et sur la sauge. Nous ne craindrons point de dire que dans l'île de Syra, dans tout l'Archipe!, dans les îles de Candie, de Négrepont, et en général dans toute la Grèce, ces deux plantes aromatiques forment la base de leur nourriture.

On pourroit aussi dire qu'en Italie le thim, la sauge et autres plantes dont parle Contardi, ne produisent pas autant de miel que dans nos îles, ou du moins en donnent une très-petite quantité ; sans doute ces plantes ont cela de com- 
mun avcc les arbres qui, transportés dans différens pays, ne cessent pas de porter des fruits, ou en portent beaucoup moins que dans leur climat, ou dans leur terrain natal: en effet, chez nous la sauge produit une espéce de fruit excellent, que nous appelons en grec fascomilon, qui veut dire pomme rle sauge: ce fruit, dépouillé de sonécorce un peu âpre, outre qu'il est bon à manger dans sa fraîcheur, sert cncore avec le sucre ou le miel, à faire une confiture très-estimée. Quand le fruit est passé, l'ćcorce devicnt ligneuse et spongieuse; ni en France, ni ailleurs où j'ai vu de la sauge, cette plante ne produit des fruits semblables ( 1 ). On peut donc croire que

( I) Voici comment M. de Tournefort nous décrit l'origine de ce même fruit de la sange. "Les jets, dit-il, " de cette plante piqués par des insectes, s'élevent en "tumeurs dures, charnues, de huit à neuf lignes de "diamètre, presque sphériques, gris cendré, coton" nenses, d'un goût agréable, garnies assez souvent de "quelques feuilles, en manière de fraise. Leur chair est " dure et transparente, quelquefois comme de la gelée. "Les tumeurs se forment par le suc nourricier extra"vasé à l'occasion des vaisseaux déchirés par la pi" qûre."

Quoi qu'en dise M. de Tournefort, je suis persuadé 
Sur les abeilles. Liv. I. Chap. II. 3i i cette plante, ainsi que bien d'autres en Italie, ne produit pas la même quantité de miel et de cire, que chez nous. Il est même possible quill y ait en Italie des espèces de lleurs qui produisent plus de miel que le thym, la sange, et toutes celles dont parle M. Contardi; ce qui peut déterminer les abeilles à négliger et à mépriser ces plantes, pour courir après celles qu'elles leur préferent.

Ajoutons encore ici une remarque que nous avons faite sur la différence du thym de la Grèce et de celui de la France: ces deux plantesnes'y ressemblent que par leurodeur. La feuille, la fiem et le bois de l'une et de l'autre different sensiblement. Notre thym, quoique le climat chez nous soit plus chaud, ne commence à fleurir que vers le milieu de juin, ct s'y conserye, sur-

que ces pommes de sange sont les fruits natrels de cette plante, et que les piquires des inseates n'entrent pour rien dans leur formation. I'organisation uni forme de ces fruits, tant intérieure qu'extérieure, et la fréquence de ce phénomène, s'accordent mal, à ce que je pense, avec l'explication de Tournefort; d'autante plus qu'il y a à Syra quantité de ces mêmes plantes, qui constammerrt ne donnent point de fruit, quoiqu'elles soient eniieren ment semblables à celles qui en portent 
tout dans les parties les plus fraîches de l'île, jusqu'au mois de septembre, et mêne d'octobre; au lieu qu'aux environs de Paris, on le voit en fleur dès le commencement de nai, pour finir avant la fin de juin.

On doit en conclure que toutes les fleurs en général, dequelque arbre ou plante qu'elles proviennent, peuvent servir de nourriture aux abeilles, mais non pas toutes également. Ily en a qui produisent plus de miel que de cire; d'autres, plus de circ que de miel; enfin quelques - unes ne donnent ni l'un ni l'antre, mais fournissent mne autre espèce de nourriture qu'on appelle en italien ilpane delle api, en françois la matièrc

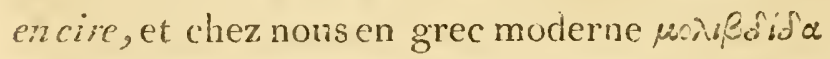

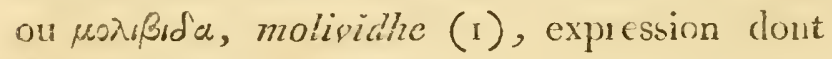

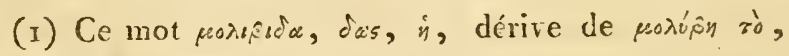
qui signifie le plomb. Les anciens, au lieu de le nom-

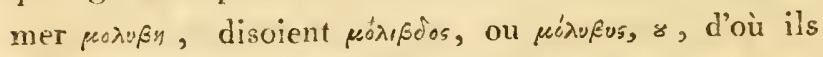
déduisoient le poovvsodıs, isos, $\dot{y}$, qui veut dire un globe, une boule, ou lame de plomb; d'où nous avons forme notre poduki ida, parce que la matiere que les abeilles portent dans leurs pattes, et que nous appelons ainsi, est de forme presque ovale, et de couleur souvent grise ou de plomb. 
Sur les abeilles. Liv. I. Chap. II. 313 nous nous servirons dans tout ce traité, toutes les fois qu'il nous arrivera d'en parler (I).

Cette différence nous est en général peu connue, mais elle l'est beaucoup par les abeilles; aussi a-t-on observé que de deux plantes éçalement flcuries, elles cuurent toutes à une seule ct abandonnent l'autre.

C'est ici le moment de rapporter une observation que nous avons faite également à Syra et en France. Nous y avons suivi plusieurs fois les abeilles pendant qu'elles voltigeoient sur les fleurs,

(1) Par la raison que les François ayant pris (moyennant la langue latine) du grec les mots cire, miel, ct propolis, c'est-à-dire, des mots grecs, x'́gos, $\mu^{\prime} \dot{\prime} \lambda \epsilon$,

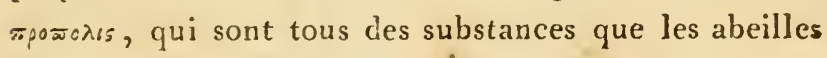
apportent dans leurs ruches. ll est juste qu'ils prennent aussi le mot de molividhe, qui est aussi une substauce dont les abeilles se nourrissent et dont elles remplissent leurs magasins, ainsi que nous le dirons en tems et lieu; d'autant plus que la supposition sur laquelle se fonde le mot, ( on l'appelle cire brute, ou matière en eire, croyant que de cette matière digérée, les abeilles forment la cire, dont les Auteurs françois se servent pour exprimer cette matière que nous appelons molividhe), est fausse, ainsi que nous le ferons voir, lorsque nous parlerons de l'origine de la cire. 
nous avons remarquéqu'ellcs suivoient constamment une même espèce, l'une après l'autre, jusqu’à ce qu'elles fussent chargées ou de mielon de molividhe : elles ne changeoient jamais de fleurs, quoiqu'il s'en trouvât anx environs de plusieurs autres espèces, qui auroient pu fournir également ì leur nourriture. D'oùl'on peut conclure que les abeilles ne se chargent, dans une course, que des poussières ou du miel d'une sorte de plante.

A l'égard des petites Hollandoises, on ne les connoît pas à Syra: par-tout où nous avons vu des abeilles, nous les avons trouvées semblables aux nôtres; mais lorsque nous parlerons des abeilles communes, nous dirons la raison bien simple, pour laquelle on voit dans les vieilles ruches quelques abeilles plus petites que les autres.

P.S. Avant de finir ce chapitre, nous ajortterons quelques observations, tirées de l'ouvrage de M. Duchet sur les abeilles : clle entrent naturellement dans le sujet que nous traitons.

Cet Auteur, vers la fin da Chapitre IX, demande si on peut augmenter le produit des abeilles en miel et en cire, et il répond affirmativement: il ne faut pour cela que s'appliquer à la multiplication des fleurs, des arbres et des plantes. Plus nous aurons de ces espèces, 
sur les abeilles. Liv. I. Chap. II. 3i 5 et sur-tout de celles qui fournissent davantage, plus nos ouvrières trouveront de matériaux propres à bâtir leurs rayons, et à les remplir de miel.

"On plante, on cultive les mûriers, uniquement pour les vers à soje: on en cueille journellement les feuilles pour les distribuer à ces. insectes, arec une assicluité fatigante. Les abeilles au contraire nous laissant toute l'utilité de leur travail, ront elles-mèmes recueillir ce qui leur est nécessaire : nultiplions nos arbres fruitiers; plantons des saules qui dessechent les fonds, et fleurissent les premiers; élevons des tilleuls qui fleurissent les derniers, et qui donnent beaucoup; et si nous faisons bien, nous cultiverons en plus grande quantité des fêres, des haricots, du bled-sarrasin, dans différentes saisons. L'auteur du recucil de pièces économiques prétend qu'un arpent de terre semé en féres ou en haricots, et cultivé selon la méthode qu'il prescrit, pourroit rapporter 998 livres de France; quand on en déduiroit 200 livres pour les frais de culture, qui, selon lui, n'iroient passiloin, n'y trouveroit-on pas encore un grand avantage, puisque les abeilles profiteroient du miel? Pourquoi ne s'appliqueroit-on pas à la cul- 
ture des plantes oléagineuses, des luzernes, des serpolettes, sur-tout de celles dont les fieurs se succèdent pendant long-temps, et qui, à leur utilité commmne, ajoutent celle d'être néces. saires à nos insectes? Voilà une vaste carrière ouverte aux amatenr's. 》

"Les espérances, dit le même Auteur dans le chapitre suivant, qu'on auroit conçues du produit des vers à soie, élevés dans ce payz-ci (en Suisse), avec les mûriers qui s'y trouroient, il y a dix ans, auroient paru bien minces à des yeux un peu éclairés. (Qu'a-t-on fait? on s'est conduit plus prudemment : pour faire prospérer cette branche d'économie, on plante et on at planté une grande quantité de mûriers, qui sont la base de cette éducation; ainsi, en procurant la multiplication de la nourriture, on assure celle des vers et de la soie; conduite louble et pleine de sagesse. De même la multiplication des arbres et des plantes, dont les fleurs servent à la pâture des abeilles, entrâ̂neroit celle des essaim;, de la cire et du miel, arec la différence que les mûriers ne servent presque qu'aux insectes qui filent la soie, le bois n'en étant même pas bon à brûler; an lieu que les sources nouvelles din miel que nous 
Sur les abeilles. Liv. I. Chap. II. 3 r 7 multiplierions, nous laisseroient en entier tous les avantages qui en découlent, comme ceux du bois, des fruits, des huiles, des légumes, des foins, etc. On est encore borné pour les vers à soie à deux on trois espèces de mûriers, an lieu que pour le miel on peut compter des milliers d'arbres, de plantes on arbustes. (Qnelle infinité de soins ne faut-il pas tous les jours pour élever les vers à soie, pendant que les abeilles, vont elles-mêmes, et quelquefois jusquà phusieurs lieues de leur demeure, se procurer leur subsistance?"

"Le profit des vers à soie seroit très-modique sans les nouvelles plantations, et sans elles, celui des abeilles sera toujours très-considérable ; mais si nous pouvons l'augmenter facilement, par la multiplication des sources de la cire et du miel, ne négligeons pas un tel moyen, qui, loin d'être nuisible à l'agriculture, en augmenteroit encore le produit dans ses autres branches, tandis que les plantations de mûrier's ne leur sont pas toujours favorables. Un fort tilleul, qui n'occuperoit qu'un espace de quelques pieds, qui pourroit même venir parmi des rocher's où il y auroit un peu de terre, fourniroit plus à nos insectes pendant un mois, que plusicurs 
arpens en champs ou en mauvais marais. Si on en plantoit une centaine dans un village, à la place de tant d'autres qui ne sont bons à rien, que de miel ne fourniroient-ils pas, et pendant combien de temps! Que de bons arbres pourroient être élevés dans de vastes communes presque stériles! Si on desséchoit les marais par des fossés et des chaussées, les mauvais plants s'y changeroient en plantes salubres, le bétail s'en porteroit mieux, et le miel seroit plus abnndant, et d'une meilleure qualité. Si, parmi les rochers, on plantoit des chênes, des érables, des fênes, des tilleuls, etc. quels avantağes n'en retireroit-on pas? par l'établissement des prairies artificielles, on auroit plus de bestiaux et plus de laitage. Les bons terrains pourroient être couverts d'arbres fruitiers de toute cipèce, et sur-tout de pruniers, comme les plus lavorables aux abeilles. Cet arbre est fertile, et son fruit excellent. On pourroit encore se procurer d'autres arbres qui nous manquent, et qui sont préféréspar nos insectes, comme l'érable du Canarla, le chêne verd, l'ar bousier, etc. Ne pourroiton pas fournir aussi in plus grand nombre de haics vives, dans lesquelles on feroit dominer les plants les plus agréables aux abeilles? Que de plantes 
SUR les abeilles. Liv. I. Cap. II. 319 utiles ne pourroit-on pas cultiver? Je ne fais qu'effleurer une matière qui présente le plus vaste champ aux observations des personnes zélées pour le bienpublic. «Le rai: onnement de M. Duchet est juste ct très-exact: on n'a besoin que d'entrer dans quelques jardins particuliers, pour s'en convaincre. J'ai observé, sur-tout dans celni de M. Le Monnier, premier médecin du Roi, une infinité d'arbres et de belles plantes étrangères, qui, en formant une superbe décoration, viennent parfaitement clans ces climats, et sans beeucoup de soin. Ils pourroient être d'un grand avantage pour nourrir nos insectes; ce qui devroit exciter le zèle des bons citoyens, pour les multiplier. On en trouveroit la plus grande facilité avec M. Le Monnier , qui, rempli du plus grand zèle pour le bien public, se feroit un plaisir de fournir les graines de tout ce qu’il possède, pour en multiplier les espèces dans tout le royaume. 


\section{H A P I T R E II I.}

Liste des principaux arbres, arbustes et fleurs, qui serven à la nourriture des abeilles.

L'amander. Ses fleurs viennent de bonne heure, et il semble qu'elles aient une odeur de miel. Les abeilles y font une copieuse récolte.

Le cerisier. C'est un des arbres qui plaisent le plus aux abeilles; ses fleurs donnent bearicoup de miel, et une senle en contient dssez pour remplir la vessie d'une abeille ( 1 ).

(1) Cette abondance de miel que M. Contardi attribue au cerisier, me semble extraordinaire; au reste, n'ayant fait aucune observation particuliere sur cet arbre, je laisse aux gens instruits, ct qui ont de l'expérience à en juger, en remarquant d'aillcurs que quoique le cerisier n'ait pas cette fécondité en miel dans tous les pays, il pourroit l'avoir dans le pays de l'auteur.

L'ABricotier. 
sur les abeilles. Liy. I. Chap. III. 32 I L'abricotikr. Sa fleur est fréquentée par les abeilles.

Le Noisctier. Ses fleurs mâles ou chatons contiennent beaucoup de miel. Elles sont les premières de toutes les fleurs, qui, par un tems doux, quoique la terre soit encore couverte de neige, fouraissent dés les premiersjours de fév rier de la nourriture aux abeilles. Cependant la fleur de ces arbres doit donner peu de miel, puisqu'elles ne la recherchent que faute d'autre aliment.

LE POMmier. Il donne une fleur sur laquelle les abeilles sarrêtent volontiers. La manière de cultiver cet arbre est assez connue; ainsi je n'en parlerai pas.

LE PEUPLier. Il est extrêmement recherché des abeilles; il supplée au tilleul dont nous manquons. Le peuplier abonde par-tout; mais on prétend qu'il rend la cire onctueuse et résineuse. Il y en a die plusieurs espéces.

Le trembre. Lesabeilles font de bonnes provisions sur les chatons de cet arbre; c'est de là peut-être qu'elles tirent à Syra leur propolis. Cette résine ou gomme est tenace et de couleur brune. Les abeilles en bouchent les fentes de leur ruche. Le tremble se plaît dans une terre fine et grasse, dans: les. lieux humi-

Tome $I$. 
des et frais; c'est pourquoi on le plante dans les vallées profondes, et le long des rivières et des torrens. On peut multiplier cet arbre de graine, ou de rejetons; cependant la maniere la plus courte et la meilleure est de planter ses branches dans des fosses de trois pieds de profodeur, creusées avec un hoyau, ou faites simplement avec un pieu ou un bâton, qu'on enfonce dans la terre. Mais au lieu de cette méthode, il seroit plus utile de faireces fosses comme pour les autres petits plants, et quand le trou est fait, on y met la branche, et on remplit de bonne terre le vide qui est autour, en observant que cette terre la touche de tous les côtés, et on l'arrose souvent: par ce moyen, le plant pousse facilement des racines.

LESAULE. Les abeilles font sur les chatons de ses fleurs une abondante récolte. Cet arbre est d'une grosseur médiocre, d'un bois léger, flexible et difficile à rompre. Il se plaít dans l'eau, et devient plus gros dans les endroits humides; malgré cela il n'est pas ennemi des lieux secs, sur-tout des terres fortes et grasses. Il ne croît pas bien, s'il a les racines dans l'eau; mais il réussit dans une terre humide, comme dans les fossés, les digues, les prés et dans les endroits qui sont 
SUR LES ABEILles. Lrv. I. Chap. III. 323 ₹empérés et frais. La manière la plus avantageuse de multiplier le saule, est de pianter les branches, comme nous l'avons dit pour le peuplier.

Le sureau. Nous n'avons jamais vu d'abeilles sur ses fleurs; cependant quelques persomes disent qu'elles en retirent beaucoupde miel, et peu de cire. Il y en a une espèce qui fleurit en juir. Le sureau est un arbuste d'une grandeur mécliocre. On en voit de deux espèces, I'une qui donne desfleurs très-blanches, tellement disposées en grappes, qu'elles forment un globe. II s'appelle en italien sambuco-roseo, en françois, sureau-rose. L'autre produit une grande quantité de fleurs blanches qui ont un peu d'odeur et disposées en ombelles, larges d'environ une palme : elles produisent des bayes noires, remplies d'ui suc reuge foncé. Ces deux espèces de sureau se multiplient en plantant des branches avec ou sans racines.

LA Bruytere. Elle fleurit dans les mois de juin et juillet, et donne beaucoup de miel aux abeilles.

LE BUIs, l'arbousier, le cornouiller, l'orme, le pêcher, le tilleul, lolivier, le chamerodhendron sont tous décrédités par les an- 
ciens, qui ont voulu donner it entendre que leurs fleurs rendoient malades les abeilles. Chacun croit ce qu'il lui plait. Pour moi, je suis persuadé, au contraire, que les fleurs du tilleul particulièrement plaisent beaucoup aux abeilles, et qu'elles y recueillent beaucoup de miel. M. Duchet a observé que dans les endroits où cet arbre abonde, les abeilles se conservent trèsbien, et ont toujours une bonne provision de miel. Le tilleul est un grand arbre assez commun; il se renverse facilement et ne dure pas. Il vient bien dans une terre grasse et un peu humide. Les meilleurs sont cenx qu'on a multipliés de graines. Lorsqu'on veut faire reprendre desplantes sans racines, il faut choisirles plus jeunes, et les laisser pendant quelque temps dans la mêrme position. Au surplus, pour ce qui est des plantes dont les fleurs sont nuisiblesau miel, voy.lech. 5 ci-après.

L'A b b Usier. C'est un arbuste qui s'élève peu, qui conserve ses fenilles, et qui produit nu fruit un per ovale, d'un jame tirant sur le rorgge, de la grosseur d'une cerise. Les Paysans l'appellent en italien l'albatro.

Le cormier est un petit arbre; mais qui jette beaucoup de branches : il croît facilement dans 
Sur les abtilles. Lit. I. Chap. III. 32 i. toutes sortes de terrains; on le prend dans les. bois, parce qu'alors il vient plus vîte.

L'ORMe est un arbre de haute futaye et droit; la meilleure méthode de le multiplier, est de mettre en février les branches clans la pépinière, qui doit être d'une terre forte et bien fumce : decette maniére, elles poussent des racines latérales, et l'écorce devient lisse et unie, si on laboure la terre deux fois par an.

M. Contardi dit encore, en parlant des fleurs, que les polipétales on les monopétales en gaénéral plaisent aux abeilles. On y distingue facilement la liqueur miellée: il suffit de les arracher de leur calice et de leur sucre; alors on sent la douceur du miel. Mais les plus agréables aux abeilles, sont les campaniformes, les cruci. feres, et les ombelliferes. De la classe desfícurs en cloches, sont la bruyère, le liseron, la mauve, la citronelle, le houblon, et quelques autres semblables. Elles fournissent une grande nourriture aux abeilles, peut - être parce que la licueur douce, contenue dans ces fleurs, est mieux renfermće, et qu'elle s'évapore moins. Le chou, la roquncte, la rave, et d'autres sembla. bles sont de la classe des cruciferes. Le persil, le panais, le fenouil, eont de la clarso des oma 
belliferes : les plus petites de ces fleurs contiennent, sclon le sentiment d'Odierna, une grande quantité de micl. Nous nommerons encore ici quelques autres herbes et arbustés, dont les fleurs sont les plus fréquentées par les abeilles.

L'Althéa dome des fleurs en mai et les porte pendant trois mois. Les Botanistes l'appellent rilheafrutex, pour la miecix distinguer des autres.

LA BÉTOINE. Sa tige est haute d’un pied et plus: elle est d'un goût aigre et amer, et Gallo la regarde comme la plus parfaite de toutes les herbes.

LA BOURRACHE, herbe de jardin très-connue, dont les feuilles sont vertes, larges, longues et couvertes d'un poil très-rude. Elle produit beancoup de fleurs, et les conserve pendant plusicurs mois. Ses fleurs contiennent beaucoup de miel, et les abeilles les fréquentent roJontiers. (1).

Lechou, comme aussi le choufleur, le bro-

(I) J'ai observé l'année derriere dans un jardin à Versailles, que celte plante grandissoit et s'étendoit beaucoup; et que, pendant plusieurs mois, les abeilles qui viennent s'y nourrir, y forment une espece d'essaim continuel, du matin au soir. 
Sur les a Beilles. Liv. I. Chap. III. 327 colis, et les fleurs de toutes ces espèces plaisent beaucoupaux abeilles.

CÉRINThéc. C'est le mélinet des François, donne beaucoup de cire aux abeilles.

Écнyum, la vipérine. Herbe très-agréable sux abeilles.

Le lierre. Elles en tirent beaucoup de miel. L A DENT DE LION, les fèves, pois et autres plantes de cette espèce; produisent des tleurs que les abeilles aiment beaucoup.

Tournesol ou hélyotrope.J'ai souvent observé cette fleur; je n'y ai jamais vu aucune abeille, mais bien une quantité de mouches d'une autre espèce ; cependant je ne doute pas que les abeilles ne pussent trouver de la nourriture sur ses fleurs; car elles ont quelque chose de résineux, qui donne une odeur agréable, et qui doit leur fournir beaucoup de propolis.

LE FRAmborsier. Les abeilles sont tres-avides de sa fleur. C'est une plante épineuse qui se multiplie de rejetons qui viennent, et se transplantent au printemps à deux pieds de distance les uns des autres.

LE LIN. Au printemps, lorsqu'il est en fleur, les abeilles $y$ abondent, et en tirent beaucoup de miel. 
LA MAUre, et la rose d'outremer (malvarosca). Sesíleurssont tiès-utiles aux abeilles; il ne faut pas qu'clles soient dans le roisinage des ruches, parcequ'elles attirent ces petits papillons qui produisent les vers dans les rayons. Dans l'Archipel, on fait beaucoup de cas de la manrve, et l'on croit qu'elie produit beaucoup de cire.

La luzerne. C'est une espéce de treffle dont - ya fleur est très-agréable aux abeilles : elle se sème à la mi-mars très-profondément dans nne terre plutôt légiere que forte, et exposéc an midi. Dans les lieux très - hunides, on sème trois quarts de resce avec un quart de luzerne. Ce mélange la garantit de l'ardeur du soleil qui Ini fait beaucomp de tort.

Cresson. Celui qu'on appelle cresson de fontaine, He:urit en juillet et en août; il clonne une bonne nuirriture aux abeilles.

L'origan. Plante sauvage qui croît dans les lieux montueux, d'où il vaut mieux la trans. planter que de la semer. Ses feuilles sont un peu velues, et plus grandes que celles de la marjolaine. Ses fleurs, qui viennent en touffes, sont rouges et quelquefois blanches : les abeilles en Eirent une bonne nourriture.

LE I I O T simple, comme aussi une espece 
SUR les abeillis. Liv. I. Chap. III. 329 de renoncule simple et sauvage, qui croît chez nous sur les montagnes et par-tout, produit beaucoup de molividhe pour les abeilles; la renoncule au commencement du printemps, et le pavot vers la fin.

LEPANAIS est une plante dont la racine est d'un gôtt âcre. Il y en a de plusieurs espèces: elle fleurit en juillet et août.

Pouliot. Plante très-basse et dont les fleurs sont rouges et rondes. Elle croît, dans la campagne, dans les lieux incultes et aquatiques. Elle fleurit en été.

- La RAve, ainsi que toutes les plantes quilui ressemblent par les feuilles et les fleurs sont très-bonnes pour les abeilles.

LA ROQUETTE est une herbe d'un goût âcre. On la sème dans le printemps; elle ne craint pas le froid. La saurage a les feuilles plus étroites, plus découpées et d'un goût plus piquant.

Le romarin. On le multiplie, en coupant, vers la fin de márs ou au commencement d'avril, les jeuncs branches avec un peu de racine, s'il est possible, et en les transplantant dans une terre grasse, peu humide, et exposée à la chaleur; elle périt fácilcment au froid. On plante le romarin dans les jardins, près des ruches, parce 
que les abeilles $e n$ aiment beaucoup la fleur. Elle les entretient saines, à ce que l'on croit, et donne une bonne odeur au miel. Elle fleurit deux fois l'an dans le printemps et dans l'automne.

LE SÉNEVÉ. Ses fleurs durent long-temps: le plus recherchédes abeilles, est le sauvage, qu'on appelle en italien enucaggine, et qui croît en grande abondance dans les champs. On le cultive dans les jardins; on le sème fort clair. Dans le printemps, il lui faut de la poussière au pied, et l'été de l'eau.

Le Trèrle. Celui qui a la fleur rcuge, contient du miel. Cela est connu des enfans, qui le cueillent pour. le sucer.Ils sucent aussi les fleurs de chèvre-feuille. Le trèfle à fleurs blanches convient le mieux aux abeilles, parce qu'ayant le calice plus court, leur trompe s'y ajuste mieux. Dans le Levant, la fleur du treffle est jaune, ct après elles le thim et la sauge; e'est une des meilleures nourritures, lorsqu'il vient bien, et enabondance.

L'AUbÉpine fleurit de bonne heure. Ses fleurs plaisent beaucoup aux abeilles. C'est une plante épineuse qui produit des fruits semblables au raisin. On la multiplie par des branches détachées qui ont un peu de racine. Il faut remuer la terre tous les ans autour du pied, et n'y laisser 
SUR les abeilles. Liv. I. Chap. III. 33i croître aucune herbe qui puisse lui ôter la nourriture, et la dépouiller de toutes les branches vieilles et sèches.

La vesce donne une fleur qui produit beaucoup de miel. Souvent on voit les abeilles sur les cosses de la vesce, sans toucher aux fleurs. On croit qu'elles y recueillent une grande quantité de miel. (Dans le chapitre qui traite du micl, on parlera de celui que les abeilles recueillent sur les feuilles ).

LE BOUILLON BLANC produit en juin et juillet beaucoup de fleurs qui contiennent une grande quantité de miel. Les enfans les cueillent pour les sucer.

Les fleurs de la citronnelle, du melon et d'autres semblables fournissent une abondante récolte aux abeilles,particuliérement en molividhe.

Avant de finir ce chapitre, nous rapporterons une pensée très-juste de Contardi sur les herbes qui viennent dans les champs, et qui sont contraires au goût des abeilles.Si on vouloit clétruire,ditil, de pareilles herbes, on auroit tort de suivre en cela le sentiment des anciens qui proposoient de le faire; puisque les abcilles (comme tous les autres animaux ), ne courent pas aux herbes qui leur sont pernicieuses, ou qui leur déplaisent. 


\section{I A P I T R E I V.}

Calalogue des aulres plantes et arbrisseanx étrangers, dont les fleurs sont utiles aux abcilles.

C'es tans les jardins de M. Le Monnier, premier mélecin du Roi, au grand Montreuil près de virsailles, que j'ai découvert un grand nombre d'alubres, et de plantes ćtrangrèeses, dont les fleurs étoient fort recherchées des abeilles. Ce savant médecin, dont toutes les vues sont uniquenent dirigées vers ce qui pent servir à l'humanité et à liutilité publique, se feroit un plaisir d'olfrir à tous les amateurs et aux curicux, des graines de tuntes les plantes qu'il cultive, et dont voici la liste.

L'ancholie, l'aiglantine, la colombine, la gar Jantine, ou gyants de N. D., est une plante dont la tigge s'élève a la hauteur de deux ou trois pieds; les botanistes l'appellent aquilegia floro simplice, aquilegia necuriis incurvis. Cette plante, qui croît aaturellement en France dans 
SUR les abeilles. Liv. I. Chap. IV. 333

les bois, fleurit an mois de juin et de juillet: elle se multiplie facilement de graines; on en voit, dans les parterres à fleurs bleues, violettes, rouges, couleur de chair, blanches etc.

Andromada. Il y en a plusieurs espèces; les plus fréquentées par les abeilles sont: $I^{\circ}$. la racemosa ou à grappes, qui croît communément dans les jardins, à la hauteur d'environ quatre pieds; ses fleur's sont petites, blanches, globuleuses, piIulliformes, tournées d'un même côté, et vers la terre, ce qui empêche leur miel d'être altéré par la pluie. Cet arbrisseau fleurit en mai et en juin.

$2^{\circ}$. La masiana, qui est une des plus belles dans ce genre, et peut-être celle qui porte les fleurs les plus grandes, s'élève ordinairement à la hauteur de deux pieds. Ses fleurs sont blanchâtres, grandes, pédonculées; elles se forment de quatre ou huit ensemble par petits bouquets, et paroissent dans les mois de mai et de juin. Cette plante croît naturellement daus le Maryland et dans la Virginie. Elle se multiplie de graines.

$3^{\circ}$. LA PANicUla lata; c'est un arbrisseau qui s'élève à la hauteur de quatre ou cinq pieds. Ses fleurs sont d'un blanc un peu pâle, ct nais- 
sent sur de petits rameaux nuds et disposés en épis; ses fleurs paroissent dans les inois de juin et juillet. Toutes ces espèces se multiplient de graines.

Agripaume; cette plante bien cultivée s'élève quelquefois jusqu'à quatre pieds. Ses fleurs sont petites, blanchâtres, ou légèrement purpurines. On la trouve dans les haies, et elle fleurit dans le mois d'août.

Arocin-gobe-mouches; cette plante, quand elle est en fleur, est très-jolie; sa tige d'environ un piecl et demi, est herbacée et rougeâtre; ses fleurs naissent par bouquets presqu'ombeliformes. Elle croît naturellement daus le Canada et dans la Virginie; clle fleurit dans les mois de juin et juillet, et se multiplie de graines, et de racines : ses fleurs sont asscz fréquentées par nos insectes. On lui a donné le nom de gobe-mouches, parce que les monches avides du suc miclleux qui se trouve dans le calice de ses fleurs, insinuent leur trompe par le passage étroit qui se trouve entre les corpuscules qui entourent les ovaires, et lorsqu'elles venlent la retirer, elle s'y engage davantage; l'insectc alors s'y trouve pris comme dans un piège, et y périt. Il n'en est pas de même de l'abcille; sa trompe 
SUR Les ABeilles. Liv. I. Chay. IV. 335 étant plus lisse ne s'élargit pas à son extrémité comme celle des mouches communes, et elle retire le miel de cette fleur sans aucun rlanger. Apocis à feuilles d'androsemum; c'est une plante dont la racine est vivace : ses graines mûrissent rarement dans ce climat; mais elle se propage par les jets des racines. Cette plante fleurit en été; elle aime une terre légère et seche; il faut peu l'arroser dans la belle saison et jamais dans l'hiver.

Asclípiade de Syrie; elle est à fleurs blanches en dedans, et d'un rouge clair ou rose en dehors:les jardiniers la nomment apocin du Canada. Cette plante fleurit deux mois de l'été ; elle réussit fort bien en pleine terre, et à des expositions un peu ombragées, et se multiplie de semences. Asclépinde de Syrie ou de Canada à larges feuilles. Cette plante produit une espèce de ouette, et doit être exclue des jardins, dit un auteur, parce qu'elle trace tellement, qu'elle est fort difficile à détruire. Cependant ses fleurs sont très-utiles et très-agréables aux abeilles. Agripaume. Cette plante haute de deux à trois pieds, s'élève même jusqu'à quatre, lorsqu'on la cultive : ses fleurs sont petites, blanchâtres, ou légèrement pnrpurines. On la trouve 
dans les haies, et elle fleurit dans les mois de juillet et d'août.

Calma angrustifolia, ct latifolia. Cet arbuste vient quelquefois à la hauteur de quatre à cinq pieds. Ses fleurs sont au bout de ses branches en bouquets arrondis; il fleurit en juin, se multiplie desemences, de marcotes, on de boutures, et nous vient du Canada. Il aime un terrain sabloneux, humide, et ombragé : quand il est jcune, il faut le garantir des grosses pluies.

Campanule. J'en ai vu plusieurs espèces dans les jardins de M. Le Monnier, et toutes très-fréquentées par les abeilles. Cette plante se multiplie degraines légérement enterrées, et que l'on seme en automne ou au printemps. Toutes les campanules aiment none terre bien meuble.

Clematite, ou herbe aux guenx; c'est un arbrisseau grimpant, qui pousse beaucoup de sarmens très - flexibles. Ses fleurs en bouquets axillaires et terminaux, composées ordinairement de trois bonquets partiels, sont petites et blanchics : elles ne paroissent qu'au mois de juin. Cet arbrisseau a huit ou neufs variétés, et se multiplie ordinairement de drageons; excepté celui qui est à fleurs doables. Ses manottes prennent racine dificilement : tont terrain leur convient. 
SUR les abeilles. Liv. I. Chap. IV. 337

Catalia. Ses fleurs par gros bouquets sont odorantes; ce bel arbre, qui fleurit vers la fin de juillet, se multiplie de marcottes, de bou. tures, et de semence : il aime l'ombre.

Cornouiller. Il y a plusieurs espèces de cet arbrisseau, toutes très-fréquentées par les abeilles, et sur-tout la blanche de Virginie. Il se perpétue par les semences, les greffes et les marcottes : toute terre lui convient, et il lui faut peu de soleil.

Clethra. Cet arbrisseau se plaît singulière. mentdans les terres aquatiques; et à Parisil vient en plein air : on peut l'élever de semences, et le multiplier par des marcottes. Il produit, dans les mois de juillet et d'août, de jolis épis de fleurs blanches, qui servent de décoration aux bosquets d'été : il est utile pour la nourriture des abeilles, qui le fréquentent avec beaucoup d'avidité.

Diospyros lotus, ou Guajacana, Plaqueminier ou Piaqueminier. Lesfleurs de cet arbre sortent une à une desaisselles des feuilles, et paroissent dansle mois de juin. Il y en a trois espèces. $I^{\circ}$. Le guajacana-plaqueminier à petit fruit. $2^{\circ}$. Le guajacana angustiore folio, plaqueminier à feuilles étroites et à petit fruit. $3^{\circ}$. Le guajacana, ou

\section{Tome $I$.}


pishamin virginianum, plaqueminierde Virginie, à gros fruit. Les plaquıcminiers s'élèvent de semences; le premier donne des rejettons, quand il est un peu gros.

Digitale d'Espagne. Cette plante fleurit en mai et juin. Elle fait un très-bel effet dans les parterres par la beauté de ses fleurs. En France la commune se trouve par-tout.

Hydrangea. Cet arbrisseau vient de la Virginie. Ses fleurs en bouquet terminal, ou en parasol, paroissent vers la fin de jnin jusqu'au mois de juillet; elles sont blanches et fort petites. Il se multiplie de marcottes et de drageons: ii lui faut peu de soleil, et tous les terrains lui sont favorables.

Hyppophae rhamnoides. Il y en a de deux espèces, celle d'Europe, et de Canada. Cet arbrisseau fleurit au commencement de mars. Quoiqu'il vienne assez bien partout, il préfere un terrain sablonneux. On le multiplie par des semences, de marcottes et de boutures.

Hyppericus acrrum; Mille-pertuis. Cet arbuste fleurit dans les mois de juillet et d'août; il se multiplie aisément de semences, et de drageons en racines. Ses fleurs sout jaunes et agréables : on peut l'employer pour la técora- 
sur les abellies. Liv. I. Chap. IV. 339 tion des bosquets d'été. Il n'est difficile, ni sur le terrain, ni sur l'exposition.

MÉlrsse. Cette plante, qui fleurit dans les mois de juillet et d'août, se multiplie communément de plants enracinés, quoiqu'elle vienne également de graine. Les vieux pieds produisent beaucoup de rejetions; on les plante au mois de mars en bonne terre bien fumée, et un peu à l'ombre; ils réussissent fort bien auprès des arbres fruitiers. Cette plante dégénère quelquefois en une espèce dont l'odeur est puante. On la coupe deux fois tous les ans, en été et en automne. Elle repousse bientôt et se soutient fort bien, sans craindre le mauvais temps.

Mélinot, irifolium melilous officinalis. Cette plante est bisannuelle, et croît dans les haies et les buissons. Elle produit beaucoup de miel, et les abeilles la recherchent avec empressement, sur-tout celle à fleurs blanches; celle-ci est sifort de leur goût, qu'elles ne la quittent pas de toute la journée pendant les mois de juillet et d'août : elle leur seroit aussi avantageuse que le sarrasin, parce qu'elle doit produire un miel de meilleur goût. Cetteplante donne aussi 
une grande quantité de fourrage, ce qui doit encourager à la cultiver.

ITEA VIRGINICA. Cet arbrisseau vient naturellement en Canada et daus la Louisiane. Il fleurit dans les mois de juin et de juillet. Il se multiplie aisément de marcottes, s'accommode de toute espèce de terrain, de toutes les expositions, et n'exige aucune culture particulière.

Luxaire, ou le bulbonac bisannuel. Ses fleurs sont semblables à celles de la julienne simple. Cette plante fleurit au mois d'avril, et se multiplie elle-même de semences.

LE MUGUET, ou lis des vallées, est une plante qui sélève à un clemi pied, qui porte plusieurs fleurs disposées en grappes et rangées d'un seul côté. C'est le lilium convallium album. Elle croît dans les bois, dans les vallées, dans des licux ombragés et humides. Elle est commune dans plusieurs provinces de France.

Olivier de Bohême, olivier sauvage, Elceagmus angustifolius. C'est un grand arbrisseau, ou petit arbre, qui flcurit au mois de juin.Ses fleurs sont petites et jaunes, très nombreuses, et très odorantes. Il se multiplie de marcottes et de boutures, et vient dans tous les terrains.

Trô̂NE, ligustrum. Ce joli arbrisseau est très- 
Sur les abeilles. Liv. I. Chap. IV. 3 fi commun dans les bois. Ses pctites fleurs en grappes terminales un peu pyramidales, serrées et bien garnies, paroissent en mai et juin. Il se multiplie de semences et de marcottes.

Sorhor a japonica. C'est un grand et très-bel arbre, que l'on voit en fleurs dans le jardin de M. le Monnier dans le mois d'août. Ses feuilles sont assez semblables à celles de l'acacia. Ses fleurs en épis axillaires, sont d'un blanc un peu jaune, papilionacées; elles produisent un grand nombre de semences sphériques, par lesquelles on multiplie cet arbre. Il s'accommode de toute espèce de terrain, pourvu qu'il soit exposé au grand soleil. Cet arbre que j’ai vu con. vert d'abeilles, doit leur être très-utile, sur-tont dans une saison oì la campagne manque de fleurs.

SPIR Æa. C'est un petit arbrisseau haut de deux à trois pieds. Ses fleurs qui paroissent en juin, sont purpurines, presque blanches, par bouquets ou épis terminaux, et serrées. Ce joli arbrisseau a une variété dont les fleurs sont rouges, et s'onvre dans le même temps.

Le spiræa d'Espagne à feuille de mille-pertuis s'élève un peu plus. Ses fleurs sont blanches et plus petites que celles de l'autre; il y en a 
3 1. TR A T TE COM F E T

une variété qui se distingue par les feuilles dont l'extrémité est crenelée ou dentelée.

Le spirza à feuille d'obier, ne différe de l'obicr même, que parce que les feuilles du spiræa sont alternes. Il donne au commencement de maide jolis bouquets de fleurs.

Les spiræa se propagent par les marcottes ct les drageons; ils aiment une bonne terre un pei humide, et craignent le grand soleil.

Sumac, rhus coriaria; c'est un arbrisscaudon ${ }_{t}$ la hauteur excècle rarement plus de cinq pieds, dit un auteur; mais j'en ai vu qui en avoient plus de dix. Ses fleurs rassemblées en grand nombre et serrées en épis terminaux sont blan. châtres, fort petites, et composéescommecelles du fastet.

Il y a un sumac de Virginie dont les fleurs et le duvet des baies sont rougcâtres.

Le sumac de Canada a les feuilles lísses de deux côtés, et blanches en dehors; ses fleurs sont de conleur presquie herbacée. Touteś ces sortes de sumac sont fréquentées par lés insectes, et se multiplient par les rejettons. Elles viennent bien dans tous les terrains, et demandent beancoup de soleil.

Filipendule. Cette plante vivace fleurit dan $_{S}$ 
SUR les abeilles. Liv. I. Chap. IV. $37^{3}$ les mois de juin et juillet. Elle se tronve dans plusieurs provinces de France, et se multiplie par les pieds ćclatés en automne, et au printemps.

Thaletron, ou thalictron commun; c'est la rhue des prés. Ses petites fleurs naissent aux sommités des tiges et des rameaux. Cette plante croît dans les prés, dans les lieux humides ou marécageux, ou le long des ruisseaux. Elle fleurit en été.

Prinos verticillé; c'est un arbrisseau de Virginie, qui s'élève à huit ou dix pieds. Ses fleurs sont axillaires, quelquefois solitaires, quelquefois de trois ou quatre en bouquet. Cet arbrisseau a beaucoup de rapport avec le houx; il est rave, et se muliplie très-difficilement par les semences, dit assez mal à propos un auteur, car il réussit aisément : il fleurit en juin et juillet.

LA REINE DES P'RÉS, spirca ulmaria; c'est une plante vivace. Ses fleurs sont petites et blanches; elle vient dans presque toute la France, dans les prairies, sur le bord des ruisseaux et autres lieux humides: elle fleurit en juin.

Vionne, coudre moinsine ou mansienne. $V i$. burnum Lanıana. C'cst un arbrisseau fort commun dans les bois. Ses fleurs sont blanches et 
rassemblées en grand nombre, en grosses ombelles terminales; il y en a plusieursespèces, et toutes fort agréables pour la décoration des jardins; les viornes flemrissent en juin, se multiplient de semences, de marcottes et de greffes. Elles s'accommodent de toute espèce de terrain. Caprier ordinaire. Cette espèce est la plus commune, et la seule qui croisse naturellement en Europe; elle intéresse non-seulement par sa beauté, mais encore par l'nsage que l'on fait des boutons de ses fleurs, et par le pâturagace qu'elle fournit aux abeilles. Cette plante crôtt dans les parties méridionales de l'Europe, particulièrement en Italie, en Provence et en Languedoc, dans les murailles, les lieux pierreux et les fentes des rochers. Les abeilles aiment beaucoup les fleurs de cette plante.

SCLARÉE, orvale, ou toute-bonne des prés; c'est une plante vivace. Elle pousse une ou plusieurs tiges; leurs sommités portent des fleurs verticillées, disposées comme en épis longrs. Cette plante croît le long des chemins, sur le bord des champs, sur les collines herbeuses, et dans les prés hauts ou bas. Elle fleurit aux environs de Paris dans les mois de juin et juillet.

Statice de Sibérie. Plante charmante, qui ou- 
sur les abeilles. Liv. I. Chap. IV. $3 q^{5}$ tre sa beauté particulière, produit une quantité de nourriture pour les abeilles; aussi la fréquentent-elles singulièrement. Elle se propage de graines, et fleurit dans le mois de juin.

VITex, agnzus casizıs. C'est un joli arbrisseau qui réussit assez bien dans tontes sortes de terrains; il se conserve en pleine terre dans les environs de Paris, et se multiplie très aisément de semences; il y en a une espèce à petites fouilles, qui fleurit vers la fin de juillet, et qui se conserve en fleurs presque tout le mois d'aotit. L'autre espèce n'est pas encore ouverte à la même époque.

Bourgène. C'est un arbrissean très-commun en France, daus les bois. II \{leurit en juin et juillet, et se multiplietrès-facilement de graines. Quand il est en fleurs, il est couvert d'abeilles.

Véronique de Virginic. C'est une très-jolie plante, qui fleurit en juillet et août. Elle vient dans tous les terrains, et se multiplic fort bien de semences.

Salicame. C'est une plante pérenclle, et qui se troure ordinairement en France dans les chaussées : elle fleurit en juillet, ct se multiplie de graines.

KeTniA C'ost un superbe arbrisseau, qui 
donne de très - belles fleurs. Celui à fleurs blanches est sur-tout fréquenté par les abcilles. Il fleurit dans les mois d'août ct de septembre, ce qui rend sa multiplication très-intéressante pour les abeilles, qui ordinairement dans ces mois-là manquent de pâturages. Il se multiplie aisément de graines, et vient en plein vent dans les environs de Paris. J'en ai vu plusieurs qui ont résisté à l'hiver dernier, quoique le plus rude qu'on ait jamais éprouvé en France.

\section{H A P I T R E V.}

Des plantes qui donnent an miel une qualité nuisible aux abeilies, et auxhommes.

IL est difficile de croire qu'il y ait autant de plantes défavorables aux abeilles, que le disent certains auteurs: s'il y en a quelques-unes qui puissent occasionner à leur miel des effets pernicicux, ce seroient, peut-être, nos plantes narcotiques, telles que la jusquiame el autres; mais jusqu'ici l'expérience, du moins dans le Levant, et joserois presque dire, môme dans ces pays-ci, n'en a rien appris. 
sur les abeilles. Liv. I. Chap. V. $3+7$

A Syra nous ne connoissons uniquement que la fleur de scille, (royez le commencement du chapitre premier, lirre premier ) qui produise un miel amer; mais il n'a jamais nui, ni à la santé des abeilles, ni à celle des hommes. Il est rrai que de cette espéce de miel, que les abeilles ramassent dans le mois d'octobre, c'est-à-dire après la récolte des ruches, on ne fait guère d'usage pour les besoins domestiques; on le laisse entièrement à la cliscrétion des abeilles.

M. Buch'oz cite plusieurs fleurs nuisibles aux abeilles, celles de l'orme et du narcisse, du sureau, de l'arroche fétide, dn cornouiller sanguin, de l'auréole des bois. Pour ce qui est du tilleul, il paroît décidé que cet arbre, bicn loin d'être nuisible aux abeilles, leur est au contraire on ne peut plus utile; on peut roirce que nous en arons dit plus hant, d'après M. Duchet dans la liste des plantes fréquentées par les abeilles.

Quant anx autres fleurs, je ne crois pas que leurs mauvaises qualités soient aussićvidemment reconnues, que notre auteur le prétend. Pour sen convancre, il falloit que dans les cantons où ces plantes abondent, et peadant le temps 
de leur floraison, l'on retirât quelque rayon qui contînt du miel ramassé sur ces fleurs et qu'on en fît la décomposition, pour découvrir sill étoit composé de matières différentes des miels ordinaires du même canton, ramassés dans d'autres saisons.

Quoi qu'il en soit des miels de la France et de ceux de l'Archipel, il paroît cependant que le miel peut, dans certains autres pays, avoir quelquefois de manvaises qualités. Nous rapporterons ce que Tournefort nous dit à ce sujet dans ses voyages du Levant, qui contiennent quelques détails assez curieux. Nous commencerons par une courte relation du charmant arbrisseau dit chameerododendron, dont cetauteur nous fait la description.

" Le Chamoerododendron est un arbrisscau qui se trouve dans les environs de Trébisonde, et qui porte des bouquets composés de plus de vingt fleurs de toute beauté. Cette plante aine la terre grasse et humide, et passe pour malfaisante. Les bestiaux u'en mangent que lorsqu'ils ne trouvent pas de meilleures herbes. Les habitans de Trébisonde, par une tradition fort ancienne, fondéc sans doute sur quelques observations, assurent que le miel queles abeilles 
SUR les abeilles. Liv. I. Chap. V. $3+9$ en retirent, étourdit ceux qui en mangent, et cause des nausées. »

Dioscoride a parlé de ce miel à-peu-près dans les mêmes termes. "Autour d'Héraclée du « Pont, dit-il, en certains temps de l'année, le « miel rend insensés ceux qui en'mangent, et c'est « sans doute par la vertu des fleurs d'où il est " tiré: ils suent abondamment; mais on les sou" lage en leur donnant de la rhue des salines et " de l'hydromel à mesure qu'ils vomissent. Ce « miel est âcre et fait éternuer : il efrace les rous" seurs du visage, si on le broie avec du costus, « mêlé avec du sel ou de l’aloès ; il dissipe les " noirceurs que laissent les meurtrissures. Si les " cliens ou les cochons avalent les excrémens des "personnes qqui ont mangé de ce miel, ils tom« bentdans les mêmes accidens. »

"Pline a mieux débronillé l'histoire de ce chamoerododendron dont on vient de parler, que Dioscoride et Aristote. Ce dernier a cru que les abeilles amassoient ce miel sur lc buis ; qu'il rendoit insensés ceux qui en mangeoient, et qui se portoient bien auparavant ; qu'au contraire il guérissoit les insensés. Pline en parle ainsi : « 11 est des années où le miel est très-dangcreux autour d'Héraclée du Pont. Les auteurs n’ont 
" pas connu de quelles fleurs les abeilles le reti" roient. Voici ce que nous en savons. Il y a une "plautedans cesquartiers, appelée cegholelhron, "dont les fleurs, dans les printemps humides, "acquierent une qualité très-dangereuse, lors"qu’ellesse flétrissent. Le miel que les abcilles " en font est plus liquide que l'ordinaire, plus pe" sant et plus rouge. Son odeur fait éternuer: "ceux qui en ont mangé suent horriblement, se " couchent à terre, et ne demandent que dır ra" fraîchissement. " Il ajoute ensuite les mêmes * choses que Dioscoride, dont il semble qu'il ait "traduit les paroles."

"Mais, outre le nom d'agholethron, qui ne se trouve pas dans cet auteur, voici une excellente remarque qui appartient uniquement à Pline. «On tronve, continuc-t-il, sur les mêmes " côtes du Pont, une autre sorte de miel qui est "nommé monomenon, parce qu'il rend insensés " ceux qui en mangent. On croit que les abeilles " l'amassent sur les fleurs du Rododendron, qui "se trouve communément parmi les forêts. Les "peuples de ce quartier-là, quoiqu'ils paient aux "Romains une partic de leur tribut en cire, se "gardent bien de leur donner de lewr miel." "Il semble qu'on peut, d'aprés ces passaģes, dé- 
Sur les abeilles. Liv. I. Chip. V. $3 j$ l terminer les noms des deux espéces de chamœrodendron que nous connoissons. La premic̀re, suivant les apparences, est l'xgholethron de cet auteur; car la seconde qui fait des fleurs purpurines, approche beaucoup plus du rododendron; et l'on pent la nommer rododendron Pontica, pour la distinguer du rododendron ordinaire, qui est notre laurier-rose, connu par Pline sous le nom de rododaphne et nericlum.»

"Il est certain que le laurier-rose ne croît pas sur les côtes du Pont-Euxin. Cette plante aime les pays chauds. On n'en voit guère après avoir passé les Dardanelles; mais elle est fort commune le long des ruisseaux dans les îles de l'Archipel; ainsi ce rododendron du Pont ne sauroit être notre laurier-rose. Il est donc trèsvraisemblable que le chamœrododendron à flcurs purpurines, est le rododendron de Pline."

Je suis d'autant plus persuadé que le laurierrose des îles de l'Archipel n'est pas la plante dont les fleurs produisent le mavais miel de Trébisonde, que ces fleurs ne donnent jamais de miel : nous en avons fait plusieurs expériences à Syra, et j'ai toujours trouvé le fond de ces -fleurs sec, sans la moindre apparence de quel- 
que substance mielleuse; d'ailleurs le tube de ces fleurs est si long, que dans le cas même où il y auroit du miel, l'abeille ne pourroit jamais le sucer avec sa petite trompe.

"Quand l'armée des dix mille approcha de Trébisonde, il lui arriva un accident fort étrange, et qui causa une grande consternation parmiles troupes, suivant le rapport de Xénophon, qui cn étoit un des principaux chefs. »

"Commeil y avoit plusieurs ruches d'abeilles, dit cet auteur, les soldats n'en épargnèrent pas le miel : il leur prit un dévoiement par haut et par bas, suivi de vertiges; de sorte que les moins malades ressembloient à des ivrognes, et les autres à des personnes furieuses et moribondes. On voy oit la terre jonchée de corps comme après une bataille; personnc néannoins n’en mourut, et le mal cessa le lendemain, environ \&̀ la même heure qu'il avoit commencé; de sorte que les soldats se levèrent le troisième jour, dans l'état d'affoiblissement où l'on est après avoir pris une forte médecine."

"Diodore de Sicile rapporte le même fait dans les mêmes circonstances. Il y a toute apparence que ce miel aroit étésucé sur les fleurs de quelqu'une de nos espèces de chamorodendron. 
SUK les abeilles. Liv. I. Chap. V. 353 dendron. Tous les environs de Trébisonde en sont pleins; et le P. Lambert, Missionnaire Théatin, convient que le miel que les abeilles sucent sur un certain arbrisseau de la Colchide ou Mingrélie, est dangereux et fait vomir. It appelle cet arbrisseau oleandro giallo, c'est-àdire, laurier-rose jaune, lequel sans contredic est notre chamarododendron Pontica maxima, mipilifolio, flore luseo. La fleur, dit ce Père, tient le milieu entre l'odeur du musc et celle de la cire jaune. Cette odeur paroît approcher de celle du chevre-feuille, mais est incomparablement plus forte.

«Dans le jardin de M. Le Monnier, premier Médecin du Roi, à Versailles, on cultive deux espèces ci'arbrisseaux, qu'on appelle chamorododendron; cependant leurs fleurs ne donnent presque aucune espèce d'odeur. Cela prouve, ou que ces deux arbrisseaux ne sont pas les mêmes dont parlent tous les auteurs cités cidessus, et dont les fleurs sentent très-fort, ou que ce défaut d'odeur provient du terrain et du climat de la France. D’ailleurs j'ai sourent examiné ces deux arbres, et je n'ai jamais vu aucune abeille sur leurs fleurs, quoique j'y aie,

Tome $I$.

$Z$ 
$35+$ TRAITÉ C OMPLET

observé plusienrs autres espèces d'insectes qui y picoroient.

P.S. Mí. l'Abbé de Lille, dans une de ses Notes sur les Géorgiques de Virgile, dit, en parlant de notre chamorododendron, que c'est un nom bien barbare. Cependant il est

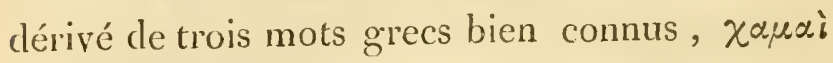
qui signifie humi, par terre; podor, rose, et Sevdpov, arbre, c'est-à-dire, arbre de rose nain, pour la distinguer du laurier-rose qui s'éleve quelquefois à plus de 12 pieds. Si l'on devoit en croire M. l'Abbé de Lille, il faudroit donc en dire autant d'une infinité de mots composés de la langue grecque, dont un des principaux et des plus beaux caractères étoit de se prêter plus qu'aucune autre, à ces sortes de mariages.

Le mot Encyclopédie, par exemple, n'est-il plas égralement composé des trois mots grecs,

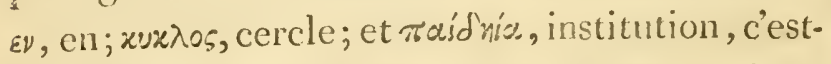
à-dire, instruction en cercle, instruction universelle qui embrasse la totalité des sciences? Tant de savans qui ont mis la main à ce livre si vanté, n’auroient-ils donc travaillé que d'après une d'śnomination barbare? 
sur les abeileles. Liv. I. Chap. VI. 355

\section{H A P I T R E V I.}

Des avantages qu'un État peut retirer de la culture des abeilles.

"L Es abeilles, dit M. La Grenée, sont d'un si " grand produit, qu'il est étonnant que les gens " de la campagne s'en occupent si peu, et que " de tous les sages gouvernemens qui sont en "Europe, à peine en est-il quelqu'un qui pense " à exciter ses sujets à cette culture. Il y a beau" coup de villages où l'on n'en voit point. Ce" pendant, il est certain qu'elles font la richesse " de plusieurs pays, et qu'anciennement la France " en retiroit de grands avantages. Rien ne sou"lageroit plus les gens de la campagne, qui "la plupart sont si souvent pressés, que de s’a* donner à cette occupation qui est fort lucra" tive, et qui exige peu de soins."

En effet, pour décider combien le sentiment de M. La Grenée est juste, il suffit l'observer à quel haut prix se vend le produit des abeilles; 


\section{TRAITÉ COMPLET}

je parledu miel et encore plus delacire; eh! combien ne sont-ils pas nécessaires à l'homme, tant pour le culte religieux, que pour le luxe et les besoins de la vie? Il suffit de faire attention à la quantité de miel et de cire que des ruches soignées donnent chaque année l'une portant l'autre. It est arrivé chez nous dans une bonne année qu'une seule ruche a produit un quintal et plus, de miel, c'est-à-dire I 20 à I 40 livres, et six à sept de cire. Ordinairement elles donnent danslesannées médiocres trenteà quarante livres, et même plus, de miel, et deux ou trois de cire; dans les bonnes années cinquante à soixante, et de la cire à proportion, en comptant pour quinze à vingt livres de miel une livre de cire.

Ajoutez à cela le peu de temps que le propriétaire employe à ses ruches, et le peu de peine que demande leur culture, relativement au gain qu'il en retire. Tout cela suffit pour qu'unjusteappréciateur des choses n'hésite point de donner ì cette culture une préféreace raisonnable sur les autres occupations de la campagne, d'autant plus qu'il n'a pas besoin de consacrer en particuiier ni des terrains ni des arbres pour leur nourriture. Dans ces mêmes champs destinés à foumir le pain aux hommes, et le fourrage 
SUR les Abeilles. Liv. I. Chap. VI. $35 \breve{7}$ aux animaux clomestiques, la diligente abeille trouve à se nourrir, sans rien diminuer de la récolte destinée aux uns ou aux autres. Ces mêmes arbres que le Cléateur à faits pour fournir des fruits savoureux et du bois à l'usage de l'homme, donnent à l'industrieuse abeille de quoi former ses rayons délicieuxqui retournent encore au profit de l'homme. De là vient que nos ancêtres en faisoient un cas si particulier, comme l'attestent leurs écrits, et qu'ils donnoient tous leurs soins à cette culture. Par ce que l'histoire nous en apprend, on voit que les abeilles n'étoient pas ingrates envers leurs maîtres. M. Daniel Wilclman, auteur Anglois dont j'ai sous les yeux l'ourrage sur les abeilles, traduit en Italien arec des notes par Contårdi, cite au quatrième chapitre de ce traité, un auteur moderne qu'il ne nomme point, et qui atteste que lorsque les Romains furent les maîtres de l'île de Corse, ils imposèrent sur ses habitans un tribut en cire, qui se montoit jusques à deux cent mille livres par an. Supposons que l'ile conscrvât encore au moins 200 mille livres de cette matière, voilà donc 400,000 livres de cire effecives. L'on sait que la proportion de la cire au miel est cnviron d'un à 15 , ou 20; au moins c'est ainsi quelle existe

$$
\text { Z iij }
$$


à Syra. Or en multipliant ces 400,000 livres par I 5 ou 20 , on trouve plus de 6 ou 8 millions pesant de miel, indépendamment des 400,000 livres de cire. Quelle richesse ne seroit - ce point pour la Corse, si la culture des abeilles étoit sur le même pied qu'elle étoit alor's, sur-tout aujourd'hui que le prix de la cire et du miel est sans comparaison plus haut qu'il ne l'étoit autrefois?

Puisque j’ai cité l'exemple de la Corse, je dois rapporter ici une observation très-juste sur les avantages que la France peut retirer de la culture des abeilles daus cette île. Elle se trouve dans une brochure anonyme qui a paru il y a quelques années, et qui commence par ces mots : "Quelle Nation! elle va toute seule." "Lî̉le de * Corse, dit l'anteur, peut nourrir des millions " de ruches, d'autant plus précieuses que le cli« mat de l'île rendant les fleurs, et les arbustes * parfaitement aromatiques, il en résulte que le * produit de ces mouches, seroit d'une qualité « très-supérieure à celle des ruches de Pologne « et de Russie. Le miel de Narbonne et celui " de Bretagne suffisent à très-peu de chose près "pour la consommation du royaume; mais in"dépendamment de ce que l'on pourroit vendre 
sur les abeilles. Liv. I. Chap. VI. 359 " à l'étranger le miel de Corse, l'on tireroit " encore de cette île plusieurs millions de cire " brute de la plus belle qualité, que l'on manu * factureroit dans le royaume, tant pour notre " usage, que pour servir à nos exportations cliez " l'étranger. L'on peut se faire un idée de l'é* norme quantité de cire que peut nous fournir " la Corse, quand on saura que dans les temps " où cette île étoit fendataire de la cour dẹ * Rome, elle payoit aux Papes son tribut en " cire, et que la quantité en étoit t-elle, qu'elle * suffisoit à la consommation des Églises de "Rome, et de tout l'état écclésiastique, con"sommation que tous ceux qui ont vu l'Italie * ct qui connoissent le luxe des Églises en lu" minaires savent être immense. " L'auteur conclut: «Voilà donc encore pour cet article! plu" sieurs millions versés annuellement en France; « au lieu d'être portés chez l'étranger » Il ajoute en note: «Le millier de cire, prix moyen, re* vient à peu près à deux mille francs : il s"en " consomme environ deux millions tournois; je "veux croire que moitié seulement se tire de "l'étranger, mais il faut observer que nous " tirons aussi pour des sommes très-considéra" bles, des suifs du Nord, ou de l'Amérique. 
«Or, le bas prix des cires, nous mettroit a "portée de nous passer de res suifs; et l'on " peut dire quil résulteroit de la muliplication "des ruches en Corse, une économie totale "de quatre millions, et que nous pourrions "vendre aux étrangers en cire brute, la con"sommation de nos manufactures décluite ».

A supposer que ce calcul parût un peu éxagééré, ce yure je ne crois pas, l'opinion de l'auteur est juste quant au fond. Or, je conclus que si une culture sage et éclairéc des abeilles, admise dans la seule île de Corse, peut procurer une si grande utilité à l'État, on jourroit cn attendre une bien plus grande, si on établissoit cette culture dans tout le royaume, ou du moins dans ses provinces mériclionales (1). L'introduction de cette culture n'est rien moins qu'impossible, si l'on en juge d'après ce que M. la Grenćc

(1) J'ai lu dans la gazette de France du 2r septembre ${ }_{7} 37$, cet article d'Hanovre, en date du 30 atuut. L'éducation des mouches à miel est un des objets de l'industrie des habitans de cette province; le produit de la cire s'est élevé cette année 1787 , à 300,000 livres pesant. 11 est certain que l'Hanovre ne vaut pas une province de France, pour la température. Quon voie parJà, ce que pourroit espérer la France de cette culture. 
SLR les abeilles. Liv. I. Cha. V'J. 35 i rapporte dans la préface de son traité page 38 : * La France, dit-il, possédoit anciennement, "trois fois autant de propriétaires d'abeilles « qu'elle en a actuellement, qui tous faisoient "de bons profits, malgré leur grand nombre." (r) Si cela est vrai pour lạ France, combien plus pour l'Espagne et les autres pays méridionaux?

Pour confirmer ce que j'ai déjà dit, je veux rapporter une observation que jai souvent faite sur la Turquie. Il semble que le systême politique de cet empire ait été conçu dans la vue de rendre ses habitans les plus misérables et les plus paurres qu'il soit possible. L'agriculture y est négligée et les cultivateurs opprimés,

(1) On élevoit jadis, beaucoup plus d'abeilles en France, dit M. Pingeron, qu'il n'y en a aujourd'hui; c'est ce qui donne lieu à différentes lois et à différens droits seigneuriaux, sur-tout à celui d'aboilage ou abeillage, qui est un droit que les Seigneurs châtelains ont en plusieurs lieux, de prendre seuls les abeilles sui se trouvent dans les forêts de leurs seigneuries. Aboilage se prend aussi pour un droit établi dans plusieurs contumes, cn rertu duquel les Seigneurs peuvent prendre une certainc quantité d'abeilles, decire ou de miel sur les ruches de leurs rassaux. 
saus qu'cn aucun temps et en aucun licu, on ait pensé à leur donner la moindre émulation et à y porfectionner cet art utile. D'un autre côté le commerce est si peu encouragé, si vexé parmi les sujets du Grand-Scigneur, que tous les étrangers le lont plus avantageusement, presque de dix pour cent, que les gens dupays qui payent une donanebeaucoupplus forte que lesétrangers ( I). De plus il existe trés-peu de manufactures en

(1) Voiei ce que dit N. Volnay, rol 2, p. 39o, à ce sujet.

"Presque tont le commerce de Syrie est entre les mains des Francs, des Grees ct des Arméniens : ci devant il ćtoit en celles des Juifs : les Musulmans s'en mêlent peu, non qu'ils cu soient détournés par esprit de religion, ou par nonchalance, comme l'ont cru quelques politiques, mais pare quils y trourent des obstacles par le gouvernement. Fidele ì son esprit, la Porte au licu de donner à zes sujecs une préference marquée, a trouvé plus lucratif de vendre à des étrangers leurs droits et leur infustrie. Quclques États d'Europe, en traitant avec elle, ont obtenu que leurs marchandises ne paicroient de donane que trois pour cent, tandis que celles des sujets Tures, paient de rigueur dix, on de grace sept pour cent. En outre, la douane, une fois acgunitte dans un port, nist plus exigible dans un autre pou des Trancs. Avec tant de ciésarantages, esitil ćton- 
sur les abeilles. Liv. I. Chap. VI. 363 Turquie; et le peu qu'il y en a, à peine a t-il cours dans le pays. Il est permis de faire entrer tout ce que l'on veut dans ces états, ot los étrangers ne payent que trois par cent ou environ. Il faut ajouter à cela que le Grand-Seigneur ne pouvant pas mettre des impositions sur le peuple dans des circonstances urgentes, il faut qu'il s'accumule des trésors en temps de paix, pour être prêt en temps de guerre; de sorte que

nant que les Musulmans cèdent le commerce à leurs. rivaux?"

A cette note de M. Volnay, il faut donner quelque explication. D'abord il se trompe en confondant les sujets du Grand-Seigneur aree les Turcs soi-disant Musulmans, c'est-à-dire, orthodores. Toutes les Nations soumises à l'empire Ottoman, sont des rayas, ce qui veut dire proprement cultivateurs. Au commencement de l'empire des Turcs, les seuls Chrétiens exerçoient ce métier, et les Turcs s'appliquoient à la gnerre. Par le mot raya, le Ture entend encore ce que nous exprimons par le mot sujet. Ainsi les Tures ne sont point censés sujets du Grand-Seigneur, mais les sectateurs de Mahomet, et le peuple choisi de Dieu, dont le GrandSeigneurn'est que le chef. Ainsi on voit pourquoi cenx qui connoissent à fond le gouvernement des Turcs, disent que le Grand-Seigneur ne peut exercer aucun despotisme sur les sectateurs de Mahomet ou du coráa 
le plus fort numéraire de l'état est ordinairement renfermé dans son trésor. Les grands de l'empire initent le souverain, et tiennent leurs trésor's sicachés, qu'il reste à peine quelque numé* raire pour l'usage public. Malgré cela, quoique les labitans de l'empire Ottoman ne soient pas les peuples les plus riches de l'Europe, ils pourroient cependant aller de niveau avec les peuples de la plus grande partie des autres états. Cela

M. de Volnay se trompe encore, lorsqu'il dit que les Tures paient entre sept pour cent par grace, et dix de rigueur; ce. sont les rayas qui paient ainsi; leș Turcs, au moins ceux de Constantinople, de Smirne et de Salonique, c'est-à dire, ceux de l'Europe ct de l'Asie Mineure, ne paient que trois pour cent. Je crois même que ceux-ci ne paient qu'une seule douane, ainsi que les Francs.

Enfin, quoique la loi de Mahomet ne défende pas aux Tur's toute commuaication sociale et commerçante arec lés chrétiens, comme la loi de Mö̈se la défendoit aux Juifs avee les étrangers, il n'en est pas moins vrại que l'espril de la loi de Mahomet, et le fanatisme de la multilude, cmpêchent les Tures de faire un commerce plus élendu avec les sujets chrétiens des puissances étrangères. Les 'Tures en général néprisent ceux de leur nation gui s'expatrient, et qui sćjournent dansles pays ehrétiens: ils les regardent sous une forme d'inficélité 
sur les abeilles. Liv. 1. Chap. VI. 368 ne peut s'attribuer qu'à la bonté, et la fertilité du sol, qui pour peu qu'il soit cultivé, suftit pour maintenir le peuple dans une sorte d'aisance, et sur-tout à la culture des abeilles, dont on s'occupe dans presque toutes les provinces de l'empire, au moins dans les maritimes. On sait l'immense quantité de cire, que les Européens en tirent chaque année de Smyrne, de Salonique, de la Morée, et des autres pays de la mer

et d'apostasie. En outre, la loi du coran ordonne plusieurs cérémonies aux Turcs, comme des prières, des ablutions et des sacrifices, qu'ils ne pourroient observer dans les pays chrétiens où il n'y a ni Imans, ni mosquées. D'ailleurs, pour faire un commerce solide et suivi avec les nations européennes, il faudroit qu'ils s'établissent chez elles avec leurs femmes et leurs enfans; ce qui exposeroit les unes aux yeux de tout le monde, et les autres à la perversion, deux choses capitales que leur loi défend. On voit done que M. Volnay a tort de critiquer les politiques qui ont prétendiu, que c'étoit par esprit de religion, et par nonchalance, que les Turcs abandonnoient aux chrétiens le commerce. Une des raisons qui s'y oppose encore plus, c'est la crainte qu'ils ont des corsaires Maltois ; ils n'osent se hasarder de se'mettre en mer sous leur propre pavillon; et quand ils sont forcés de faire un long voyage, ils sont obligés de le faire sous le pavillon d'une puissance chrétienne. 
blanche; à l'égard de la mer noire, il suffit de lire l'ouvrage que M. Peyssonnel, ancien Consul du Roi, vient de publier sur le commerce des provinces Turques de la mer noir, pour se convaincre de ce que j'avance, il dit à la page r 25. "La cire est l'article le plus important du com«merce de la Moldavie (et de la Valachie); " elle est de très-belle qualité, plus belle encore "que celle de Valachie. On la vend au même prix. $\gg$ Il dit, page $\mathbf{6 2}$, sur le commerce de la Bulgarie. "Il sort de la Bulgarie une quantité "immense de cire, elle est jame et d'excel"lente qualité. On la rend pure. Son prix est " de 38 à $1^{2}$ paras l'ocque, suivant les années. " Le para vaut I sol 6 deniers, l'occa pèse trois livres de Marseille.

Tout cela fait voir la justesse du sentiment de M. La Grenée, rapporté au commencement de ce.chapitre, que "rien ne soulageroit plus "les gens de la campagne, dans les besoinsdont "la plupart sont si souvent pressés, que de "s'adomner à la culture des abeilles."

Dans les îles de l'Archipel, quand un pawve paysan peut parvenir à se former un capital de vingst it trente raches, il assuré d'avoir trouvé le moyen de soulager et de soutenir commo- 
Sur les abeilles. Liv. T. Chap. VI. 367 dément sa famille, en y joignant le peu qu’il peut retirer de ses travaux de la campagne.

Je crois ne pouvoir mieux finir ce chapitre qu'en rapportant ce que M. Mentelle pense at ce sujet, en parlant de l'ile de Cuba en Amćrique, dans son livre intitulé, Choix de, lectures géographiques et hisloriques. Tome 5, part. 2. "Lorsque, dit cét auteur, la Floridefut cédée « en 1763, par la cour d'Espagne, à celle de "Londres, les cinq ou six cents misérables qui "végrétoient dans cette région, se réfugiérent " à Cuba, et y portèrent quelques abeilles. Cet " insecte utile se jetta dans les forêts, s'y établit " dans le creux des vieux arbres, et se mul" tiplia avec une célérité qui ne paroît pas croya"ble. Bientôt la Colonie qui achetoit beaucoup " de cire pour ses solennités religieuses, ei re" cueillit assez pour ce pienx usage et pour "d'autres consommations; elle eut un peu de "superflu en $177^{\circ}$, et sept ans après on en « exporta sept mille cent cinquante quintaux «et demi pour l'Europe ou pour l'Amérique. "Cette production augmentera nécessairement " sous un ciel, et sur un sol qui lui sont éga"lement favorables; dans une île où les ruches * donnent quatre récoltes chaque année et où 
"les essaims se succèdent sans interruption. * A l'appui de ce que M. Mentelle dit de l'île de Cuba, voila ce que je viens de lire dans l'ouvrage de Dom Ulloa intitulé, Mémoires philosophiques, historiques concernant la découverte de l'Amérique, traduit de l'Epagnol. Je ne dois point passer sous silence que les essaims d'abeilles domestiques, se sont beaucoup multipliés à lî̀le de Cuba, dans le voisinage de la Havane, pendant le court espace de temps qui s'est écoulé depuis 1764. après que la paix eut été conclue avec l'Angleterre. II n'y en avoit pasauparavant; car celles qu'on y voyoit étoient sauvages et d'une espèce différente. Les familles qui jusques alors avoient demeuré à St. Augustin de la Floride, s'étant rendues dansl'île de Cuba, après qu’on eut évacué ces lieux, apportèrent avec elles quelques ruches qu'elles placèrent à Guanavacoa et en d'autres endroits, par pure curiosité. Ces mouches se multiplièrent au point qu'il s'en répandit dans les montagnes; et l'on commença à s'appercevoir qu'elles devenoient nuisibles aux cannes à sucre, dont elles se nourrissoient. Leur fécondité fut si grande, qu'unc ruche donnoit un essaim, et quelquefois deux par mois : l'un ordinaire, l'autre moindre en les châtrant 
Sur les abeilles. Liv. I. Chap. VI. 369 châtrant tous les mois : on ue les soignoit même pas avec toute l'attention qu'on y porte en Europe. Elles rendoient autant de miel et de cire, que dans les endroits où l'on n'a soin de les châtrer qu'une ou deux fois par an. La cire est des plus blanches, et le micl aussi clair ê d'aussi bon groût qu'on en puisse trouver. D'après cesfaits, il est évident que la cire et le miel pourroient devenir une des branches les plus arantageuses pour le commerce de cette île, sans même s'occuper très-soigneusement des mouches, ni négliger la canne à sucre, qui scroit toujours l'objet principal. »

D’après ce récit, je ne puis assez m’étonner de ce que dans les îles adjacentes de l'Amérique, et spécialement dans les îles Françoises, on n’ait pas encore admis et propagé la culture des abeilles. Si l'on a cru qu'elles n'y réussiroient pas, il me semble que ce qu'on a raconté de leur prodigieux succès dans l'île de Cuba devroit nous rassurer sur ce point. Si le gouvernement a eu des motifs politiques pour empêcher cette culture dans les colonies de l'Amérique je doịs respecter sa prudence de sacrifier au plus grand bien de la mère-patrie, le prolit des colonies; mais je pense au moins, qu'il est du devoir de Tome $T$. A a 
l'administration de s'appliquer sérieusement à encourager dans toute la France la culture des abeilles pour y procurer une plus grande abondance de cire. Mais sicette faute d'économie rurale vient de la négligence des habitans desîles, je ne puis comprendre comment des hommes qui se sont expatriés pour faire fortune, peuvent négliger un moyen si propre à les enrichir, sur-tout n'ayant presque aucun besoin, pour cette exploitation, de ces malheureux nègres, sans lesquels on ne sait faire venir le sucre ni le café. J'ai demandé à une personne respectable de St. Domingue, pourquoi on y négligeoit la culture des abeilles: elle m'a répondu que c'étoit parce que ces insectes ravageoient les cannes à sucre, et parce que la nudité des Nègres les exposoit à en être incommodés. Dom Ulloa cité ci-dessus, est à la vérité du même sentiment, quant au dégât des cannes à sucre; mais j’avoue ingénument, que j'ignore comment il peut avoir lieu, si les abeilles dérobent le suc mielleux qui peut couler à travers les fentes des cannes à sucre : il me semble que ce n'est pas une perte réelle; ce suc se perd en vapeur, ou il devient la proie d'autres insectes; il seroit donc bien plis avantageux que les abeilles en profitassent. 
Sur les abeilles. Liv. I. Chap. VI. 37 I En supposant qu'une trop grande quantité de ruches occasionnât quelque diminution dans le produit des cannes à sucre, ne retrouveroit-on pas à s'indemniser de cette perte avec usure par la quantité de miel que les abeilles donneroient, et sur-tout par la riche récolte de cire qu'elles fourniroient?

Quant aux nigres qui seroient exposés à la piqûre des abeilles, à cause de leur nudité, je réponds qu'elles n'attaquent personne en pleine campagne, à moins qu'on ne veuille les prendre on les chasser, quand elles voltigent sur les fleurs. Pourquoi d'ailleurs ne s'habilleroient-ils pas pour soigner les ruches? l'utilité que les colons en retireroient en vaudroit bien la peine.

Au reste les nègres de presque toutes les côtes d'Afrique, où se fait la traite de ces malhenreux, savent tres-bien cultiver les abeilles; et la quantité de cire que les Européens en tirent en est la preuve; leur nudité n'empêche donc pas quills ne les approchent, et quils ne les snignent.

II en est qui craignent qu'en multipliant les abeilies dans les îles à sucre, les personnes enployées aux rafineries n’en soient inquiétées. Mais quand ces insectes trouvent de quoi se A a ij 
nourrir dans les campagnes, ils ne vont point dans des maisons pour en tourmenter les habitans. Aussi voyons-nous à Syra, qu'on peut manipulèr le miel dans les maisons sans aucun danger, torsque la campagne est couverte de fleurs. Or, St. Domingue doit, comme l'île de Cuba, fournir aux abeilles, dans toutes les saisons, de quoi les nourrir amplement; il ne scroit donc pas à craindre que les ouvricrs des rafi. neries en fussent inquiétés.

Au surplus, rien de si facile que de l'empêcher : lïle de St. Domingue est assez étendue, pour que l'on pût disposer les choses de manière quc les ruches fussent éloignées dè quelques lieues, et alors les abeilles n'en approcheroient sûrement pas.

Avant de quitter cette matière, il n'est pas inutile de résoudre une difficulté, ou, pour mieux dire, un ancien préjugé. Des gens de la campagne condamnent la culture des abeilles, parce qu'ils sont persuadés que ces insectes muisent aux fruits des arbres. En suçant le miel des fleurs, disent-ils, les abeilles dérangent la fécondation, ce qui occasionne la chûte d'une grande quantité de fruits.

Voici ce que le fameux Linné dit à ce sujet : 
SUR les abeilles. Liv. I. Chap. VI. $3-3$ "Il n’est pas encore décidé, si les abeilles et " autres insectes qui se nourrissent de miel, en "suçant le nectar desfleurs, occasionnent quel"que inconvénient aux petits embryons, ou sils " clérangent la génération. Ainsi, on ne peut " pas expliquer même actuellement, selon les * loix de la nature, ce que Quintilien et Sénèque. * rapportent de ce richard qui infectoit avec le " poison les fleurs de ses arbres, pour tuer toutes "les abeilles d'un pauvre paysan, qui venoient " en voler le miel (I). s"

Malgré le doute de M. Linné sur ce point, je suis très-convaincu, que la succion du miel que les abeilles, ainsi que d'autres insectes, font sur les fleurs des arbres, ne leur porte aucun prějudice, et ne dérange en aucune maniere la fécondation; et voici sur quoi se fonde ma conviction. Dans lîle de Syra il y a peu de pommiers, d'abricotiers, de pêchers et de poiriers :

(a) Utrun verò insecta aliaque mellisuga animalcula sorbendo nectarium è floribus, dunnnum inferant benellis cmbrionibus, nondun evictum est; ideoque iste locus Quintiliani, et Senecre de divite inficiente flores suos veneno, ut pauperis apes mel ejus furantes perirent, vix ac ne vix quidem secundum naturce legem eticumnitm explicari potest, Amæn. acad. t. 6 , diss. I 15, p. 265. 
à peine en trouveroiton cinquante de chaque espèce; d'un autre côté, il y a quantité de ruches: malyré cela, ces arbres sont ordinairement si chargés de fruits, qu'on y en compteroit autant que de feuilles. J'ai encore observé à Versailles dans le jardin de M. Ruffin, sur quelques plantes de bourrache, une grande quantité d'abeilles qui y picoroient depuis le matin jusqu'an suir; et ayant examiné avec attention les calices de ces plantes, je les ai trouvés remplis de graines sans en découvrir un seul vide: d'où je conclus, que si les abeilles en suçant le nectar des fleurs apportoicnt quelque atteinte à la conscrvation des fruits, les arbres fivitiers à Syra n'auroient produit ancun fruit, et nos plantes de bourrache ne seroient pas montées en graines. C'est donc sans fondement que certaines personnes -pensent, que les abeilles nuisent aux fruits des arbres.

P.S. Je viens de lire dans l'Encyclopédie méthodique quelques particularités qui confirment ce que nous venons de dire sur les avantages de la culture des abeilles. "Les ruches, dit M. "l'abbé Tessier, sont, en général, d'un bon pro"duit. Dans certaines années à la vérité, elles « ont peu de miel et de cire, ou donnent peu 
sur les abeilles. Liv. I. Chap. VI. $\quad 375$ " d'essaims, mais elles clédommagent amplemen "le propriétaire dans d'autres années. On cs" time, toute compensation faite, le produit " annuel d'une ruche à six francs; souvent "il monte à dix." (On m'a assuré, que M. le curé de Bonneuil, dans les environs de Paris, retire jusqu'à un louis de chacune de ses ruches. Cela dépend de la manière de les gouverner.) "Quelquefois les fleurs des plantes sont si char* gées de miel et de cire, que les abeilles, qui * en ramassent autant qu'elles en trouvent (c'est" à-dire qu'elle en peuvent emporter) en font " des récoltes étonnantes. J'ai rapporté plus haut "d'après M. Duhamel, que le curé de Tillay " le Pelieux en Bauce, ayant placé une ruche "sur un cuvier renversé, auquel il aroit fait " un trou, en retira cinq à six livres de cire, «et quatre cent vingt livres de miel. Ordinaire"ment les ruches de la Bauce, quand elles sont " bonnes, et qu'elles ont dleux ou trois ans, pèsent " de 80 à 100 livres. En déduisant ${ }^{\circ}$. douze à * quinze livres pour le poids des abeilles, et celui " cle la ruche, faite d'osier, ayant deux pieds " de hauteur, sur un pied et demi de diamètre " dans la plus grande largeur; 2". deux livres " ou deux lifres et demie de cire; le surplus est $\mathrm{A}$ a iv 


\section{3ै76 TRAITÉ COMPLET}

" en miel, et la plus grande partie de belle "qualité. " La proportion de la cire avec le miel est plus grande chez nous, que dans la Bauce; c'cst-à-dire, que d'mne certaine quantité de rayons, qui par cremple produisent roo livres de miel, nous retirons 5 à 6 livires de cire : au Jieu qu'en Bauce, d'aprés le rapl lort de M. l'abbé Tessier, à peine en retire-t-on deux on trois. J'exposerai ailleurs la saison de cette différence.

\section{H A P I T R E VII.}

Canses de la décadence de la cullure des abeilles en France.

S I jai marqué de l'étonnement de ce que les îles de l'Amérique n’imitoient pas l'exemple de colle de Cuba, je dois en dire autant de l'Europe entière. En effet, on ne comprend pas comment toutes les nations de l'Europe, d'uilleurs si intelligentes et si industrienses on ce qui regarde. leur intérêt, négligent la culture des abeilles. On ne voit presque nulle part cette culture en vigueur, et perfectionnéc; partout 
Sur les abeilles. Liv. I. Chap. VI1. 377 au contraire elle est superficielle, imparfaite, et fort au dessous de l'extension dont elle seroit susceptible, et sur-tout en France; caril est certain qu'elle y étoit autrefois beaucoup plus étendue, et qu'il y aroit une plus grande abondance de cire dans le royame. Si on pouvoit démêler la cause de cette décadence, et du découragement où se trourent la plupart des gens de la campagne pour la relever, on pourroit y apporter un remede efficace, et exciter chezeux l'émulation nécessaire pour s'y aclonner.

Quelques anteurs rapportent diverses raisons de ce désordre. M. La Grenée, après avoir dit dans son traité des abeilles, que la France renfermoit anciennement trois tois plus de propriétaires d'abeilles qu'aujourd'hui, cesont ses termes, ajoute: "Que cette extrême diminution est "venue des impôts excessifs dont les gens de "la campayne étoient chargés, et pour le paye"ment desquels les ruches leur ont été en"levées."

A ce propos je me rappelle d'avoir lu clans l'ourrage de M. Necker sur l'administration des finances, que le gouvernement ayant demandé, je ne sais pourquoi, à un intendant de province une liste exacte de toutes les ruches qui se trouroient 
dans son district, les propriétaires de ces ruches informés de cette demande, et effirayés des conséquences qui pouvoient en résulicr, les détruisirent entièrement. Je ne doute done point que M. La Grenée n'ait eu raison d'attribuer la diminution des ruches aux impôts dont les peuples ont été surchargés; mais cette cause n’a pu avoir lieu que dans la circonstance où les tributs étoient exhorbitans, et où la vexation étoit insupportable; d'où il suit, que la décadence de cette culture n'a dû avoir lieu que dans quelques provinces opprimées et pour quelque temps seulement, c'est-ì-clire, autant que la vexation a duré ou existé. Il faut donc quill $y$ ait une autre cause qui ait réduit constamment cetle culture à un état si cléplorable, puisque jusqu'à préscnt personne n'a pu la relever; elle est d'autant plusétonnante, que presque tontes les autres branches de l'économie rurale ont été successivement perfectionnées en France, ou dans les autres parties de l'Europe.

Cette cause, selon moi, vient d'abord de ce que les ruches dont se servent les gens de la campagne, dans presque toute l'Europe, sont défectueuses, soit par la manière dont elles sont construites, soit par leur forme et leur disposition. 
sur les adeilles. Iiv. I. Chap. VII. 379 Il en résulte qu'on ne sait comment s'y prendre pour les bien gouverner et pour les faire prospérer; et cela est si vrai, que M. La Grenée dit, que lorsquon leur témoigne, (aux paysans), de l'étonnement de ce qu'iłs ne s'em'pressent pas d'avoir part au profit qu'ils savent que procurent les abeilles, la plupart allèguent. la difficulté de les gouverner, quand on veut le faire, disent-ils, dans toutes les règles.

Il résulte de cette ignorance une grande perte de ruches tous les ans par de rudes et longs livers, soit à cause des fausses teignes qui en font périr une grande quantité, de sorte qu'il est presque impossible qu'clles passent trois ou quatre ans sans en être attaquées; soit encore parce que leurs propriétaires ne pouvant absolument se servir d'autres moyens, font périr euxmêmes beaucoup de ruches, tous les ans', pour retirer les provisions de ces laborieux insectes. Ces obstacles, ces difficultés, et ces pertes ne peuvent que décourager les cultivateurs, et surtout les gens de la campagne, qui finissent par abandonner cette culture dont nous avons démontré ła grande importance : il y a mêmê des paysans qui en sont si effrayés quils la conclamnent hautement comme inutile, sans aucun 
profit, et ruineuse pour ceux qui s'en occupent. Comment en effet un paurre habitant de la campagne pourroit-il entreprendre une telle culture, et encourager ses voisins par son cxcmple, si, après avoir formé, à force de peines et de fatigues, un fond de vingt outrente ruchesdans l'espérance de l'aider au sontien de sa famille et de payer ses impositions avec leur produit, il s'en voit, à la find'un hiver rude et longr, dépouillé on de la totalité ou de la plus grande partie? Si ce malheureux en a conservé quelques-unes pour completter à la longue celles qu'il a perdues, il voudra en retirer du miel et de la cire, pour subvenir à ses pressans hesoins; alors il met son esprit à la torture pour trouver des moyens faciles et commodes d'enlever la subsistance des abeilles, sans risquer de les faire périr, et sans exposer les ruches à une perte certaine; mais ces pratiques, inventées aree beaucoup de sagacité, sont toutes de nature à les faire périr pendant l'hiver, ainsi que nous le. verrons plus bas dauns les chapitres 14 et 15 . Diallewes ecs procédés destinés à des personnes aisées, et qui ont beancoup de loisir, sont presque impraticables pour les gens de la campagne, parce yu'ils sont coûteux et cmbarrassäns. Ils sont 
sur les abeilles. Liv. I. Chap. VII. 38, même d'une si difficile exécution que, si je me trouvois moi-même dans un semblable cas, ¡’aimerois mieux laisser mes abeilles tranquilles que de risquer leur précieuse existence.

Voilà ce que font les cultivateurs qui ont un attachement particulier pour leurs abeilles; et c'est ce que feroient peut-êtire tous les cultivateurs, si la nécessité adsolue de vivre et de payer leurs impôts, ne les forçoit de vendre leurs ruches à des marchands, qui parcourent les petits villages, et cherchent à tenter le pauvre propriétaire de ruches. Encore si elles étoient achetées par des personnes bien intentionnées qui eussent le projet de les conserver et de les soigner, ce ne seroit pas un si grand mal, parce que la postérité et la multiplication des abeilles u'en souffriroit pas; mais ce sont des marchands qui ne les achetent que pour les faire périr, et pour en retirer le miel et la cire. Je laisse donc à considérer si le paurre habitant de la campagne ne doit pas regarder la culture des ruches avee indifférence; lui qui ne travaille que pour être payé à la fin de la semaine, et qui ne sème que pour recuellir dans son temps; comment pourroit-il se livrer à une culture qui présente tant-de difficultés, expo- 
sée à mille dangers, et où le profit est sincertain?

Il fautsı peu de chose pour dégoûter le pauvre cle former de nouveaux projets d'industrie ! deux ou trois exemples de mauvais succès suffisent dans un village pour épouvanter le reste des habitans, qui se gardent bien de se donner des soins, quandils voyent, pour ainsi dire, l'assurance d'y perdre leur temps et leur argent.

Il est certain que si les gens de la campagne trouvoient assez de profit dans la culture des abeilles, pour que ce profit stable les récompensît avec usure de leur dépense, de leur peine et de leur temps, la culture des abeilles se propageroit avec rapidité en France, et l'abondance si desirée de la cire et du miel s'y introduiroit. L'expérience nous apprend combien l'émulation est naturelle à l'homme en matière d'intérêt, et que ceux qui voyent enrichir leur voisin; cherchent à employer les mêmes moyens pour acquérir la même aisance. Combien de personnes n'auroicnt pas desiré faire le petit commerce des marrons au palais royal, quand elles ont vu que celniqquien avoit eu lidée y avoit gagné quelque argent? Si done un paysan voyoit son voisin avec un moyen facile de les conserver, se former un nombre de quinze ou vingt ruches, et en tirer 
sur les abeilles. Lir. I. Chap. VII. 383 assez de profit pour entretenir sa famille et payer ses impôts, il ne manqueroit certainement pas d'imiter son exemple; il établiroit bientôt des ruchers, et la culture s'en étendroit de proche en proche.

Je crois avoir indiqué assez clairement la vraie cause de la décadence des abeilles en France, et ce qui l'a réduite à un état de lanģueur si déplorable qu'elle n'a pu s'en relever Les gens de la campagne sont précisément ceux que celte ${ }^{2}$ culture devroit concerner, et ils sont seuls en état de la rétablir. Leur découragement provient donc d'une infinité de peines ct de difficultés que l'on rencontre dans l'économie des abeilles, et sur-tout dans cell es de retirer le miel et la cire des ruches, sans les exposer à une perte presque assurée; enfin il provient de l'impossibilité où l'on est d'empêcher ces pertes totales et subites qui désolent le cultivateur. Voilà àpeu-près la véritable cause de la décadence de la culture des abeilles en France. Voyons à présent le remède qu'on pourroit y apporter. 


\section{H A P I T R E VIII.}

Moyen efficace de relever la culnure des abcilles.

L. E sentiment des auteurs sur la décadence de la culture des abeilles n'étant pas le'même, il doit $y$ avoir aussi de la différence dlans les moyens quon peut proposer pour y remédier. Il seroit trop long de détailler ici cesopinions et ces moyens. Je me contenterai de rapporter ceux qui ont été présentés par M. La Grenée, et j’exposerai ce que je pense moi-même à cet égard; mais avant tout, jobserverai que l'Empereur régnant, dont les principales vues sont de faire fleurirle commerce daus ses états, et de lávoriser les cultures dans tous les genres, voulant cuconrager celie des abeilles, afin de la propager avec plus de facilité et derapidité, et alin d'agrandir le commerce de ses sujets, a pris un'parti digne cle sa ģénérosité viaimentroyale. Cáa été de promettre un florin pour chaque ruche qu'un proprictaire cultiveroit; et afin 
SUR les A BeIlles. Liv. I. Chap. VIII. 385 que personne ne fit périr ses ruches dans l'automne pour en retirer le miel et la cire, il régla que le florin ne se payeroit qu'au commencement du printemps, temps auquel personne ne trouveroit de profit à les faire périr pour en retirer les provisions.

M. La Grenée, dans la préface de son traité, page 35 , après avoir déploré la décadence de la culture des abeilles en France, et la grande disette de cire que ce royaume éprouve, ce qui l'oblige à la tirer des pays étrangers pour satisfaire à ses besoins, propose deux moyens pour relever la première, et procurer l'abondance de l'autre. « Je crois, dit-il, ne me point tromper, " en disant que j’exige pour cela deux choses; la " première, qu'on multiplie dans le royaume le " nombre de gens qui s'occupent à gouverner « les abeilles, et qu'on leur enseigne la manière « la plus simple de le faire. Tant qu’il n'y en « aura pas plus qu'on n'en voit actuellement, ce " peu de gens auront beau multiplier chez eux «leurs paniers d'abeilles et les gouverner, " comme nous venons de voir, jamais on ne « verra venir l'ancienne abondance; mais en mul" tipliant considérablement les propriétaires des " abeilles, la quantité de ruches, et par conséTome $I$. 
"quent la cire et le miel, deviendra immense " dans le royaume, et cela sans gêner en aucune «manière les propriétaires, puisque chacun « n'en aura que ce que son jardin, ses fonds, * ou ses occupations lui permettront d'en avoir. "Je conviens qu'il faut pour cela une espèce " de miracle; mais les Souverains peuvent "l'opérer en peu de temps, en accordant de "légères exemptions aux uns, et quelques mar"ques de distinction aux autres, telles à-peu"près que les épis d'or dont Henri IV grati"fioit ceux de ses sujets qui, de son temps, se "distinguoient dans l'art cle l'agriculture."

"La seconde chose que je demande, c'est que " ces propriétaires fassent périr tous les ans une " certaine quantité de ruches. Ils seront assurés "d'y trouver une quantité de miel et de cire, « souvent considérable, et toujours capable de « leur procurer un profit certain et actuel, à "moins que les annćes ne soient absolument " mauvaises. Je le répète donc afin qu'on y fasse «attention. Le défaut d'abondance de cire et « de miel dans le royaume vient, non de ce qu'on "fait périr tous les ans une certaine quantitó " de ruches, puisqu’il seroit nécessaire d'en faire " périr bien davantage pour procurer l'abon- 
sur les abeilles. Liv. I. Chap. VIII. 387 "dance souhaitée, mais de ce que, faute d'er« couragemerit, il n'y a pas à beaucoup près assez "de personnes qui s'occupent du soin de gou"verner les abeilles, et que la plus grande partie "de ceux qui s'y adonnent, s'en acquitte mal."

Dans ce paragraphe de M. La Grenée, il y a des observations très-intéressantes; cependant le second moyen qu'il propose pour propager la culture des abeilles, et pour procurer parlà l'abondance de la cire dans le royaume, est contraire au systême qu'a embrassé l'Empereur, de ne donner la récompense du florin qu'au printemps, pour empêcher que les propriétaires ne fassent périr leurs abeilles. Au reste, dans le chapitre 13 et suivans, je dirai ce que je pense sur ce moyen. Ceux dont se sert l'Empereur, et celui que l'auteur propose, à l'exemple de Henri IV, sont bons pour exciter le commun du peuple à embrasser la culture desabeilles; mais ils ne suffisent pas pour établir dans le royaume une influence solide, constante et universelle, la seule capable de produire en France l'abondance de cire et de miel qu'on desire.

En effet, à quoi sert de multiplier le nombre des propriétaires, et que ceux-ci, à force de peines et de dépenses, augmentent le nombre de leurs

$\mathrm{Bb}$ ij 
ruclses, si, faute de les bien gouverner, de connoître tous leurs besoins, de savoir et de pouvoir remédier à leurs accidens, un hiver un peu rigoureux et long, et d'autres causes font périr la plus grande partie de ces ruches, pour ne pas dire toutes, chez les pauvres gens de la campagne? A quoi bon multipler nos ruches, si, faute d'un moyen simple et facile de retirer le profit de nos peines, sans risquer la vie des abeilles, et sans exposer les ruches ainsi récoltées à une perte évidente pendant les hivers suivans, nous sommes forcés à les faire périr volontairement, pour avoir leurs provisions? Pourra-t-on compter sur un nombre quelconque de ruches? Pourra-t-on espérer une abondance constante de cire dans le royaune, si on ne change pas la manière de gouverner les abeilles, telle qu'elle se pratique communément en France?

Puisque nous avons établi clans le chapitre précédent, comme le dit fort bien M. La Grenée lui-même, que la cause principale de la décadence de la culture des abeilles provenoit du découragement des habitans de la campagne, qui les empêchoit de s'adonner à cette culitrre; et puisque nous avons prouvé que ce découragement n'avoit d'autre cause que les diffi- 
Sur les abeilles. Liv. I. Chaf. VIII. 389 cultés qu'on éprouve à bien gouverner les abeilles, et l'ignorance où l'on est généralement sur ce point, d'où il résulte une grande mortalité , comme nous l'avons dit; il s'ensuit, par une conséquence naturelle, que le moyen principal, ou plutôt unique d'exciter les gens de la campagne à s'adonner sérieusement à la culture des abeilles, est de leur présenter une sorte de ruches, qui soit peu coûteuse, qui leur offre une méthode facile, simple et commode, de gouverner les abeilles, et qui embrasse toutes les parties de leur économie; de sorte que les pauvres cultivateurs pouvant compter, autant qu'il est possible, sur la vie et sur la conservation de leurs ruches par le moyen de cette méthode nouvelle, puissent aussi espérer avcc probabilité de voir récompenser leurs soins et leurs peines par leur produit.

Comme la difficulté de récolter et de retirer le miel et la cire obligge plusieurs cultivateurs à détruire les ruches dans bien des endroits, nous avons observé que cette difficulté contribue encore à dégoûter les paysans, et s’oppose, geénéralementparlant, à la prospérité des abeilles: il faudroit donc qu'un nouveau systême d'économie fournit un moyen facile et simple de 
récolter les ruçhes sans perdre beancoup d'abeilles, et sans coûter beaucoup de peine aux proprićtaires, etl'objet sera rempli; car prétendre plus, c'est-à-dire, de ne perdre aucune abeille, et de ne pas être tant soit peu incommodé par ces insectes, ce seroit vouloir piller une ville et emporter les biens des citoyens, sans coup férir, ni d'une part, ni de l'autre: on sent que je parle d'une ville bien fortifiée, et dont les liabitans seroient bien pourvus de toutes sortes d'arme pour leur défense.

Sur-tout nous avons conclu dans le chapitre ci-dessus, que la grande mortalité qui arrive subitement dans les ruches, et qui emporte souvent les ruchers les plus peuplés, est la cause principale qui décourage communément les cultivateurs de s'adonner à cette culturc. Or il faut donc que la nouvelle méthode nous offie un moyen facile et simple de découvrir la cause de cette mortalité, et d'y remédier; de sorte que, si on ne pouvoit pas en prévenir tontes les pertes, on pût du moins éviter ces destructions générales de vingt et trenteruches tout à la fois; car il ne faut pas s'attendre à trouver un moyeu d'empêcher qu'il n'en périsse quelquefois, puisque ce seroit rendre icsabeilles immortelles, 
sur les abeilles. Liv. I. Chap. VIII. 39i

Telle est la nouvelle méthode de gouverner les abeilles, que je propose au public dans le présent ouvrage. Elle embrasse tout ce que nous avons dit ci-dessus, et d'autres observations importantes pour ceux qui voudroient s'appliquer sérieusement à la culture des abeilles, et en tirer un profit certain et constant. Quiconque lira avec attention, et sans prévention, tout ce que j'expliquerai dans les chapitres et les traités suivans, se convaincra évidenment que je tiens tout ce que je promets. Quoique je sois très-persuadé de cette vérité, par l'expérience que j’en ai faite, et par une application particulière de quinze annéés, inclépendamment de l'étude quej'ai faite des meilleurs auteur's et cultivateurs; cependant avant de donner ce traité au public, je veux le soumettre au jugement de personnes qui, par leur talent et leurs lumières sur cet ubjet, peuvent former un jugement judicieux sur le mérite ou sur le démérite de tout ce que je propose et de ce que j'avance, étant très-résolu de ne pas laisser voir le jour à mon ouvrage, si les personnes éclairées ne lui trouvoient pas la justesse et l'utilité que je lui suppose.

Je commencerai donc par examiner les causes $\mathrm{Bb}$ iv 
de toutes les espèces de mortalités des abeilles. Quand j’aurai découvert cette cause principale, je chercherai à y remédier de la manière la plus efficace. Ensuite je discuterai avec la plus grande impartialité quelle est la manière la plus utile pour les propriétaires et la plus avantageuse pour les abeilles; si c'est celle des anciens qui tailloient et qui châtroient leurs ruches sans détruire les abeilles, ou celle de plusieurs modernes, qui les font périr pour en retirer les rayons; ensuite, j'entrerai dans le traité de la culture des abeilles. Je commencerai par les ruches, après quoi je traiterai de suite ce qui regarde ces insectes. On verra par la simplicité et la facilité de tous les moyens que je proposerai, que la méthode que je prescris est à la portée de tous les hommes en général, et qu’elle est par conséquent le moyen le plus puissant de multiplier la culture des abeilles, de la relever de sa décadence, et de produire l'abondance de cire et de miel si désirée dans presque toute l'Europe, et sur-tout en France.

Cependant pour produire ces bons effets avec plus de rapidité dans le royaume, il faut que le gouvernement s'en occupe, pour faciliter aux pauvres de la campagne les moyens d'entre- 
Sur les abeilles. Liv. I. Chap. VIII. 393 prendre cette culture; il faut que les seigneurs, les curés, les fermiers, et autres cítoyens aisés, animés du bien public et de leur propre intérêt, s'appliquent à instruire leurs vassaux, et leurs paroissiens, sur la véritable méthode de bien gouverner les abeilles; il faut qu'ils leurs fournissent les moyens d'entreprendre et de concluire une telle culture : mais rien sans doute ne pourroit lui être plus avantageux, et en accélérer plus rapidement les progrès, que quelque loi portée en sa faveur par l'Assemblée nationale. L'objet est d'une assez grande importance, pour qu'elle s'occupe quelques momens d'un insecte bienfaisant qui paroît avoir été chargé d'une manière spéciale par la providence de travailler pour le bonheur de l'homme, tout en lui donnant des leçons d'économie, d'industrie et de la plus sage subordination.

Au reste nous aurons occasion de revenir sur les moyens de perfectionner et d'accélérẹ la culture des abeilles en France, dans le chapitre qui terminera cet ourrage. 


\section{H A P I T R E I X.}

Gauses de la morralite des abcilles.

I l y a deux sortes de mortalités qui occasionnent la destruction de leur espèce, l'me involontaire, qui dérive de causes indépendantes de notre volonté; l'autre volontaire, que lcs propriétaires occasionnent en voulant retirer le miel et la cire : je parlerai de la premiere dans ce chapitre, et je traiterai dans le suivant de la manière de l'éviter. Ensuite je discuterai la seconde espèce de mortalité que les propriétaires causent eux-mêmes à leurs ruches.

La cause principale qui produit shez nous la mortalité, et par conséquent la destruction des abeilles, provient des mauvaises années, sur-tout quand clles se succèdent deux ou trois de suite; alors les ruches s'affuiblissent considérablement, et ces pauvres insectes sout exposés à toutessortes d'accidens. D'ailleur's lorsgu'une ruche est affoiblic jusq: 
SUR LES ABEILles: Liv. I. Chap. IX. 39,5 elle est bientôt attaquée des fausses teignes, qui sont une des plus grandes causes de la perte des abeilles. Il est vrai qu'avec nos ruches on peut s'appcrcevoir facilement quand quelqu'une d'elles est attaquée des vers, et alors nous venous bientôt à leur secours, comme je le d1rai ail!eurs. Mais si les mauvaises années se succèdent, et si les vers attaquent de nouveau les ruches déja affuiblies, il faut bien qu'elles succombent.

Cette rermine, dans certaines anuées, emporte chez nous plusieurs ruches; et si elle fait quelquefois tant de ravage dans l'Archipel, où les ruches sont de terre cuite, et par conséquent moins sujettes à en être attaquées, combien ne doit-elle pas être nuisible en France, où les ruches sont communément de paille ou de roseau, et où les vers se nichent avec d'autant plus de facilité, que les ruches ne peuvent se nettoyer, ni si souvent, ni aussi bien que les nôtres? Aussi en résulte-t-il qu'en France elles ne durent pas long-temps, parce que ces vers ne les laissent jamais vieillir. A la véritć. les hivers, quand ils sont rudes et longs, emportent une si grande quantité de ruches,

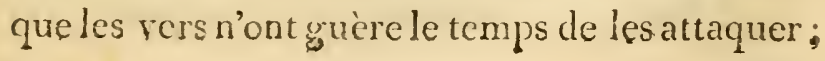


de l'aveu de tous les auteurs, ce qui n'empêche pourtant pas qu'ils ne causent un grand dommage dans les ruches.

Un malheur, plus fâcheux encore que les autres, et qui dégoûte de cette culture, c'est la destruction quelquefois subite et générale des ruches. Des propriétaires qui, à la fin de l'automne, se trouvoient avec trente ou quarante ruches, se sont vus au commencement du printemps avec deux ou trois. Une paurre femme du village de Viroflay, à qui nous ẹn avons vu jusqu'à quinze, nous a assuré que, dans le courant d'un seul hiver, elles les avoit toutes perdues. M. Varpy nous a certifié que dans un hiver il en avoit perdu trente, et qu'il ne lui en étoit resté qu'une scule; M. Ducarne, que dans un hiver, il avoit perdu la moitié des sicunes, et que d'autres particuliers en avoient perdu les trois quarts: il cite un jeune homme dont le plaisir consistoit à élever des abeilles, et qui, après avoir rassemblé jusqu’à vingt-neuf ruches, ne s'en étoit trouvé que deux à la lin liun hiver rigoureux.

La cause de ces grandes mortalités mériteroit d'être recherchée, et il ne seroit peut-être pas difficile de la trouver, et dy apporter remierle. 
SUR les abeilles. Liv. I. Chap. IX. 397

Les gens de la campagne sont persuadés que le froid et les grandes gelées produisent la perte de leurs ruches. Je ne déciderai pas seul cette question, n'ayant aucune expérience sur les pays septentrionaux. Je m'en rapporterai pour cela à ce que disent les auteurs d'après leur propre expérience. Mais ce que M. La Grenée dit à cet égard, me paroît d'une telle évidence, que je ne puis croire que le froid seul puisse faire tant cle ravage. Voici ses propres termes, chap. 1o, $\$ 8$.

" J'ai d'abord cru, comme bien d'autres, que le froid pouvoit causer du dommage à une ruche; mais les froids excessifs qui règnent en Pologne et en Russie (pays si féconds en abeilles, que des forêts entières en sont remplies ), sont une preuve sans réplique du contraire; ainsi il est certain que quoiqu'une abeille seule ne puisse supporter un très-petit degré de froid, réunie à un nombre suffisant de ses compagnes, elle échappe aux effets du froid le plus aigu. J'ai eu effectivement des ruches ouvertes par le bas, qui ont passé, sans en souffrir, l'hiver de 1767 , qui a presque égalé celui de $1709 . "$

Cependant nous conviendrons que le froid, et les gelées, lorsqu'elles sont excessives, peu- 
vent quelquefois faire périr une ruche, par la négligence de quelques propriétaires. Les abeilles, dans des ruches mal couvertes, doivent perdre leur chaleur naturelle; mais ce qui est difficile à croire, quoique cependant très-vrai,c'est que la chaleur en fait peut-être périr plus que le froid. Nous avons lu quelque part, notamment dans l'ouvrage de M. Ducirne, qu'il a perdı, dans une année, plusieurs ruches par la grande ehaleur. Et M. Contardi rapporte que dans la provinee d'Artois ( dont le climat néanmoins n'est pas des plus chauds), la chaleur y fit périr une fois vingt-quatre ruches. Cela doitd'autant plus nous étonner, que quoiqu'clle soit certainement beaucoup plusforte à Syra que dans ce pays-ci, nous n'avons jamais entendu dire qu'elle y ait produit cet effet. Nous arons su seulement qu'un cultivateur, après avoir mis dans un sac un essaim qu'il avoit trouvé, l'avoit perdu pour l'avoir attaché et suspendu à un arbre; mais le soleil qui dardoit sur l'essaim, en fit crever toutes les abeilles. Il n'y a pas longtemps que nous avons vu périr aussi, par la chaleur, plus des trois quarts des abeilles d'un essaim qu'on avoit renfermé dans une boîte d'acajou, faite en Angleterre, d'après la méthode de 
SUR les abeilles. Liv. I. Chap. IX. 399 M. Wildman, en les transportant du grand Montreuil à Versailles.

Il peut arriver encore que la chaleur fasse périr une ruche, parce que les rayons trop ramollis peuvent tomber et écraser la reine; et alors cette perte doit entraîner celle de toute la ruche, comme nous le verrons ailleurs. Mais revenons aux causes de la mortalité.

Quelques auteurs prétendent que le défaut ou la corruption de l'air, et le manque de nourriture, sont les principales causes qui font périr tant de ruches en hiver.

M. La Grenée dit à ce sujet, " que la disette de vivres, et le défaut d'un air pur, sont les deux fléaux les plus funestes aux abeilles. La méthode de ne construire que de foibles ruches, et de ne leur laisser que de petites entrées, en fait périr tous les ans un grand nombre.» C'est aussi le sentiment de $M$. Ducarne, et de tous ceux qui ont des connoissances dans cette partie.

Il ne reste donc qu'à examiner celle qui con. tribue le plus à ces deux causes, ou le défaut d'air, c'est-à-dire l'infection, ou la disette des vivres. Pour y parvenir, il n'y a qu’à observer dans quel état se trouvent les ruches après la mort totale des abeilles; car si on y trouve des 
rayons avec des provisions de miel ou de molividhe, on ne peut plus douter que ce ne soit ou une infection pestilentielle, ou un froid extrême, faute d'aroir couvert les ruches, ou enfin quelque autre cause semblable qui les ait fait périr. Mais si, après la perte des abeilles, on ne trouve dans les rayons ni miel, ni molividhe, c'est une preuve que les abeilles sont mortes de faim. Il est encore certain que lorsqu'on les trouve mortes dans les cellules des rayons, c'est qu'elles s'y sont introduites pour chercher leur nourriture, et que n'y en trouvant pas, elles ont péri.

Ayant demandé en effet à plusieurs personnes qui se plaignoient d'avoir perdu beaucoup de ruches pendant l'hiver, en quel état étoient ces ruches après la perte des abeilles; elles mont répondu qu'elles n’y avoient rien trouvé qui pût servir à leur nourriture, et que ces insectes, en grande partie, avoient péri dans leurs cellules ou sur les tablettes: d'où l'on peut conclure que la faim seule avoit occasionné cette perte, et que la disette en étoit la principale cause : c'est ce qui doit arriver fréquemment aux environs de Paris, et dans les pays sous la même température, où l'on élève les abeilles de la même manicre.

J'ai 
SUR les abeilles. Liv. I. Chap. IX. 40 I J'ai dit que la disette de vivres causoit la grande perte des ruches aux environs de Paris, et dans les autres provinces septentrionales; car au midi de la France, je suis persuadé qu'il n’y a guère que les vers qui les détruisent. Nous en avons l'expérience à Syra, où, malgré toutes les attentions que nos cultivateurs ont de nettoyer leurs ruches, pour les préserver de ce fléau, ils ne peuvent les empêcher quelquefois d'y succomber. Eh! quel avantage n'ont pas ces cruels ennemis, lorsque les ruches ne sont que de paille ou d'osier, qu'on n'en peut voir l'intérieur, et qu'on ne les nettoie qu'une fois par an?

Cette différence entre la cause de la mortalité des ruches dans les pays chauds et dans les pays froids, provient de ce que, dans les premiers, les abeilles produisent en général plus de miel que dans les autres. Les hivers y sont plus courts, et par conséquent leur nourriture plus abondante et plus sûre.

Dans le nord au contraire, les fleurs fournissent moins de miel aux abeilles : les hivers étant plus longs, il en résulte que les ruches n'y sont pas aussi bien garnies de provisions que dans les pays méridionaux; que les abeilles en consomment beaucoup plus dans un long 
402 TRAITE COMPLE T

espace de temps, et qu'elles sont par conséquent plus exposées à en manquer.

On dira peut-être que dans les pays chauds, les abcilles consomment plus de miel que dans les autres où elles restent assoupies pendant une bonne partie de l'hiver.

Cela est vrai pour la Rassie, la Suede, le Danemark et autres pays du nord; mais aux environs de Paris, et dans les parties septentrionales de la France, où il y a tant de belles journées pendant l'hiver, les abeilles s'agitent, sortent, et rentrent contiuuellement aux rayons d'un beau soleil, et elles doivent consommer sans donte alors une plus grande quantité de miel.

Il faut que nous remarquions ici que les abeilles au commencement du printemps, périssent quelquefois de disette au milien de leurs provisions : alors elles consomment ce qu'elles ont, pour former leur première couvée, et pour la nourrir; s'il survient ensuite du mauvais temps, et quild dure quelques jours, elles ne penvent plus sortir pour s'approvisionner, et elles périssent avec leur couvain, au milieu de l'abondance qui les environne.

Mais si le défaut de vivres est la première cause de la mortalité des abeilles en hiver, 
Sur les abeil.tes. Liv. I. Chap. X. 403 pourquoi MM. Là Grenée, Ducarne et de Blangis, avancent-ils que de sonlever les ruches dans le froid, et de leur donner une circulation d'air libre, c'est un moven efficace de les en préserver? C'est ce que nous expliquerons dans les chapitres suivans.

II y a d'autres causes qui occasionnent souvent la perte des abeilles, comme le butin que les fortes enlèvent aux foibles; la mort de la reine, etc.; mais nous en parlerons ailleurs.

\section{I A P I T R E X.}

AIanière d'éviter les mortalités dont on vient de parler.

A y ant exposé, dans le chapitre précédent, les principales canses de la mortalité des abeilles, il convient de parler dẹ moyens à employer pour les éviter; mais comme, dans le cours du présent ouvrage, nous derons traiter des différentes manières de les sccourir avec facilité dans lemrs divers besoins; nous nous bornerons

$\mathrm{C}$ c ij 


\section{TRAITÉ COMPLET}

ici à indiquer les moyens les plus sûrs de rémédier à ce qui occasionne leur mort ou leur destruction.

Quant à la mortalité qui provient des mauvaisešannées, lorsqu'il en arrive plusieurs de suite, comme elle dépend d'une cause supérieure aux forces humaines, il n'y a d'autre remede que la patience et la constance. Tout cultivateur doit, autant que le lui permettront ses facultés, soutenir avec du miel le plus de ruches qu'il pourra; il sera un jour bien dédommagé de cette dépense.

A l'égard des dégâts occasionnés par les vers, qui exercent particulièrement leurs ravages sur lesruches de paille et de roseau, je ne vois pas de moyen plus efficace et plus simple, que l'usage des ruches dont je donnerai la description dans le livre suivant: leur forme et leur disposition faciliteront les moyens de les nettoyer sans nuire aux abeilles.

Les essaims les plus foibles pourront se garantir des vers dans cette nouvelle espèce de ruches, bien plus facilement que dans les autres; et les fausses teignes ne pouvant pas s'y cacher, seront trop exposées à la poursuite des abeilles, pour pouvoir s'y multiplier. 
SUR les ABEILlles. Liv. I. Chap. ג. 405

Ces nouvelles ruches doivent l'emporter sur toutes celles qui ont été inventées par les auteurs modernes, puisqu'on pourra en visiter l'intérieur, pour connoître les besoins des abeilles, et savoir si elles sont attaquées des vers: on en fera connoître tous les détails, lorsqu'on parlera des moyens de les secourir, et de l'instrument qu'il faut employer. Pour se convaincre que la construction et la forme de nos ruches ont un grand avantage sur celles dont on se sert communément pour les garantir de leurs ennemis, il suffit d'observer qu'elles durent à Syra quinze, vingt ans, et même davantağe, au lieu qu'en France trois ans suffisent quelquefois pour les voir entièrement détruites.

Il a pu arriver que des amateurs curieux et très-expérimentés, comme M. Ducarne et autres, soient parrenus à les conserver quelques années de plus; mais ce n'a été qu'en leur donnant les plus grands soins, soutcnus par une certaine aisance, qu'on trouve rarement chez les gens de la campagne.

On pourra placer et disposer nos ruches avec autant de facilité que les autres; et même en les construisant dans des murailles, comme nous l'expliquerons ailleurs, nous ferons yoir quelles 
406 TRAITÉ COM P L T

seront plus en état de supporter la rigueur du firoid en hiver, et l'ardeur du soleil en été, que toutes celles.qu'on a employées en France jusqu'a ce jour. Voyez sur cela le chapitre suivant, et les chapitres I, 6, 7 et 8 dur deuxième Traité.

Q liant au défaut d'air ou à l'infection, auxquels plusicurs persomes attribuent la perte des ruches en hiver, jaurois beaucoup de peine à y croire. S'ils pouvoient contribuer à leur destruction, ce devroit ètre plutôt dans une autre saison: car en été, il y a sans comparaison plus d'abeilles dans leis ruches quen hiver, et alors tous les corps, et même les abeilles exhalent davantage. Il se trouve d'ailleurs en été plus de matières hétérog̀enes propres à la fermentation et ̇̀ l'exhalaison, comme le miel et la molividhe. 1 est de fait encore que si une épidémie est occasionnée par un air infect, elle est plus dangereuse dans les grandes chaleurs, et qu'elle cesse même à l'arrivéc des froids.

On objectera peut-être qu'en été les abeilles sortent sonvent, prennent l'air, et sont moins sujettes à l'infection. Mais plusieurs d'entre elles ne sortent pas tous les jours, et bien des personues croient que la reine ne sort jamais ; ainsi, 
SUR les abeilles. Liv. I. Chap. X. $40 T$ sil est vrai que l'infection s'introduice dans les ruches en hiver, la sortie des abeilles pendant l'été ne doit pas les empêcher d'y succomber, et leur perte doit être égale dans les deux saisons.

Quoi quil en soit, il est certain que la disposition horizontale de nos ruches garantira les abeilles de linfection d'un air corrompu; et on ne peut avoir cet avantage dans celles qui sont faites en cloche ou de touteautre forme, qui étant fermées de tous les côtés, n’ont qu'une petite ouverture dans leur partie inférieure. La raison en est simple; l'air corrompu, ou toute autre vapeur particulière monte toujours, et lorsqu'il ne trouve point d'ouverture dans la partie supérieure des ruches pour s'échapper, il doit y croupir, et détruire les abeilles.

Nos ruches au contraire, au moyen des trons que nous disposons autour du couvercle, et de ceux que norns ménageons dans sa partie supérieure, sont à l'abri de cet inconvénient : l'air entre, sort et se renouvelle par ces ouvertures; le mouvement des abeilles, le moindre battement de leurs ailes, produit encore dans la ruche une circulation d'air, qui en classe celui qui est corrompu, et y en introduit un 
plus salubre. Eh ! qui sait si la nature n’a pas donné aux abeilles, pour leur conservation, cet instinct qui les porte de concert át exercer ce battement? Au reste on sentira que les ruches, dans toute autre disposition, ne peuvent présenter le même avantage. Et qu'on ne dise pas qu'on pourroit pratiquer les mêmes ouvertures dans des ruches d'une autre forme! Ces trous devant être nécessairement très-petits, pour éviter beaucoup d'inconvéniens, les abeilles les fermeroient bientôt avec de la propolis.

Il nousreste à parler maintenant du défaut de nourriture qui fait périr ordinairement tant d'abeilles en hiver. Pour éviter cette perte dans le Levant, nous observons de temps en temps si les ruches, sur-tout les plus foibles, sont bien pourvues; quand elles ne le sont pas, nous leur donnons du miel mêlé arec un peu d'cau, ou d'autre matière. Quant au moycn que nous employons pour connoître si une ruche manque de vivres, on verra quill est très-simple, ainsi que la manière de fournir aux abcilles leur nécessaire, et sans qu'elles soient exposées à aucun pillage. 


\section{H A P I T'R E XI.}

De la manière de gouvernerles abeilles pendant l'hiver, de les empêcher de consommer leur provision, et d'éviter ainsi leur destmuction.

$\mathrm{E}_{\mathrm{N}}$ Mésopotamie, et dans plusieurs autres provinces de l'Empire Ottoman, les propriétaires qui élèvent des abeilles, ont soin de les tenir tout l'hiver dans des lieux obscurs, et éloignés de tout bruit; ils ne laissent pénétrer aucune lumière dans l'endroit où elles sont renfermées. On les conserve, par ce moyen, dans le meilleur état, et les ruches se trouvent bien fournies au printemps de tout ce qu'il leur faut pour commencer leurs premières pontes, et pour nourrir leurs premiers couvains.

Sans avoir eu dessein de suivre cette pratique, voici ce qui m'est arrivé dans l'Archipel, et ce qui confirme l'exccllence de la méthode dont nous renons de parler. Nous avions mis deux essaims d'abeilles dans deux différens endroits. Nous en avions placé un près de la 
ville, et l'autre à deux lieues de distance. Dans lacrainte des gû̂pes fort abondantes cette anuéelà, nous les bouchâmes tout autour, et pour donner de l'air aux abeilles, nous ne laissâmes qu'un ou deux petits trous daus la partie supérieure, mais par lesquels elles ne pouvoient pas sortir : nous couvrîmes ensuite le devant de la ruche avec de grandes pierres, pour qu'il n'y entrât pas le moindre rayon de lumière. Nous oubliâmes totalement cet essaim jusqu'au milieu de février, c'est-à-dire, pendant quatre mois. Nous le rappelant alors, nous le crûmes péri; nous courûmes le visiter; maisnous le trouvâmes en très-bon état, bien fourni de miel et d'abeilles, et beaucoup mieux enfin que celui que nous avions tenu près de la ville, qui étoit cependant d'égale force, et auquel nous avions fourni du miel à denx reprises. Nous croyons n’avoir trouvé que douze à quinze abeilles de mortes. Cetteruche prospéra davantage, et bien plus rapidement que l'autre.

MM. Ducarne et La Grenée, très-instruits l'un et l'autre, et versés dans l'art de gouverner les abeilles, après avoir souffert de grandes pertes dans des hivers longs et rigourcux, et sur-tout M. Ducarne, invitent les cultivateurs à don- 
sur les abeilles. Liv. I. Chap. XI. 411 ner aux ruches, dans les grands froids, le plus d'air qu'on pourra. M. Ducarne, après avoir raconté, dans son vingt-huitième entretien du deuxième volume, les grandes pertes qu'il a faites pendant les derniès hivers; après avoir ditquedans un de ces hivers, de quatre-vingtruches qu'il possédoit, il ne lui en étoit resté qu'environ cinquante, et que, dans ce nombre, il y en aroit même le quart le mauvaises, assure positivement, que dans les trente ou quarante perdues au contraire, il y en avoit au moins les trois quarts et demi de fortes, et sans contredit les meilleures de toutes.

Après ce récit, il cléclare que l'unique moyen qu'il a imagriné pour prérenir une destruction pareille, c'est de leur domer beaucoup d'air pendant l'hiver.

"Diriez-vous, continuc-t-il, que le moyen dont je me sers pour cela, est précisément le contraire de ce que rous croyez quon devroit faire? Au lieu de les sceller exactement, comme vous le feriez pour les garantir du grand froid, je les y expose tout-à-fait, en élevant les ruches de 4 ou 5 lignes tout autour au dessus de la planche sur laquelle on les pose, c'est-à-dire au dessus du siége. " 
M. Lagrenée dit aussi, page 38 de son petit ouvrage sur les abeilles : "Une seconde pratique (1), qui pourra encore n'être pas dú goût de quelques-uns de ceux dont nous examinons ici les sentimens, c'est celle de laisser les ruches ouvertes tout autour par le bas, l'hiver comme l'été. Voici les raisons de la pratique que nous adoptons, 'de laisser les ruches ouvertes tout autour par le bas............ Elle empêche la trop prompte consommation des provisions d'une. ruche, par le froid que ces ouvertures y laissent entrer; car il est prouvé que plus la chaleur intérieure d'une ruche est considérable, plus la consommation des provisions est grande. “

Tous ces faits, ainsi que la pratique des peuples de la Mésopotamie et des autres peuples du Levant, prouvent qu'il n'y a qu'une manière de gouverner les abeilles pendant les temps les plus rigoureux. Elle consisteà les fixer dans une obscurité parfaite, et à l'abri du bruit

(1) Il avoit déja parlé d'une première qu’il adoptoit, savoir, de faire périr, tous les ans, un certain nombre de ruches, pour s'approprier les protisions des abeilles, comme nous te verrons dans.les chapitres suivans. 
SuR les abeilles. Liv. I. Chap. XI. 413 le plus léger, en faisant passer dans les ruches une certaine quantité d'air, qui conserve autant qu'il sera possible, un degré de fraîcheur presque égal pendant toứt le temps de l'hiver. Il est hors de doute que les ruches ainsi tenues dans le mauvais temps, se trouveront, au commencement du printemps, saines et sauves, bien fournies de provisions, et en état de commencer leur travail. La raison en est sensible; c'est que les abeilles, au milieu de cette obscurité et de cette tranquillité, étant engourdies par le froid, se plongent dans un assoupissement quiles met dans le cas de n'avoir besoin de rien; et alors, plus elles restent dans cet état, moins elles consomment de provisions.

Nous ne craignons point d'avancer qu'une ruche forte et bien peuplée, qui pourroit à peine vivre avec douze ou quinze livres de miel, d'après la méthode ordinaire qui laisse aux abeilles la liberté de sortir et de rentrer dans les belles journées d'hiver, n'en consommeroit certainement pas plus de cinq ou six livres, si elle étoit gouvernée comme nous venons de l'expliquer.

Tout cela se trouve confirmé par M. Ducarne, qui assure avoir eu des ruches très- 
fortes, qui, dans un hiver très-ligoureux, avoient à peine dépensé deux livres de miel.

L'utilité de notre méthode de gouverner les abeilles pendant l'hiver, est constatée par l'expérience de tous les pays du nord, tels que la Suc̀de, la Russie, la Poloune, etc. où ellesrésistent très - bien aux froids les plus excessils. Les sauvages mêmes qui vivent dans le creux des arbres, au milieu des forêts, n'y succrumbent pas, s’il en faut croire des personnes dignes de foi. On ne voit point dans ces pays septentrionaux la destruction des ruches qui désolent les cultivateurs en France, et qui n'y provient ordinairement que du défaut de nourriture. Dans le nord, le degré violent du froid et sa continuité tiennent les abeilles dans l'engourdissement, et les empêchent d'achever leur provision pendant l'hiver, quelque long qu'il soit; de sorte que celle qu'elles ne consomment pas dans les mauvais temps, clles la retrouvent au commencement de la belle saison, et échappent ainsi au fléau de la disette. En France au contraire où le climat est variable et moins rigicle, et où les belles journées sont plus fréquentes, Jes mouches ne restent pas si long temps assoupies; elles 
Sur les abeilles. Liv. I. Chap. XI. 415 consomment lcurs provisions sans mesure et sans économic; elles en manquent bientôt, et elles périssent.

Voilà ce qui constitue la différence de la culture des abeilles des pays septentrionaux, avec celle des pays du midi, et même de cenx qui sont situés entre eux, tels que la plupart des provinces de France.

Dans le nord, cette méthode n’est donc pas nécessaire pour ménager aux abeilles leur nourriture; elles n'ont besoin que d'être renfermées, pour éviter la rigneur extrême des hivers.

Dansles pays du midi, comme ils ne sont pas de longue clurée, et que les campagnes peuvent fournir long-temps de la pâture aux abeilles, la disette s'y fait sentir rarement, et elles n'y sont pas si fréquemment exposćes; mais elles éprouvent d'autres accidens qui ne leur sont guère moins funestes, lorsqu'elles sont libres de sortir pendant l'hiver. Le tort que leur fait cette liberté est inconcevable. Le froid et les vents en font périr un si grand nombre, qu'il n’en reste pas quelquefois le tiers au printems. La méthode de les renfermer pendant quelques mois d'hiver, telle que jela prescris, ne peut donc être que trèsutile. Je ne parle pas des pays de la zone torride, 

416
TRAIT É
COMPLET

et de ceux où l'hiver ne se fait jamais sentir aux abeilles qui y travaillent toute l'année, ainsi que nous l'avons dit ailleurs, en parlant de celles de Cuba.

Dans les contrées où la campagne leur refuse toute espèce de nourriture pendant cinq, six et sept mois, mais où les froids ne sont pas assez constamment rigoureux pour les tenir pendant ce temps dans l'assoupissement, ma méthode doit être employée.

Il n'est pas possible d'expliquer autrement son utilité, que par l'assoupissement où elles doivent tomber quand le froid les saisit.

Dans cet assoupissement qu'on ne peut pas contester, l'inaction et le repos des abeilles doivent les mettre dans le cas de n'avoir besoin d'aucun aliment; ou si elles en prennent, ce ne peut être que très.rarement et en petite quantité. On ne doit pas s'étonner que dans leur inaction, clles consomment très-peu de vivres, puisque telle est la nature et la constitution de tout animal, et même de l'homme.

Mais que cet engourdissement puisse se supposer chez les abeilles, on le prouve par l'exemple de plusicurs autres insectes qui passent tout J'hiver dans cette léthargie, sans rien prendre et 
sur les abeilles. Liv. I. Chap. XI. 417 les fourmis, au rapport de Géer, dans ses Mémoires sur les insectes, dise. 3, p. 5 , t. 2.

"Les fourmis, dit-il, qui vivent, de même que les abeilles, en compagnie, ont été dans la réputation de faire des provisions de vivres pour l'hiver, mais e'est une erreur : elles ne mangent point pendant l'hiver; elles n'ont pas même alors besoin de prendre de la nourriture, parce que le froid les tient comme engourdies. C'est en été que les foumis travaillent à cher. cher des alimens, tant pour elles-mêmes que pour leurs larves, incapables de s'en pourvoic elles-mêmes: les fourmis sont done toujours des insectes très-laborieux de plus d'une façon, quoiqu'elles ne se fassent point de provisions de vivres pour l'hiver, et le sage a toujours raison de renyoyer le paresseux à la fourmi.»

Lopinion de M. Géer est d'autant plus probable, que les provisions des fourmis ne peuvent pas se conserver pendant l'hiver. Nous avons observéplusieurs fois dans le Levant, qu'elles exposoient leurs grains à l'air, à la suite d'une grosse pluie d'été, pour les sécher et les préserver de la pourriture; ce que ne pouvant faire l'hiver, on doit en conclure que pendant celte saison elles ne prennent aucune nourriture, et restent dans l'engourdissement.

Tome $I$.

$D d$ 
M. Ducarne ne reconnoît d'autre avantage à soulever les ruches, et ḋ leur donner de l'air pendant l'hiver, que celui d'empêcher l'infection d'en gagner l'intéricur : cela est d'autant plus vrai, qu'il n'attribue en génćral la mortalité des abeilles, pendant les long's hivers, qu'à l'humidité et à l'infection qui les attaque. Cependant jai fait voir dans le chapitre où nous avons traité de la cause qui en fait périr un si grand nombre en France, que cela n'avoit pas lieu; que l'infection, sur-tout pendant les froids de l'hiver, étoit ce qui entroit le moins dans les causes de leur perte, et que la disette de vivres en étoit la principale et presque la seule.

On demandera sans doute pourquoi les ruches les plus fortes et les plus peuplées, sont quelquefois les plus exposćes à périr, puisqu'une ruche forte et bien peuplée est ordinairement mieux fournie en provisions, qu'une ruche foible en population : c'est qu'une provision de miel, si forte qu'elle soit, devient insuffisante ell égard à cette population. D'ailleurs, l'air d'une forte ruche, libre et exposée à recevoir toutes les impressions d'un air froid et les chaleurs d'un beau soleil, étant plus tempéré , à cause de la grande quantité d'abeilles qu'elle renferme, ces abeilles 
Sur les abeilles. Liv. I. Chap. XI. 4 I 9 sont plus souvent en mouvement, moins sujettes à l'assoupissement, et consomment une plus grande quantité de provisions. Les ruches moins peuplées et plusfoibles, se trouvant au contraire dans un air plus froid, qui les oblige à se res. serrer et à fáre moins d'exercice, consomment moins. Leur foiblesse les rend plus timides, les empêche de sortir de leur ruche à la moindre apparence de beau temps; au lieu queles ruches fortes sont plus vigoureuses, plus alertes et plus hardies, à proportion de leur population. Elles bravent tous les dangers; elles sortent et rentrent aux premiers rayons du soleil; elles doivent donc consommer davantage : aussi cette population, qui sembloit devoir produire le meilleur effet pour le bien - être des essaims, et pour l'avantage des propriétaires, se trouve souvent opérer la ruine des uns et des autres, et cela par la liberté pernicieuse accordée aux abeilles pendant les mauvais temps d'hiver.

C'est ce que nous avons pu proposer de mieux aux cultivateurs de ces insectes, pour arrêter le ravage qui met si souvent la désolation dans leurs ruches, et qquileur occasionne tant de pertes et de chagrins. S'ils font usage de cette pratique, ils en retireront de grands avantages.

$$
\text { D d ij }
$$


Nons devrions expliquer ici la manière la plus propre d'exécuter tout ce que nous venons de dire sur la méthode de renfermer les ruches; mais, pour que les lecteurs puissent mieux saisir ce que nous proposerons à ce sujet, nous n'en parlerons quaprès avoir traité de ce qui regarde nos ruches, et de l'usage qu'on peut en faire en France.

Nous nous sommes suffisamment étendus sur la mortalité accidentelle des abeilles, c'est-ddire, sur cellé que des causes indépendantes de notre volonté peuvent leur occasionner, et sur la manière d'y remédier. A l'égard de celle que plusieurs propriétaires, guidés par de faux raisomemens, leur procurent souvent par une avidité mal-entendue, et en s'appropriant leursprovisions, nous en parlerons dansle chapitre suivant.

Une particularité que nous venons de lire dans l'encyclopédie méthodique, confirme ce que l'on a avancé au commencement de ce chapitre; c'est d'éviter toute sorte de bruit autour des zuchers où les abeilles sont renfermées pendant l'hiver. "Les bords des chemins fréquentés ne conviement pas pour y placer un rucher, parce que les mouches éprouveroient un ébranlement qui les réveilleroit trop tôt de l'engourdişe- 
SUR les AbeIlles. Liv. I. Chap. XII. 42 I ment où elles doivent être pendant cette saison. »

Ce réveil en effet seroit funeste aux abeilles, puisqu'illeur feroit consommer une plus grande quantité de vivres.

\section{H A P I T R E XII.}

Est-il plus avantageux de faire périr les abeilles pour retiver des nuches le miel et la cire, on de se servir, pour y parvenir, de lont autre moyen? Délail de ce qu'on a f'uil jusqu'ci présent.

L a question la plus importante et la plus sérieuse de toutes celles qui sont relatives au gouvernement des abeilles, celle qui mérite le plus Pattention des amateurs, c'est de savoir ce qui vaut mieux, ou de faire périr tous les ans les abeilles d'une certaine quantité de ruches pour en-tirer le miel ou la cire, ou d'en faire la récolte sans les détruire.

Tous les modernes, suivant M.La Grenée, sont de ce dernier sentiment; ils fulminent contre l'usage contraire, qui, selon eux, a mis la cherté et la disette dans tous les pays où on l'a suivi: ils le traitent de cruel et de barbare; ils regar-

D d iij 
dent comme des assassins publics ceux qui le suivent. La manière dont $\mathrm{M}$. Ducarne attaque ceux qui sont dans l'usage de les faire périr, est remarquable et mérite d'être rapportée. Voici ses termes, page 29 de la seconde partie. "On a déjà "fait, avec raison, les portraits les plus affreux " de ces destructeurs; on implore contre eux "le secours et la rigueur des lois; on les cite " dans tous les tribunaux; on rappelle'même " avec complaisance une loi d'un grand-duc de "Toscanc, qui défend, sous des peines très"rigoureuses, de faire périr violemment les "abeilles; on forme des vœux pour que cette " ordonnance soit renouvellée de nos jours, et "maintenue par-tont avec rigueur. » On ne pourroit parler avec plus d'énergie contre des tyrans, contre les oppresscurs de l'humanité.

La manière dont se sont conduits à cet égard les anciens et les modernes jusqu’à présent, est détaillée par M. La Grence qui en donne un extrait si instructif que je ne puis m'empêcher de le rapporter. " Caton le censeur, dit-il, qui " a écrit sur les matières rurales, et qui est mort " 148 ans avant Jesus-Christ, ne parle point des "abcilles; ainsi il paroît que de son temps "elles n'étoient pas mises au rang des profits 
sur les abeilles. Liv. I. Chap. XII. 423 «champêtres, ou que la modicité de leur pro"duit les lui a fait passer sous silence."

"Le savant Varron, qui écrivoit sur les mêmes "matières 123 ans après Caton, est le premier " dont les écrits parlent d'abeilles, et, ce qui " nous intéresse davantage pour le moment, " sur la manière qui étoit en usage de son temps " de récolter le miel. Il dit qu’il y avoit deux "façons de procéder; la première, en changeant " les mouches de paniers; et la seconde, en les " dégraissant. VTarı. lib. cap. I6."

"A l'égard du détail de ces opérations, il « n’en donne aucun : il parle seulement de "quelyues précautions quil est nécessaire de "prendre dans ces occasions, savoir; pour les "abeilles qu'on transvase, de frotter la nou"relle ruche avec de la mélisse, parce qu'elle "les attire, et de mettre en declans, près de "l'entréc, quelques rayons de miel, de peur " que si elles venoient à s'appercevoir qu'il n'y " en eût point, elles ne regrettassent leur an" cienne demeure."

"Et pour celles que l’on dégraisse, que le "sentiment de plusieurs est quion doit leur re" tirer les neuf dixièmes de leü miel, parce que "si on le leur retiroit en entier, elles aban- 
$42+$ TRAITt́ COMPLET-

"cionneroicut leurs moles; quil est d'autres "persones qui est iment qu'on leur en doit laisser "davantage. "

"Ces méthodes au reste ne rapportoient pas "vraiscmblablement beaucoup de fruit, car de « son temps le miel étoit fort rare, puisque l'on " «'on faisoit usaye que dans les sacrifices, "et que l'on n’en seivoit que sur les tables des "riches au premier et au second services."

"Columelle, qui vivoit l'an de Jésus-Christ « 43, ne parle point de changement des abeilles " en paniers, mais seulenent du dégraissage. " sur lequel il s'étend plus que Varron. Mais "il fumt avouer, de bonne foi, que ce quil en " dit est fort obscur, pour un lecteur qui "réfléchit stor les difficultés et les inconvéniens " considérables qui résultent des opérations qu’il "propose."

"Au reste ces deux auteurs ne parlent point "de la méthodo de faire périr les abeilles pour "avoir leur miel; e’est une preuve quielle n’étoit "point eyeore en usigge de leur temps, autre"ment ils l'atroient fait en bien ou eu mal."

"Lit mérhude de transvaser les abeilles, pa" lồt donc avoir été la première inventée pour "récolter le miel; car pour la cire, on la re- 
Sur les abeilles. Liy. I. Chap. XII. 425 " gardoit anciennement comme un objet de peu " de valeur, qui n’étoit cependant pas à négli"ger.... fruclus quamvis aris exigzii, non la"men emittendus est. Colum. 1. 9. cap. 16. Mais "les grand inconvéniens auxquels cette manière "de récolter est sujette, et principalement celui * dont parle Varron, d'être une occasion aux « abeilles d'abandonner la nouvelle ruche si, ce " qui n'y remédie guère, on n'y apporte les « précautions dont nous avons parlé plus haut; " ces inconvéniens, disons-nous, nous firent subs* tituer, avec le temps, la méthode des les dé* graisser. Pour y parvenir on inventa les ruches "dont parle Columelle, qui souvroient par " derriere et par dessus."

"Enfin cette seconde méthode ne répondant « point encore à l'autente de certains proprié" taires, par le peu de fruit qu’elle rapportoit, " on imagina, pour dernière ressource, de faire "périr les ruches dont on rouloit s'approprier "les provisions."

"Commeles usages bons ou mauyais, une fois "reçus, ont toujours des partisans qui les per" pétuent de génération en génération, ces trois "manières de récolter ont toujours subsisté "jusqu’à présent. C'eux qui s'en tiennent aux 


\section{TR A T T E C O P L E T}

« deux premières, ne le font vraiscmblablement « que par conmisćration pour ces charmans "insectes, auxquels ils croient devoir sacrifier * leurs intérêts; mais ceux qui adoptent la der"nière, ne la tiennent certainement, que parce "qu'ils la regardent comme un moyen sûr d’en "tirer plus de profit tant en miel qu'en cire."

Si ceux qui sont d'avis de conserver les abeilles n'avoient d'autres motifs pour appuyer leurs raisommemens que leur compassion pour ces in. sectes, il est certain qu'ils se rendroient ridicules aux yeux des personnes raisonnables; car si cette compassion avoit lieu pour les abcilles, si elle suffisoit pour condamner la coutume de les faire périr, comment justifieroit-on celle de tuer les boufs, les moutons, et les autres animaux domestiques, qui, après avoir rendu les plus errands services, sont sacrifiés pour nous servir d'aliment, ou pour notre prodit? LC Maître Suprême de l'univer's a crécé tout ce qui est sur la terre pour les besoins de l'homme; il a sonmis tous les animaux à sou domane : ainsi il faut céder à l'ordre établi, et croire que ceux qui insistent pour la conservation des abeilles, se fondent sur d'autres motifs plus solides que cette compassion. 
sur les abeilles. Liv. I. Chap. XII. 427

La manière de récolter le miel des abeilles en les détruisant, est encore en usage dans plusieurs parties de la France, en Italie et ailleurs. Ranconi, dans son dictionnaire sur l'agriculture italienne, dit que cet usage est abusif, en ce qu'il tend à détmuire l'espèce de ces animaux si intéressans par leur ingénieuse industrie.

Contardi, dans sa $32^{\mathrm{e}}$ note, dit aussi que cet usage de tuer les abeilles tous les deux ou trois ans, vient de ce que les ruches de son pays sont trop petites : on les fait ainsi, parce quon est dans lintention au bout de quelques années deles détruire pour s'approprier leurs provisions.

Malgré l'autorité de tous les̉ auteurs, M. La Grenée qui le dernier a écrit en France sur cette matière, se déclare avec beaucoup de force pour la méthode de faire périr tous les ans une partie des ruches:

Les raisons sur lesquelles il fonde son opinion se réduisent à deux chefs; l'un, le plus grand profit des propriétaires; l'autre, le plus grand avantage pour la prospérité de l'espèce. Je rapporterai les propres termes de l'anteur, ce qui occupera deux chapitres; mais jespère que le lecteur me le pardonnera, en faveur d'une discussion qui forme la base de l'économie des 
abeilles, et d'où dépend leur bun gronvernement.

Je dois citer d'autant plus volontiers tout ce que dit M. La Grenée à cette occasion, que ses raisonnemens nême démoutreront la nécéssité d'adopter notre forme de ruches: on verra que notre maniere de les disposer doit nous fournir des moyens faciles pour en tirer leurs provisions; nous serons convaincus aussi de la nécessité de faire hiverner toutes les ruches, et sur-tout celles que l'on dégraisse, pour les préserver de la disette. Je préviens que l'examen desmotifs des deux parties, et particulierement de ceux de M. La Grenée, est plus important à discuter qu'on ne le croiroit peut-être au premier apperç̧.

\section{H.A P I T R E XIII.}

Morifs en faveur du systême de faire périr les abeilles pour tirer leuts provisiour., fontes

sur ce que celte pratiquc est de la plus grande ulilité anx Propriétaircs.

" JE m’attens bien, c’est ainsi que M. La Grenée entre en question, que plusicurs pratiques que 
SUR les abeilles. Liv. I. Chap. IV. 429 jadopte dans cet ouvrage, ne seront pas du goût des auteurs dont je parle, et particulicrement celle de faire périr tous les ans une certaine quantité de paniers, pour en retirer le miel et la cire. »

"Mais que ces personnes ne s'imaginent pas que si jai adopté cette manière de récolter, ce soit parce que je suis prévenu contre les raisons qui pourroient me la faire rejetter. Non, j’ai lu scrupuleusement tous les écrits que $j$ ’a pu avoir qui en traitent; je les ai pesés avec attention; j'ai pu en outre essayer de mettre en pratique la plupart des conseils que l'on y donne; mais ni livres, ni expérience n'ont pu me persuader que les manières d'opérer que l'on y expose, fussent préférables à celles pour lesquelles je me déclare.

"Voici les raisons sur lesquelles je me fonde. De quoi est-il finalement question entre ceux qui agitent cette matière? cest de procurer au royaume une abondance de cire et de miel qui n'y est pas; et au propriétaire d'abeilles, un bénéfice actuel et*suffisant pour l'engager à faire les avances et à se donner les peines nécessaires pour se pourvoir d'abeilles et les gouverner." 
"Pour parvenir à ces deux fins, on s'est imaginé qu’il ne s’agissoit que de persuader à ceux qui ont des abeilles, de n'en jamais faire périr, et de se contenter, ou de les changer de panier, ou de partager avec elles leurs provisions; cc quon appelle chatrer ou dégraisser.»

" Mais comme on a éprouvé qu'il n’est ni aisé, ui utile de mettre ces deux méthodes en usage, il n'est pas croyable quels efforts on at faits depuis quelques années, pour trouver des moyens propres à récolter le micl et la cire, sans faire périr les mouches.»

"Je vais donc montrer, le plus brièvement qu'il me scra possible, que ces auteurs se trompent beaucoup, s'ils espèrent, par les pratiques qu'ils ont inventées, satisfaire également le public par l'abondance, et le particulicr par un profit raisomnable. »

* Séduit par l'attrait de la nouveauté, qui que ce soit n'a pensé à prendre en main la cause que je défens : c'est pourquoi, sans cesser d'ĉtre court, comme je viens de le promettre, je le ferai valoir de mon mieux. J'entre en maticre. "

"Pour changer les mouches de panier, il faut s'y prendre deux ou trois jours au plus après la sortic du premier essaim, afin qu'elles aient 
SUR les abeilles. Liv. I. Chap. XIII. 43 I le temps de s'approvisionner dans leur nouvelle demeure. Or quelle quantité de miel trouverat-on alor's, même dans les plus fortes ruches? dix ou douze livres, peut-être moins: Peut-on la comparer à ce qu'on y eût trouvé à la fin d'août, lorsque la provision est faite pour l'hiver, laquelle monte à trente, quarante, cinquante livres, et quelquefois plus? »

"Si vous me répondez qu'au moins vous avez conservé les mouches; je veux bien en convenir, au cas que l'opération ait réussi, ( car il ne faut pas se dissimuler qu'elle manque souvent, et qu'il est trè-ordinaire, sur dix ruches ainsi transvasées, d'en voir périr huit ou neuf') mais il faut aussi que vous avouiez que rous avez fait périr un second essaim prêt à partir, et peut-être le couvain d'un troisième, et que ces deux essaims réunis auroient fait une ruche surement meilleure, que celle que vont composer vos abeilles de ménage. Mais calculons le produit de cette méthode, et comparons-le avec celui de celle que jadopte. Posons pour cela qu'un propriétaire ait dix ruches. Selon votre systême, elles lui fourniront douze livres de miel chacune, ce qui fait cent vingt livres; et il lui restera vingt ruches, dix vieilles et dix 
432 T R A I T É C O M P L E T jeunes: c'est-à-dire, qu’il aura recueilli pour 60 francs de miel, en l'évaluant à dix sols la livre, et qu'il aura pour 200 francs de ruches à 10 francs pièce, ce qui fait en total 200 francs.

«Selon ma méthode, dix ruches lui donneront trente livies de miel chacune, ce qui fait 300 livres; et il aura douze jemnes ruches, savoir, dix composées des premier's essaims, et deux des seconds et troisièmes: c'est-à-dire, qu'il aura recueilli pour 150 francs de miel, et qu'il lui restera pour 20 francs de ruches; ce quifait en total 270 francs. On s’appercoit sans doute que c'est pour faciliter le calcul, que je suppose que les ruclies donnent tous les ans chacune un essaim. „

"Quoique ces deux sortes de profit, tant en miel qu'en ruches, évalué ainsi en arğent, semblent revenir, pour les deux proprićtaires, à peu de chose près à la même somme, il faut y remarquer une différence considérable; car dans la première supposition, le public ne profite que de 120 livres de micl pour sa consommation, et le propriétaire n’a touché que 60 franes d'argent; au lieu que daus la seconde, le public a ell 300 livres de miel, et le proprićtaire à cmboursé x 50 francs."

«Et qu'on ne vienne pas dire que dans les anncés 
SUR les Abeilles. Liv. I. Chap. XIII. 433 années suivantes, le bénéfice du premier propriétaire, par la grande multiplication de ses ruches, passera de beaucoup celui du second; ce seroit dissimuler volontairement et en pure perte, les mortalités fréquentes et presque générales qu'occasionne cette méthode de changer les abeilles de paniers, lesquelles réduiront indubitablement tous les ans le grand nombre de ruches du premier propriétaire à une quantité au dessous de celle demeurée à son confrère; de sorte que l'on peut être assuré que les bénéfices annuels du premier, loin d'être plus considérables que ceux du second, seront toujours beancoup inférieurs. »

"Cette méthode de changer les riches de paniers, après la sortie du premier essaim, n'est donc d'aucun avantage ni pour le public, ni pour le propriétaire. Nous allons voir maintenant si ceux qui se contentent de les châtrer ou dégraisser ont mieux rencontré. "

«Il y a deux manières de le faire : l'une, pour les ruclies qui sont construites en forme de cloches, telles que celles dont on se sert aux environs de Paris et ailleurs; l'autre, pour les abeilles qui habitent des ruches de bois ou de paille. Ces ruches de nouvelle invention, sont

Tome I.

E e 


\section{$43+$ TKAITÉ C OMPLET}

composées de plusieurs étages ou hausses, sans fond, ou avec des fonds percés, pour qu'il y ait communication de l'un à l'autre. Il est de fait que les mouches commencent toujours par remplir l'étage ou hausse de dessus, où on les a d'abord mises, et qu'ensuite el! es descendent dans celui de dessous pour continuer leur travail. Quand on vent récolter, on leur ôte un ou deux étages supérieurs, lorsqu'ils sont pleins, en coupant les communications avec un laiton, comme on le fait pour couper le beurre en motte; puis on couvre l'ouverture de la hausse restante, en sorte quion ôte facilement aux mouches leurs provisions, sans, dit-on, en quelque sorte, qu'elles s'en apperçoivent. »

«Quant aux ruches faites en forme de cloches, tout le monde sait que le magazin à miel étant situé principalement dans le fond de la ruche, les trois traverses, absolument nécessaires pour soutenir l'ouvrage des mouches, sont un obstacle insurmontable à l'extraction des rayons qui le conticnnent."

"Proposera-t-on d'ôter ces traverses? ce ne peut être qu'arcc des tenailles; et pour cela, il faut manier la ruche à son aise, et la tourner en différens sens. Que fera-t-on si les bouts de 
SlR les abeilles. Liv. I. Chay. XIII. 435 ces traverses ne donnent pas de prise? Ensuite il faut les remettre dans leur place. Or, qui ne sent que cette opération ne peut sc faire sans agiter horriblement les abeilles, au point qu'il n'en réchapperoit pas une? la moitić périroit par les piqûres qu'elles n'épargneroient pas à celui qui les tourmenteroit ainsi, en dépit de toutes les fumigations, et le reste par le miel qui les englueroit. Je me souviendrai toujours d'avoir cu une peine extrême à retirer les rayons chargés de miel d'une ruche, dont les abeilles avoient été étouffées, par la seule raison que les traverses n'en aroient pu être retirćes. Que seroit-ce donc si l'on étoit troublé dans son opération par quinze ou vingt mille mouches qui toutes portent l'épée, et se défendent en désespérées? Enfin pour peu qu'on ait d'habitude avec les abeilles, il est aisé de voir que cette manière de récolter est impraticable et infructueuse. "

"Pour ce qui est des abeilles qui habitent des ruches de bois ou de paille à divers étages ou hausses amovibles, comme plusieurs auteurs conseilient d'en avoir, j'accorde volontiers que la récolte s'y fait plus facilement que dans les autres. Mais le propriétaire est-il maître de ne retirer de ces ruches que la quantité de proviE e ij 
sions qu'il juge convenable pour son avantage et celui de ses mouclies? non assurément. Car si les hausses sont minces, et si, dans la crainte de préjudicier aux abeilles, il ne leur retire que peu de provisions, sera-ce son bénéfice? Si les hausses sont épaisses, et que la supéricure contienne tout ou presque tout le miel, en la supprimant, il ne laissera que peu ou point de provisions aux mouches, et clles périront infailliblement l'hyver suivant."

"Si, pour éviter ce malheur, il entreprend de les nourrir, il sera donc dans la nécessité de leur rendre le miel qu'il leur a pris, et dont il croyoit faire son profit. S'il ne leur cn remet pas à temps, ou en suffisante quantité; si les abeilles, qui ne vivent pas seulement de miel, mais encore de matière à cire, comme cela est plus probable, il se trouve dans l'impuissance de leur en donner; ou enfin si ces ruches ainsi nourries, échappées à la faim, périssent par les pillages qu'occasionnent fréquemment ces nourritures artificielles, où en sera logé le paurre proprićtaire?"

"Je sais que nos auteurs, prétendant domer. des remèdes à tous ces maux, accumulent règles sur règles, soit pour le temps de tailler 
SUR les abeilles. Liv. I. Chap. XIII. 437 les fortes ruches ( sur quoi il n'y a encore rien de certain, les sentimens étant partagés ) soit sur la manière de nourrir les foibles, et qu'enfin ils recommandent de mettre force barricades à l'entrée de ces dernières, pour en éviter les pillages. Mais comment faire entrer dans l'esprit d'un paysan, la plupart du temps grossier, tout ce code de lois, qui forme à peine quelque chose de net pour une personnne attentive et réfléchissante?"

"Voilà eil peu de mots le détail de ce qui a été imaginé, pour, dit-on, ôter facilement aux mouches leurs provisions sans les faire périr. Il est visible que ces différentes méthodes ne parent pas aux deux principaux inconvéniens qu'il est question d'éviter : l'un, d'occasionner la perte entière des ruches pendant l'hiver, si on leur retire beaucoup de provision; l'autre, de ne procurer aucune abondance au public, et presque point de profit au propriétaire, si on leur en retire peu.» 


\section{H A P I T R E X I V.}

Aulres molifs en faveurdu même systême; fondés sur ce que ce sysiême est le plus avanungeux pour la prospérité des abeilles.

«Comme je crois avoir suffisamment prouvé, et dans la préface de cet ouvragre, et clans l’addition ( 1 ) qui précède ces réflexions, que la méthode de ceux-ci est effectivement la plus avantageuse pour la récolte du miel et de la cire, je vais maintenant faire voir qu'clle est de même beaucoup plus favorable à la multiplication des abeilles. Cette assertion paroîtra sans doute au premier abord un paradoxe; mais jespère que la preuve que j'en vais donner, ne la fera pas toujours regarder comme telle."

"On ne peut disconvenir que la multiplication des ruches ne dépende absolument de leur force, et de leur embonpoint : que lorqu'clles sont privées de cet avantage, ou elles n'essaiment

(I) C'est ce que j'ai rapporté dans le chapitre précédent. 
sur les abeilles. Liv. I. Chap. XIV. 439 point, ou elles donnent des essaims très-foibles, qui sont plusieurs années à prendre leur croissance, s'ils ne périssent en chemin. »

* Or il est certain que le dégraissage des ruches ne peut se faire sans altérer considérable. ment leur santé, et qu'il les réduit à un état de foiblesse qui leur fait retenir à la maison les essaims qu'elles enverroient dehors, si elles étoient suffisamment fortes. Tout cet ouvrage prouve, en effet, que ce que Columelle et ceux qui l'ont pris pour guide attribuent aux maladies, ausquelles ils disent que les abeilles sont souvent sujettes, ne doit l'ĉtre qu'à ces dégraissages, et aux nourritures qu'ils mettent dans lobligation de leur fournir pendant l'hiver, lesquelles n'étant pas de leur goût, ne peuvent leur tenir lieu de celles qu'elles amassent ellesmêmes. "

- Done l'usage de dégraisser les ruches ćtant visiblement un principe certain et perpétuel de foiblesse pour elles, doit, par une conséquence nécessaire, être regardé conme un trèsgrand obstacle à leur multiplication. Je crois quoon auroit de la peine à répondre à ce syllogisme."

"Les epéculateurs d'aujourd'hui, qui s'éE. e iv 
puisent à chercher de nouvclles constructions de 1uches propresà chátrer plus facilenent les abcilles, semblent donc vouloir nous ramener à la méthode des anciens. Mais si leurs nouvclles inventions, loin de parer aux inconvéniens des anciennes, les angmentent, par la facilité quelies doment de pouvoir afluiblir à son aise les mouches, et avec moins de danger d'en être vexé, elles sont plus préjudiciables qu'utiles, et il est à désirer que les gens de campagnne ne les adoptent pas. Heureusement leur cherté est seule cápable de les en détourner. »

"Les inconvénicns qui naisscnt du dégraissage des ruches, ne se trouvent point dans celles que l'on exempte de cette opération. Parmi ces dernic̀res, celles que l'on déponilie qui sont toujours les plus anciennes, sont pourvues d'une quantité de micl capable de contenter une personne raisonnable. On y recueille toute la cire qu'il est possible de tirer des ruches que l'on gararde dix ans : ce n'est qu'après en avoir en nombre d'essaims plus que suffisans, pour les remplacer, quon les sacrifie à l'intérét particulier, et à la consommation publique. C'est ainsi que l'on tue un bouf, après en aroir tiré pendant un temps Ies services qu'il étoit ravable de rendre.» 
Sur les abeilles. Liv. I. Chap. XIV. 4tr "Quant aux jeunes ruches destinées à constituer les fonds des propriétaires, si l'on a eu soin de les gouverner comme nous l'avons enscignné, loin de s'afloiblir, elles se fortifient dans le court espace de quatoize à quinze mois, au point de pouroir être elles-mêmes récoltées au bout de ce temps, après avoir pourvu à leur prospérité. Elles ne souffient pas de la rigueur de l'hiver qu'elles ont à passer, à cause du grand nombre de mouches dont elles sont composées, de la sauté parfaite dont elles jouissent, et de l'abondance de provisions dont elles sont pourvues pour cette saison. Elle sont hors des atteintes de l'espéce de chenilles dont nous avons parlé, laquelle lcur est si préjudiciable, qu'elle seule est capable de leur faire détester leur habitation, et est cause, comme dit Columelle, qu'une ruche, quelque soin qu'orı en ait, ne dure guères plus de dix ans. On ne les voit guères en proye aux maladies qui affligent celles quion dégraisse, lesquelles, jo le répète, ne sont autre chose qu'un état de langueur ct d'infirmité auquel ce dégraissaģe les réduit. Enfin les essaims quielles laissent pour leur succéder, sont d'une force et d'une vigueur proportionnécs anx'leurs." 
L'augmentation du fond des ruches d'un proprićtaire ne dépead donc que de lui. (Quiil détruise chaque année deux ou trois anciennes ruches de moins qu'il n'aura recueilli de forts essaims, son fond se trouvera augrmenté tous les ans d'un pareil nombre de vassaux. Cette méthode partiquée par un grand nombre de propriétaires par-tout lc royaume, formera en peu d'années une augmentation considérable de ruches. "

"Dans les annóes où le propriétaire dépouillera peu, parce qu'elles n'auront pas été absolument favorables au jet, il sera dédommagé de la modicité de sa récolte par l'augonentation sur le prix du miel et de la cire que leur rareté occasionnera : de sorte que son jrofit sera ; à peu de chose près, le même dans une année médiocre que dans une bonne.

" Il cn seroit autrement si cette disctte lui étoit personnellc, ou pour avoir mal gouverné ses abeilles, ou pour les avoir laissé dégénérer, en les gardant trop long-tempss daus le même lien, comme il arrive aux semences, on enfin pour avoir roulu en tirer un profit cxcessi?, ou cn en filisant périr un trop gensad nom!)re. Daus ces cas, comme i! ne pouroti impriter son mal- 
sur les abeilles. Liv. I. Chap. XIV $44^{3}$ heur quà lui-même, le remède sera de profiter deses fautes pour n'y plus retomber. »

On voit que nons avons rapporté exactenient tous les raisonnemens de M. La Grenée en faveur de son sytême. Il ne me reprochera certainement pas d'avoir rien omis de tout ce qui peut favoriser son opinion. Si je n'ai pas cité ce qu’il dit sur le même sujet, page I 46 , c'est parce que sans rien présenter de nouveau, il ne fait que confirmer ce qu'il avoit dit dans sa préface sur l'avantage que son systême produit aux proprićtaires. J'ajouterai seulement ici que l'auteur dit, au chapitre 10, par. 6, page 1 37.

"Mais si l'on demande, comment font donc " les habitans de certaines provinces du royaume "qui ont pour systême de n'en jamais faire "périr : Les uns parce que c'est la coutume; " les autres parce quils croiroient bonnement " offenser Dieu? Je répons que ce que les "hommes ont la cupidité de ne point faire, la "Providence ménagèe de ses dons le fáit pour « cux. "

"Il est fort ordinaire dans ces pays de voir " des milliers de ruches périr tous les ans; ce "qui arrive principalement, parce que les ha«bitans se contentant de tailler ou travase! 
"tontes les fortes, et les réduisant par-là au " même état que les foibles, la disctte et les " pillages réciprogues qu'occasionnent les nour"ritures artificiclles, qu'on est obligé de leur "fournir, les ćclaircissent au point que tel pro"priétair e qui pendant l'été s'est vu une centaine "de ruches, est tont stupélait après l'hyver "de ne s'en plus trouver qu'une quarantaine, " et quelquefois moins; et cela après avoir eu " le chagrin de lemrendre pendant l'hyver une "bonne partie du micl quil leur a retiré, et "s'ètre donné des peines infinies pendant cette "saison, dans l'espérance de les réchapper. Si " au lieu de cela, il se füt conduit, comme je "le lui conseille, il auroit fait son profit du "miel que ses morches ont consommé avant * de périr; il aurnit eu ses peines de reste, " ainsi que le déplaisir de n’avoir pas réussi "dans sa folle entreprise. Moins riche cu idées "il l'cût été davantağe en effet."

Après aroir exposé le sentiment de M. La Grenée sur cette question, je vais dire ce que jen pense. 


\section{H A P I T R E VII.}

\section{Sur la question et les difficultés dc M. La Grenée.}

A prés tout ce que nous avons vu vers la fin du chapitre précédent, il sembleroit que partout où l'on pratique l'usage que M. Laa Grenée conclamne, de tailler les ruches sans détruire les abeilles, l'espèce de ces insectes devroit avoir disparu totalement; qu'elle devroit du moins y avoir diminué au point d'être à la veille de sa destruction, et qu'au contraire par-tout ou sa méthode est suivie, comme elle l'est en effet dans certaines provinces de France; d'Italie, et même de Turquie, l'espèce des abeilles devroit se multiplier exclusivement; c'est cependant ce qu'on ne voit ni en France ni dans le reste de l'Europe. Par-tout la culture des abeilles languit, quelque systême qu'on suive dans lcur gourernement: soit qu'on les taille ou non, les longs hivers les emportent, ce sont les plus fortes ruches et les mieux peuplées qui succombent 
446 TRAITÉ COMPLET

le plus souvent. (I) J'en appele à lá bonne foi de M. La Grenée lui-même qui convient avoir perdu, vers la fin de certains hivers rigoureux, des ruches auxquelles il n'avoit pas même touché, pour en retirer le miel ou la cire.

M. Ducarne dit avoir essuyé les mêmes accidens, et qu'il a perdu, dans un hiver 30 ou 40 de ses meilleures ruches sur 80 qu'il psssédoit. Je pourrois nommer plusieurs personnes qui se plaignent des mêmes pertes, entr'autres un amateur à Viroflay, qui s'est amusé à rassembler un certain nombre de ruches, et qui n'en a jamais enlevé le miel, dans la vue de les multiplier avec plus de rapidité. Malgré tout ses soins et ses dépenses pendant 7 à 8 ans, il s'est trouvé réduit l'année dernière à une seule ruche.

Il faut donc convenir que dans les systêmes de tous les auteurs, on n'apprend rien de certain à cet égard: il y a un vice qui ne dépend ni de ce qu'on taille les ruches ni de ce quion ne les châtre pas, mais qui porte sur la façon de les gouverner et sur les influences du climats,

(I) On peut voir au chapitre 9, la raison pour laquelle les. ruches les plus peuplées sont souvent plus exposées que les foibles. 
SUR les abeilles. Liv. I. Chap. XV. 447 et il faut s'attacher à le corriger. Il faut doncen revenir à ce que nous avons dit sur la clécadence de la culture des abeilles, sur leur mortalité, et sur la manière d'y remédier'; c'est-à-dire , qu'il faut trouver une méthode de les gouverner pen. dant l'hiver sûre, simple et facile, et alors nous verrons disparoîtrela grande mortalité des ruches même dans les temps les plus rigoureux.

Une preuve que ce désordre dépend de la façon de les gouverner, c'est que dans l'Archipel on taille toujours les abeilles, et qu'elles n'en sont pas moins en bon état. Il est de notoriété publique dans l'Archipel, et particulièrement dans l'île de Syra, qu'on ne recueille le miel qu'en faisant la récolte des ruches, et que rien ne pourroit déterminer les habitans de nos îles à faire périr leurs abeilles. Cependant malgré cet usage constant et général, elles passent fort bien l'hiver; leur travail commence à son terme, elles fournissent de très-bons essaims, et elles subsistent de ro à 20 ans et quelquefois davantage; ce sont des faits incontestables.

Ce que M. La Grenée avance contre l'usage de vendanger les ruches sans faire périr les abèilles, quelque fondé qu'il soit, sur la méthode de les tailler et de lesgourerner en France, 
ne peut pas détruire notre manière de les soigner et de les vendanger, sous un climat tel que le nôtre.

Mais pour la manière de les gouverner dans ce pays-ci, javoue ingénuement que tout ce qu'il dit en faveur de son opinion contre la pratique contraire, est raisonnable et bien fondé. Voici ce que je dois observer après lui.

La destruction des abeilles, volontaire ou forcée, contribuc plas que tout le reste à réduire la culture des abeilles à un état de langueur fort opposé au point de perfection dont elle seroit susceptible en France et ailleurs. Mais enfin, comme l'observe l'auteur cité, à quoi serviroient au propriétaire et au public tant de recherches et d'expériences pour retirer Ie miel et la cire, sans faire mourir les abeilles, si malgré ces précautions, une partie des ruches ainsi récoltées périt presque aussitôt après l'opération, et si l'autre partie échappée à tant de dangers, périt aussi dans l'hiver faute de vivres?

Nous avous déjà fait remarquer la destruction que ce défaut de vivres opere dans les ruches, même dans les mieux garnies, et auxquelles on n’a point touché pour en récolter le miel 
SUR les abeilles. Lit. I. Chap. XV. $4+9$ miel. Combien ne seroit-elle pas plus considérable dans celles qui auroient été taillées et auxquelles on auroit ôté la moitié de leurs provisions, et dont cependant la population qui nauroit pas soufiert par la récolte, doit être supposée égrale à celle des ruclies restées intactes?

Si j’arois des abeilles à gouverner en France, ct que je fusse astreint à suivre la méillode ordinaire des cultirateurs, tant pour la conformation desruchesque pour la manière de les soigner; au lieu de les tailler, et de me voir privé à la fin de l'hiver de mes abeilles sans presque aucun profit, je préférerois, comme M. Lagrenée, d'en faire périr tous les ans une partie, afin de mapproprier leur dépouille; mais je me garderois bien de me servir de la méthode de tous ces auteurs, qui conseillent de les tailler, et de les exposer ainsi à tous les dangers de cette opération, et à une perte presque certaine, pendant la rigueur de l'hiver. Dés quïl faudroit en faire le sacrifice, jaimerois mieux en faire pénir une partie pour cn tirer tout le profit possible, que de les voir mourir de faim sans preqque aucune espèce d'avantage.

Ce n'est donc point par l'étalage d'une compassion affectée pour lès abeilles, qu'on doit chercher Tome $I$.

$F f$ 
450 TRAITEE COMPLET

à combattre le systême de M. Lagrenée. Il faut lui opposer des faits qui soient fondés sur une pratique solide, constante, et exempte des inconvéniens et des abus qu'il blâme avec tant de force dans les moyens inventés par des auteurs modernes, et employés pour enlever la provision des abeilles sans les faire périr. Il faut lui opposer, avec une manière facile de tailler les ruches, celle de les préserver de la disette et de la mort pendant l'hiver; ce que l'on n'a pas fait jusqu’à présent.

Mais puisqu'il déclare, "que s’il adopte sa * manière de récolter, ce n'est pas parce qu'il « est prévenu contre les raisons qui pourroient "la lui faire rejetter, puisque lui-même, vers * la fin de sa préface, laisse à chacun la liberté " de ses sentimens et de ses pratiques, non" seulement sans blâmer, mais louant au con* traire les efforts de ceux qui en cherchent de " meilleures; car,ajoute-t-il,je ne pense pas qu'on " ait tont trouvé sur cet article; " puisque $M$. Lagrenée, dis-je, pense ainsi, je ne dloute pas qu’il ne fût le premier à renoncer à son systême, si on lui en faisoit connoître un autre qui, en rendant un profit raisonnable aux propriétaires, conserveroit en même temps les ruches pour les années suivantes. 
Sur les abeilles. Liv. I. Chap. XV. 45i C'est ce que je me flatte d'exécuter dans le cours de cet ouvrage, et j’en appellerai à M. Lagrenée lui-même sur la valeur et la force de mes moyens; en attendant, je vais répondre à ses difficultés.

De quoi est-il finalement question entre ceux. qui agitent cette matière? (c'est la demande de M.Lagrenée.) Je réponds qu'elle consisteà trouver ; $1^{\circ}$. un moyen sûr et constant de multiplier en France les abeilles et le nombre de leurs cultivateurs; $2^{\circ}$. de procurer au royaume cette abondance de cire et de miel dont son heureuse si tuation le rend suscepible, et dont il a besoin ; $3^{\circ}$. de rendre la culture des abeilles constamment avantageuse à l'état et aux propriétaires: c'est le vou du public, et ce doit être le but des travaux de tous les auteurs. Ces trois objets sont tellement liés les uns aux autres, qu'ils ne peuvent pas être séparés.

Quant à la multiplication de l'espèce, il cst clair que sa destruction volontaire ou forcée s'y oppose entièrement. Si on la joint au mauvais gouvernement des abeilles, comme nous l'avons dit ailleurs, et comme M. Lagrenée le prouve dans ses deux chapitres précédens, ce sera la principale cause qui empêchera de s'appliquer géné

Ff ij 
ralement à cette culture; et en n'y.remédiant pas, le royaume manquera toujours de la quantité de miel et de cire nécessaire.

La méthode de récolter les ruches, sans faire périr les abeilles, est donc préférable à celle de les faire mourir pour retirer leur miel. Cette vérité sera bien plus sensible, si l'on réfléchit qu'il n'y a pas en France. assez de cultivateurs et de proprićtaires pour introduire dans le royaume l'abondance désirée, ni le nombre de ruches que chaque cultivateur pourroit entretenir suivant ses facultés. Mais ce préalable une fois rempli, qu'on détruise, si l'on veut, les ruches surnuméraires (ou celles qu'on ne pourroit conserver faute d'emplacement ou d'autres moyens) pour en retirer un plus grand profit; ce n'est qu'alors que la destruction peut être permise.

Notre auteur répondra peut-être, que ce systême seroit bon si les ruches récoltées pouvoient seconserveren bon état, et échapper à l'âpreté et à la longueur des hivers, puisqu'il n'est pas douteux, qu'en y ajoutant les nouveaux essaims qu'elles fourniroient tous les ans, cela feroit renaître l'abondance de la cire et du miel; mais, comme on a prouvé que pendant ou après cette 
SUR les abeilles. Liv. I. Chap. XV. 453 récolte, une grande partie des abeilles périt, et presque toujours dans l'hiver, il est clair que cette manière de récolter est préjudiciable aux abeilles, et désavantageuse aux propriétaires.

A cela je réponds que de la manière dont nous taillons nos ruches, il arrive tres-rarement qu'elles périssent par une suite de la récolte. Pendant plus de 15 ans que j’ai gouverné des abeilles et que je les ai toujours récoltées, il ne m'est jamais arrivé de perdre une seule ruche dans cette circonstance. Au reste, si l'on suit la méthode que jindiquerai pour la récolte du miel en France, je soutiens qu'il n'arrivera jamais aucun nial aux abeilles. On pourra voir ce que je dirai dans tout mon septième livre sur cette récolte.

Quant aux ruches qui périssent dans l'hiver', cela arrive non-seulement aux ruches récoltées, mais à celles qui ne le sont pas; et j'en ai expliqué moi-même les raisons ci-dessus au $9^{e}$. chapitre. Il faut donc absolument chercher le moyen d'empêcher que lesprovisions ne manquent et aux ruches taillées et à celles qui ne le sont pas; ce malheur vient toujours de leur trop grande consommation pendant l'hiver : pour l'empêcher, j’ai déja proposé plus haut un moyen 
454 TRAITÉ C OMPLET efficale, me réservant de donner, dans le livre suivant, la manière de tenir les ruches renfermées pendant les mauvais temps de l'hiver, pour mettre les abeilles en état d'économiser leurs provisions.

On comprendra aisément que si cela peut réussir, comme je n'en doute pas, notre méthode de récolter les ruches, sans faire périr les abeilles, est in finiment préférable à celle de M. Lagrenée, tant pour lintérêt des propriétaires, et pour procurer l'abondance de la cire au royaume, que pour la prospérité de l'espèce des abeilles.

Son calcul sur la différence du profit que feroient deux propriétaires qui suivroient les deux méthodes opposées, tombe de lui-même, après ce que nous venons d'observer : car en supposant que d'après ses principes, il ait retiré de la dépouille d'une ruche $40 \mathrm{l}$. de miel,tandis que d'après les miens, je n’en aurois retiré que $\mathbf{r} 5$, dix même si l'on veut ; sije gouverne bien ma ruche, et que je la conserve pendant ro ans, elle me rendra la même quantité de miel tous les ans. Il y a plus, la ruche détruite n'aura donné à M. Lagrenée qu'un seul essaim, au lieu que celle que j’aurai conservée pendant 10 ans, m’en aura douné au moins 5 , sans parler même de 
sur les abeilles. Liv. I. Chap. XV. 455 ceux que ces cinq auront pu produire. Je demande à présent qui de nous aura plus de profit de sa 'ruche, au bout de ces dix années, ou de M. Lagrenée qui a détruit la sienne, après en avoir retiré quarante livres de miel et un essaim, oude moi qui, après avoir conservé la mienne pendant dix ans, ai eu, outre cent livres de miel, cinq essaims, et le produit de ces cinq es. saims ? qui de M. Lagrenće ou de moi aura contribuć à procurer une plus grande abondance de cire et de miel à l'état? Que M. Lagrenće juge lui-même.

On dira peut-être que la grande question est de savoir si une ruche ainsi récoltée peut se conserver pendant dix ans. J'assure positivement qu'il en est ainsi chez nous, ct que cela doit 'être en France, si l'on suit notre méthode. Je me flatte de le prouver en parlant du temps que vivent les abeilles.

Après ce que je viens de dire, il n'est pas difficile de répondre aux autres raisonnemẹs de M. Lagrenée, sur l'arantage de sa méthode pour la prospérité des abeilles, et pour leur multiplication. Il est certain, que la conservation de ces insectes dépend de leur force et de leur vigueur. Mais je nie que ces deux qua- 
456 TRAITE C O AI PET

lités. soient des attributs propres uniquement aux ruches non récoltées, et que celles qui sont vendangées ne puissent en jouir.

Cette force des ruches ne peut s'entendre que du nombre d'abeilles qui les composent, ou de la quantité de leurs rayons et de leur provision. Quant aı nombre d'abeilles, il doit être égal dans les ruches récoltées et dans celles qui ne le sont pas; puisque la méthoke dont nous nous servons pour récolter nos ruches à Syra, et que j’ai perfectionnée, est si facilc et si commode, que jamais les abeilles ne peuvent en souffir. Nous le prouverons en temps et lieu.

Au reste, pour ce qui est du grand nombre d'ab illes dans l'automne, temps auquel on les récolte, M. Lagrenée ne doit pas s'en inquiéter, lui qui prétend daus sa préface, que cette grande population leur est nuisible pondant cette saison et dans l'hiver.

Si, par la force d'une ruche, on entend la quantité de micl et de molividhe, je dirai quil suflit qu'elle ait assez de provisions pour passer 'hiver, et quil lui en reste au printemps, pour que les abeilles puissent commencer à former leurs premieres couvées: elle donnera 
Sur les abeilles. Liv. I. Chap. XV. 457 de bons essains, et aussi hâtifs que celle où l'on n'aura pas touché.

Au surplus, si quelquefois une ruche taillée avoit besoin d'un supplément de miel, nous avons dans notre méthode la plus grande facilité de le ini procurer, sans craindre aucun danger du pillage, comme on peut le voir au 6e. livre. Enfin relativement à la quantité des rayons, je dirại que sept à huit gâteaux de nos ruches sont suffisans pour contenir les provisions de nos insectes, et pour lesabriter dans l'hiver; un trop grand nombre de rayons de cire seroit préjudiciable aux ruches, sur-tout s’ils étoient vides de miel. Si l'on veat qu'elles se conservent, il faut proportionner les rayons à leur population, pour que les abeilles puissent les couvrir, et les nettoyer au moins au printemps. Sans cette précaution, les vers attaquent souvent les rayons découverts, et sans un prompt secours les ruches sont perdues. C'est une des raisons pour lesquelles elles sont si souvent en France la proie de cette vermine : à peine peuventelles s'en préserver plus de trois ou quatre ans, parce que plusicurs propriétaires laissent leurs ruches pleines de rayons entiers sans y toucher, 
et que les abeilles ne sont pas en assez grand nombre pour les couvrir et pour les nettoyer. Les rayons ainsi découverts sont facilement attaqués par les vers, ou infectés par la moisissure : je parle de ceux qui sont vides; car, je le répète, les vers ne se mettent pas dansceux qui sont bien garnis de miel.

Ainsi le systême de M. Lagrenée de ne point toucher aux ruches, de ne rien enlever de leurs provisions, est fort désavantageux à l'espèce.

Et qu'on ne dise pas que celles que l'on conserve sont à l'abri des vers, étant composées presque toutes de nouveaux essaims qui pendant deux ou trois ans ne peuvent pas les craindre.Comment nos auteurs auroient-ils pu avancer une telle erreur, après ce qui arrive souvent à Syra? Les ruches de nos jeunes essaims, dans les mauvaises années, sont souvent attaquées par de fausses teignes, lorsque les propriétaires nont pas eu soin de les nettoyer; c'est ce que j’ai observé moi-même à Viroflay dans un essaim d'environ deux mois, dont un rayon s'étoit détaché en remuant la ruche, à la suite. d'un coup violent qu'elle avoit essuyé : iy comptai plus de 15 vers qui sortoient du fond des collules à moitié remplies de molividhe; et on remar- 
sur les abeilles. Liv. I. Chap. XV. 459 quera que l'essaim étoit en bon état et couvroit tous les rayons. Qu'on juge de ce qui doit en résulter, quand les choses ne sont pas ainsi.

Enfin, ce raisonnement que M. Lagrenée croyoit victorieux et sans replique, est, comme on voit, peu concluant et sans force.

Les abeilles d'une ruzhe taillée sont pour l'ordinaire plus empressées de construire de nouveaux rayons, que celles d'une ruche dont on n'a retiré ni rayon, ni provision; et l'avantage en est aussi grand pour le propriétaire que pour le public.

Je n’en répéterai pas moins ici ce quejai dit ailleurs, que quoique les observations de M. Lagrenée ne s'accordent pas avec la méthode que je proposerai pour récolter les ruches, elles sont cependant très-bonnes pour combattre l'ancienne maniềre qui est entièrement défectucuse; et il faut conclure, tant de ses raisonnemens que des 'miẹns, qu’il est de nécessité indispensable de réformer en France l'économie des abcilles, si l'on veut qu'elles y produisent tous les avantages que l'on peut en attendre. Pour cela, il faut y admettre généralement la méthorle que je propose dans ce traité, sur la structure et la disposition de nos ruchers, et sur la ma- 
460 TR R T T E C O M P E T nière de les récolter et de les gouverner pendant l'hiver.

En attendant, je pense que jusqu’à ce que le royanme soit à peu près pourvu du nombre de propriétaires et de ruches qu’il peut soutenir, le gouvernement doit prendre des moyens pour empêcher les cultivateurs de détruire les abeilles en leur enlevant le fruit de leurs travaux. La nécessité seule devroit autoriser les propriétaires à détruire le surplus des ruches qu'ils ne peuvent entretenir ; encore cet excédent d'abeilles pourroit-il être cmployé souvent à garnir quelque ruche trop foible.

C'étoit le but des vues sages de l'Empereur, quand il promettoit un florin de récompense pour chaque ruche qu'on cultiveroit dans ses états, pourvu qu'on observât de n'en faire la revue qu'au printemps; car ce Prince savoit bien que l'on n'auroit pas intérêt de les détruire dans cette saison.

FIN DU TOME PREMIER。 


\section{T A B L E}

D E S C H A P I T R E S

Contenus dans le Tome premier.

PRECIS HISTORIQUE ET ÉCONOMIQUE SUR L'îLE DE S Y RA.

Avant-propos........................ I

Cн A P I'T R I. 'Tableau rapide des íles de l'Archipel, d'après l'histoire des Dncs de Naxie.. 3

C н A p I-T r E II. De l'état arcien de l'jle de Syra.. 29

CH A P IT R E III. De l'ancien monmment astronomique deSyra, et du philosople Phérécyde... 36

Ch A р I т R E I V. État actuel de l'ile de Syra... 57

Cha pitre $V$. Du gourernement temporel et spirituel de Syra...................... $7^{2}$

Сиа p itre VI. Protection du Roi de France enrers les habitans de lîle de Syra, relativement à leur religion ; et reconnoissance de ceux-ci envers les Francuis................. 90

Chл p т те V'If. Du earactère et du génie des Syriutes................... I 13

Ch а р I t r e VIU. Courte réfutation de M. Paw, 
sur ce qu'il dit des Grees modernes... . . . Page 13.5

C R A P I'T R IX. De la Langue des Syriotes et des autres Grecs muderues............ I 54

Cна p I т R E X. De quelques procédés particuliers, relatifs à l'agriculture et à l'économie domestique. I 95

C h A P I T R E XI. Méthode de caprifier le figuier, usitée à Syra et dans toute la Grèce, depuis les temps les plus reculés.............. $23 x$

CH а р I т и е X11. Moyen simple et facile dont se servent les habitans de l'ile de Syra, pour guérir la jannisse................... 268

\section{TRAITÉ COMPLETSUR LES ABEILLES.}

\section{I V R E P R E M I E R,}

Coutenarit tout ce qui regarde la culture des abcilles en général.

Cir a p I T R E I. Des pays les plıs propres à élever des abeilles, de la quantité de ruches que chaque pays pcut nourrir, et de la situation la plus avantagcuse quil comvient de leur clomer. . . . . . . . 28 9 CHAPITRE II. Des arbres et des plantes dont les fleurs servent i lit nomrriture des abeilles... 305

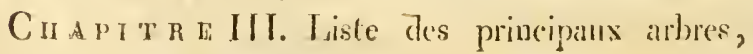


arbustes et fleurs qui servent à la nourriture des abeilles.................... Page 320

C н A P I T R I V. Catalogue des autres plantes et arbrisseaux, dont les fleurs sont utiles aux abeitles .................... 33 .

C н A P I т R E V. Des plantes qui dounent an miel une qualitè nuisible aux abeilles et aux hommes ....................... 346

ChApItre VI. Des arantages qu'un État peut retirer de la culture des abeilles. . . . . . . . 355

Ch A p i t r e VII. Causes de la décadence de la culture des abeilles en France.......... 376

Cha p it Re VIII. Moyen efficace de relever la culture des abeilles............... 384

Ch A p I t r e IX. Causes de la mortalité des abeilles... . . . . . . . . . . . . . . . . . . 394

Ch A p I t R E X. Manière d'ériter les mortalités dont on vient de parler. . . . . . . . . . . 403

Ch $А$ р т т E XI. De la manière de gourerner les abeilles pendant l'hiser, de les empêcher de consommer leurs provisious, et d'éviter leur ciestruction. 409

C н A P I T R E XII. Est-il plus arantageux de faire périr les abeilles pour retirer des ruches tout le miel et la cire, ou de se servir, pour y parrenir, de tout autre moyen? Détail de ce qu'on a fait jusqu'à présent................ 42 
462 Ta bLE Des C II a titres. Cil apitre XIII. Motifs en fareur dusystême de faire périr les abcilles ponr ret irer leurs provisions, fonde's sur ce que cette pratigue est de la plus grande utilite aux pooprietaires.... Pa Pagc 428

Chapitre XIV. Antres motifs en faveur du même systême, fundès sur ce que cce systême est. le plus avantagens pour la prospérit $\dot{c}$ des abcilles. 4.38 Сан р гт в е XV. Sur la question et. les difficultés de M. La Grence............... . 445 FI N I) E I. A T \& I L E. 




(2) ing

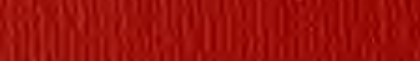

W19.

(1)

MIM

MIII)

s.1.

g||

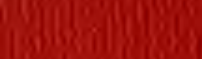

Will

Sol|

\section{(1)}

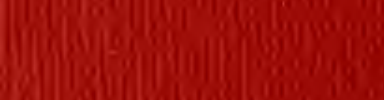

form

(1)

is

Ingin

60)

130

6)

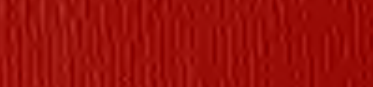

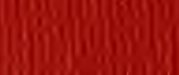

somong

yong

Wy

aly

(1)

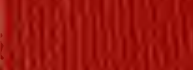

(1)

IX)

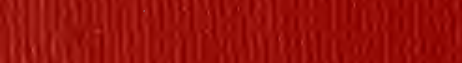

(1)

11)

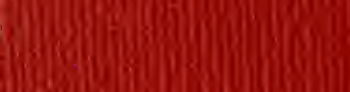

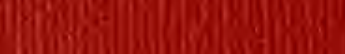

